\title{
Imaging blood-brain barrier function in aging
}

Citation for published version (APA):

Verheggen, I. C. M. (2021). Imaging blood-brain barrier function in aging. [Doctoral Thesis, Maastricht University]. Maastricht University. https://doi.org/10.26481/dis.20210601iv

Document status and date:

Published: 01/01/2021

DOI:

10.26481/dis.20210601iv

Document Version:

Publisher's PDF, also known as Version of record

\section{Please check the document version of this publication:}

- A submitted manuscript is the version of the article upon submission and before peer-review. There can be important differences between the submitted version and the official published version of record.

People interested in the research are advised to contact the author for the final version of the publication, or visit the DOI to the publisher's website.

- The final author version and the galley proof are versions of the publication after peer review.

- The final published version features the final layout of the paper including the volume, issue and page numbers.

Link to publication

\footnotetext{
General rights rights.

- You may freely distribute the URL identifying the publication in the public portal. please follow below link for the End User Agreement:

www.umlib.nl/taverne-license

Take down policy

If you believe that this document breaches copyright please contact us at:

repository@maastrichtuniversity.nl

providing details and we will investigate your claim.
}

Copyright and moral rights for the publications made accessible in the public portal are retained by the authors and/or other copyright owners and it is a condition of accessing publications that users recognise and abide by the legal requirements associated with these

- Users may download and print one copy of any publication from the public portal for the purpose of private study or research.

- You may not further distribute the material or use it for any profit-making activity or commercial gain

If the publication is distributed under the terms of Article $25 \mathrm{fa}$ of the Dutch Copyright Act, indicated by the "Taverne" license above, 


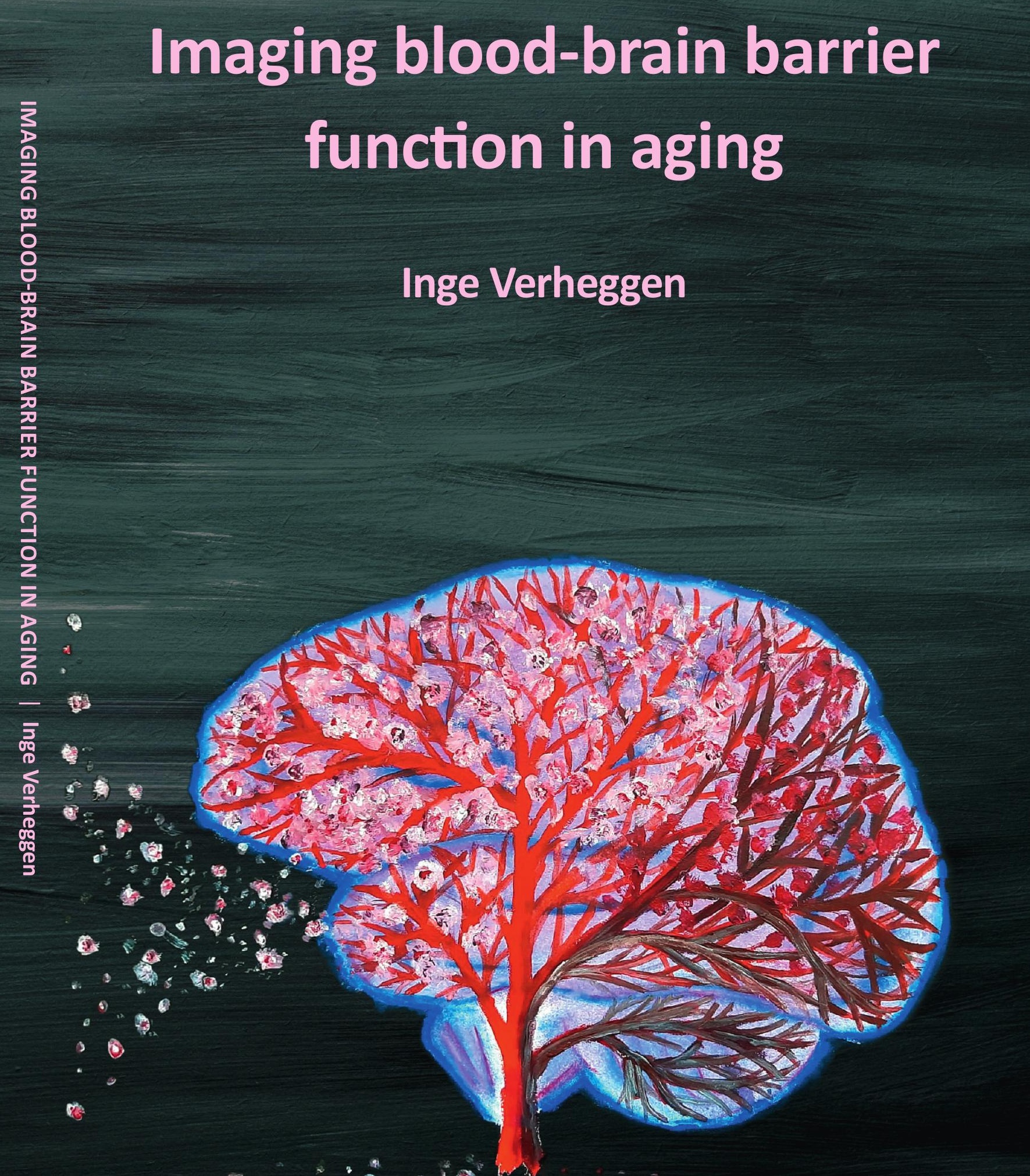


Imaging blood-brain barrier function in aging 
(C) 2021 Inge Catharina Maria Verheggen - All rights reserved.

No part of this publication may be reproduced, stored in a retrieval system or transmitted in any form or by any means, electronic, mechanical, photocopying, recording or otherwise, without the prior permission of the author.

Print: Ridderprint | www.ridderprint.nl

Cover art by Iris van Sambeek

Printing of this thesis was financially supported by Maastricht University. 


\title{
Imaging blood-brain barrier function in aging
}

\author{
PROEFSCHRIFT
}

ter verkrijging van de graad van doctor aan de Universiteit Maastricht,

op gezag van de Rector Magnificus, Prof. Dr. Rianne M. Letschert

volgens het besluit van het College van Decanen,

in het openbaar te verdedigen

op dinsdag 1 juni 2021 om 16.00 uur door

Inge Catharina Maria Verheggen

Geboren 23 september 1991 te Weert 


\section{Promotors:}

Prof. Dr. Ir. Walter H. Backes

Prof. Dr. Frans R.J. Verhey

\section{Co-promotor:}

Dr. Martin P.J. van Boxtel

\section{Assessment committee:}

Prof. Dr. Werner H. Mess, Maastricht University Medical Center (chair)

Prof. Dr. Jeroen Hendrikse, University Medical Center Utrecht

Prof. Dr. Arjan Blokland, Maastricht University

Dr. Jurgen A.H.R. Claassen, Radboud University

Dr. Heidi Jacobs, Maastricht University and Harvard Medical School

This work was supported by the Nederlandse Organisatie voor Wetenschappelijk Onderzoek (NWO) [Personal Grant I.C.M.V. number 406-15-031]. 


\section{Table of contents}

Page

\section{Chapter 1:}

Introduction

\section{Chapter 2:}

Increase in blood-brain barrier leakage in healthy, older adults

Original article, GeroScience (2020)

\section{Chapter 3:}

Imaging the role of blood-brain barrier disruption in normal cognitive aging

Original article, GeroScience (2020)

\section{Chapter 4:}

Permeability of the windows of the brain: Feasibility of dynamic contrast-enhanced MRI of the circumventricular organs

Original article, Fluids and Barriers of the CNS (2020)

\section{Chapter 5:}

Interaction between blood-brain barrier and glymphatic system in solute clearance

Review article, Neuroscience and Biobehavioral Reviews (2018)

\section{Chapter 6:}

The application of contrast-enhanced MRI in the assessment of

blood-cerebrospinal fluid barrier integrity

Review article, Neuroscience and Biobehavioral Reviews (2021)

\section{Chapter 7:}

Discussion

Summary 

Chapter 1

\section{Introduction}

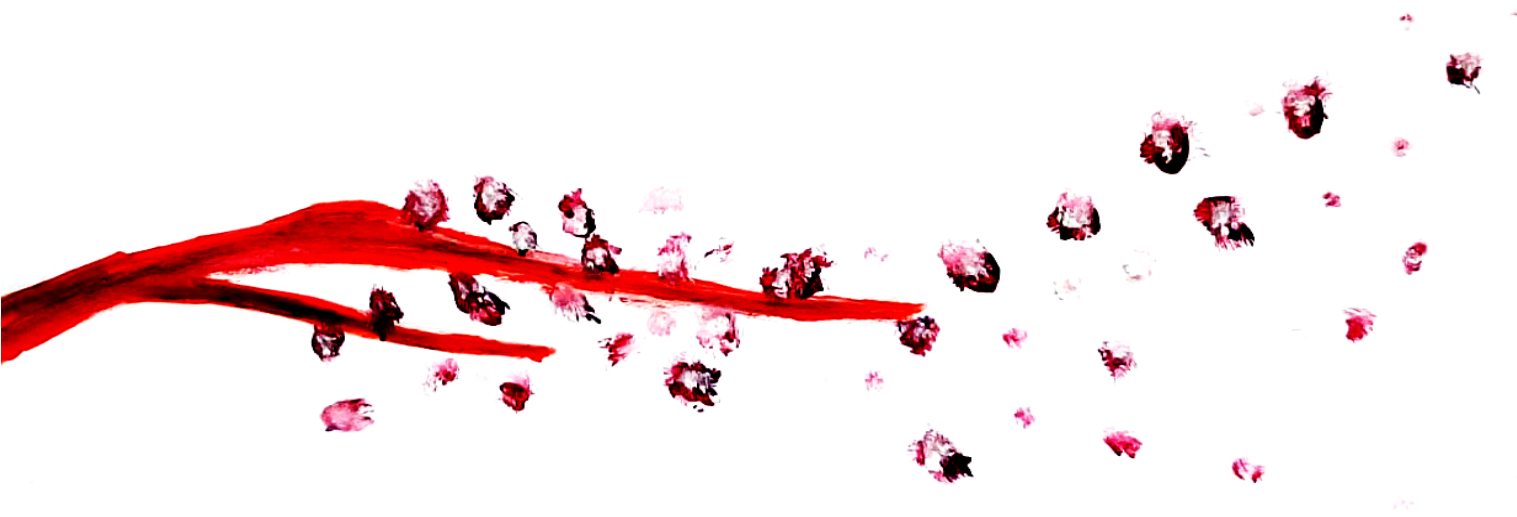




\subsection{Successful aging and the brain}

Population demographics are shifting towards a larger proportion of older individuals, as declining birth rates and increasing life expectancy are resulting in rapidly aging populations all over the world (1). In developed European countries, populations have been aging for decades, and Europe is expected to remain the oldest region in the years to come. For a continent in which more than a quarter of the population will be aged 65 or over by 2050 , strategies to promote successful aging are of great importance. Studies investigating what factors determine successful aging have become an increasingly important scientific objective. Successful aging can be defined as the avoidance of disease and disability, maintenance of high physical and cognitive functioning, and sustained engagement in social and productive activities (2). The maintenance of cognitive functioning is thus an important component of successful aging. An aging population is increasingly confronted with age-related neurobiological changes, and such changes in brain structure and function are often associated with changes in cognition $(3,4)$. Studies into the causes and prevention of age-related disorders are essential, as the prevalence of these disorders increases when more people reach older ages. Moreover, people who age without any overt pathological condition may still experience some degree of agerelated cognitive decline, though this decline shows large interindividual differences (5). Some people age without considerable cognitive setback, while others show substantial cognitive decline $(4,6)$. Investigating what neurobiological changes lead to age-related cognitive decline and hinder successful aging has become an urgent research topic $(4,7)$. Factors that may reduce chances of successful aging, for instance genetic factors such as carrying the $\varepsilon 4$ allelle of the apolipoprotein E gene (APOE4) (8) or lifestyle factors such as physical inactivity or obesity (9), often seem to relate to vascular health.

Age has a large impact on the vascular system and is a major risk factor for neurovascular damage $(10,11)$. Neurovascular disease is a highly prevalent age-related condition (12). Neurovascular dysfunction could be a promising condition to study in relation to age-related cognitive decline $(13,14)$.

\subsection{The role of the blood-brain barrier}

At the level of the cerebral microvasculature, specialized cells work together in a neurovascular unit (NVU) to maintain cerebral blood flow (CBF), oxygen delivery and energy supply (15). The NVU consists of vascular cells (i.e. endothelial cells), glial cells 
(i.e. astrocytes) and neurons $(15,16)$. Interaction between vascular cells and neurons in the NVU creates hemodynamic coupling, providing an appropriate CBF response to an increase in neuronal activation $(17,18)$. The endothelial cells are connected by tight junctions to form a strong barrier, located between the blood and the brain parenchyma $(15,19)$. At the parenchymal side, endothelial cells are supported by astrocytes and pericytes $(20,21)$. This collection of cells and structures in the cerebrovascular wall separating the blood plasma from the brain tissue constitutes the blood-brain barrier (BBB) (Figure 1.1).

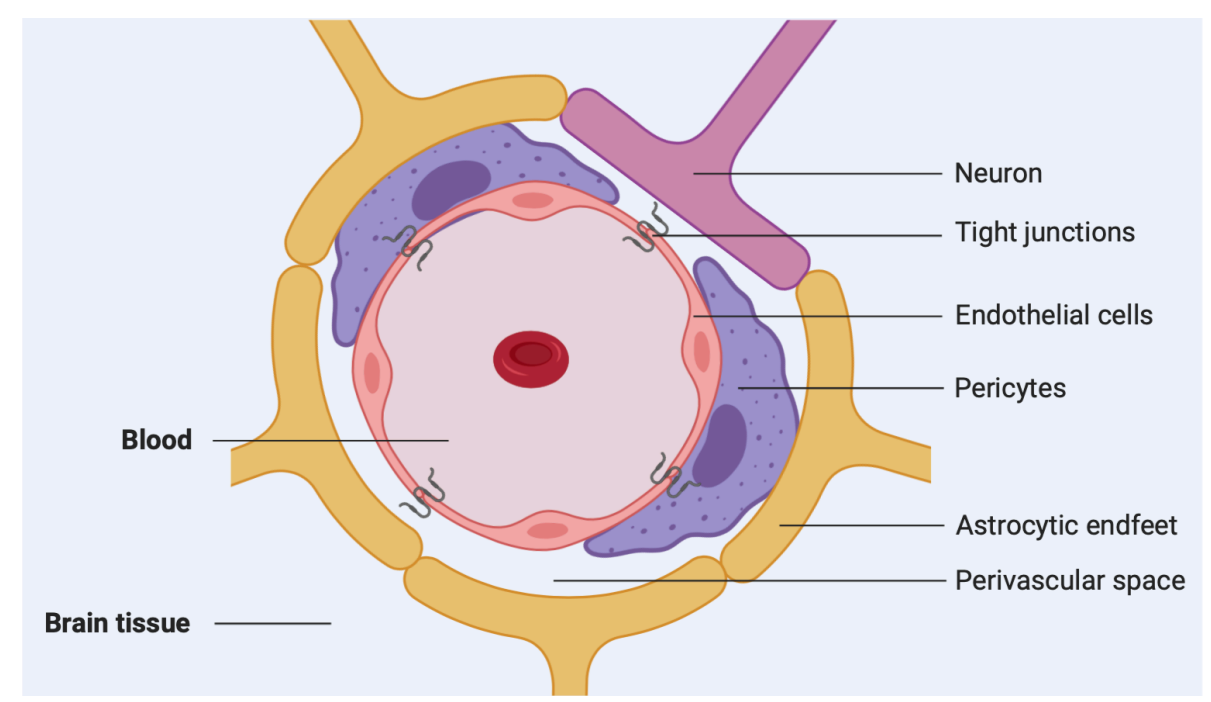

Figure 1.1 Cross-section of a capillary schematically depicting the structure of the blood-brain barrier.

The BBB regulates the delivery of energy metabolites and nutrients to the neurons, while preventing neurotoxins from entering the brain. Small molecules, such as oxygen and carbon dioxide, can freely diffuse across the BBB, which is essential for brain metabolism and $\mathrm{pH}$ regulation $(22,23)$. As for the rest, the endothelial cells contain specialized transport systems, using active efflux via efflux pumps, carrier-mediated transport and receptor-mediated transport, to enable energy metabolites and nutrients to enter the brain tissue and reach the neurons $(22,24)$. Potentially neurotoxic waste products (e.g. the amyloid- $\beta$ protein $(A \beta)$ ) can be removed from the brain either through the glymphatic system or across the BBB. The glymphatic system is a waste clearance mechanism using the cerebral microcirculation to clear solutes from the interstitial space, by exchanging interstitial fluid (ISF) with cerebrospinal fluid (CSF). Interstitial solutes can also be transported across the BBB by specific transport proteins, and removed via the blood 
Chapter 1

stream. The BBB thereby protects the brain from ion fluctuations that occur in the blood after a meal or exercise and helps ensuring homeostasis in the central nervous system (CNS), meaning that the precise chemical balance in the neuronal tissue essential for adequate neuronal and synaptic functioning is maintained $(25,26)$.

\subsection{Neurovascular dysfunction and BBB disruption}

Homeostasis is namely dependent on the dynamics between the neurofluids: a collective name for the blood, CSF and ISF (27). Several pathological conditions have been associated with a disturbance in neurofluid dynamics, such as sleep disorders (28), Alzheimer's disease (AD) (29), Parkinson's disease (30), traumatic brain injury (31) and stroke (32), which can be grouped under the term CNS interstitial fluidopathies (33). One way in which neurofluid dynamics can be disrupted is by neurovascular dysfunction.

Age impacts the neurovascular system and neurovascular alterations occur over time $(10,11)$. Neurovascular alterations can involve uncoupling of the NVU, which can lead to a reduction and dysregulation of the $\mathrm{CBF}$ and disruption of the $\mathrm{BBB}(34,35)$. Neurovascular alterations can also involve endothelial dysfunction, such as impaired endothelial cell proliferation and migration, as studies have found more endothelial dysfunction in older individuals (36-38). Aging has been associated with deficiency of the nicotinamide adenine dinucleotide $\left(\mathrm{NAD}^{+}\right)$enzyme precursor, which is involved in cellular proliferation and function (39-41). Age-related $\mathrm{NAD}^{+}$depletion has been associated with endothelial dysfunction, and restoring the $\mathrm{NAD}^{+}$levels appears to protect the integrity of the neurovascular system (41-45). Thus, age-related $\mathrm{NAD}^{+}$deficiency can lead to neurovascular dysfunction over time and may contribute to BBB disruption.

Neurovascular dysfunction has been suggested to initiate the pathological cascade that can eventually lead to neurodegeneration $(15,25,46)$. Both reduced CBF and BBB disruption impair the supply of oxygen and nutrients to the neurons. As a result, hypoxia and inflammation may occur and trigger subsequent pathological processes, which make the brain vulnerable to neuronal dysfunction, and eventually neurodegeneration $(11,47$, 48). Moreover, BBB disruption reduces waste clearance from the brain and could lead to accumulation of toxic waste products, such as $A \beta$, which is strongly associated with AD (49). In turn, $A \beta$ can further impair neurovascular functioning, including CBF regulation and BBB integrity. These neurovascular alterations occur even before the formation of $A \beta$ plaques, suggesting these are early events in the pathological cascade (50-52), which 
is supported by several imaging studies demonstrating early BBB disruption in the development of AD (53-56). Studies have even shown that carrying APOE4, indicating genetic susceptiblility to $\mathrm{AD}(8)$, is already associated with accelerated BBB disruption (57-59).

BBB disruption is thought to be one of the underlying mechanisms of cerebral small vessel disease (cSVD) $(60,61)$, which refers to pathology in the small capillaries of the brain $(62,63)$. cSVD is characterized by neuroimaging markers such as white matter hyperintensities (WMHs), lacunar infarction, enlarged perivascular spaces and microbleeds (62). AD, on the other hand, is usually characterized by Alzheimer pathology, such as $A \beta$ accumulation, tau tangle formation and cortical volume loss (64). In reality, the processes underlying vascular dementia and AD can occur in parallel and the expressed pathology is often a combination between markers of cSVD and Alzheimer pathology $(65,66)$. As mentioned before, BBB disruption can lead to $A \beta$ accumulation due to reduced clearance, and the neurotoxic A $\beta$ can further impair the BBB (49). Thus, BBB disruption does not only have a major role in vascular causes of dementia, but can also promote Alzheimer pathology, and it might be a common trigger for age-related brain pathology (67).

\subsection{BBB disruption and cognitive functioning}

Besides possibly being an early event in the pathological cascade and a common trigger for age-related brain pathology $(54,56)$, BBB disruption has also been associated with global cognitive functioning, as leakage rates in individuals with early AD and healthy age-matched controls correlated significantly with cognitive functioning (e.g. in studies using the Mini-Mental State Examination (MMSE) as outcome (68)) (56). Moreover, individuals with early cognitive dysfunction, not accounted for by a neurological disorder, demonstrated increased BBB disruption, independent of the amount of $A \beta$ or tau pathology (69). BBB disruption seems to be already present in those who experience age-related cognitive decline, even without having a neurological disorder. A recent study demonstrated that BBB disruption is not only more prominent in APOE4 carriers, but this effect was stronger in cognitively impaired individuals, which suggests that BBB disruption is involved in APOE4-related cognitive decline (70). BBB disruption might be a contributing mechanism to cognitive aging. 
Chapter 1

\subsection{Measuring BBB permeability}

Interest in BBB disruption is increasing due to recent discoveries about its role in brain pathology, but also due to recent advances made in the development of techniques to measure BBB leakage $(47,71)$. Interest has shifted from measuring high leakage rates in diseases with large BBB damage, such as tumors and multiple sclerosis (MS), to measuring with lower leakage rates in conditions with more subtle BBB disruption, such as cerebral small vessel disease and dementia (47).

First, post-mortem studies demonstrated significant BBB disruption in dementia patients (72-75), but these studies could only shed light on the end-stage of the disorder. For a long time, the albumin blood/CSF ratio has been used as a biomarker for BBB permeability (76-78), with the BBB being more permeable if more albumin passes from the blood to the CSF. However, the albumin ratio cannot localize BBB leakage, and with subtle BBB disruption, albumin only slowly moves into the CSF, needing a relatively long time to detect a subtle leak, which has led to the need for more efficient methods to quantify BBB permeability (47). Positron emission tomography (PET) has been used (79, 80 ), but this technique requires radioactive tracers and is methodologically complex due to the manufacturing and half-life of the tracers.

One of the most promising techniques is dynamic contrast-enhanced magnetic resonance imaging (DCE MRI), which measures contrast agent transfer from the blood plasma to the brain tissue over time, and allows the detection of subtle leakage values (81). A gadolinium-based contrast agent is intravenously injected during MRI scanning, to quantify and localize the spread of the contrast from the blood plasma to the brain tissue, which is dependent on the extent of $\mathrm{BBB}$ disruption. A dual-time resolution protocol can ideally be applied, in which scans with a high temporal resolution are used during contrast agent injection to capture the rapid spread over the vasculature, and scans with a lower temporal resolution are used during the slower leakage phase (82, 83). Using this technique, a significantly larger BBB leakage rate was found in individuals with mild cognitive impairment (MCI) (84), often considered the transition state to AD, or with cSVD (85), the leading cause of vascular dementia (62). This technique was also able to detect the significantly higher BBB leakage rates in older adults with early cognitive dysfunction compared to individuals showing no cognitive impairment, even in the absence of any neurological or psychiatric condition (69). 


\subsection{Study objectives}

Thus, previous studies suggest that BBB disruption might trigger age-related brain pathology and might be a contributing mechanism to cognitive aging $(61,69,84,85)$. However, DCE MRI studies investigating how BBB disruption is associated with age or age-related cognitive decline in healthy individuals are lacking. A better understanding of how BBB disruption is involved in the normal aging process could benefit the interpretation of BBB leakage observed in pathological processes.

Therefore, for this thesis, we conducted a study to investigate whether BBB leakage can be related to age and cognitive decline in normal aging individuals. This study was conducted as part of the Maastricht Aging Study (MAAS) (86), a long-running study into cognitive aging in which a large group of participants was cognitively followed over a long period of time, using medical history and data from cognitive testing. From this study, we selected cognitively and neurologically healthy individuals to participate in our study. We hypothesized that older age would be significantly associated with higher BBB leakage rates. As BBB disruption might be a trigger for further age-related brain pathology, we expected the brain regions involved in higher-order cognitive functions, that are most sensitive to age-related deterioration, to be especially vulnerable to agerelated BBB breakdown. Also, we hypothesized that more BBB leakage would be significantly associated with a greater decline in cognitive functioning, before the manifestation of other age-related brain abnormalities, suggesting that BBB disruption is an early event in cognitive aging.

\subsection{Contents of the thesis}

First, we investigated whether older age is associated with more BBB disruption, even in cognitively and neurologically healthy individuals, and whether this association was stronger for brain regions sensitive to age-related impairment (Chapter 2). Moreover, we investigated whether this BBB disruption was paired with more normal age-related cognitive decline (Chapter 3). We also conducted a feasibility study aiming to validate our technique by measuring contrast transfer in structures known to not have a BBB, namely the circumventricular organs (CVOs) (Chapter 4). To further explore the waste clearance role of the $\mathrm{BBB}$, we conducted a review attempting to resolve how BBB transport relates to glymphatic function when it comes to waste clearance, and how these clearance mechanisms interact (Chapter 5). Finally, we conducted a review on the blood-CSF 


\section{Chapter 1}

barrier (BCSFB), as the role of the BCSFB in maintaining homeostasis in the CNS seems to be more important than previously thought (87), and we considered recent developments made in assessing BCSFB permeability using contrast-enhanced MRI (Chapter 6). These chapters will be followed by a general discussion, further highlighting the most important findings, providing considerations for future research and concluding remarks.

\subsection{References}

1. He W, Goodkind D, Kowal PR. An aging world: 2015. 2016.

2. Rowe JW, Kahn RL. Successful aging. The gerontologist. 1997;37(4):433-40.

3. Glisky EL. Changes in cognitive function in human aging. Brain aging: models, methods, and mechanisms. 2007:3-20.

4. Rapp PR, Amaral DG. Individual differences in the cognitive and neurobiological consequences of normal aging. Trends in neurosciences. 1992;15(9):340-5.

5. Woodruff-Pak DS. The neuropsychology of aging: Blackwell Publishing; 1997.

6. Cabeza R, Albert M, Belleville S, Craik FI, Duarte A, Grady CL, et al. Maintenance, reserve and compensation: the cognitive neuroscience of healthy ageing. Nature Reviews Neuroscience. 2018;19(11):701-10.

7. Bishop NA, Lu T, Yankner BA. Neural mechanisms of ageing and cognitive decline. Nature. 2010;464(7288):529-35.

8. Yamazaki Y, Zhao N, Caulfield TR, Liu C-C, Bu G. Apolipoprotein E and Alzheimer disease: pathobiology and targeting strategies. Nature Reviews Neurology. 2019;15(9):501-18.

9. Deckers K, van Boxtel MP, Schiepers OJ, de Vugt M, Muñoz Sánchez JL, Anstey KJ, et al. Target risk factors for dementia prevention: a systematic review and Delphi consensus study on the evidence from observational studies. Int J Geriatr Psychiatry. 2015;30(3):234-46.

10. Camici GG, Liberale L. Aging: the next cardiovascular disease? European Heart Journal. 2017;38(21):1621-3.

11. Yang T, Sun Y, Lu Z, Leak RK, Zhang F. The impact of cerebrovascular aging on vascular cognitive impairment and dementia. Ageing research reviews. 2017;34:15-29.

12. Choi JY, Morris JC, Hsu CY. Aging and cerebrovascular disease. Neurologic clinics. 1998;16(3):687711.

13. Farkas E, Luiten PG. Cerebral microvascular pathology in aging and Alzheimer's disease. Progress in neurobiology. 2001;64(6):575-611.

14. Norton E, Bridges L, Kenyon L, Esiri M, Bennett D, Hainsworth A. Cell Senescence and Cerebral Small Vessel Disease in the Brains of People Aged 80 Years and Older. Journal of Neuropathology and Experimental Neurology. 2019;78.

15. Zlokovic BV. Neurovascular pathways to neurodegeneration in Alzheimer\&\#39;s disease and other disorders. Nature Reviews Neuroscience. 2011;12:723. 
16. Wilhelm I, Nyúl-Tóth Á, Suciu M, Hermenean A, Krizbai IA. Heterogeneity of the blood-brain barrier. Tissue Barriers. 2016;4(1):e1143544.

17. Buxton RB, Uludağ K, Dubowitz DJ, Liu TT. Modelling the hemodynamic response to brain activation. Neuroimage. 2004;23:S220-S33.

18. Guo S, Lo EH. Dysfunctional cell-cell signaling in the neurovascular unit as a paradigm for central nervous system disease. Stroke. 2009;40(3_suppl_1):S4-S7.

19. Hawkins RA, Peterson DR, Vina JR. The complementary membranes forming the blood-brain barrier. IUBMB life. 2002;54(3):101-7.

20. Prat A, Biernacki K, Wosik K, Antel JP. Glial cell influence on the human blood-brain barrier. Glia. 2001;36(2):145-55.

21. Balabanov R, Dore-Duffy P. Role of the CNS microvascular pericyte in the blood-brain barrier. Journal of neuroscience research. 1998;53(6):637-44.

22. Zhao Z, Nelson AR, Betsholtz C, Zlokovic BV. Establishment and dysfunction of the blood-brain barrier. Cell. 2015;163(5):1064-78.

23. Pardridge WM. Blood-brain barrier endogenous transporters as therapeutic targets: a new model for small molecule CNS drug discovery. Expert opinion on therapeutic targets. 2015;19(8):1059-72.

24. Montagne A, Zhao Z, Zlokovic BV. Alzheimer's disease: A matter of blood-brain barrier dysfunction? Journal of Experimental Medicine. 2017;214(11):3151-69.

25. Zlokovic BV. The blood-brain barrier in health and chronic neurodegenerative disorders. Neuron. 2008;57(2):178-201.

26. Wardlaw J, Sandercock P, Dennis M, Starr J. Is breakdown of the blood-brain barrier responsible for lacunar stroke, leukoaraiosis, and dementia? Stroke. 2003;34(3):806-12.

27. Agarwal N, Contarino C, Toro EF. Neurofluids: A holistic approach to their physiology, interactive dynamics and clinical implications for neurological diseases. Veins and Lymphatics. 2019;8(3).

28. DiNuzzo M, Nedergaard M. Brain energetics during the sleep-wake cycle. Current opinion in neurobiology. 2017;47:65-72.

29. Schubert JJ, Veronese M, Marchitelli L, Bodini B, Tonietto M, Stankoff B, et al. Dynamic 11C-PiB PET shows cerebrospinal fluid flow alterations in Alzheimer disease and multiple sclerosis. Journal of Nuclear Medicine. 2019;60(10):1452-60.

30. Sundaram S, Hughes RL, Peterson E, Müller-Oehring EM, Bronte-Stewart HM, Poston KL, et al. Establishing a framework for neuropathological correlates and glymphatic system functioning in Parkinson's disease. Neuroscience \& Biobehavioral Reviews. 2019;103:305-15.

31. Christensen J, Wright DK, Yamakawa GR, Shultz SR, Mychasiuk R. Repetitive mild traumatic brain injury alters glymphatic clearance rates in limbic structures of adolescent female rats. Scientific reports. 2020;10(1):1-9.

32. Gaberel T, Gakuba C, Goulay R, De Lizarrondo SM, Hanouz J-L, Emery E, et al. Impaired Glymphatic Perfusion After Strokes Revealed by Contrast-Enhanced MRI. Stroke. 2014:STROKEAHA. 114.006617.

33. Taoka T, Naganawa S. Imaging for central nervous system (CNS) interstitial fluidopathy: disorders with impaired interstitial fluid dynamics. Japanese Journal of Radiology. 2020. 


\section{Chapter 1}

34. Kisler K, Nelson AR, Montagne A, Zlokovic BV. Cerebral blood flow regulation and neurovascular dysfunction in Alzheimer disease. Nature Reviews Neuroscience. 2017;18:419.

35. Iadecola C. The Neurovascular Unit Coming of Age: A Journey through Neurovascular Coupling in Health and Disease. Neuron. 2017;96(1):17-42.

36. Ungvari Z, Tarantini S, Donato AJ, Galvan V, Csiszar A. Mechanisms of vascular aging. Circulation research. 2018;123(7):849-67.

37. Murugesan N, Demarest TG, Madri JA, Pachter JS. Brain regional angiogenic potential at the neurovascular unit during normal aging. Neurobiology of aging. 2012;33(5):1004. e1-. e16.

38. Ungvari Z, Tucsek Z, Sosnowska D, Toth P, Gautam T, Podlutsky A, et al. Aging-induced dysregulation of dicer1-dependent microRNA expression impairs angiogenic capacity of rat cerebromicrovascular endothelial cells. Journals of Gerontology Series A: Biomedical Sciences and Medical Sciences. 2013;68(8):877-91.

39. Yoshino J, Baur JA, Imai S-i. NAD+ intermediates: the biology and therapeutic potential of NMN and NR. Cell metabolism. 2018;27(3):513-28.

40. Gomes AP, Price NL, Ling AJ, Moslehi JJ, Montgomery MK, Rajman L, et al. Declining NAD+ induces a pseudohypoxic state disrupting nuclear-mitochondrial communication during aging. Cell. 2013;155(7):1624-38.

41. Csiszar A, Tarantini S, Yabluchanskiy A, Balasubramanian P, Kiss T, Farkas E, et al. Role of endothelial NAD+ deficiency in age-related vascular dysfunction. American Journal of Physiology-Heart and Circulatory Physiology. 2019;316(6):H1253-H66.

42. Kiss T, Balasubramanian P, Valcarcel-Ares MN, Tarantini S, Yabluchanskiy A, Csipo T, et al. Nicotinamide mononucleotide (NMN) treatment attenuates oxidative stress and rescues angiogenic capacity in aged cerebromicrovascular endothelial cells: a potential mechanism for the prevention of vascular cognitive impairment. GeroScience. 2019;41(5):619-30.

43. Tarantini S, Yabluchanskiy A, Csipo T, Fulop G, Kiss T, Balasubramanian P, et al. Treatment with the poly (ADP-ribose) polymerase inhibitor PJ-34 improves cerebromicrovascular endothelial function, neurovascular coupling responses and cognitive performance in aged mice, supporting the NAD+ depletion hypothesis of neurovascular aging. GeroScience. 2019;41(5):533-42.

44. Mills KF, Yoshida S, Stein LR, Grozio A, Kubota S, Sasaki Y, et al. Long-term administration of nicotinamide mononucleotide mitigates age-associated physiological decline in mice. Cell metabolism. 2016;24(6):795-806.

45. Zhang H, Ryu D, Wu Y, Gariani K, Wang X, Luan P, et al. NAD+ repletion improves mitochondrial and stem cell function and enhances life span in mice. Science. 2016;352(6292):1436-43.

46. Zlokovic BV. Neurovascular mechanisms of Alzheimer's neurodegeneration. Trends in Neurosciences. 2005;28(4):202-8.

47. Raja R, Rosenberg GA, Caprihan A. MRI measurements of Blood-Brain Barrier function in dementia: A review of recent studies. Neuropharmacology. 2018;134:259-71.

48. Fernando MS, Simpson JE, Matthews F, Brayne C, Lewis CE, Barber R, et al. White matter lesions in an unselected cohort of the elderly. Stroke. 2006;37(6):1391-8. 
49. Burgmans S, van de Haar HJ, Verhey FR, Backes WH. Amyloid- $\beta$ interacts with blood-brain barrier function in dementia: a systematic review. Journal of Alzheimer's Disease. 2013;35(4):859-73.

50. Iadecola C, Zhang F, Niwa K, Eckman C, Turner SK, Fischer E, et al. SOD1 rescues cerebral endothelial dysfunction in mice overexpressing amyloid precursor protein. Nature Neuroscience. $1999 ; 2: 157$.

51. Niwa K, Kazama K, Younkin L, Younkin SG, Carlson GA, Iadecola C. Cerebrovascular autoregulation is profoundly impaired in mice overexpressing amyloid precursor protein. American Journal of PhysiologyHeart and Circulatory Physiology. 2002;283(1):H315-H23.

52. Niwa K, Kazama K, Younkin SG, Carlson GA, Iadecola C. Alterations in Cerebral Blood Flow and Glucose Utilization in Mice Overexpressing the Amyloid Precursor Protein. Neurobiology of Disease. 2002;9(1):61-8.

53. Iturria-Medina Y, Sotero RC, Toussaint PJ, Mateos-Pérez JM, Evans AC, The Alzheimer's Disease Neuroimaging I, et al. Early role of vascular dysregulation on late-onset Alzheimer's disease based on multifactorial data-driven analysis. Nature Communications. 2016;7:11934.

54. Montagne A, Barnes SR, Sweeney MD, Halliday MR, Sagare AP, Zhao Z, et al. Blood-brain barrier breakdown in the aging human hippocampus. Neuron. 2015;85(2):296-302.

55. Montagne A, Nation DA, Pa J, Sweeney MD, Toga AW, Zlokovic BV. Brain imaging of neurovascular dysfunction in Alzheimer's disease. Acta neuropathologica. 2016;131(5):687-707.

56. van de Haar HJ, Burgmans S, Jansen JF, van Osch MJ, van Buchem MA, Muller M, et al. Blood-brain barrier leakage in patients with early Alzheimer disease. Radiology. 2016;281(2):527-35.

57. Bell RD, Winkler EA, Singh I, Sagare AP, Deane R, Wu Z, et al. Apolipoprotein E controls cerebrovascular integrity via cyclophilin A. Nature. 2012;485(7399):512-6.

58. Salloway S, Gur T, Berzin T, Tavares R, Zipser B, Correia S, et al. Effect of APOE genotype on microvascular basement membrane in Alzheimer's disease. J Neurol Sci. 2002;203-204:183-7.

59. Halliday MR, Rege SV, Ma Q, Zhao Z, Miller CA, Winkler EA, et al. Accelerated pericyte degeneration and blood-brain barrier breakdown in apolipoprotein E4 carriers with Alzheimer's disease. J Cereb Blood Flow Metab. 2016;36(1):216-27.

60. Wardlaw J. Blood-brain barrier and cerebral small vessel disease. Journal of the neurological sciences. 2010;299(1):66-71.

61. Farrall AJ, Wardlaw JM. Blood-brain barrier: ageing and microvascular disease-systematic review and meta-analysis. Neurobiology of aging. 2009;30(3):337-52.

62. Pantoni L. Cerebral small vessel disease: from pathogenesis and clinical characteristics to therapeutic challenges. The Lancet Neurology. 2010;9(7):689-701.

63. Wardlaw JM, Smith EE, Biessels GJ, Cordonnier C, Fazekas F, Frayne R, et al. Neuroimaging standards for research into small vessel disease and its contribution to ageing and neurodegeneration. The Lancet Neurology. 2013;12(8):822-38.

64. Jack CR, Knopman DS, Jagust WJ, Shaw LM, Aisen PS, Weiner MW, et al. Hypothetical model of dynamic biomarkers of the Alzheimer's pathological cascade. The Lancet Neurology. 2010;9(1):119-28.

65. Schneider JA, Arvanitakis Z, Bang W, Bennett DA. Mixed brain pathologies account for most dementia cases in community-dwelling older persons. Neurology. 2007;69(24):2197-204. 


\section{Chapter 1}

66. Echávarri C, Burgmans S, Caballero MC, García-Bragado F, Verhey FR, Uylings H. Co-occurrence of different pathologies in dementia: implications for dementia diagnosis. Journal of Alzheimer's Disease. 2012;30(4):909-17.

67. Sironi L, Guerrini U, Tremoli E, Miller I, Gelosa P, Lascialfari A, et al. Analysis of pathological events at the onset of brain damage in stroke-prone rats: A proteomics and magnetic resonance imaging approach. Journal of neuroscience research. 2004;78(1):115-22.

68. Folstein MF, Folstein SE, McHugh PR. "Mini-mental state": a practical method for grading the cognitive state of patients for the clinician. Journal of psychiatric research. 1975;12(3):189-98.

69. Nation DA, Sweeney MD, Montagne A, Sagare AP, D’Orazio LM, Pachicano M, et al. Blood-brain barrier breakdown is an early biomarker of human cognitive dysfunction. Nature Medicine. 2019.

70. Montagne A, Nation DA, Sagare AP, Barisano G, Sweeney MD, Chakhoyan A, et al. APOE4 leads to blood-brain barrier dysfunction predicting cognitive decline. Nature. 2020;581(7806):71-6.

71. Larsson HB, Courivaud F, Rostrup E, Hansen AE. Measurement of brain perfusion, blood volume, and blood-brain barrier permeability, using dynamic contrast-enhanced T1-weighted MRI at 3 tesla. Magnetic Resonance in Medicine: An Official Journal of the International Society for Magnetic Resonance in Medicine. 2009;62(5):1270-81.

72. Wisniewski HM, Kozlowski PB. Evidence for Blood-Brain Barrier Changes in Senile Dementia of the Alzheimer Type (SDAT). Annals of the New York Academy of Sciences. 1982;396(1):119-29.

73. Tomimoto H, Akiguchi I, Suenaga T, Nishimura M, Wakita H, Nakamura S, et al. Alterations of the blood-brain barrier and glial cells in white-matter lesions in cerebrovascular and Alzheimer's disease patients. Stroke. 1996;27(11):2069-74.

74. Ujiie M, Dickstein DL, Carlow DA, Jefferies WA. Blood-brain barrier permeability precedes senile plaque formation in an Alzheimer disease model. Microcirculation. 2003;10(6):463-70.

75. Zipser B, Johanson C, Gonzalez L, Berzin T, Tavares R, Hulette C, et al. Microvascular injury and blood-brain barrier leakage in Alzheimer's disease. Neurobiology of aging. 2007;28(7):977-86.

76. Mecocci P, Parnetti L, Reboldi GP, Santucci C, Gaiti A, Ferri C, et al. Blood-brain-barrier in a geriatric population: barrier function in degenerative and vascular dementias. Acta Neurologica Scandinavica. 1991;84(3):210-3.

77. Algotsson A, Winblad B. The integrity of the blood-brain barrier in Alzheimer's disease. Acta Neurologica Scandinavica. 2007;115(6):403-8.

78. Bowman GL, Kaye JA, Quinn JF. Dyslipidemia and blood-brain barrier integrity in Alzheimer's disease. Current gerontology and geriatrics research. 2012;2012.

79. Schlageter NL, Carson RE, Rapoport SI. Examination of Blood - Brain Barrier Permeability in Dementia of the Alzheimer Type with [68Ga]EDTA and Positron Emission Tomography. Journal of Cerebral Blood Flow \& Metabolism. 1987;7(1):1-8.

80. Minoshima S, Giordani B, Berent S, Frey KA, Foster NL, Kuhl DE. Metabolic reduction in the posterior cingulate cortex in very early Alzheimer's disease. Annals of Neurology. 1997;42(1):85-94.

81. van de Haar HJ, Burgmans S, Hofman PA, Verhey FR, Jansen JF, Backes WH. Blood-brain barrier impairment in dementia: current and future in vivo assessments. Neuroscience \& Biobehavioral Reviews. 2014. 


\section{Introduction}

82. Jelescu IO, Leppert IR, Narayanan S, Araújo D, Arnold DL, Pike GB. Dual-temporal resolution dynamic contrast-enhanced MRI protocol for blood-brain barrier permeability measurement in enhancing multiple sclerosis lesions. Journal of Magnetic Resonance Imaging. 2011;33(6):1291-300.

83. van de Haar HJ, Jansen JFA, Jeukens CRLPN, Burgmans S, Buchem MA, Muller M, et al. Subtle bloodbrain barrier leakage rate and spatial extent: Considerations for dynamic contrast-enhanced MRI. Medical Physics. 2017;44(8):4112-25.

84. Wang H, Golob EJ, Su M-Y. Vascular volume and blood-brain barrier permeability measured by dynamic contrast enhanced MRI in hippocampus and cerebellum of patients with MCI and normal controls. Journal of Magnetic Resonance Imaging. 2006;24(3):695-700.

85. Zhang CE, Wong SM, van de Haar HJ, Staals J, Jansen JF, Jeukens CR, et al. Blood-brain barrier leakage is more widespread in patients with cerebral small vessel disease. Neurology. 2017;88(5):426-32. 86. Jolles J, Houx P, Van Boxtel M, Ponds R. Maastricht aging study: Determinants of cognitive aging: Neuropsych Publishers Maastricht; 1995.

87. Ghersi-Egea JF, Strazielle N. Brain drug delivery, drug metabolism, and multidrug resistance at the choroid plexus. Microscopy research and technique. 2001;52(1):83-8. 

Chapter 2

\subsection{Abstract}

\section{Background}

Blood-brain barrier (BBB) breakdown can disrupt nutrient supply and waste removal, which affects neuronal functioning. Currently, dynamic contrast-enhanced (DCE) MRI is the preferred in-vivo method to quantify BBB leakage. Dedicated DCE MRI studies in normal aging individuals are lacking, which could hamper value estimation and interpretation of leakage rate in pathological conditions. Therefore, we applied DCE MRI to investigate the association between BBB disruption and age in a healthy sample.

\section{Methods}

57 cognitively and neurologically healthy, middle-aged to older participants (mean age: 66 years, range: 47 - 91 years) underwent MRI, including DCE MRI with intravenous injection of a gadolinium-based contrast agent. Pharmacokinetic modelling was applied to contrast concentration time-curves to estimate BBB leakage rate in each voxel. Subsequently, leakage rate was calculated in the white and grey matter, and primary (basic sensory and motor functions), secondary (association areas) and tertiary (higherorder cognition) brain regions. A difference in vulnerability to deterioration was expected between these regions, with especially tertiary regions being affected by age.

\section{Results}

Higher BBB leakage rate was significantly associated with older age in the white and grey matter, and also in tertiary, but not in primary or secondary brain regions.

\section{Conclusion}

Even in healthy individuals, BBB disruption was stronger in older persons, which suggests BBB disruption is a normal physiologically aging phenomenon. Age-related increase in BBB disruption occurred especially in brain regions most vulnerable to agerelated deterioration, which may indicate that BBB disruption is an underlying mechanism of normal age-related decline.

Netherlands Trial Register number: NL6358, date of registration: 2017-03-24

\section{Keywords}

Blood-brain barrier, normal aging, dynamic contrast-enhanced MRI, permeability 
Increase in blood-brain barrier leakage in healthy, older adults

\subsection{Introduction}

Currently, an increasing number of people are reaching older ages (1), and studies into the causes and prevention of age-related disorders are becoming increasingly important. However, people who age without any overt pathological condition may still experience some degree of age-related decline (2). This decline shows large interindividual differences (3), while it remains unclear what factors determine whether someone will be strongly affected by age or hardly experience any age-related setback. Cerebral microvascular alterations, occurring over time and contributing to blood-brain barrier (BBB) breakdown, could be a promising topic to study in relation to normal age-related decline $(4,5)$.

In the cerebral microvasculature, the vessel wall of the small capillaries contains endothelial cells connected by tight junctions, forming the basis of the BBB (6). The BBB separates the blood from the brain parenchyma and regulates the delivery of energy metabolites and nutrients to the neurons, while preventing neurotoxins from entering the brain tissue. The endothelium contains specialized transport systems that allow nutrients to move from the blood to the brain, while waste products are removed in the opposite direction $(7,8)$. The BBB thereby protects the brain from concentration fluctuations that occur in the blood stream, which is essential for neuronal and synaptic functioning (9).

Endothelial dysfunction, such as impaired endothelial cell proliferation and migration, is one of the cerebral microvascular alterations occurring over time, as studies have found more endothelial dysfunction in elderly individuals (10-12). Also, aging has been associated with nicotinamide adenine dinucleotide ( $\left.\mathrm{NAD}^{+}\right)$deficiency (13-15). $\mathrm{NAD}^{+}$is a coenzyme, enzyme precursor or substrate involved in cellular proliferation and function, and the regulation of cellular energetics and mitochondrial metabolism $(14,16)$. Age-related $\mathrm{NAD}^{+}$depletion has been associated with endothelial dysfunction, and restoring the $\mathrm{NAD}^{+}$levels appears to protect the integrity of the cerebral microvasculature $(15,17-20)$. Thus, $\mathrm{NAD}^{+}$deficiency affecting endothelial integrity could be an important cerebral microvascular alteration that contributes to BBB disruption over time.

BBB disruption impairs the oxygen and glucose supply, which may produce hypoxia and hypoxia induced inflammation $(21,22)$. Subsequent pathological processes make the brain vulnerable to neuronal dysfunction and could even lead to 
neurodegeneration (23). Moreover, BBB disruption reduces the clearance of interstitial solutes from the brain and could result in accumulation of toxic waste products, such as amyloid- $\beta(A \beta)$ in case of Alzheimer's disease (AD) (24). $A \beta$ was found to further impair neurovascular functioning and the cerebral circulation, even before the actual formation of plaques, suggesting that these neurovascular alterations are an early event in the pathological cascade (25-28). Imaging studies also support that neurovascular dysfunction and BBB disruption occur early in the development of AD (29-32).

A large meta-analysis has suggested that BBB permeability increases already as part of normal aging (33). However, in these studies the blood/CSF albumin ratio was used as biomarker for BBB leakage, which does not differentiate between blood-brain and blood-cerebrospinal fluid leakage and also cannot localize leakage. Nowadays, a more direct method to detect subtle permeability values is dynamic contrast-enhanced (DCE) MRI (21). A gadolinium-based contrast agent is intravenously injected during scanning, to quantify and localize the spread of contrast from the blood plasma to the brain interstitial fluid, which is dependent on the extent of BBB disruption. To distinguish the rapidly circulating component from the slowly extravasating component, dual-time resolution can ideally be applied $(34,35)$. This acquisition technique applies a fast sequence to capture steep signal changes during the initial circulation phase, and a slow sequence during the later leakage phase. Using this technique, significantly larger BBB leakage rate was found in persons with mild cognitive impairment (36), often considered the transition state to $\mathrm{AD}$, and cerebral small vessel disease (37), the leading cause of vascular dementia (38). A recent study even demonstrated that older adults with early cognitive dysfunction had significantly more BBB leakage than individuals showing no cognitive impairment, even in the absence of any neurological or psychiatric condition (39).

These studies suggest that BBB breakdown is part of the normal aging process and occurs even in the absence of neurological disorders. However, DCE MRI studies investigating whether older individuals have stronger BBB disruption, even when these individuals are considered healthy, have not been conducted. The lack of DCE MRI studies in normal aging individuals could hamper the value estimation and interpretation of BBB leakage observed in recent studies on brain pathology. Therefore, we aimed to investigate the association between BBB leakage and age in healthy, middle-aged to older individuals using DCE MRI. 
Higher-order brain regions involved in high-level cognition, such as decision making, planning and related executive functions, are the first brain regions to show agerelated deterioration (40). After the higher-order brain regions, the association areas are most vulnerable to age-related impairment, while areas involved in primary sensory and motor functions tend to remain robust as a function of normal aging (41). Our first hypothesis was that older age would be associated with higher BBB leakage rate throughout the cerebral white and grey matter. Secondly, with BBB disruption possibly being an underlying mechanism for normal age-related deterioration, we hypothesized that the association between BBB leakage and age will be most clearly present in those regions most affected by aging, which are the higher-order brain regions.

\subsection{Methods}

\subsubsection{Participants}

57 participants (mean age: 66 years, age range: 47 - 91 years) were recruited from participants of the Maastricht Aging Study (MAAS) (42), in which 1,823 people were cognitively followed from 1992 until 2005. Only normal aging individuals, considered healthy, were eligible for participation. In this study, 'healthy' was defined as no substantial decrease in global cognition (Mini-Mental State Examination (MMSE; (43)) score $\geq 25$ ), no cognitive impairment due to substance abuse and no great difficulties in performing activities of daily living (Disabilities Assessment of Dementia (DAD; (44)) score $\geq 90 \%$ ), as well as no major neurological conditions (no diagnosis of dementia, prodromal dementia, mild cognitive impairment or any other psychiatric or neurological disorder, and no major structural brain abnormalities, brain surgery or brain trauma as known from the medical history). Furthermore, individuals could only be included when they had no contraindications for MRI or use of gadolinium-based contrast agent (sufficiently functioning kidneys as indicated by an estimated Glomerular Filtration Rate $(\mathrm{eGFR})>30 \mathrm{~mL} / \mathrm{min})$.

\subsection{2. $\quad$ Sample characteristics}

For each participant, age and sex were registered. Experienced neuroradiologists visually rated white matter lesion load according to the Fazekas scale (45) (W.P.) and brain atrophy using the global cortical atrophy (GCA) scale (46) (A.P.), to confirm our sample could be considered neurologically healthy. 
Blood pressure was measured twice and the mean systolic blood pressure was calculated. Weight and height were measured to calculate the body mass index (BMI). Participants were also asked about the occurrence of diabetes and their smoking habits, after which they were classified as diabetic or non-diabetic and smoker or non-smoker. Systolic blood pressure, BMI, diabetes and smoking habits were taken into consideration as potential confounders, as these factors may influence neurovascular health and BBB integrity (47-50).

\subsubsection{MRI acquisition}

Sagittally oriented slices were acquired on a 3-Tesla MRI scanner (Achieva TX, Philips Healthcare, Best, The Netherlands) with a 32-channel head coil. The imaging protocol consisted of 3D T1-weighted inversion recovery fast gradient echo (repetition time (TR)/inversion time (TI)/echo time (TE): 8/800/4 ms; flip angle: $8^{\circ}$; cubic voxel size: 1 $\mathrm{mm}$ ) for anatomical reference, 3D T2-weighted fluid attenuation inversion recovery (FLAIR) (TR/TI/TE: 4800/1650/290 ms; flip angle: 90; cubic voxel size: $1 \mathrm{~mm}$, no slice gap) for the white matter hyperintensity (WMH) volume and dual-time resolution dynamic contrast-enhanced (DCE) MRI for the leakage measurement.

Dual-time DCE MRI consisted of two alternating sequences with a saturation prepulse to provide two time-resolutions: a fast sequence with a dynamic scan interval of $3.2 \mathrm{~s}$ for high temporal resolution to capture the steep signal changes during the initial circulation phase of the contrast agent, and a slow sequence with a dynamic scan interval of $30.5 \mathrm{~s}$ with lower temporal, but higher spatial resolution during the slower leakage phase of the contrast agent (35). The fast sequence consisted of 29 volumes (TR/TE/delay time (TD): 5.3/2.5/120 ms; voxel size: 2 × 2 × $5 \mathrm{~mm}$ ) and the slow sequence consisted of 30 volumes (TR/TE/TD: 5.3/2.5/120 ms; voxel size: 1 x 1 x 2 mm). Before contrast agent administration, scans of both sequences were acquired. Next, during the fast sequence, gadolinium-based contrast agent $(0.1 \mathrm{mmol} / \mathrm{kg}$ gadobutrol, Gadavist $\AA$, Bayer AG, Leverkusen, Germany) was intravenously injected in the antecubital vein (injection rate $3 \mathrm{~mL} / \mathrm{s}, 20 \mathrm{~mL}$ saline flush). Beforehand, T1-mapping was performed to enable conversion of tissue signal intensity to contrast agent concentration (51). 
Increase in blood-brain barrier leakage in healthy, older adults

\subsubsection{Brain segmentation}

WMH volume $\left[\mathrm{cm}^{3}\right]$ was determined by creating WMH masks on the FLAIR images using the in-house developed, semi-automatic segmentation tool GIANT (52). WMH volume was corrected for intracranial volume.

FreeSurfer software (version 6.0.0) was used for automated brain region segmentation (53), combined with a visual check with manual adjustments (I.C.M.V.). From these segmentations, cortical thickness [mm] and hippocampal volume $\left[\mathrm{cm}^{3}\right]$ were obtained. We refer to the brain regions involved in primary sensory and motor functions as primary brain regions, to the association areas as secondary brain regions and to the regions for higher-order cognitive functions as tertiary brain regions. The FreeSurfer software subdivides the cerebral cortex according to the Desikan-Killiany atlas (54). Using this atlas, two brain regions were selected for each of the three classes: for the primary brain regions the precentral and postcentral gyrus, for the secondary brain regions the supramarginal and superior temporal gyrus, and for the tertiary brain regions the orbitofrontal and cingulate cortex (Figure 2.1) $(40,41)$. For our analyses, total white matter, total grey matter (cortical and deep grey matter and hippocampus), and primary, secondary and tertiary brain regions were extracted to create tissue masks for each region of interest (ROI) (53). 


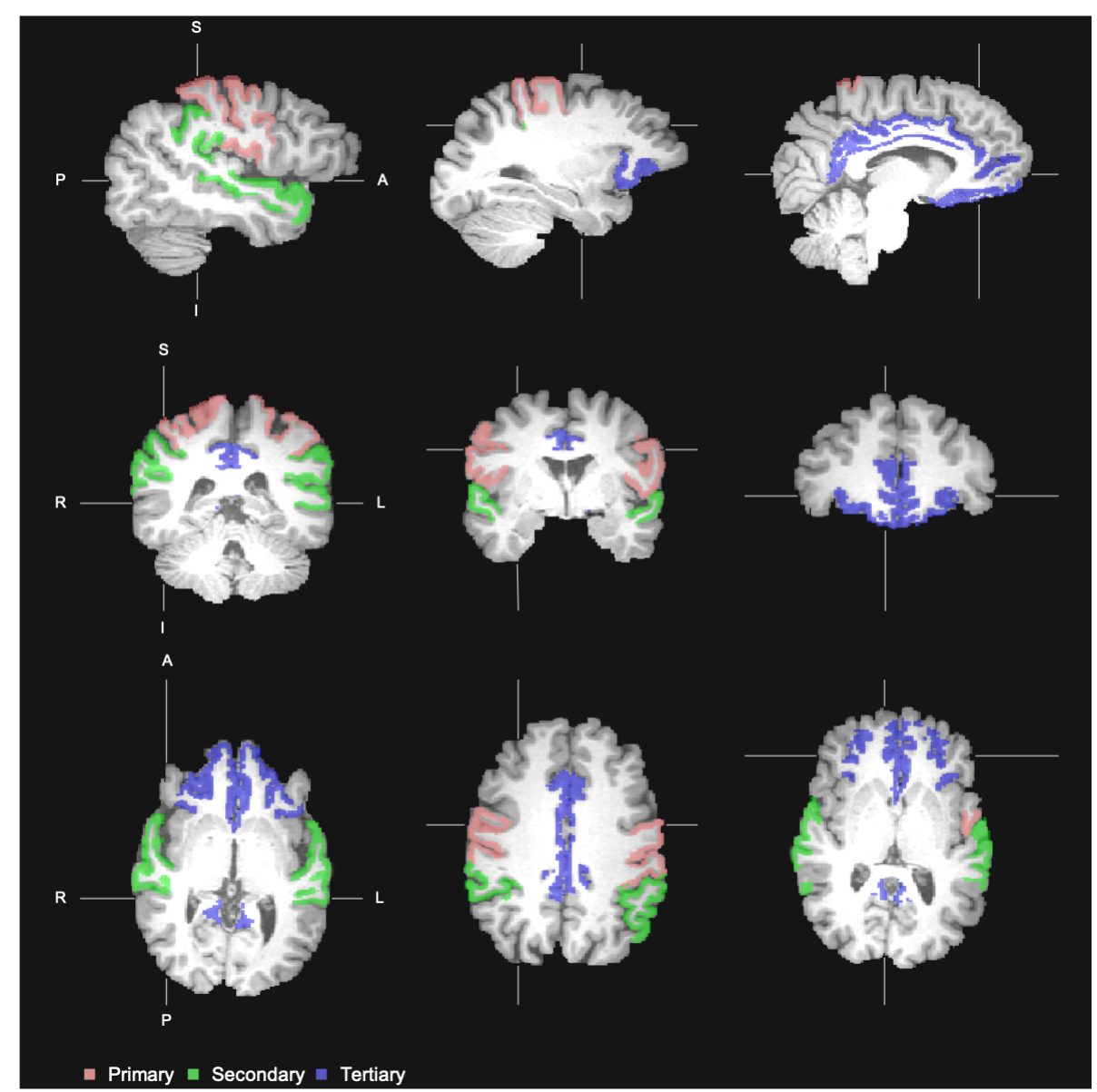

Figure 2.1. Cortical grey matter masks for the cortical regions of interest, with in pink: primary brain regions (precentral and postcentral gyrus), green: secondary brain regions (supramarginal and superior temporal gyrus), and blue: tertiary brain regions (orbitofrontal and cingulate cortex).

\subsubsection{DCE MRI processing}

For both the fast and slow DCE MRI, a reference image was created using the average of the pre-contrast images in each sequence. The slow DCE MRI data was motion corrected, and the fast DCE MRI data was motion-corrected and co-registered to the slow DCE MRI data using a single transformation. The reference slow DCE MRI was registered on the T1-weighted anatomic reference images, and the inverse of the obtained transformation matrix was used to transform the T1-weighted data, including the primary, secondary and tertiary brain regions, to slow DCE space.

At least 20 voxels in the superior sagittal sinus were manually selected (I.C.M.V.) to obtain a vascular input function (VIF) for each participant $(34,35)$. Signal-toconcentration conversion was applied to the VIF using in vitro data (diluted $\mathrm{MnCl}_{2}$ stock solution with varying gadobutrol concentrations ranging from 1 to $40 \mathrm{mM}$ and baseline 
T1 relaxation time comparable to human blood) and in the brain tissue by assuming a linear relationship and using the tissue relaxation time from the $\mathrm{T}_{10}$-map (55).

\subsubsection{Pharmacokinetic model analyses}

Using the participant-specific VIF, the Patlak approach (56) was used for voxel-wise pharmacokinetic modelling of the contrast agent concentration in brain tissue and blood plasma. The Patlak model was found to be a suitable model for brain tissue and is the most parsimonious, as it assumes no reflux from the brain tissue back to the blood (57).

With this approach, an estimation of the $\mathrm{K}_{\mathrm{i}}$-parameter [ $\left.\mathrm{min}^{-1}\right]$ was obtained for each voxel, which gives an indication of the leakage rate from the blood plasma to the brain tissue as a measure of permeability. Histograms of the $\mathrm{K}_{\mathrm{i}}$ values in the white and grey matter and primary, secondary and tertiary brain regions were calculated and corrected for noise (31), after which the mean $K_{i}$ was calculated for each region.

\subsection{7. $\quad$ Statistics}

The mean $K_{i}$ values appeared non-normally distributed and were therefore cube roottransformed to obtain a normal distribution. Multiple linear regression was performed with the mean $\mathrm{K}_{\mathrm{i}}$ in each ROI as dependent variable and age and sex as predictors, to investigate the association between $\mathrm{BBB}$ leakage and age while controlling for sex differences. Potential confounders, namely mean systolic blood pressure, BMI, diabetes and smoking habits, were also included in subsequent analyses to check whether they altered the results.

As additional post-hoc analyses, the influence of other measures of structural brain integrity, namely WMH volume, cortical thickness and hippocampal volume, was investigated. WMH volume values were log-transformed to obtain a normal distribution. Multiple linear regression was performed with $\mathrm{K}_{\mathrm{i}}$ in each ROI as dependent variable, while age, sex, one of the brain integrity measures and the interaction term between this measure and age were included as predictors. A non-significant interaction term was removed to test the significance of main effects.

All statistical analyses used a significance level of $\mathrm{p}<.05$ and were performed with commercial software (SPSS, version 24.0, IBM Corp., Armonk, NY, USA). 
Chapter 2

\subsection{Results}

The participant characteristics are presented in Table 2.1, showing high MMSE scores and low Fazekas and GCA ratings.

Table 2.1. Participant $(n=57)$ characteristics

\begin{tabular}{llc}
\hline & $\begin{array}{l}\text { Mean } \\
\text { (standard deviation) / percentage } / \text { median }\left(25^{\text {th }}-75^{\text {th }} \text { percentile) }\right.\end{array}$ \\
\hline Age & $65.8(10.2)$ & \\
\% Male & 52.6 & $15.8 / 54.4 / 29.8$ \\
\% Level of education & $1 / 2 / 3$ & \\
MMSEb $^{\text {a }}$ & $29.0(28.0-30.0)$ & $5.3 / 70.2 / 12.3 / 12.3$ \\
\% WMH Fazekas $^{c}$ & $0 / 1 / 2 / 3$ & $19.3 / 50.9 / 24.6 / 5.3$ \\
\% GCAd $^{\text {d }}$ & $0 / 1 / 2 / 3$ & \\
Systolic blood pressure & $141.3(17.2)$ & \\
\% Diabetes & 17.5 & \\
BMI & $27.7(4.5)$ & \\
\% Smoker & 14.0 & \\
eGFR & $69.5(12.6)$ &
\end{tabular}

a: Level of education: 1 = at most primary or lower vocational education; 2 = secondary education; 3 = higher vocational or scientific education

b: Mini-Mental State Examination (43): maximum score $=30$, cognitively normal $\geq 25$

c: Fazekas scale with a visual rating score of white matter hyperintensity load (45): $0=$ absent: none or a single punctuate WMH lesion; 1 = mild: multiple punctuate lesions; 2 = moderate: beginning of confluency of lesions; 3 = severe: large confluent lesions

d: Global cortical atrophy visual rating scale (46): $0=$ absent: normal volume/no ventricular enlargement; 1 = mild: opening of sulci/mild ventricular enlargement; 2 = moderate: volume loss of gyri/moderate ventricular enlargement; 3 = severe: 'knife blade' atrophy/severe ventricular enlargement

e: High blood pressure $>140 \mathrm{mmHg}$

For each ROI, the median and $25^{\text {th }}-7^{\text {th }}$ percentiles of the leakage rate and its association with age are presented in Table 2.2. 
Table 2.2. Median and $25^{\text {th }}-75^{\text {th }}$ percentiles of the leakage rate in the regions of interest, including the standardized regression coefficient with age

\begin{tabular}{llll}
\hline ROI & $\begin{array}{l}\text { Median leakage rate } \\
\left(\cdot 10^{-7} \mathrm{~min}^{-1}\right)\end{array}$ & $\begin{array}{l}\mathbf{2 5}-\mathbf{7 5} \text { percentiles } \\
\left(\cdot 10^{-7} \mathrm{~min}^{-1}\right)\end{array}$ & $\boldsymbol{\beta}^{\mathbf{a}}$ \\
\hline White matter & 11.4 & $5.1-20.4$ & $.306^{*}$ \\
Grey matter & 9.5 & $3.9-17.3$ & $.286^{*}$ \\
\hline Primary regions & 4.4 & $1.0-10.8$ & .145 \\
Secondary regions & 3.4 & $0.4-7.1$ & .098 \\
Tertiary regions & 9.2 & $1.1-25.1$ & $.307^{*}$ \\
\hline
\end{tabular}

a $\beta=$ standardized regression coefficient with age corrected for sex

* significant $(\mathrm{p}<.05)$

$\mathrm{K}_{\mathrm{i}}$ was significantly correlated with age, as older participants had significantly more widespread BBB leakage in the cerebral white matter $(\beta=.306, p=.024)$ and grey matter $(\beta=.286, \mathrm{p}=.035)$ (Table 2.2, Figure 2.2).
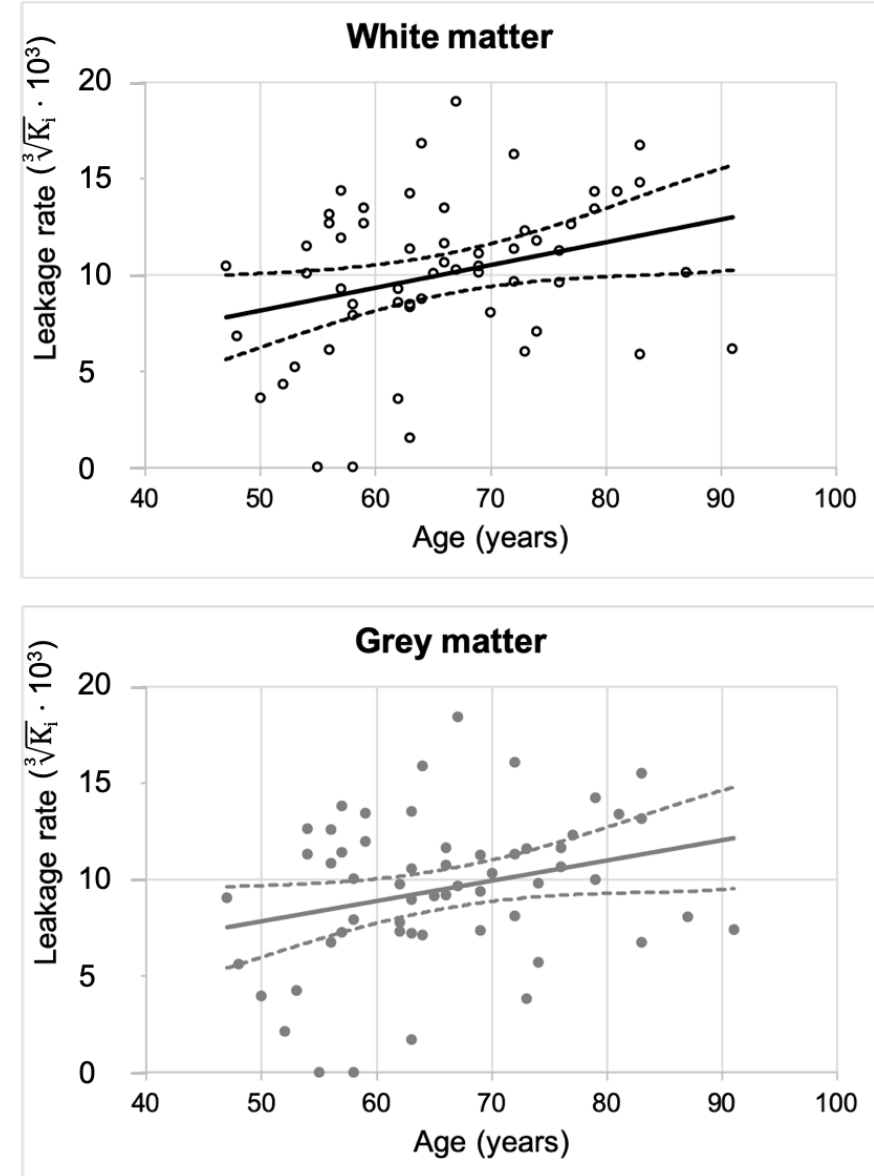

Figure 2.2. Scatterplots and linear regression of age and leakage rate in the white matter (upper) and grey matter (lower). 
From the three brain region classes, only $\mathrm{K}_{\mathrm{i}}$ in the tertiary brain regions was significantly correlated with age $(\beta=.301, p=.023)$ (Table 2.2, Figure 2.3).
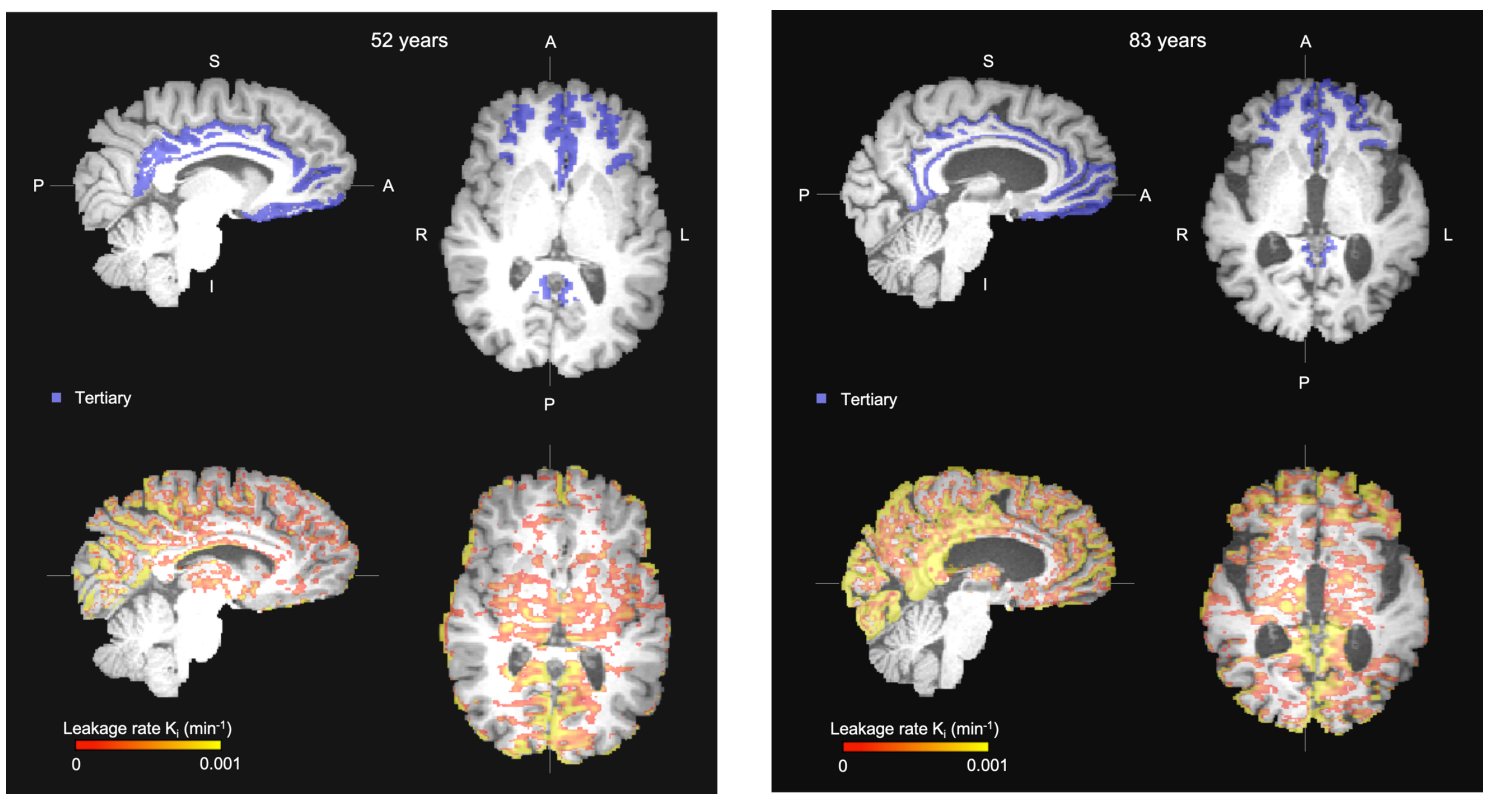

Figure 2.3. Upper: regions of interest of the tertiary brain regions (in blue), lower: the white and grey matter leakage maps of a younger participant (left; 52 years) and an older participant (right; 83 years). Note the stronger leakage in the older participant, especially in the cingulum.

The associations between $\mathrm{K}_{\mathrm{i}}$ and age remained significant when adding the potential confounders mean systolic blood pressure, diabetes, BMI and smoking habits to the regression analysis.

In the white and grey matter, as well as the tertiary brain regions, significant associations between $\mathrm{K}_{\mathrm{i}}$ and age disappeared after correction for WMH volume or cortical thickness. However, significant associations did not depend on hippocampal volume, as correcting for hippocampal volume did not affect the results.

\subsection{Discussion}

In this study, we hypothesized that BBB disruption would be associated with age in cognitively and neurologically healthy, middle-aged to older individuals. Our results confirmed this hypothesis, as we found higher BBB leakage rate in older individuals from a healthy group when investigating the total cerebral white and grey matter tissue 
compartments. Also, we found a significant correlation between BBB leakage and age in the tertiary brain regions, but not in the primary or secondary regions, which confirms that $\mathrm{BBB}$ disruption increases with age especially in those regions that are known to be most vulnerable to normal age-related deterioration.

Previous studies have demonstrated that decrease in cerebral blood flow and cortical volume reductions are most pronounced in the tertiary brain regions during aging $(58,59)$, which supports that age-related detrimental processes especially affect tertiary regions. Our study now shows that BBB disruption could be one of these processes, and strongly supports the notion that BBB disruption is an underlying mechanism of normal age-related neurophysiological decline.

Previous studies have already demonstrated that white matter integrity loss and cortical thinning are part of the normal aging process $(60,61)$. We now have evidence that $\mathrm{BBB}$ disruption increases during normal aging, but this association is largely explained by age-related white matter integrity loss and decrease in cortical thickness. White matter integrity loss, cortical thinning and BBB disruption all seem to be normal physiological aging phenomena, while hippocampal volume loss is possibly an independent process that might reflect pathology such as found in AD (62). For future studies, it would be interesting to also include a measure of regional cerebral blood flow, to investigate how cerebral blood flow reduction correlates with these other age-related processes.

Gaining more knowledge on increase in BBB leakage during normal aging could improve the interpretation and value estimation of leakage rate in pathological conditions. Previous studies have already associated BBB disruption with neurodegenerative disorders, such as AD. It seems reasonable to assume that the distinction between normal and pathological conditions is not a dichotomous, but a gradual phenomenon, and therefore normal aging individuals most likely still experience some degree of age-related setback. Our finding that BBB leakage is even higher in older adults within the range of normal aging implies that BBB disruption could be an early event in the pathological cascade that in some individuals may eventually be contributing to a neurodegenerative disorder. To gain even better insight into BBB disruption over the age span, studies should be conducted over a broader age range. Including adults around the ages of 20 and 30 years could yield a reference point for BBB leakage at later ages. 
Our study uses a cross-sectional design to investigate whether higher leakage rate is associated with older age, so ideally future studies should conduct measurements over time to see whether BBB leakage actually increases as people age. Only longitudinal studies might give information on causality. White matter pathology might damage the vessel walls and generate BBB disruption. However, not only the WMHs, but also their nearby normal-appearing white matter has been found to be more permeable, which disputes this notion and could mean that BBB disruption is an initiating factor of the pathological cascade, which triggers white matter pathology (63-65).

One could also argue on how to define normal aging. Normal aging often means usual aging, where individuals still experience some age-related setback typical for their age group and may have comorbid conditions, as long as these conditions do not significantly affect their functioning $(66,67)$. Our sample for instance had a high mean systolic blood pressure (Table 2.1). However, these values are not unusual for these age groups (68) and might be an overestimation, as anxiety while measurements are conducted might elevate blood pressure (69). Also, correcting for potential confounders, such as high systolic blood pressure and BMI, did not change our results. Ideally, future studies should also investigate the relation between cardiovascular disease, BBB disruption and age. For instance, heart failure, which may result in increased central venous pressure, has been shown to cause BBB disruption (70). Moreover, we selected participants from the Maastricht Aging Study, who have participated in this study for 25 years. Participants who are willing and able to participate over such a long period of time are more likely to represent those with an above-average health condition. We were able to find significantly more BBB leakage with older age even within this functionally healthy subsample, and this effect will possibly be even stronger in a more diverse group, so we expect our findings to be relevant for the general aging population.

The integrity of the cerebral vasculature is an important factor in the development of neurodegenerative disorders. Prevention strategies could therefore focus on promoting neurovascular health. Studies are often aimed at the removal of neurotoxic proteins, but also identification of methods to maintain BBB integrity already at an early stage, such as the promising work on restoring $\mathrm{NAD}^{+}$levels, might eventually help increasing the proportion of people who will age in a healthier fashion. 


\subsection{Conclusion}

We found higher BBB leakage rate with older age in healthy individuals, especially in those brain regions most vulnerable to age-related deterioration, which supports the notion that BBB disruption could be an underlying mechanism of normal age-related decline. Promoting neurovascular health and identification of methods to maintain BBB integrity could be a promising avenue to promote healthy aging.

\subsection{Acknowledgements}

The authors would like to acknowledge the participants for their contribution to scientific research and Jos M.G.M. Slenter and Jan H. Jungerius for ongoing software, hardware and network support.

\subsection{Declaration of interest}

The authors declare that they have no competing interests.

\subsection{Ethical approval and consent}

The study was approved beforehand by the medical ethics review committee of Maastricht University Medical Center (approval number: METC163050). All participants provided written informed consent beforehand regarding participation and publication of the data.

\subsection{References}

1. Lutz W, Sanderson W, Scherbov S. The coming acceleration of global population ageing. Nature. 2008;451(7179):716-9.

2. Woodruff-Pak DS. The neuropsychology of aging: Blackwell Publishing; 1997.

3. Rapp PR, Amaral DG. Individual differences in the cognitive and neurobiological consequences of normal aging. Trends in neurosciences. 1992;15(9):340-5.

4. Farkas E, Luiten PG. Cerebral microvascular pathology in aging and Alzheimer's disease. Progress in neurobiology. 2001;64(6):575-611.

5. Norton E, Bridges L, Kenyon L, Esiri M, Bennett D, Hainsworth A. Cell Senescence and Cerebral Small Vessel Disease in the Brains of People Aged 80 Years and Older. Journal of Neuropathology and Experimental Neurology. 2019;78.

6. Zlokovic BV. Neurovascular pathways to neurodegeneration in Alzheimer\&\#39;s disease and other disorders. Nature Reviews Neuroscience. 2011;12:723. 


\section{Chapter 2}

7. Zhao Z, Nelson AR, Betsholtz C, Zlokovic BV. Establishment and dysfunction of the blood-brain barrier. Cell. 2015;163(5):1064-78.

8. Montagne A, Zhao Z, Zlokovic BV. Alzheimer's disease: A matter of blood-brain barrier dysfunction? Journal of Experimental Medicine. 2017;214(11):3151-69.

9. Zlokovic BV. The blood-brain barrier in health and chronic neurodegenerative disorders. Neuron. 2008;57(2):178-201.

10. Ungvari Z, Tarantini S, Donato AJ, Galvan V, Csiszar A. Mechanisms of vascular aging. Circulation research. 2018;123(7):849-67.

11. Murugesan N, Demarest TG, Madri JA, Pachter JS. Brain regional angiogenic potential at the neurovascular unit during normal aging. Neurobiology of aging. 2012;33(5):1004. e1-. e16.

12. Ungvari Z, Tucsek Z, Sosnowska D, Toth P, Gautam T, Podlutsky A, et al. Aging-induced dysregulation of dicer1-dependent microRNA expression impairs angiogenic capacity of rat cerebromicrovascular endothelial cells. Journals of Gerontology Series A: Biomedical Sciences and Medical Sciences. 2013;68(8):877-91.

13. Yoshino J, Baur JA, Imai S-i. NAD+ intermediates: the biology and therapeutic potential of NMN and NR. Cell metabolism. 2018;27(3):513-28.

14. Gomes AP, Price NL, Ling AJ, Moslehi JJ, Montgomery MK, Rajman L, et al. Declining NAD+ induces a pseudohypoxic state disrupting nuclear-mitochondrial communication during aging. Cell. 2013;155(7):1624-38.

15. Csiszar A, Tarantini S, Yabluchanskiy A, Balasubramanian P, Kiss T, Farkas E, et al. Role of endothelial NAD+ deficiency in age-related vascular dysfunction. American Journal of Physiology-Heart and Circulatory Physiology. 2019;316(6):H1253-H66.

16. Bonkowski MS, Sinclair DA. Slowing ageing by design: the rise of NAD+ and sirtuin-activating compounds. Nature reviews Molecular cell biology. 2016;17(11):679.

17. Kiss T, Balasubramanian P, Valcarcel-Ares MN, Tarantini S, Yabluchanskiy A, Csipo T, et al. Nicotinamide mononucleotide (NMN) treatment attenuates oxidative stress and rescues angiogenic capacity in aged cerebromicrovascular endothelial cells: a potential mechanism for the prevention of vascular cognitive impairment. GeroScience. 2019;41(5):619-30.

18. Tarantini S, Yabluchanskiy A, Csipo T, Fulop G, Kiss T, Balasubramanian P, et al. Treatment with the poly (ADP-ribose) polymerase inhibitor PJ-34 improves cerebromicrovascular endothelial function, neurovascular coupling responses and cognitive performance in aged mice, supporting the NAD+ depletion hypothesis of neurovascular aging. GeroScience. 2019;41(5):533-42.

19. Mills KF, Yoshida S, Stein LR, Grozio A, Kubota S, Sasaki Y, et al. Long-term administration of nicotinamide mononucleotide mitigates age-associated physiological decline in mice. Cell metabolism. 2016;24(6):795-806.

20. Zhang H, Ryu D, Wu Y, Gariani K, Wang X, Luan P, et al. NAD+ repletion improves mitochondrial and stem cell function and enhances life span in mice. Science. 2016;352(6292):1436-43.

21. Raja R, Rosenberg GA, Caprihan A. MRI measurements of Blood-Brain Barrier function in dementia: A review of recent studies. Neuropharmacology. 2018;134:259-71. 
22. Fernando MS, Simpson JE, Matthews F, Brayne C, Lewis CE, Barber R, et al. White matter lesions in an unselected cohort of the elderly. Stroke. 2006;37(6):1391-8.

23. Levit A, Hachinski V, Whitehead SN. Neurovascular unit dysregulation, white matter disease, and executive dysfunction: the shared triad of vascular cognitive impairment and Alzheimer disease. GeroScience. 2020:1-21.

24. Burgmans S, van de Haar HJ, Verhey FR, Backes WH. Amyloid- $\beta$ interacts with blood-brain barrier function in dementia: a systematic review. Journal of Alzheimer's Disease. 2013;35(4):859-73.

25. Iadecola C, Zhang F, Niwa K, Eckman C, Turner SK, Fischer E, et al. SOD1 rescues cerebral endothelial dysfunction in mice overexpressing amyloid precursor protein. Nature Neuroscience. 1999;2:157.

26. Niwa K, Kazama K, Younkin L, Younkin SG, Carlson GA, Iadecola C. Cerebrovascular autoregulation is profoundly impaired in mice overexpressing amyloid precursor protein. American Journal of PhysiologyHeart and Circulatory Physiology. 2002;283(1):H315-H23.

27. Niwa K, Kazama K, Younkin SG, Carlson GA, Iadecola C. Alterations in Cerebral Blood Flow and Glucose Utilization in Mice Overexpressing the Amyloid Precursor Protein. Neurobiology of Disease. 2002;9(1):61-8.

28. DiBattista AM, Sierra F, Masliah E. NIA workshop on senescence in brain aging and Alzheimer's disease and its related dementias. Springer; 2020.

29. Montagne A, Barnes SR, Sweeney MD, Halliday MR, Sagare AP, Zhao Z, et al. Blood-brain barrier breakdown in the aging human hippocampus. Neuron. 2015;85(2):296-302.

30. Montagne A, Nation DA, Pa J, Sweeney MD, Toga AW, Zlokovic BV. Brain imaging of neurovascular dysfunction in Alzheimer's disease. Acta neuropathologica. 2016;131(5):687-707.

31. van de Haar HJ, Burgmans S, Jansen JF, van Osch MJ, van Buchem MA, Muller M, et al. Blood-brain barrier leakage in patients with early Alzheimer disease. Radiology. 2016;281(2):527-35.

32. Iturria-Medina Y, Sotero RC, Toussaint PJ, Mateos-Pérez JM, Evans AC, The Alzheimer's Disease Neuroimaging I, et al. Early role of vascular dysregulation on late-onset Alzheimer's disease based on multifactorial data-driven analysis. Nature Communications. 2016;7:11934.

33. Farrall AJ, Wardlaw JM. Blood-brain barrier: ageing and microvascular disease-systematic review and meta-analysis. Neurobiology of aging. 2009;30(3):337-52.

34. Jelescu IO, Leppert IR, Narayanan S, Araújo D, Arnold DL, Pike GB. Dual-temporal resolution dynamic contrast-enhanced MRI protocol for blood-brain barrier permeability measurement in enhancing multiple sclerosis lesions. Journal of Magnetic Resonance Imaging. 2011;33(6):1291-300.

35. van de Haar HJ, Jansen JFA, Jeukens CRLPN, Burgmans S, Buchem MA, Muller M, et al. Subtle bloodbrain barrier leakage rate and spatial extent: Considerations for dynamic contrast-enhanced MRI. Medical Physics. 2017;44(8):4112-25.

36. Wang H, Golob EJ, Su M-Y. Vascular volume and blood-brain barrier permeability measured by dynamic contrast enhanced MRI in hippocampus and cerebellum of patients with MCI and normal controls. Journal of Magnetic Resonance Imaging. 2006;24(3):695-700.

37. Zhang CE, Wong SM, van de Haar HJ, Staals J, Jansen JF, Jeukens CR, et al. Blood-brain barrier leakage is more widespread in patients with cerebral small vessel disease. Neurology. 2017;88(5):426-32. 


\section{Chapter 2}

38. Pantoni L. Cerebral small vessel disease: from pathogenesis and clinical characteristics to therapeutic challenges. The Lancet Neurology. 2010;9(7):689-701.

39. Nation DA, Sweeney MD, Montagne A, Sagare AP, D’Orazio LM, Pachicano M, et al. Blood-brain barrier breakdown is an early biomarker of human cognitive dysfunction. Nature Medicine. 2019.

40. Andrews-Hanna JR, Snyder AZ, Vincent JL, Lustig C, Head D, Raichle Marcus E, et al. Disruption of Large-Scale Brain Systems in Advanced Aging. Neuron. 2007;56(5):924-35.

41. Casey BJ, Tottenham N, Liston C, Durston S. Imaging the developing brain: what have we learned about cognitive development? Trends in Cognitive Sciences. 2005;9(3):104-10.

42. Jolles J, Houx P, Van Boxtel M, Ponds R. Maastricht aging study: Determinants of cognitive aging: Neuropsych Publishers Maastricht; 1995.

43. Folstein MF, Folstein SE, McHugh PR. "Mini-mental state": a practical method for grading the cognitive state of patients for the clinician. Journal of psychiatric research. 1975;12(3):189-98.

44. Gélinas I, Gauthier L, McIntyre M, Gauthier S. Development of a functional measure for persons with Alzheimer's disease: the disability assessment for dementia. American Journal of Occupational Therapy. 1999;53(5):471-81.

45. Fazekas F, Kleinert R, Offenbacher H, Schmidt R, Kleinert G, Payer F, et al. Pathologic correlates of incidental MRI white matter signal hyperintensities. Neurology. 1993;43(9):1683-.

46. Pasquier F, Leys D, Weerts JG, Mounier-Vehier F, Barkhof F, Scheltens P. Inter-and intraobserver reproducibility of cerebral atrophy assessment on MRI scans with hemispheric infarcts. European neurology. 1996;36(5):268-72.

47. Rapoport SI. Opening of the blood-brain barrier by acute hypertension. Experimental neurology. 1976;52(3):467-79.

48. Gustafson D, Karlsson C, Skoog I, Rosengren L, Lissner L, Blennow K. Mid-life adiposity factors relate to blood-brain barrier integrity in late life. Journal of internal medicine. 2007;262(6):643-50.

49. Starr J, Wardlaw J, Ferguson K, MacLullich A, Deary I, Marshall I. Increased blood-brain barrier permeability in type II diabetes demonstrated by gadolinium magnetic resonance imaging. Journal of Neurology, Neurosurgery \& Psychiatry. 2003;74(1):70-6.

50. Mazzone P, Tierney W, Hossain M, Puvenna V, Janigro D, Cucullo L. Pathophysiological impact of cigarette smoke exposure on the cerebrovascular system with a focus on the blood-brain barrier: expanding the awareness of smoking toxicity in an underappreciated area. International journal of environmental research and public health. 2010;7(12):4111-26.

51. Larsson HB, Courivaud F, Rostrup E, Hansen AE. Measurement of brain perfusion, blood volume, and blood-brain barrier permeability, using dynamic contrast-enhanced T1-weighted MRI at 3 tesla. Magnetic Resonance in Medicine: An Official Journal of the International Society for Magnetic Resonance in Medicine. 2009;62(5):1270-81.

52. Jacobs HI, Clerx L, Gronenschild EH, Aalten P, Verhey FR. White matter hyperintensities are positively associated with cortical thickness in Alzheimer's disease. Journal of Alzheimer's Disease. 2014;39(2):409-22.

53. Fischl B, Sereno MI, Dale AM. Cortical Surface-Based Analysis: II: Inflation, Flattening, and a Surface-Based Coordinate System. NeuroImage. 1999;9(2):195-207. 
54. Desikan RS, Ségonne F, Fischl B, Quinn BT, Dickerson BC, Blacker D, et al. An automated labeling system for subdividing the human cerebral cortex on MRI scans into gyral based regions of interest. Neuroimage. 2006;31(3):968-80.

55. van de Haar HJ, Jansen JF, van Osch MJ, van Buchem MA, Muller M, Wong SM, et al. Neurovascular unit impairment in early Alzheimer's disease measured with magnetic resonance imaging. Neurobiology of aging. 2016;45:190-6.

56. Patlak CS, Blasberg RG, Fenstermacher JD. Graphical evaluation of blood-to-brain transfer constants from multiple-time uptake data. J Cereb Blood Flow Metab. 1983;3(1):1-7.

57. Cramer SP, Larsson HB. Accurate determination of blood-brain barrier permeability using dynamic contrast-enhanced T1-weighted MRI: a simulation and in vivo study on healthy subjects and multiple sclerosis patients. Journal of Cerebral Blood Flow \& Metabolism. 2014;34(10):1655-65.

58. Martin AJ, Friston KJ, Colebatch JG, Frackowiak RS. Decreases in regional cerebral blood flow with normal aging. Journal of Cerebral Blood Flow \& Metabolism. 1991;11(4):684-9.

59. Fjell AM, McEvoy L, Holland D, Dale AM, Walhovd KB. Brain Changes in Older Adults at Very Low Risk for Alzheimer's Disease. The Journal of Neuroscience. 2013;33(19):8237-42.

60. Thambisetty M, Wan J, Carass A, An Y, Prince JL, Resnick SM. Longitudinal changes in cortical thickness associated with normal aging. Neuroimage. 2010;52(4):1215-23.

61. Maniega SM, Hernández MCV, Clayden JD, Royle NA, Murray C, Morris Z, et al. White matter hyperintensities and normal-appearing white matter integrity in the aging brain. Neurobiology of aging. 2015;36(2):909-18.

62. Wang L, Swank JS, Glick IE, Gado MH, Miller MI, Morris JC, et al. Changes in hippocampal volume and shape across time distinguish dementia of the Alzheimer type from healthy aging $\boldsymbol{z}^{2}$. Neuroimage. 2003;20(2):667-82.

63. Riphagen JM, Gronenschild EHBM, Salat DH, Freeze WM, Ivanov D, Clerx L, et al. Shades of white: diffusion properties of T1- and FLAIR-defined white matter signal abnormalities differ in stages from cognitively normal to dementia. Neurobiology of Aging. 2018;68:48-58.

64. Wong SM, Jansen JF, Zhang CE, Hoff EI, Staals J, van Oostenbrugge RJ, et al. Blood-brain barrier impairment and hypoperfusion are linked in cerebral small vessel disease. Neurology. 2019;92(15):e1669e77.

65. Hainsworth A. White matter lesions in cerebral small vessel disease: Underperfusion or leaky vessels? Neurology. 2019;92:10.1212/WNL.0000000000007258.

66. Petersen RC, Smith GE, Waring SC, Ivnik RJ, Kokmen E, Tangelos EG. Aging, memory, and mild cognitive impairment. International psychogeriatrics. 1997;9(S1):65-9.

67. Schaie KW. The course of adult intellectual development. American psychologist. 1994;49(4):304.

68. Gaillard C, Jolles J, van Boxtel MP, van Es PN, de Leeuw PW. Repeated automatic versus ambulatory blood pressure measurement: the effects of age and sex in a normal ageing population. Journal of hypertension. 1996;14(1):31-40.

69. Verdecchia P, Staessen JA, White W, Imai Y, O'Brien E. Properly defining white coat hypertension. European heart journal. 2002;23(2):106-9. 


\section{Chapter 2}

70. Fulop GA, Ahire C, Csipo T, Tarantini S, Kiss T, Balasubramanian P, et al. Cerebral venous congestion promotes blood-brain barrier disruption and neuroinflammation, impairing cognitive function in mice. Geroscience. 2019;41(5):575-89. 




\section{Chapter 3}

\section{Imaging the role of blood-brain barrier disruption in normal cognitive aging}

GeroScience 42, 1751-1764 (2020). https://doi.org/10.1007/s11357-020-00282-1

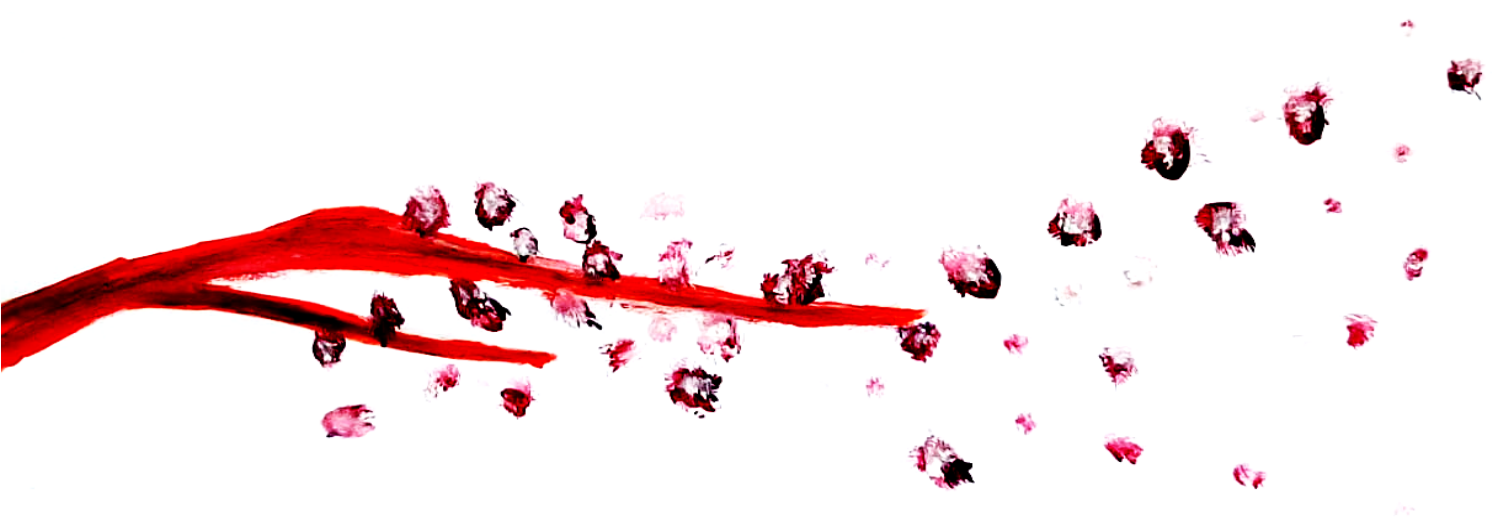


Chapter 3

\subsection{Abstract}

Objective

To investigate whether blood-brain barrier (BBB) disruption is a potential mechanism of usual age-related cognitive decline, we conducted dynamic-contrast enhanced (DCE) MRI to measure BBB leakage in a healthy sample, and investigated the association with longitudinal cognitive decline.

\section{Methods}

In a sample of neurologically and cognitively healthy, older individuals, BBB leakage rate in the white and grey matter and hippocampus was measured using DCE MRI with pharmacokinetic modelling. Regression analysis was performed to investigate whether the leakage rate was associated with decline in cognitive performance (memory encoding, memory retrieval, executive functioning and processing speed) over 12 years. Results

White and grey matter BBB leakage were significantly associated with decline in memory retrieval. No significant relations were found between hippocampal BBB leakage and cognitive performance.

\section{Conclusions}

BBB disruption already being associated with usual cognitive aging, supports that this neurovascular alteration is a possible explanation for the cognitive decline inherent to the aging process. More insight into BBB leakage during the normal aging process could improve estimation and interpretation of leakage rate in pathological conditions. The current results might also stimulate the search for strategies to maintain BBB integrity and help increase the proportion people experiencing successful aging.

Netherlands Trial Register number: NL6358, date of registration: 2017-03-24

\section{Keywords}

Blood-brain barrier, cognitive aging, dynamic contrast-enhanced MRI, cerebrovascular dysfunction, successful aging 


\subsection{Introduction}

Over the last years, population demographics have been shifting towards a larger proportion of older individuals, due to a combination of decreasing birth rates and increasing life expectancy (1). With a larger older population, determining which factors could lead towards successful aging has become an important scientific objective even more. Besides the absence of disease and disability, an important component of successful aging is the maintenance of cognitive functioning (2). An aging population is increasingly confronted with age-related neurobiological changes, and such changes in brain structure and function are often associated with changes in cognition $(3,4)$. However, aging does not necessarily lead to cognitive impairment, as some people age without considerable cognitive setback, while others show substantial cognitive decline (5). Investigating what neurobiological changes lead to age-related cognitive decline and hinder successful aging is becoming increasingly important $(4,6)$.

Age has a large impact on the cerebrovascular system and is a major risk factor for cerebrovascular damage $(7,8)$. Cerebrovascular disease is therefore a highly prevalent age-related condition (9). Moreover, cerebrovascular disease appears to affect cognition even in elderly in the normal aging spectrum (10). The blood brain barrier (BBB) is part of the cerebrovascular system and was shown to be vulnerable to age-related changes $(9$, 11, 12). Recently, higher BBB permeability has been linked to an increasing number of age-related neurological disorders $(8,13,14)$. Previous studies were even able to find an association between cognitive impairment inherent to these conditions and BBB disruption $(8,15-17)$.

At the level of the microvascular network, specialized cells work together in a neurovascular unit (NVU) to maintain cerebral blood flow (CBF), oxygen delivery and energy supply (18). The NVU consists of vascular cells (i.e. endothelial cells), glial cells (i.e. astrocytes) and neurons $(18,19)$. Interaction between vascular cells and neurons in the NVU creates hemodynamic coupling, allowing an increase in neuronal activation to be followed by an appropriate CBF response $(20,21)$. The endothelial cells are connected by tight junctions to create a strong barrier forming the basis of the BBB (18). The BBB separates blood plasma from brain tissue and regulates the delivery of energy metabolites and nutrients to the neurons, while preventing neurotoxins from entering the brain. 
The endothelium allows small molecules as oxygen and carbon dioxide to rapidly and freely diffuse across the BBB, which is essential for brain metabolism and $\mathrm{pH}$ regulation (22). Small, lipophilic molecules can also cross the BBB (23). As for the rest, the endothelium contains specialized transport systems using active efflux, carriermediated transport and receptor-mediated transport to enable nutrients to pass from blood plasma to brain tissue and to remove waste products from the brain $(22,24)$. The BBB thereby protects the brain from ion fluctuations that occur in the blood after a meal or exercise and ensures homeostasis is maintained, which is essential for neuronal and synaptic functioning $(25,26)$.

Neurovascular dysfunction has been suggested to initiate the cascade leading to neurodegeneration and $\operatorname{AD}(18,25,27)$. Neurovascular dysfunction involves uncoupling of the NVU, which can lead reduction and dysregulation of CBF and disruption of the BBB $(28,29)$. A recent hypothesis for instance states that depletion of the nicotinamide adenine dinucleotide $\left(\mathrm{NAD}^{+}\right)$substrate can lead to endothelial dysfunction, which impairs the integrity of the cerebral microcirculation (30). Both CBF and BBB disruption impair the supply of oxygen and nutrients to the neurons. As a result, hypoxia and inflammation may occur and trigger subsequent pathological processes, which make the brain vulnerable to neuronal dysfunction, and eventually neurodegeneration $(8,31,32)$. Moreover, BBB disruption reduces the clearance of interstitial solutes from the brain and could lead to accumulation of toxic waste products, such as the amyloid- $\beta$ protein $(A \beta)$ in case of $A D$ (33). In turn, $A \beta$ can further impair neurovascular functioning, including $C B F$ regulation and BBB integrity. These neurovascular alterations occur even before the formation of $A \beta$ plaques, suggesting that they are an early event in the pathological cascade (34-36), which is supported by several imaging studies demonstrating early BBB disruption in the development of $\operatorname{AD}(15,37-39)$. Studies have even shown that carrying the $\varepsilon 4$ allelle of the apolipoprotein E gene (APOE4), indicating genetic susceptiblility to $\mathrm{AD}(40)$, is associated with accelerated BBB disruption (41-43).

Thus, BBB disruption is considered as an early event in the pathological cascade possibly leading to neurodegeneration $(15,38)$. Early BBB leakage was also associated with global cognitive functioning, as leakage rates in individuals with early $\mathrm{AD}$ and healthy age-matched controls correlated significantly with cognitive function (MiniMental State Examination: MMSE (44)) (15). Moreover, individuals with early cognitive dysfunction, not accounted for by a neurological disorder, demonstrated increased BBB 
Imaging the role of blood-brain barrier disruption in normal cognitive aging

disruption independent of $A \beta$ or tau (17). This suggests that BBB breakdown could already be present in those who experience age-related cognitive decline, even without having a neurological disorder. A recent study demonstrated that BBB disruption is not only more prominent in APOE4 carriers, but this effect was stronger in cognitively impaired individuals, which demonstrates that BBB disruption is involved in APOE4related cognitive decline (45). BBB disruption might be a contributing mechanism to cognitive aging.

Interest in BBB disruption is increasing due to recent discoveries about its role in brain pathology, but also due to recent advances made in the development of magnetic resonance imaging (MRI) techniques to measure permeability $(31,46)$. One of the most promising techniques is dynamic contrast-enhanced (DCE) MRI, which measures contrast agent leakage from the blood plasma to the brain interstitial space over time, and allows the detection of subtle leakage values, for instance in dementia (47). Using DCE MRI has made it possible to detect increased BBB leakage in mild cognitive impairment (MCI), the transition stage to AD (16), and small vessel disease (48), the most important cause of vascular dementia (49). Increased BBB leakage at older ages was even found in healthy individuals (50). However, whether BBB leakage is associated with variation in usual age-related cognitive decline remains to be investigated.

The current study used dual-time resolution DCE MRI to investigate whether BBB breakdown is already associated with cognitive decline in cognitively and neurologically healthy, older individuals. We hypothesized that BBB leakage would be significantly associated with usual age-related cognitive decline, which would support BBB disruption as contributing mechanism to cognitive aging.

\subsection{Methods}

\subsubsection{Participants}

57 participants (mean age: 66 years, age range: 47 - 91 years) were recruited from the Maastricht Aging Study (MAAS) (51). Cognitively and neurologically healthy participants were selected, based on the following criteria: MMSE (44) score $\geq 25$; Disabilities Assessment of Dementia (DAD (52)) score $\geq 90 \%$; as reported in their medical history: no diagnosis of dementia, prodromal dementia, MCI, or any other psychiatric or neurological condition, no large structural brain abnormalities, brain surgery or brain 
Chapter 3

trauma, and no cognitive impairment due to substance abuse; no contraindications for MRI scanning or gadolinium-based contrast agent (sufficiently functioning kidneys with glomerular filtration rate $(\mathrm{eGFR})>30 \mathrm{~mL} / \mathrm{min})$.

\subsubsection{Cognitive decline}

Participants performed a short battery of standardized cognitive tests. Memory function was measured using the verbal learning test (VLT (53)), in which the immediate recall score gives an indication of short-term episodic memory and learning, and the delayed recall score is considered to be a measure of long-term episodic memory. Processing speed was measured with the letter-digit substitution test (LDST (54)). Executive functioning was measured with the Stroop colour-word test (55), in which the interference score is considered to be a measure of inhibition.

Cognitive decline was calculated by subtracting the participants' current score from their previous score in the last measure of MAAS approximately 12 years ago, so that a larger difference score would correspond to more cognitive decline. The interference score of the Stroop test was obtained using the time participants needed to complete the task and was the only test in which a larger difference score indicated less decline. The difference score of Stroop interference was reversed by multiplying difference scores with -1 , so that a larger score would correspond to more cognitive decline.

\subsubsection{MRI acquisition}

Images were acquired on a 3-Tesla MRI scanner (Achieva TX, Philips Healthcare, Best, The Netherlands) with a 32-channel head coil. The imaging protocol included a 3D T1weighted sequence for anatomical reference, a 3D T2-weighted fluid attenuation inversion recovery (FLAIR) sequence for brain segmentation and a dual-time resolution dynamic contrast-enhanced (DCE) sequence for leakage calculations.

The fast sequence of the dual-time resolution DCE MRI had a dynamic scan interval of $3.2 \mathrm{~s}$ and consisted of 29 volumes with a voxel size of $2 \times 2 \times 5 \mathrm{~mm}$. During the fast sequence, the gadolinium-containing contrast agent $(0.1 \mathrm{mmol} / \mathrm{kg}$ gadobutrol, Gadavist $^{\circledR}$, Bayer AG, Leverkusen, Germany) was injected in the antecubital vein (injection rate $3 \mathrm{~mL} / \mathrm{sec}, 20 \mathrm{~mL}$ saline flush). After the rapid spread over the vasculature, a slow sequence was applied for leakage into the brain tissue. The slow sequence had a 
dynamic scan interval of $30.5 \mathrm{~s}$ and consisted of 30 volumes with a voxel size of $1 \times 1 \times 2$ $\mathrm{mm}$. Conversion of tissue signal intensity to contrast agent concentration was enabled by T1-mapping.

\subsection{4. $\quad$ Structural brain characteristics and segmentation}

T2-FLAIR images were also used to obtain scores on the Fazekas scale (56) for white matter hyperintensity (WMH) load, and T1-weighted images to obtain scores on the global cortical atrophy (GCA) scale (57) and the medial temporal lobe atrophy (MTA) scale (58). Categorical scores on these visual rating scales were assigned by experienced neuroradiologists (A.A.P and W.M.P.).

WMH volume $\left[\mathrm{cm}^{3}\right]$ was quantified using the in-house developed, semi-automated segmentation tool GIANT (59) and corrected for intracranial volume, as volumetric measures may be confounded by their correlation with head size (60).

Automated brain segmentations were created using FreeSurfer software (version 6.0.0 (61)), after which these segmentations were visually checked and manually adjusted (I.C.M.V.). From the FreeSurfer output, cortical thickness averaged over the whole brain $[\mathrm{mm}]$ and hippocampal volume averaged over the hemispheres $\left[\mathrm{cm}^{3}\right]$ were obtained, after which hippocampal volume was corrected for intracranial volume. For our analyses, total white matter, total grey matter (cortical and deep grey matter and hippocampus), and the hippocampus separately, were extracted to create tissue masks (61).

\subsubsection{DCE MRI processing}

Both the fast and slow DCE MRI were motion corrected and a reference image was created using the average of the pre-contrast images in each sequence. The fast DCE MRI data was co-registered on the slow DCE MRI, and the reference slow DCE MRI was registered on the T1-weighted images for anatomical reference. Ultimately, the inverse of the obtained transformation matrix was used to transform the T1-weighted data to slow DCE space.

An individual vascular input function (VIF) was obtained for each participant by manually (I.C.M.V.) selecting at least 20 voxels in the superior sagittal sinus. The tissue signal in the brain ROIs was converted to contrast concentration by assuming a linear relationship and using the tissue relaxation time from the $\mathrm{T}_{10}-\mathrm{map}$, while the blood 
Chapter 3

plasma signal in the VIF was converted to contrast concentration using in vitro-data (62) (diluted $\mathrm{MnCl}_{2}$ stock solution with varying gadobutrol concentrations ranging from 1 to $40 \mathrm{mM}$ and baseline $\mathrm{T} 1$ relaxation time comparable to human blood).

\subsubsection{Pharmacokinetic model analysis}

Voxel-wise pharmacokinetic modelling was applied to the contrast agent concentration time-curves in brain tissue and blood plasma. The most parsimonious model still giving a proper fit to the data was used, namely the Patlak model, which assumes no reflux from the brain tissue back to the blood and has been demonstrated to be suitable for brain tissue (63).

Applying the Patlak model to the contrast concentration time-curves gave us an estimation of the $\mathrm{K}_{\mathrm{i}}$-parameter $\left[\mathrm{min}^{-1}\right]$ and the $\mathrm{v}_{\mathrm{p}}$-parameter for each voxel. The $\mathrm{K}_{\mathrm{i}}$ rate can be considered a measure of the permeability surface area product that indicates the leakage rate from the blood plasma to the brain tissue, and the $\mathrm{v}_{\mathrm{p}}$-parameter can be considered a measure of the blood plasma volume. Histograms of these parameters in the grey and white matter and hippocampus were created and corrected for noise (15), after which the mean $K_{i}$ and $v_{p}$ were calculated for each region. Due to the noise correction, mean $\mathrm{K}_{\mathrm{i}}$ per ROI generally has a lower value than the leakage rate of specific voxels (as depicted on overlays) as a relatively large number of voxels do not exhibit obvious leakage or leakage levels below the noise level.

\subsubsection{Statistics}

Among the cognitive scores, only Stroop interference was non-normally distributed. Difference score and current score of Stroop interference and the leakage rate and blood plasma volume parameters were cube-root transformed to obtain normal distributions. WMH volume and hippocampal volume were divided by intracranial volume to correct for head size (60) and log-transformed to obtain normal distributions.

Multiple linear regression was performed, with cognitive decline as dependent variable and leakage rate as predictor, in turn for the white matter, grey matter and hippocampus separately, while correcting for age, sex and education. G*power software was used to calculate the required sample size (64). We wanted to be able to detect a medium effect size $\left(f^{2}=0.15(65)\right)$, with an overall significance level of $5 \%$ and power set at a conventional $80 \%$. Power analysis for multiple linear regression using four 
predictors (66) demonstrated that 55 participants would be sufficient, and we eventually included 57 participants, taking into account the possibility that a few participants might have had to be excluded in the post-processing phase. The regression models were tested for each cognitive domain, resulting in a total of four times, and results were therefore corrected for multiple comparisons using the Benjamini-Hochberg procedure (67), with four tests and a false discovery rate (FDR) of 5\%. Additional analyses were performed using the most recent measure of cognitive performance instead of cognitive decline, and using the blood plasma volume instead of the leakage rate.

Post-hoc analyses were conducted to investigate the influence of other common indicators of structural brain integrity, namely WMH volume, cortical thickness and hippocampal volume, and blood plasma volume. These variables were separately added to the regression analyses as potential confounders for the relation between BBB leakage and cognition. Also, the association between these brain health measures and cognitive decline was investigated, using multiple linear regression with cognitive decline as dependent variable and WMH volume, cortical thickness, hippocampal volume or blood plasma volume as predictor, correcting for age, sex and education.

All statistical analyses were performed with commercial software (SPSS, version 24.0, IBM Corp., Armonk, NY, USA).

\subsection{Results}

Multiple linear regression was used to investigate the association between BBB leakage rate (white matter, grey matter and hippocampus) and 12-year cognitive decline (shortterm and long-term memory function, processing speed and executive functioning), in a usual aging sample ( $\mathrm{n}=57$ see Table 3.1 for sample characteristics). 
Chapter 3

Table 3.1. Participant $(n=57)$ characteristics

\begin{tabular}{lll}
\hline & $\begin{array}{l}\text { Mean } \\
\text { (standard deviation) / percentage } / \text { median }\left(25^{\text {th }}-75^{\text {th }} \text { percentile }\right)\end{array}$ \\
\hline Age & $65.8(10.2)$ & \\
\% Male & 52.6 & $15.8 / 54.4 / 29.8$ \\
\% Level of education $^{\mathbf{a}}$ & $1 / 2 / 3$ & \\
MMSEb $^{\mathbf{b}}$ & $29.0(28.0-30.0)$ & $5.3 / 70.2 / 12.3 / 12.3$ \\
\% WMH Fazekas $^{\mathbf{c}}$ & $0 / 1 / 2 / 3$ & $19.3 / 50.9 / 24.6 / 5.3$ \\
\% GCA $^{\mathbf{d}}$ & $0 / 1 / 2 / 3$ & \\
\% MTA $^{\text {e }}$ & & $78.9 / 17.5 / 3.5 / 0.0 / 0.0$ \\
Right hemisphere $_{\text {Left hemisphere }}$ & $0 / 1 / 2 / 3 / 4$ & $78.9 / 19.3 / 1.8 / 0.0 / 0.0$
\end{tabular}

a: Level of education: 1 = at most primary or lower vocational education; 2 = secondary education; 3 = higher vocational or scientific education

b: Mini-Mental State Examination score (44) at 12-year follow-up: maximum score $=30$, cognitively normal $\geq 25$

c: Fazekas visual rating score of white matter hyperintensity load (56): $0=$ absent: none or a single punctuate WMH lesion; 1 = mild: multiple punctuate lesions; 2 = moderate: beginning of confluency of lesions; 3 = severe: large confluent lesions

d: Global cortical atrophy visual rating score (57): 0 = absent: normal volume/no ventricular enlargement; 1 = mild: opening of sulci/mild ventricular enlargement; 2 = moderate: volume loss of gyri/moderate ventricular enlargement; 3 = severe: 'knife blade' atrophy/severe ventricular enlargement

e: Medial temporal lobe atrophy visual rating score (58): 0 = absent: no atrophy; 1 = marginal: only widening of choroid fissure; 2 = mild: also widening of temporal horn of lateral ventricle; 3 = moderate: moderate loss of hippocampal volume; $4=$ severe: severe volume loss of hippocampus

\subsubsection{Cognitive decline}

A significant decrease in cognitive score was found for immediate recall $(p=.023)$, processing speed $(\mathrm{p}<.001)$ and interference $(\mathrm{p}<.001)$.

\subsubsection{The association between BBB leakage and cognitive decline}

Older age is significantly associated with higher BBB leakage in the white matter $(\beta=$ $.306, \mathrm{p}=.024)$ and grey matter $(\beta=.286, \mathrm{p}=.035)$, as can be read in more detail in Verheggen et al. (2020) (50). Upon investigating whether this higher leakage is also 
associated with more age-related cognitive decline, we found a significant relation between BBB leakage in the white and grey matter and decline in delayed recall (Table 3.2; Figure 3.1; Figure 3.2), with higher BBB leakage rates being associated with a larger decrease in delayed recall score, while correcting for age, sex and education (white matter: $\beta=.389, p=.006$; grey matter: $\beta=.287, p=.044$ ). Out of the four cognitive domains, leakage rate had the strongest regression coefficients with delayed recall (Table 3.2).

Table 3.2. Median and interquartile range (IQR) of the leakage rates and mean and standard deviation (SD) of cognitive decline in each cognitive domain, and the standardized regression coefficient $(\beta)$ between leakage rate and cognitive decline, corrected for age, sex and education

\begin{tabular}{|c|c|c|c|c|c|}
\hline & & & $\begin{array}{l}\text { White } \\
\text { matter }\end{array}$ & $\begin{array}{l}\text { Grey } \\
\text { matter }\end{array}$ & Hippocampus \\
\hline & & $\mathbf{K}_{\mathbf{i}}$ & 1.1 & 0.9 & 1.7 \\
\hline & & {$\left[10^{-6} \cdot \min ^{-1}\right]$} & $(0.5 ; 2.0)$ & $(0.4 ; 1.6)$ & $(0.4 ; 4.2)$ \\
\hline & & Median & & & \\
\hline & & $(\mathrm{IQR})$ & & & \\
\hline & Cognitive decline $^{a}$ & & & & \\
\hline & Mean (SD) & & & & \\
\hline Immediate recall & $2.4(6.6)^{*}$ & & $\beta=.200$ & $\beta=.083$ & $\beta=.056$ \\
\hline Delayed recall & $0.3(2.9)$ & & $\beta=.389^{*}$ & $\beta=.287^{*}$ & $\beta=.175$ \\
\hline Processing speed & $5.1(4.4)^{*}$ & & $\beta=.075$ & $\beta=.069$ & $\beta=.038$ \\
\hline Interference $^{b}$ & $13.5(11.0)^{*}$ & & $\beta=.176$ & $\beta=.150$ & $\beta=.054$ \\
\hline a: Score last MAAS & leasure - current sc & & & & \\
\hline b: reversed by mult & plying with -1 & & & & \\
\hline *: significant at $\mathrm{p}<$. & & & & & \\
\hline
\end{tabular}


Chapter 3

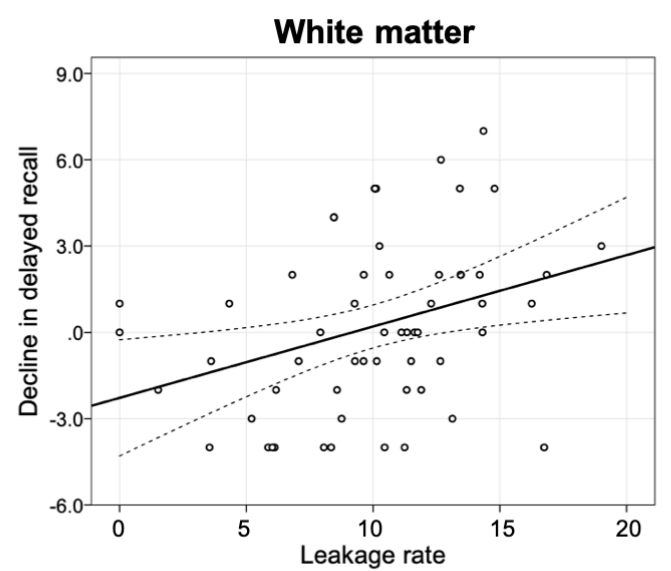

$\left(\sqrt[3]{\mathrm{K}_{\mathrm{i}}} \cdot 10^{3}\right)$

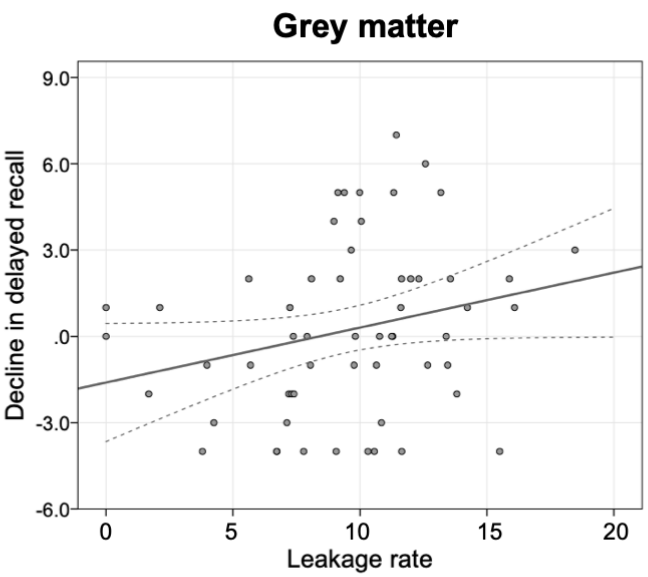

$\left(\sqrt[3]{\mathrm{K}_{\mathrm{i}}} \cdot 10^{3}\right)$

Figure 3.1. Regression analysis demonstrated that white and grey matter leakage rate is significantly associated with decline in delayed recall $(n=57)$.

After correction for multiple comparisons using the Benjamini-Hochberg procedure (67) (four tests, FDR = .05), only the association between decline in delayed recall and white matter leakage remained significant. 


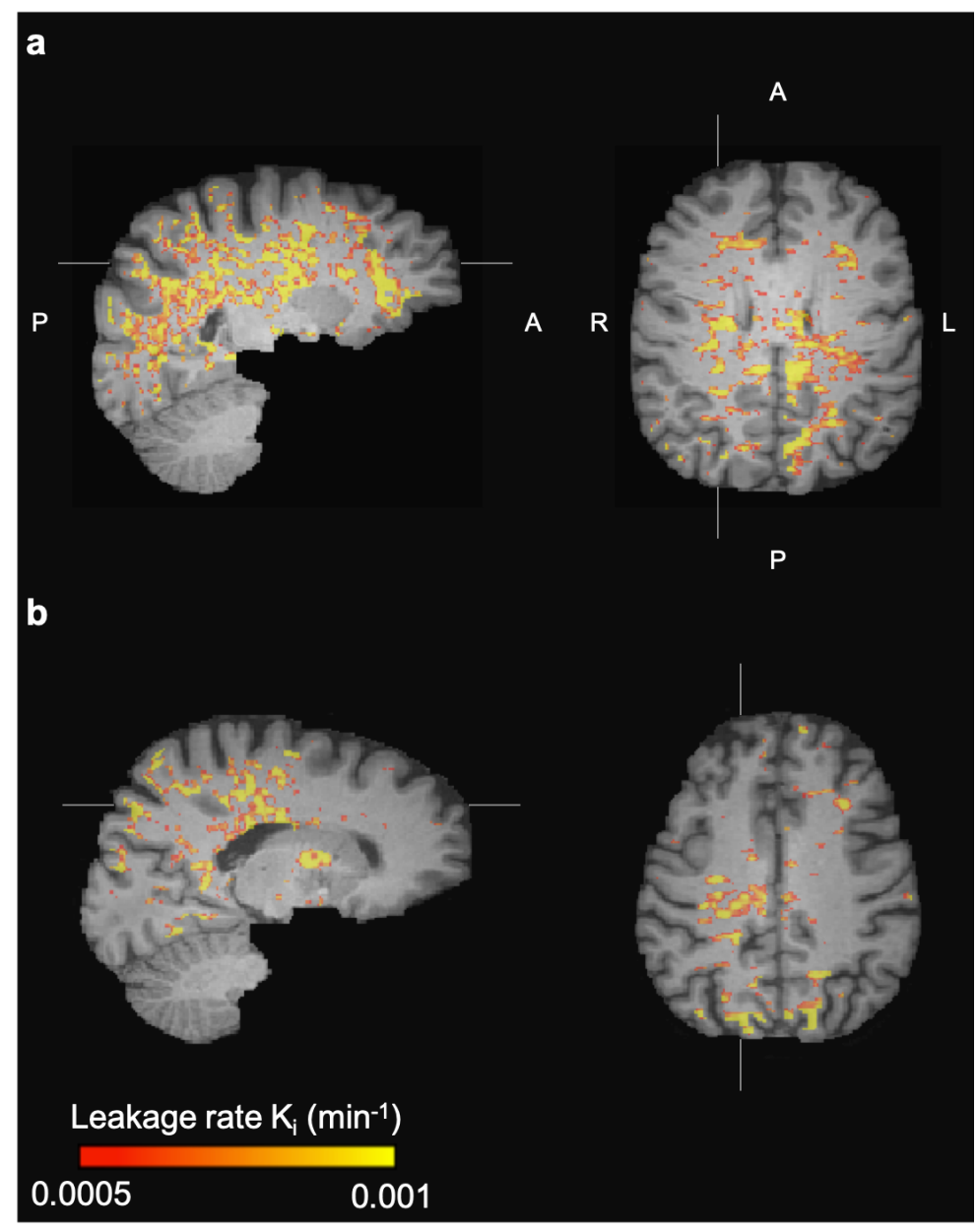

Figure 3.2. Leakage rate per voxel in the white matter of A: a woman (57 years) with a large decrease in the delayed recall score (remembering seven words less), and B: a man (62 years) with improved performance on the delayed recall test (remembering four words more).

Multiple linear regression with BBB leakage in the white matter as dependent variable and age as predictor, while correcting for sex, gave an unstandardized regression coefficient $(\beta)$ of $1.2 \cdot 10^{-4}$. This suggests that being 10 years older equals an increase of $1.2 \cdot 10^{-3}$ in the (cube-root transformed) white matter $\mathrm{K}_{\mathrm{i}}$ rate. The above reported regression with white matter $\mathrm{BBB}$ leakage as predictor of decline in delayed recall score (standardized $\beta=.39$ (Table 3.2), unstandardized $\beta=286$ ) can be used to calculate that this amount of leakage would correspond to a decline of 0.35 units $\left(286 \cdot\left(1.2 \cdot 10^{-3}\right)\right.$ ) on the VLT delayed recall score. Considering the baseline MAAS data from 1855 healthy participants (68), not taking BBB leakage into account, this decline in cognition would normally correspond to an increase of 4.7 years of age. Thus, over the 10 -year period, white matter BBB leakage alone is estimated to be associated with 4.7 years of cognitive aging. 


\subsubsection{The association between BBB leakage and current cognitive score}

No significant association was found between BBB leakage in the white or grey matter, or hippocampus, and the most recent cognitive score for each cognitive domain. However, the association between BBB leakage in the white and grey matter and delayed recall score approached significance (white matter: $\beta=-.259 \mathrm{p}=.055$; grey matter: $\beta=-$ $.264 \mathrm{p}=.050$ ), showing a trend towards more BBB leakage being associated with a worse performance on the delayed recall test.

\subsubsection{The association between blood plasma volume and cognitive decline} No significant association was found between blood plasma volume in the white matter, grey matter or hippocampus and decline in any of the cognitive domains (white matter: $\beta \geq-.171 \mathrm{p} \geq .19$; grey matter: $\beta \geq-.209 \mathrm{p} \geq .11$; hippocampus: $\beta \leq .139 \mathrm{p} \geq .33$ ).

Please note that the main analyses, i.e. BBB leakage and 12-year cognitive decline, was tested and corrected for multiple comparisons. No correction for multiple comparisons was applied to the analyses between BBB leakage and current cognitive score, or the analyses between blood plasma volume and cognitive decline, as these were additional analyses that yielded no significant results, and multiple comparisons correction, to check whether a significant result remains significant after correction, was not needed.

\subsubsection{The influence of WMH volume, cortical thickness, hippocampal volume and blood plasma volume}

Post-hoc analyses demonstrated that the association between BBB leakage in the white matter and decline in delayed recall remained significant after correction for WMH volume, cortical thickness, hippocampal volume or blood plasma volume. The association between grey matter BBB leakage and decline in delayed recall was just above significance when correcting for WMH volume $(\mathrm{p}=.061)$, cortical thickness $(\mathrm{p}=.064)$, hippocampal volume $(\mathrm{p}=.066)$ or blood plasma volume $(\mathrm{p}=.080)$. Results for the analyses with other ROIs or other cognitive domains did not change when adding WMH volume, cortical thickness, hippocampal volume or blood plasma volume to the regression analyses. None of these other MRI measures of structural brain integrity or blood plasma volume had a significant relation with cognitive decline. 


\subsection{Discussion}

In the current study, we investigated whether BBB disruption is related to usual agerelated cognitive decline. We found a significant association between BBB leakage and decrease in delayed recall score, which measures long-term episodic memory. Previous research has shown that decline in episodic memory is part of the normal aging process $(69,70)$. Both acquisition (the ability to encode newly learned information into memory) and retrieval (the ability to access newly learned information) decrease across the lifespan. The delayed recall score is considered a measure of retrieval and we demonstrated that BBB disruption in the white matter was significantly associated with impairment in retrieval. The current study estimated that over a period of 10 years, white matter BBB leakage alone would be associated with 4.7 years of cognitive aging. BBB disruption in the grey matter also showed an association with decline in retrieval, but this was less strong than in the white matter and no longer significant after correction for multiple comparisons.

The encoding of episodic events takes place in the hippocampus (71) first, and representations of these events are then stored in the cortex. For the retrieval function, both hippocampal and cortical processing is required with activation of a hippocampalcortical network $(71,72)$. Especially pathology in the white matter tracts can disrupt communication between the hippocampus and cortex and may impair network function. This corresponds to our finding that decline in retrieval is strongly related to BBB disruption throughout the cerebral white matter, rather than specifically in the hippocampus. For future studies, it would be interesting to investigate what white matter tracts are involved and in which white matter regions the association between BBB leakage and cognitive decline is most prominent.

A previous study found a significant association between BBB leakage and age and demonstrated that BBB leakage was significantly higher in individuals with mild cognitive impairment compared to normal controls (38). Remarkably, these relations were only found for BBB leakage in the hippocampus, and not for leakage in white matter or cortical grey matter structures. The authors propose that, in normal aging, BBB disruption possibly begins in the hippocampus and may subsequently contribute to agerelated cognitive impairment. However, while the current study demonstrated that BBB disruption was linked to usual age-related cognitive decline, we could only find this 
Chapter 3

association for BBB disruption in the cerebral white matter, but not in the hippocampus. Thus, we were not able to find support for the previous suggestion that early BBB leakage beginning in the hippocampus contributes to cognitive impairment in normal aging.

In a later study, the same group found higher BBB leakage in the hippocampus of individuals with early cognitive dysfunction compared to healthy controls (17). Possibly, the relation between $\mathrm{BBB}$ disruption in the hippocampus and cognitive performance is only present at a later stage of age-related cognitive deterioration. In another more recent study, they elaborate on this finding by demonstrating that cognitive impairment could especially be linked to BBB leakage in the hippocampus among APOE4 carriers (45). As the APOE4 allele greatly increases the risk of a neurodegenerative disorder, especially $\mathrm{AD}(40)$, this indicates that BBB disruption in the hippocampus might be more involved in pathological aging and might explain why the current study, focusing on successful aging, obtained a different result. However, another previous study, not only investigating normal controls, but also individuals with mild cognitive impairment and $\mathrm{AD}$, found no significant relation between $\mathrm{BBB}$ disruption in the hippocampus and cognitive performance (73). The authors suggest that, at a later stage of neurodegeneration, the effect of microvascular damage on cognitive performance might be negligible compared to the effect of neurodegenerative processes. This study also did not find a significant relation between BBB disruption in the white or grey matter and memory performance. The discrepancy with our results could be due to the use of longitudinal cognitive data in our study. When using only the most recent measure of cognitive performance, we did not find a significant relation either, though a trend approaching significance was visible. This could indicate that the influence of BBB disruption on cognitive performance is best visible in the long-term.

Performance on the cognitive tests is not only determined by cognitive aging, but also practice effects could influence the scores (74). Especially memory scores are known to be susceptible to practice $(75,76)$. Cognitive decline could result from a combination of more severe cognitive aging and less benefit from practice effects, and this combination can differ for each cognitive domain. Therefore, the current study did not aim to compare cognitive decline between the different domains. Instead, we investigated whether variation in a particular cognitive score was related to variation in leakage rate.

We did not find associations between BBB leakage and decline in immediate episodic memory as a measure of acquisition, processing speed, or the interference score 
as a measure of inhibition. As mentioned before, acquisition has been shown to decrease across the life span $(69,70)$. Also, processing speed decreases with age, starting in the third decade $(69,77)$, and research has shown that inhibition is compromised in normal aging, older adults $(78,79)$. Although each cognitive domain is thus known to be affected by age, only for decline in retrieval the association with BBB disruption reached significance. Previous imaging studies using diffusion tensor imaging (DTI) have suggested that usual age-related cognitive decline can at least partly be explained by white matter damage, with the greatest effect on cognitive functions that depend on communication between brain regions $(80,81)$. In a comparable sample of healthy, middle-aged to older individuals, no direct association could be found between white matter integrity, as measured with DTI, and processing speed or executive function (81). However, white matter integrity was significantly associated with working memory, possibly because working memory involves communication between the medial temporal and the frontal system $(80,82)$. Already before these findings, working memory had been linked to early normal aging, which was attributed to a disruption in balance between the two systems (83). As mentioned above, retrieval also involves communication between medial temporal lobe structures and distributed cortical areas $(71,72)$, so this cognitive function could be most vulnerable to usual age-related decline, which would explain why we only found a significant relation with decline in retrieval.

After correction for WMH volume, cortical thickness, hippocampal volume or blood plasma volume, BBB disruption in the white matter remained significantly associated with decline in retrieval. Thus, the relation between BBB disruption and usual age-related cognitive decline could not be explained by other physiological aging phenomena. BBB leakage being significantly associated with cognitive decline even after correction for blood plasma volume also indicates that the $\mathrm{K}^{\text {trans }}$ value is not simply dependent upon capillary density. No extensive white matter lesion load, cortical thinning or hippocampal volume loss was present in the current study, as can be seen in the overall low Fazekas, GCA and MTA ratings (Table 3.1), and these variables were not associated with cognitive aging. Also, while decreased vascularization has been associated with cognitive decline $(84,85)$, our study does not find any association between the blood plasma volume and cognition. However, BBB disruption was already associated with usual age-related cognitive decline at this stage, which supports the 
Chapter 3

notion that BBB disruption is an early event in cognitive aging, that might possibly affect brain function before these other processes.

To confirm our findings, longitudinal data should be collected to investigate whether BBB leakage increases as a person ages and whether this increase is paired with a change in cognitive function. Not only will a within-subject longitudinal design have more power as the results are no longer influenced by between-subjects variation, it will also allow us to comment on causality and uncover whether BBB disruption precedes cognitive changes and other brain pathologies. With BBB leakage rate only being obtained at the end of the 12-year interval, one cannot make inferences about causal relations, as persons with stronger BBB disruption and cognitive decline may have similar underlying origins. Neuroinflammation, for example, seems to form a vicious cycle with BBB disruption (86). Defensins penetrate the BBB and trigger the release of inflammatory cytokines, which leads to BBB disruption and consequently access of defensins, T-cells and inflammatory mediators into the brain, further promoting neuroinflammation. Another example is cerebral small vessel disease (cSVD). cSVD burden was demonstrated to correlate with both stronger BBB disruption and smaller blood plasma volume (87). Just like BBB disruption, neuroinflammation and cSVD have both been implicated in neurodegeneration and cognitive impairment $(86,88,89)$, and these processes may all be interacting with one another. It would have been preferable to measure BBB leakage at the moment of the first cognitive measure, so we would have been able to investigate whether baseline BBB disruption predicts future cognitive decline. However, 12 years ago, imaging techniques were not yet sensitive enough for the subtle leakage values in normal aging, which rendered the current design the best alternative. Moreover, BBB leakage needs to be MRI detectable at these younger ages in similarly healthy persons.

Another point of consideration for this study was the difficulty in identifying usual age-related cognitive decline. Participants recruited from MAAS have been participating in research for over 25 years, and may represent an exceptionally healthy subgroup, who are likely to perform above-average for their respective age groups. Cognitive decline in normal aging is subtle, especially in a sample this healthy. The standard cognitive tests, especially the memory scores, can lack sensitivity due to practice effects $(75,76)$, even in the current study with cognitive decline being calculated over a 12-year interval. To minimize these effects, different versions of the VLT were used at the different time 
points (but fixed per time point), which have been demonstrated to be equivalent (90). Moreover, this study benefitted from the use of the cognitive MAAS data (51). Initial practice has the strongest effect (75), and we were able to use the third MAAS measure as our baseline to minimize practice effects.

\subsection{Conclusion}

BBB disruption throughout the cerebral white matter was associated with decline in retrieval in normal aging. BBB breakdown being related to early cognitive decline supports the notion that BBB disruption could be a trigger of neuropathology eventually leading to neurodegeneration, and suggests that it could be a contributing mechanism to cognitive aging. Future research should investigate the pathological sequence of events by using longitudinal imaging data. Moreover, cardiovascular risk and life-style factors could be relevant to study possible mitigation of the observed effect. Also, recent studies suggest that restoring $\mathrm{NAD}^{+}$levels can protect $\mathrm{BBB}$ integrity (91-93), and this effort might become relevant for the maintenance of cognitive function.

\subsection{Acknowledgements}

The authors would like to acknowledge the participants for their contribution to scientific research, Walter M. Palm for neuroradiological assistance, Jos M.G.M. Slenter, Jan H. Jungerius and Ed H.B.M. Gronenschild for ongoing software, hardware and network support.

\subsection{Declaration of interest}

The authors declare that they have no competing interests.

\subsection{Ethical approval and consent}

The study was approved beforehand by the medical ethics review committee of Maastricht University Medical Center (approval number: METC163050). All participants provided written informed consent beforehand regarding participation and publication of the data.

\subsection{References}

1. He W, Goodkind D, Kowal PR. An aging world: 2015. 2016. 


\section{Chapter 3}

2. Rowe JW, Kahn RL. Successful aging. The gerontologist. 1997;37(4):433-40.

3. Glisky EL. Changes in cognitive function in human aging. Brain aging: models, methods, and mechanisms. 2007:3-20.

4. Rapp PR, Amaral DG. Individual differences in the cognitive and neurobiological consequences of normal aging. Trends in neurosciences. 1992;15(9):340-5.

5. Cabeza R, Albert M, Belleville S, Craik FI, Duarte A, Grady CL, et al. Maintenance, reserve and compensation: the cognitive neuroscience of healthy ageing. Nature Reviews Neuroscience. 2018;19(11):701-10.

6. Bishop NA, Lu T, Yankner BA. Neural mechanisms of ageing and cognitive decline. Nature. 2010;464(7288):529-35.

7. Camici GG, Liberale L. Aging: the next cardiovascular disease? European Heart Journal. 2017;38(21):1621-3.

8. Yang T, Sun Y, Lu Z, Leak RK, Zhang F. The impact of cerebrovascular aging on vascular cognitive impairment and dementia. Ageing research reviews. 2017;34:15-29.

9. Choi JY, Morris JC, Hsu CY. Aging and cerebrovascular disease. Neurologic clinics. 1998;16(3):687711.

10. Marchant NL, Reed BR, DeCarli CS, Madison CM, Weiner MW, Chui HC, et al. Cerebrovascular disease, beta-amyloid, and cognition in aging. Neurobiology of aging. 2012;33(5):1006. e25-. e36.

11. Mooradian AD. Potential mechanisms of the age-related changes in the blood-brain barrier. Neurobiology of Aging. 1994;15(6):751-5.

12. Farrall AJ, Wardlaw JM. Blood-brain barrier: ageing and microvascular disease-systematic review and meta-analysis. Neurobiology of aging. 2009;30(3):337-52.

13. Varatharaj A, Galea I. The blood-brain barrier in systemic inflammation. Brain, Behavior, and Immunity. 2017;60:1-12.

14. Zenaro E, Piacentino G, Constantin G. The blood-brain barrier in Alzheimer's disease. Neurobiology of Disease. 2017;107:41-56.

15. van de Haar HJ, Burgmans S, Jansen JF, van Osch MJ, van Buchem MA, Muller M, et al. Blood-brain barrier leakage in patients with early Alzheimer disease. Radiology. 2016;281(2):527-35.

16. Wang H, Golob EJ, Su M-Y. Vascular volume and blood-brain barrier permeability measured by dynamic contrast enhanced MRI in hippocampus and cerebellum of patients with MCI and normal controls. Journal of Magnetic Resonance Imaging. 2006;24(3):695-700.

17. Nation DA, Sweeney MD, Montagne A, Sagare AP, D’Orazio LM, Pachicano M, et al. Blood-brain barrier breakdown is an early biomarker of human cognitive dysfunction. Nature Medicine. 2019.

18. Zlokovic BV. Neurovascular pathways to neurodegeneration in Alzheimer\&\#39;s disease and other disorders. Nature Reviews Neuroscience. 2011;12:723.

19. Wilhelm I, Nyúl-Tóth Á, Suciu M, Hermenean A, Krizbai IA. Heterogeneity of the blood-brain barrier. Tissue Barriers. 2016;4(1):e1143544.

20. Buxton RB, Uludağ K, Dubowitz DJ, Liu TT. Modeling the hemodynamic response to brain activation. Neuroimage. 2004;23:S220-S33. 
21. Guo S, Lo EH. Dysfunctional cell-cell signaling in the neurovascular unit as a paradigm for central nervous system disease. Stroke. 2009;40(3_suppl_1):S4-S7.

22. Zhao Z, Nelson AR, Betsholtz C, Zlokovic BV. Establishment and dysfunction of the blood-brain barrier. Cell. 2015;163(5):1064-78.

23. Pardridge WM. Blood-brain barrier endogenous transporters as therapeutic targets: a new model for small molecule CNS drug discovery. Expert opinion on therapeutic targets. 2015;19(8):1059-72.

24. Montagne A, Zhao Z, Zlokovic BV. Alzheimer's disease: A matter of blood-brain barrier dysfunction? Journal of Experimental Medicine. 2017;214(11):3151-69.

25. Zlokovic BV. The blood-brain barrier in health and chronic neurodegenerative disorders. Neuron. 2008;57(2):178-201.

26. Wardlaw J, Sandercock P, Dennis M, Starr J. Is breakdown of the blood-brain barrier responsible for lacunar stroke, leukoaraiosis, and dementia? Stroke. 2003;34(3):806-12.

27. Zlokovic BV. Neurovascular mechanisms of Alzheimer's neurodegeneration. Trends in Neurosciences. 2005;28(4):202-8.

28. Kisler K, Nelson AR, Montagne A, Zlokovic BV. Cerebral blood flow regulation and neurovascular dysfunction in Alzheimer disease. Nature Reviews Neuroscience. 2017;18:419.

29. Iadecola C. The Neurovascular Unit Coming of Age: A Journey through Neurovascular Coupling in Health and Disease. Neuron. 2017;96(1):17-42.

30. Csiszar A, Tarantini S, Yabluchanskiy A, Balasubramanian P, Kiss T, Farkas E, et al. Role of endothelial NAD+ deficiency in age-related vascular dysfunction. American Journal of Physiology-Heartand Circulatory Physiology. 2019;316(6):H1253-H66.

31. Raja R, Rosenberg GA, Caprihan A. MRI measurements of Blood-Brain Barrier function in dementia: A review of recent studies. Neuropharmacology. 2018;134:259-71.

32. Fernando MS, Simpson JE, Matthews F, Brayne C, Lewis CE, Barber R, et al. White matter lesions in an unselected cohort of the elderly. Stroke. 2006;37(6):1391-8.

33. Burgmans S, van de Haar HJ, Verhey FR, Backes WH. Amyloid- $\beta$ interacts with blood-brain barrier function in dementia: a systematic review. Journal of Alzheimer's Disease. 2013;35(4):859-73.

34. Iadecola C, Zhang F, Niwa K, Eckman C, Turner SK, Fischer E, et al. SOD1 rescues cerebral endothelial dysfunction in mice overexpressing amyloid precursor protein. Nature Neuroscience. 1999;2:157.

35. Niwa K, Kazama K, Younkin L, Younkin SG, Carlson GA, Iadecola C. Cerebrovascular autoregulation is profoundly impaired in mice overexpressing amyloid precursor protein. American Journal of PhysiologyHeart and Circulatory Physiology. 2002;283(1):H315-H23.

36. Niwa K, Kazama K, Younkin SG, Carlson GA, Iadecola C. Alterations in Cerebral Blood Flow and Glucose Utilization in Mice Overexpressing the Amyloid Precursor Protein. Neurobiology of Disease. 2002;9(1):61-8.

37. Iturria-Medina Y, Sotero RC, Toussaint PJ, Mateos-Pérez JM, Evans AC, The Alzheimer's Disease Neuroimaging I, et al. Early role of vascular dysregulation on late-onset Alzheimer's disease based on multifactorial data-driven analysis. Nature Communications. 2016;7:11934. 


\section{Chapter 3}

38. Montagne A, Barnes SR, Sweeney MD, Halliday MR, Sagare AP, Zhao Z, et al. Blood-brain barrier breakdown in the aging human hippocampus. Neuron. 2015;85(2):296-302.

39. Montagne A, Nation DA, Pa J, Sweeney MD, Toga AW, Zlokovic BV. Brain imaging of neurovascular dysfunction in Alzheimer's disease. Acta neuropathologica. 2016;131(5):687-707.

40. Yamazaki Y, Zhao N, Caulfield TR, Liu C-C, Bu G. Apolipoprotein E and Alzheimer disease: pathobiology and targeting strategies. Nature Reviews Neurology. 2019;15(9):501-18.

41. Bell RD, Winkler EA, Singh I, Sagare AP, Deane R, Wu Z, et al. Apolipoprotein E controls cerebrovascular integrity via cyclophilin A. Nature. 2012;485(7399):512-6.

42. Salloway S, Gur T, Berzin T, Tavares R, Zipser B, Correia S, et al. Effect of APOE genotype on microvascular basement membrane in Alzheimer's disease. J Neurol Sci. 2002;203-204:183-7.

43. Halliday MR, Rege SV, Ma Q, Zhao Z, Miller CA, Winkler EA, et al. Accelerated pericyte degeneration and blood-brain barrier breakdown in apolipoprotein E4 carriers with Alzheimer's disease. J Cereb Blood Flow Metab. 2016;36(1):216-27.

44. Folstein MF, Folstein SE, McHugh PR. "Mini-mental state": a practical method for grading the cognitive state of patients for the clinician. Journal of psychiatric research. 1975;12(3):189-98.

45. Montagne A, Nation DA, Sagare AP, Barisano G, Sweeney MD, Chakhoyan A, et al. APOE4 leads to blood-brain barrier dysfunction predicting cognitive decline. Nature. 2020;581(7806):71-6.

46. Larsson HB, Courivaud F, Rostrup E, Hansen AE. Measurement of brain perfusion, blood volume, and blood-brain barrier permeability, using dynamic contrast-enhanced T1-weighted MRI at 3 tesla. Magnetic Resonance in Medicine: An Official Journal of the International Society for Magnetic Resonance in Medicine. 2009;62(5):1270-81.

47. van de Haar HJ, Burgmans S, Hofman PA, Verhey FR, Jansen JF, Backes WH. Blood-brain barrier impairment in dementia: current and future in vivo assessments. Neuroscience \& Biobehavioral Reviews. 2014.

48. Zhang CE, Wong SM, van de Haar HJ, Staals J, Jansen JF, Jeukens CR, et al. Blood-brain barrier leakage is more widespread in patients with cerebral small vessel disease. Neurology. 2017;88(5):426-32. 49. Pantoni L. Cerebral small vessel disease: from pathogenesis and clinical characteristics to therapeutic challenges. The Lancet Neurology. 2010;9(7):689-701.

50. Verheggen IC, de Jong JJ, van Boxtel MP, Gronenschild EH, Palm WM, Postma AA, et al. Increase in blood-brain barrier leakage in healthy, older adults. GeroScience. 2020:1-11.

51. Jolles J, Houx P, Van Boxtel M, Ponds R. Maastricht aging study: Determinants of cognitive aging: Neuropsych Publishers Maastricht; 1995.

52. Gélinas I, Gauthier L, McIntyre M, Gauthier S. Development of a functional measure for persons with Alzheimer's disease: the disability assessment for dementia. American Journal of Occupational Therapy. 1999;53(5):471-81.

53. Rey A. L'examen clinique en psychologie. 1958.

54. Natu M, Agarwal A. Digit letter substitution test (DLST) as an alternative to digit symbol substitution test (DSST). Human Psychopharmacology: Clinical and Experimental. 1995;10(4):339-43.

55. Stroop JR. Studies of interference in serial verbal reactions. Journal of experimental psychology. 1935;18(6):643. 
56. Fazekas F, Kleinert R, Offenbacher H, Schmidt R, Kleinert G, Payer F, et al. Pathologic correlates of incidental MRI white matter signal hyperintensities. Neurology. 1993;43(9):1683-.

57. Pasquier F, Leys D, Weerts JG, Mounier-Vehier F, Barkhof F, Scheltens P. Inter-and intraobserver reproducibility of cerebral atrophy assessment on MRI scans with hemispheric infarcts. European neurology. 1996;36(5):268-72.

58. Scheltens P, Leys D, Barkhof F, Huglo D, Weinstein H, Vermersch P, et al. Atrophy of medial temporal lobes on MRI in" probable" Alzheimer's disease and normal ageing: diagnostic value and neuropsychological correlates. Journal of Neurology, Neurosurgery \& Psychiatry. 1992;55(10):967-72.

59. Jacobs HI, Clerx L, Gronenschild EH, Aalten P, Verhey FR. White matter hyperintensities are positively associated with cortical thickness in Alzheimer's disease. Journal of Alzheimer's Disease. 2014;39(2):409-22.

60. Schwarz CG, Gunter JL, Wiste HJ, Przybelski SA, Weigand SD, Ward CP, et al. A large-scale comparison of cortical thickness and volume methods for measuring Alzheimer's disease severity. NeuroImage: Clinical. 2016;11:802-12.

61. Fischl B, Sereno MI, Dale AM. Cortical Surface-Based Analysis: II: Inflation, Flattening, and a Surface-Based Coordinate System. NeuroImage. 1999;9(2):195-207.

62. van de Haar HJ, Jansen JF, van Osch MJ, van Buchem MA, Muller M, Wong SM, et al. Neurovascular unit impairment in early Alzheimer's disease measured with magnetic resonance imaging. Neurobiology of aging. 2016;45:190-6.

63. Cramer SP, Larsson HB. Accurate determination of blood-brain barrier permeability using dynamic contrast-enhanced T1-weighted MRI: a simulation and in vivo study on healthy subjects and multiple sclerosis patients. Journal of Cerebral Blood Flow \& Metabolism. 2014;34(10):1655-65.

64. Faul F, Erdfelder E, Lang A-G, Buchner A. G* Power 3: A flexible statistical power analysis program for the social, behavioral, and biomedical sciences. Behavior research methods. 2007;39(2):175-91.

65. Cohen J. A power primer. Psychological bulletin. 1992;112(1):155.

66. Faul F, Erdfelder E, Buchner A, Lang A-G. Statistical power analyses using G* Power 3.1: Tests for correlation and regression analyses. Behavior research methods. 2009;41(4):1149-60.

67. Benjamini Y, Hochberg Y. Controlling the false discovery rate: a practical and powerful approach to multiple testing. Journal of the Royal statistical society: series B (Methodological). 1995;57(1):289-300. 68. Van Der Elst W, Van Boxtel MP, Van Breukelen GJ, Jolles J. Rey's verbal learning test: normative data for 1855 healthy participants aged 24-81 years and the influence of age, sex, education, and mode of presentation. Journal of the International Neuropsychological Society. 2005;11(03):290-302.

69. Harada CN, Love MCN, Triebel KL. Normal cognitive aging. Clinics in geriatric medicine. 2013;29(4):737-52.

70. Rönnlund M, Nyberg L, Bäckman L, Nilsson L-G. Stability, Growth, and Decline in Adult Life Span Development of Declarative Memory: Cross-Sectional and Longitudinal Data From a Population-Based Study. Psychology and Aging. 2005;20(1):3-18.

71. Eichenbaum H. Hippocampus: cognitive processes and neural representations that underlie declarative memory. Neuron. 2004;44(1):109-20. 


\section{Chapter 3}

72. Eichenbaum H. A cortical-hippocampal system for declarative memory. Nature Reviews Neuroscience. 2000;1(1):41-50.

73. Freeze WM, Jacobs HI, de Jong JJ, Verheggen IC, Gronenschild EH, Palm WM, et al. White matter hyperintensities mediate the association between blood-brain barrier leakage and information processing speed. Neurobiology of aging. 2020;85:113-22.

74. Salthouse TA. When does age-related cognitive decline begin? Neurobiology of aging. 2009;30(4):507-14.

75. Machulda MM, Pankratz VS, Christianson TJ, Ivnik RJ, Mielke MM, Roberts RO, et al. Practice effects and longitudinal cognitive change in normal aging vs. incident mild cognitive impairment and dementia in the Mayo Clinic Study of Aging. The Clinical Neuropsychologist. 2013;27(8):1247-64.

76. McCaffrey RJ, Ortega A, Haase RF. Effects of repeated neuropsychological assessments. Archives of Clinical Neuropsychology. 1993;8(6):519-24.

77. Salthouse TA. Selective review of cognitive aging. Journal of the International neuropsychological Society. 2010;16(5):754-60.

78. Wecker NS, Kramer JH, Wisniewski A, Delis DC, Kaplan E. Age effects on executive ability. Neuropsychology. 2000;14(3):409.

79. Daigneault S, Braun CM, Whitaker HA. Early effects of normal aging on perseverative and nonperseverative prefrontal measures. Developmental Neuropsychology. 1992;8(1):99-114.

80. Charlton RA, Barrick TR, Lawes INC, Markus HS, Morris RG. White matter pathways associated with working memory in normal aging. Cortex. 2010;46(4):474-89.

81. Charlton RA, Barrick TR, McIntyre DJ, Shen Y, O'Sullivan M, Howe FA, et al. White matter damage on diffusion tensor imaging correlates with age-related cognitive decline. Neurology. 2006;66(2):217-22.

82. Constantinidis C, Procyk E. The primate working memory networks. Cognitive, Affective, \& Behavioral Neuroscience. 2004;4(4):444-65.

83. Daigneault S, Braun CM. Working memory and the self-ordered pointing task: Further evidence of early prefrontal decline in normal aging. Journal of clinical and Experimental Neuropsychology. 1993;15(6):881-95.

84. Lista I, Sorrentino G. Biological mechanisms of physical activity in preventing cognitive decline. Cellular and molecular neurobiology. 2010;30(4):493-503.

85. Ajmani R, Metter E, Jaykumar R, Ingram D, Spangler E, Abugo 0, et al. Hemodynamic changes during aging associated with cerebral blood flow and impaired cognitive function. Neurobiology of aging. 2000;21(2):257-69.

86. Kazakos EI, Kountouras J, Polyzos SA, Deretzi G. Novel aspects of defensins' involvement in virusinduced autoimmunity in the central nervous system. Medical Hypotheses. 2017;102(Supplement C):33-6. 87. Li Y, Li M, Zuo L, Shi Q, Qin W, Yang L, et al. Compromised Blood-Brain Barrier Integrity Is Associated With Total Magnetic Resonance Imaging Burden of Cerebral Small Vessel Disease. Front Neurol. 2018;9:221.

88. Shlosberg D, Benifla M, Kaufer D, Friedman A. Blood-brain barrier breakdown as a therapeutic target in traumatic brain injury. Nature Reviews Neurology. 2010;6(7):393-403. 
89. Debette S, Beiser A, DeCarli C, Au R, Himali JJ, Kelly-Hayes M, et al. Association of MRI markers of vascular brain injury with incident stroke, mild cognitive impairment, dementia, and mortality: the Framingham Offspring Study. Stroke. 2010;41(4):600-6.

90. Shapiro DM, Harrison DW. Alternate forms of the AVLT: a procedure and test of form equivalency. Archives of Clinical Neuropsychology. 1990;5(4):405-10.

91. Mills KF, Yoshida S, Stein LR, Grozio A, Kubota S, Sasaki Y, et al. Long-term administration of nicotinamide mononucleotide mitigates age-associated physiological decline in mice. Cell metabolism. 2016;24(6):795-806.

92. Zhang H, Ryu D, Wu Y, Gariani K, Wang X, Luan P, et al. NAD+ repletion improves mitochondrial and stem cell function and enhances life span in mice. Science. 2016;352(6292):1436-43.

93. Yoshino J, Baur JA, Imai S-i. NAD+ intermediates: the biology and therapeutic potential of NMN and NR. Cell metabolism. 2018;27(3):513-28. 



\section{Chapter 4}

Permeability of the windows of the brain:

Feasibility of dynamic contrast-enhanced

\section{MRI of the circumventricular organs}

Fluids Barriers CNS 17, 66 (2020). https://doi.org/10.1186/s12987-020-00228-x

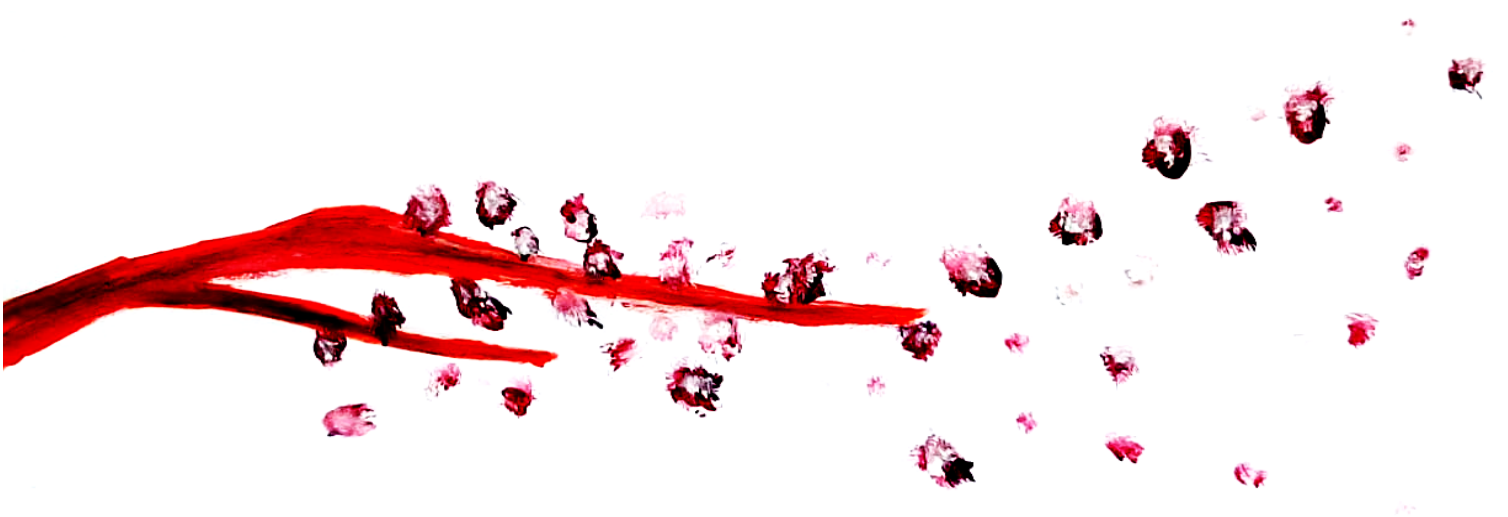


Chapter 4

\subsection{Abstract}

\section{Background}

Circumventricular organs (CVOs) are small structures without a blood-brain barrier surrounding the brain ventricles that serve homeostatic functions and facilitate communication between the blood, cerebrospinal fluid and brain. Secretory CVOs release peptides and sensory CVOs regulate signal transmission. However, pathogens may enter the brain through the CVOs and trigger neuroinflammation and neurodegeneration. We investigated the feasibility of dynamic contrast-enhanced (DCE) MRI to assess the CVO permeability characteristics in vivo, and expected significant contrast uptake in these regions, due to blood-brain barrier absence.

\section{Methods}

Twenty healthy, middle-aged to older males underwent brain DCE MRI. Pharmacokinetic modelling was applied to contrast concentration time-courses of CVOs, and in reference to white and grey matter. We investigated whether a significant and positive transfer from blood to brain could be measured in the CVOs, and whether this differed between secretory and sensory CVOs or from normal-appearing brain matter.

\section{Results}

In both the secretory and sensory CVOs, the transfer constants were significantly positive, and all secretory CVOs had significantly higher transfer than each sensory CVO. The transfer constants in both the secretory and sensory CVOs were higher than in the white and grey matter.

\section{Conclusions}

Current measurements confirm the often-held assumption of highly permeable CVOs, of which the secretory types have the strongest blood-to-brain transfer. The current study suggests that DCE MRI could be a promising technique to further assess the function of the CVOs and how pathogens can potentially enter the brain via these structures.

Netherlands Trial Register number: NL6358, date of registration: 2017-03-24

\section{Keywords}

Circumventricular organs, dynamic contrast-enhanced magnetic resonance imaging, permeability, pharmacokinetic modelling 


\subsection{Introduction}

\subsubsection{The circumventricular organs}

The circumventricular organs (CVOs) are structures located adjacent to the third and fourth ventricles of the brain (1-3). An important characteristic of these structures is that they have extensive and highly permeable capillaries that lack a blood-brain barrier (BBB). The vessels in the CVOs branch into a network of fenestrated capillaries with loosely connected astrocytic endfeet (Figure 4.1). As a consequence, substances can travel freely between the blood and the CVO tissue, making neurons more susceptible to peripheral signals (4). The CVOs contain ependymal cells called tanycytes that have apical processes that extend into the cerebrospinal fluid (CSF) to monitor CSF composition and distal processes that contact the fenestrated capillaries to control the exchange of substances between the blood and CSF $(1,4-6)$. The tanycytes contain tight junction proteins (Figure 4.1). Although a barrier between the vasculature and brain tissue is missing, these tight junctions form a barrier guarding the ventricular components and controlling the diffusion of blood-borne substances to the CSF (7). The CVOs are considered as points of communication between the blood, CSF and brain parenchyma and, in general, serve to maintain homeostasis $(6,8)$.

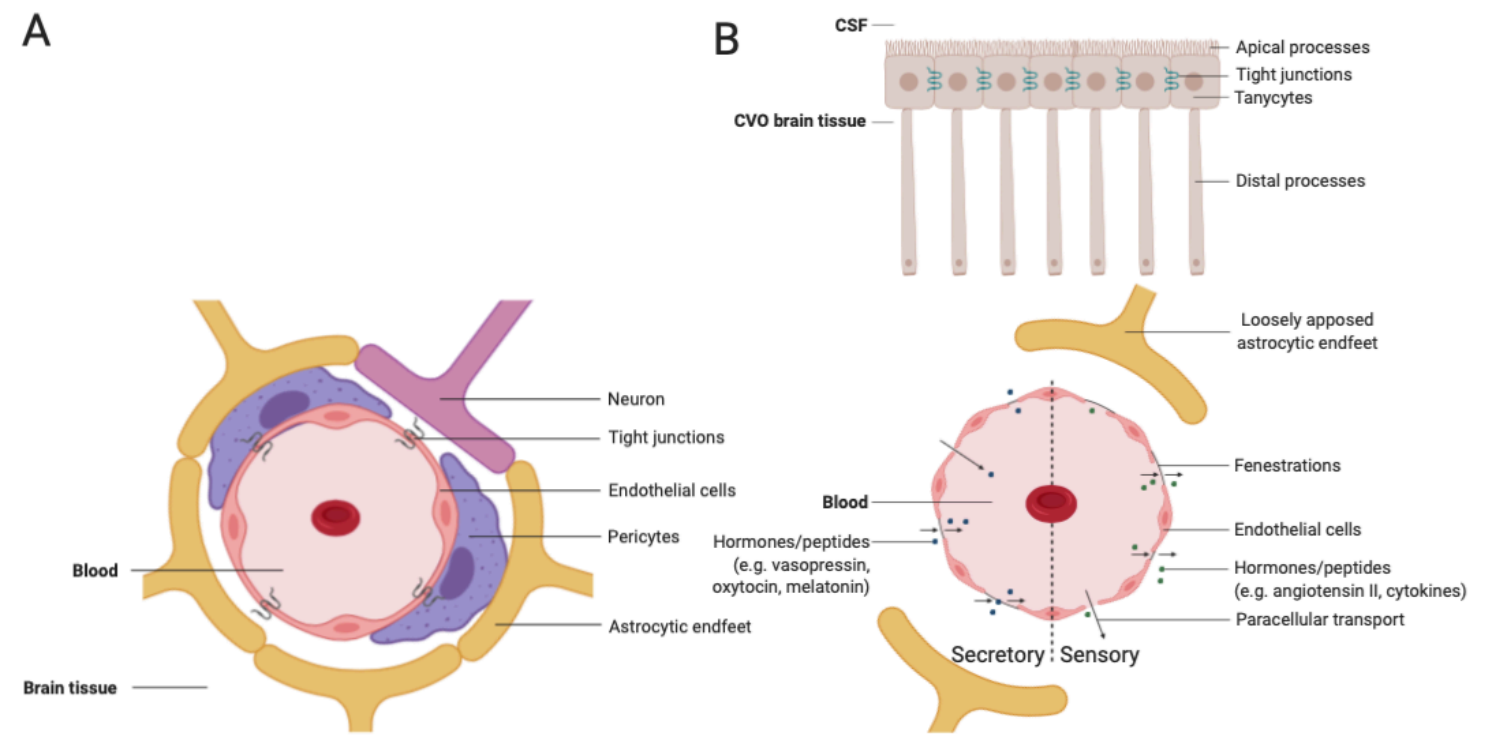

Figure 4.1. A: cross-section of a blood vessel with a normal blood-brain barrier. B: circumventricular organ with a cross-section of a capillary with a fenestrated endothelium, with on the left half the secretory and on the right half the sensory type. Tanycytes monitor cerebrospinal fluid composition and pass this information to the circumventricular organ. Figure created with BioRender.com. 
Chapter 4

\subsubsection{Functions of the circumventricular organs}

The CVOs can be divided in two groups: secretory and sensory types (Figure 4.1). The secretory CVOs include the neurohypophysis (NH), median eminence (ME) and pineal gland (PG) $(1,5)$. The secretory CVOs are involved in hormone and peptide secretion, neurochemical transport and chemoreception (5) (Table 4.1). The sensory CVOs include the subfornical organ (SFO), organum vasculosum of the lamina terminalis (OVLT) and area postrema (AP) (5).

The sensory CVOs can sample molecules from the blood and brain interstitial fluid and pass this information on to other brain structures. They have connections with major neural effector centers for most autonomic functions and have important roles in sodium and water balance, cardiovascular regulation, energy metabolism and immunomodulation $(1,9)$ (Table 4.1). The sensory CVOs allow communication between the endocrine, immune and central nervous system and thereby contribute to body fluid homeostasis (4). For example, angiotensin, a hormone regulating vasoconstriction, arterial pressure and cardiovascular function, acts through signalling into the sensory CVOs (10). Via their action on CVOs, pharmacotherapy such as angiotensin blockers can decrease blood pressure and cardiovascular morbidity (11).

The subcommissural organ (SCO) is sometimes considered to be a CVO, due to its extensive communication with the CSF (2). However, the SCO does not have a large network of fenestrated capillaries (5), which is an essential feature of the CVOs, and therefore, we did not include the SCO as a CVO.

The CVOs also play a role in inflammation as they can be entry points for inflammatory cells and are involved in cytokine secretion $(12,13)$. The immune system communicates with the brain to activate the hypothalamic-pituitary-adrenal-axis (HPAaxis) (14). This communication involves activation of the CVOs, which subsequently secrete pro-inflammatory cytokines. Many disorders, such as depression, schizophrenia and Alzheimer's disease (AD), are associated with disruption of the HPA-axis (15). Investigating the connection between the CVOs and HPA-circuits may lead to the detection of abnormalities at early stages of disease (4).

Studies in sheep have demonstrated that blood-borne prions can enter the central nervous system (CNS) via the CVOs $(16,17)$. Prion accumulation is linked to chronic inflammation in prion disease. Prion accumulation occurs in the CVOs, from where the infection can spread to other brain areas. When pathogens enter through the CVOs, they 
can activate a cytokine-transcription cascade which produces prostaglandins, subsequently leading to more widespread BBB disruption and CNS pathology (4).

The CVOs have thus been linked to neuroinflammation and characteristics of neurodegenerative disorders and may be involved in neurotoxic protein accumulation. More knowledge on the CVOs could possibly help in the detection of abnormalities at early stages of disease, as they may be the first place for neurotoxins to accumulate, or offer a new pharmaceutical target not hampered by the BBB.

\subsubsection{Imaging techniques for the circumventricular organs}

Finding a feasible method to visualize the CVOs is an important first step in gaining more in vivo knowledge on these rather small structures and their role in disease etiology. Since the CVOs do not have a BBB, a contrast agent is expected to transfer rapidly from the blood plasma into the brain tissue. Previous studies have used post-gadolinium enhancement brain MRI to assess CVO visibility $(5,18)$. In the current study, we use brain images obtained with a dynamic contrast-enhanced (DCE) MRI technique, which can detect dynamic concentration changes of intravenously administered gadolinium contrast-agent, to investigate whether it is feasible to assess the permeability properties of the CVOs. This DCE MRI technique uses a dual-time resolution protocol, which makes our measurements susceptible to both the early rapid temporal changes capturing the vascular component and the later slower features to capture contrast agent extravasation (i.e. permeability) (19).

\subsubsection{Research question}

Our main hypothesis states that significant and positive gadolinium-based contrast agent transfer will be detected in the CVO tissue, due to the absence of the BBB. Additionally, we investigated whether permeability in the secretory CVOs differed from that in the sensory CVOs, and whether permeability in the CVOs differed from the permeability measured in the normal-appearing brain matter. Moreover, cerebral blood flow, which influences blood-to-brain transfer $(20,21)$, is known to decrease during aging (22). Therefore, we investigated whether the contrast enhancement in the CVOs was agedependent. 
Table 4.1. Short overview of the characteristics of the circumventricular organs

\begin{tabular}{|c|c|c|c|c|c|c|}
\hline & Type & Location & Size $[\mathrm{mm}]$ & Primary function & Hormones & Ref. \\
\hline NH & Secretory & $\begin{array}{l}\text { Posterior part of the hypophysis } \\
\text { originating from the floor of the third } \\
\text { ventricle }\end{array}$ & $1.0-2.9$ & $\begin{array}{l}\text { Releasing hormones received from the } \\
\text { paraventricular and supraoptic nuclei }\end{array}$ & $\begin{array}{l}\text { Vasopressin } \\
\text { Oxytocin }\end{array}$ & $(2,23)$ \\
\hline ME & Secretory & $\begin{array}{l}\text { Extension of the floor of the third } \\
\text { ventricle }\end{array}$ & 0.3 & $\begin{array}{l}\text { Hypophysial portal system: transporting } \\
\text { hormones to the hypophysis }\end{array}$ & $\begin{array}{l}\text { Vasopressin } \\
\text { Oxytocin }\end{array}$ & $(2,24)$ \\
\hline PG & Secretory & Posterior wall of the third ventricle & 1.7 & Regulating circadian rhythms & Melatonin & $(2,6)$ \\
\hline OVLT & Sensory & Rostral wall of the third ventricle & 0.2 & $\begin{array}{l}\text { Water and sodium homeostasis and immune } \\
\text { response }\end{array}$ & $\begin{array}{l}\text { Angiotensin II } \\
\text { Cytokines }\end{array}$ & $(2,4,6)$ \\
\hline SFO & Sensory & $\begin{array}{l}\text { Inferior surface of the fornix/ } \\
\text { roof of the third ventricle }\end{array}$ & $0.3-0.6$ & Water and sodium homeostasis & Angiotensin II & $(2,6)$ \\
\hline AP & Sensory & Floor of the fourth ventricle & 0.5 & $\begin{array}{l}\text { Opening the central canal, cardiovascular and } \\
\text { respiratory regulation and controlling the } \\
\text { vomiting center }\end{array}$ & Substance P & $(2,4,6)$ \\
\hline
\end{tabular}

Abbreviations: $\mathrm{NH}$ = neurohypophysis; $\mathrm{ME}$ = median eminence; $\mathrm{PG}$ = pineal gland; $\mathrm{SFO}$ = subfornical organ; OVLT = organum vasculosum of the lamina terminalis; AP = area postrema. 


\subsection{Methods}

\subsubsection{Participants}

Recently, a follow-up on a subsample of participants of the Maastricht Aging Study (MAAS) (25) was conducted, in which 61 individuals who had shown no evidence of cognitive or functional decline were included (Mini-Mental State Examination score $\geq 25$; Disability Assessment for Dementia score > 90\%; no diagnosis of dementia, mild cognitive impairment or other psychiatric or neurological disorders; no structural brain abnormalities; no cognitive impairment due to substance abuse). All participants provided written informed consent before participation. For the current study, we used the imaging data of the 10 youngest (median age $=53.5$ years, age range $=47-56$ ) and the 10 oldest (median age $=73.5$ years, age range: 70 - 91) male participants (Supplement 1, Table 1.1.). Previous studies have demonstrated that the estrous cycle influences CVO activity (26-28), so only male individuals were included in this feasibility study.

\subsubsection{MRI acquisition}

Anatomical and DCE MRI data were acquired using a 3 Tesla MRI system (Achieva TX, Philips Healthcare, Best, the Netherlands) with a 32-channel head coil. The imaging protocol included a 3D T1-weighted inversion recovery fast gradient echo (repetition time (TR) of $8 \mathrm{~ms}$; inversion time (TI) of $800 \mathrm{~ms}$; echo time (TE) of $4 \mathrm{~ms}$; flip angle of $8^{\circ}$; $1 \mathrm{~mm}$ cubic voxel size) for anatomic reference; a 3D T2-weighted fluid attenuation inversion recovery (FLAIR) (TR/TI/TE of 4800/1650/290 ms; flip angle of $90^{\circ} ; 1 \mathrm{~mm}$ cubic voxel size) for localizing the CVOs; and a dual-time resolution dynamic contrastenhanced (DCE) MRI acquisition. The dual-time DCE MRI protocol consisted of two nested pulse sequences, a slow and a fast sequence with a saturation recovery preparation pulse, as described earlier (29). In short, the fast sequence used a short dynamic scan interval of $3.2 \mathrm{~s}$ during the steep signal changes in initial circulations of the contrast agent, while the slow sequence used a longer interval of $30.5 \mathrm{~s}$ during the later extravasation phase when the signal changes are much slower. Before contrast administration (pre-contrast), scans of both sequences were acquired. Subsequently, a bolus injection of gadolinium-based contrast agent was performed during the fast sequence (0.1 mmol/kg gadobutrol, Gadavist ${ }^{\circledR}$, Bayer AG, Leverkusen, Germany), intravenously in the antecubital vein (injection rate $3 \mathrm{~mL} / \mathrm{s}, 20 \mathrm{~mL}$ saline flush). The fast sequence consisted of 29 volumes (TR/TE/delay time (TD) 5.3/2.5/120 ms, voxel size 2 
Chapter 4

x 2 x $5 \mathrm{~mm}$ ) and the slow sequence consisted of 30 volumes (TR/TE/TD 5.6/2.5/120 ms, voxel size 1 x 1 x $2 \mathrm{~mm}$ ). To minimize partial volume, fold over, and inflow effects (of the sagittal sinus superior), an odd number of sagittal orientated slices, 11 for the fast sequence and 75 for the slow sequence, was acquired with the frequency-encoding direction in the craniocaudal direction. T1-mapping with variable delay time settings was performed prior to contrast administration and DCE imaging to enable the conversion of the contrast-enhanced tissue signal intensities to contrast agent concentrations (30).

\subsection{3. $\quad$ Brain regions of interest}

One researcher (I.C.M.V.) was trained by an experienced neuroradiologist (A.A.P.) in determining the locations of the CVOs on the basis of brain anatomy from the mid-sagittal anatomical FLAIR images. Recognizable anatomical identification marks were used to determine the location of each CVO (e.g. the hypophysis and mammillary body for the NH and ME; the anterior commissure for the OVLT; the hippocampus and fornix for the SFO; the fourth ventricle for the AP), and a limited number of voxels on this exact location was selected, which were saved as region-of-interest (ROI).
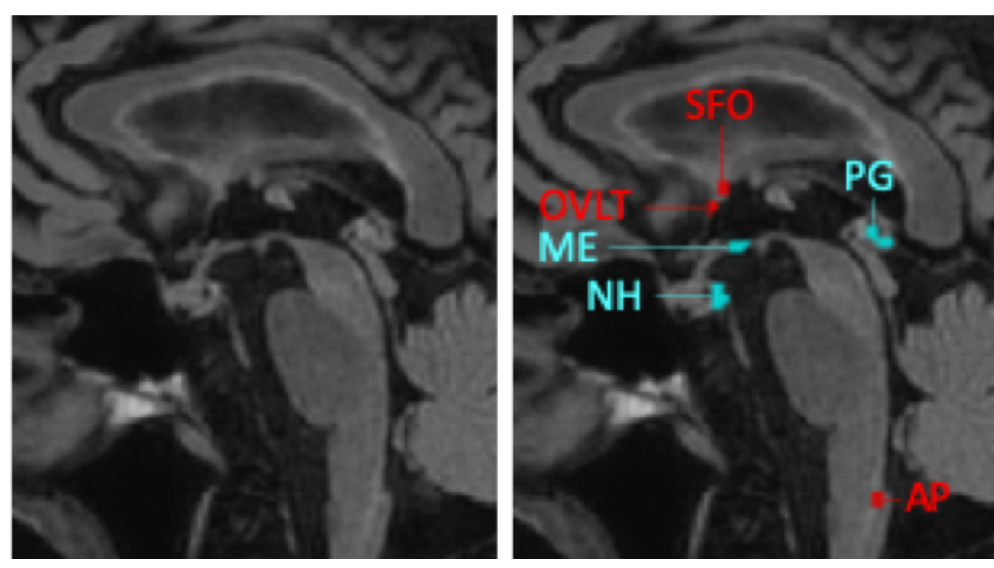

Figure 4.2. Example of a mid-sagittal anatomical FLAIR image and the regions-of-interest of the secretory (blue) and sensory (red) circumventricular organs.

Abbreviations: $N H=$ neurohypophysis, $M E=$ median eminence, $P G=$ pineal gland, $S F O=$ subfornical organ, OVLT = organum vasculosum of the lamina terminalis, $A P=$ area postrema .

Additionally, a ROI was placed in the neck muscle, to observe the temporal enhancement time curves for qualitative comparisons of the contrast agent distribution. As quantitative control, the white and grey matter regions were selected, which were segmented using 
automated software (FreeSurfer, version 6.0.0 (31)). The FreeSurfer segmentation was visually checked by one researcher (I.C.M.V.) with manual adjustments. From the segmented brain regions, the total grey matter (including cortical grey matter, deep grey matter (thalamus, caudate nucleus, putamen, pallidum, amygdala, and accumbens area), and hippocampus) and total white matter volume were extracted (31).

\subsubsection{Pre-processing}

The slow and fast dynamic series were motion corrected and spatially aligned using a linear registration procedure with six degrees of freedom (FLIRT, FMRIB's linear registration tool), with the average pre-contrast slow volume as reference. Next, the motion-corrected dynamic series, the FLAIR images and tissue masks, and $\mathrm{T}_{10}$-map were subsequently registered onto the participant's structural T1-weighted images.

Individual vascular input functions (VIFs) were extracted from manually (I.C.M.V.) selected voxels $(\geq 20)$ in the superior sagittal sinus $(32,33)$. Conversion of MRI signal enhancement to contrast agent concentration was performed differently for the VIF and tissue, and has been described earlier in detail (34). In short, the VIF signal-toconcentration conversion was implemented using in vitro data (diluted $\mathrm{MnCl}_{2}$ stock solution with different gadobutrol concentrations (1-40 mM), baseline T1 relaxation time of $1650 \mathrm{~ms}$, comparable to human blood), whereas the conversion to contrast agent concentration in tissue was performed assuming a linear relationship and a tissue relaxation time calculated from the $\mathrm{T}_{10}$-map. Representative contrast agent concentration maps pre- and post-contrast agent injection are shown in Figure 4.3. 


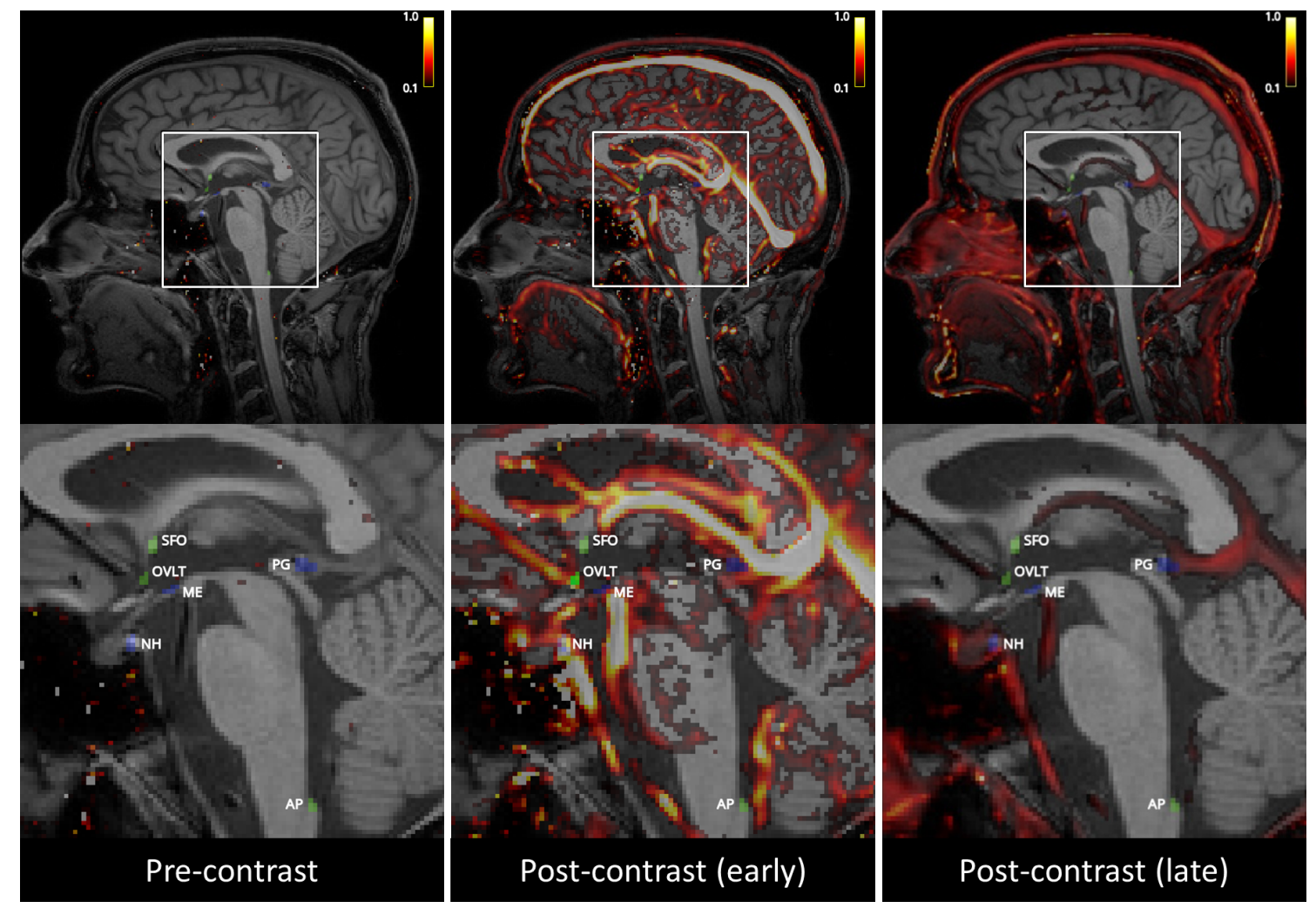

Figure 4.3. Representative contrast agent concentration maps [mM] showing the distribution of contrast agent before (left), shortly after (middle), and approximately 12 minutes after (right) contrast agent injection.

\subsection{5. $\quad$ Pharmacokinetic modelling}

The CVOs are the points of communication between the blood plasma and the brain tissue and their functionality involves extensive exchange with the blood circulation comprising influx and reflux of solutes $(4,6)$. To take both the influx and reflux into account, we applied the extended Tofts model (ETM) to the CVO data, which is a two-compartmental model with a blood compartment and an interstitial compartment with bidirectional transport between these compartments (35). The CVOs are expected have a high permeability due to the lack of a blood-brain barrier and thus a sufficiently high signalto-noise ratio. Therefore, the ROI averaged concentration-time data per CVO were fitted using the ETM as implemented in ROCKETSHIP (36) (fitting parameters for $\mathrm{K}^{\text {trans: }}$ starting value of $0.001 \mathrm{~min}^{-1}$; lower and upper bound value of -2 and $2 \mathrm{~min}^{-1}$; maximum number of iterations of 50; and a function tolerance of $10^{-12}$ ) to obtain $\mathrm{K}^{\text {trans }}\left[\mathrm{min}^{-1}\right.$, the transfer constant from blood plasma to extracellular, extravascular space as measure of permeability, $v_{p}[-]$, the volume fraction of blood plasma within a ROI as measure of perfusion, and $v_{e}[-]$, extravascular space volume fraction as a measure of uptake capacity 
and retention, for each CVO. The ETM provided sufficiently good fits for both secretory and sensory CVOs (Figure 4.4).

Next, to check whether the ETM sufficiently fitted the CVO data, each fit was visually checked (I.C.M.V.) and rated as 'good', 'doubtful' or 'bad', based on how well the modelled curve matched the data points and whether the time-course of the contrastenhancement curve was sensible (Table 4.2).

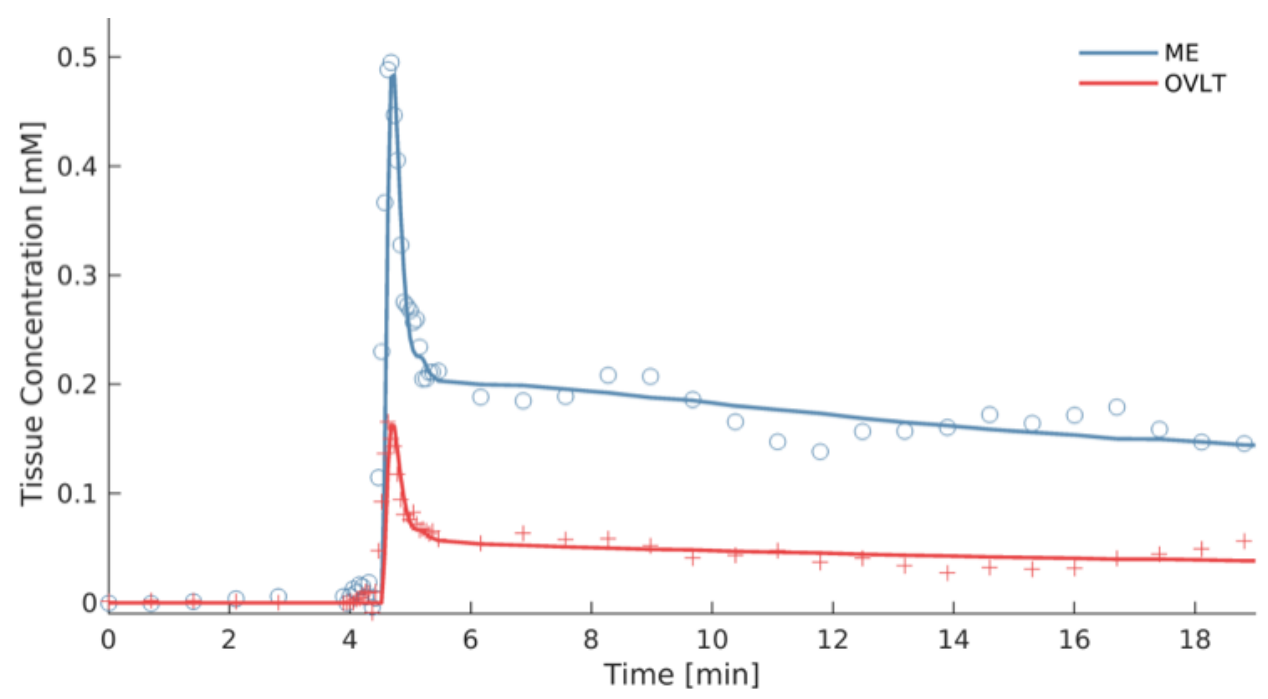

Figure 4.4. Example of time-concentration curves with good model fits obtained in a secretory (median eminence (ME)) and a sensory (organum vasculosum of the lamina terminalis (OVLT)) circumventricular organ using the extended Tofts model. Note the higher temporal sampling for 1.5 minute during the initial steep concentration changes and the slower sampling before and after this period.

The graphical Patlak method was used to calculate the leakage rate (influx) of the contrast agent into the interstitial space of the white and grey matter. This method assumes no reflux from the brain back to the blood and has been demonstrated to be most suitable for assessing pharmacokinetic parameters of normal-appearing brain tissue (37). Therefore, we applied the graphical Patlak method to assess $\mathrm{K}^{\text {trans }}\left[\mathrm{min}^{-1}\right]$ and $\mathrm{v}_{\mathrm{p}}[-]$ in each voxel in the white matter and grey matter. As not in all voxels significant transfer from blood to brain could be measured due to low $\mathrm{K}^{\text {trans }}$ values in combination with the strong influence of noise, histograms of the $\mathrm{K}^{\text {trans }}$ values in the white and grey matter were created. These histograms were subsequently corrected for noise (19), after which the mean $\mathrm{K}^{\text {trans }}$ was calculated as a representative measure for the permeability in the whole white and grey matter region. This approach has been applied previously in healthy 
Chapter 4

controls (19). For the CVOs, the graphical Patlak method did not appear to be best suitable, as due to their exchange function, reflux must also be taken into account for these structures.

In addition to pharmacokinetic analyses, one- and ten-minute areas under the curve (AUCs) $[\mu \mathrm{M} \cdot \mathrm{min}]$ were calculated as proxies of gadolinium-based contrast agent wash-in during the circulation phase, and retention during the accumulation phase, respectively. Contrary to the pharmacokinetic parameter, the AUC is not dependent on the type of pharmacokinetic analysis applied and can serve as a data-driven, thus model-free, approach for characterization of contrast enhancement.

\subsubsection{Statistics}

To ensure that the transfer constant $\left(\mathrm{K}^{\text {trans }}\right)$ of the CVOs gave a realistic representation of the data, the analyses were performed excluding the values obtained from fits that were classified as 'bad'. Post-hoc, the analyses were repeated also excluding any fits for which it could be doubted whether they were sufficiently good, to see if this would change the results.

Since our participant sample was relatively small and the transfer constants were not normally distributed for each ROI, non-parametric tests were conducted (Part 1: Wilcoxon signed-rank test; Part 2: Mann-Whitney test). All statistical analyses used a level of significance of $\mathrm{p}<0.05$, and were performed with commercial software (SPSS , version 24.0, IBM Corp., Armonk, NY, USA).

\subsubsection{Part 1:Transfer constants of the CVOs and normal-appearing brain matter}

We expected the CVOs to have strong contrast enhancement and significantly positive transfer constants, so a transfer constant significantly higher than 0 .

The transfer constants and AUCs were calculated for all CVOs separately, but also for all secretory CVOs combined and all sensory CVOs combined. The Wilcoxon signedrank test was conducted, comparing the $\mathrm{K}^{\text {trans }}$ in the secretory and sensory CVOs to a hypothesized median of 0 . If a significant result was obtained, post-hoc analyses were conducted to see which specific CVOs were significantly different from 0.

As additional analyses, Wilcoxon signed-rank tests were used to compare the $\mathrm{K}^{\text {trans }}$ between the secretory and sensory CVOs, and between the CVOs and the white and grey 
matter. Again, a significant result was followed by post-hoc analyses to determine which specific CVOs had a significantly different permeability from the other CVOs, or from the white or grey matter.

\subsubsection{Part 2: Age differences}

For the second part, we investigate whether the transfer constant ( $\mathrm{K}^{\text {trans }}$ ) differed between the older and middle-aged group.

For this between-subjects test, the Mann-Whitney test was used and the difference between the older and middle-aged group was assessed within the combined secretory CVOs, combined sensory CVOs, white matter and grey matter. If a significant result was obtained for either the combined secretory or sensory CVOs, post-hoc analyses were conducted to see for which specific CVOs the age groups differed most strongly.

\subsection{Results}

\subsection{1. $\quad$ Concentration curves of different tissue types}

We depicted the temporal enhancement profile in CVO tissue and normal-appearing brain matter, as well as muscle tissue (Figure 4.5). In the neck muscle tissue, gadoliniumbased contrast agent concentration steadily increased (dotted green line). However, the CVO concentration time curves displayed a steep peak followed by gradual decline, which are features also seen in the white and grey matter concentration curves. In this typical pattern, the contrast first quickly circulates through the brain, followed by a slow spread (dispersion). The concentration curves of the secretory CVOs ME and PG showed less steep decline after the initial circulation peaks than the concentration curve of the VIF (Figure 4.5C), which is indicative of retention of contrast medium in the CVOs. For the secretory $\mathrm{NH}$ structure, retention is also visible by a broadening (hump) after the initial peak (Figure 4.5C). 
A

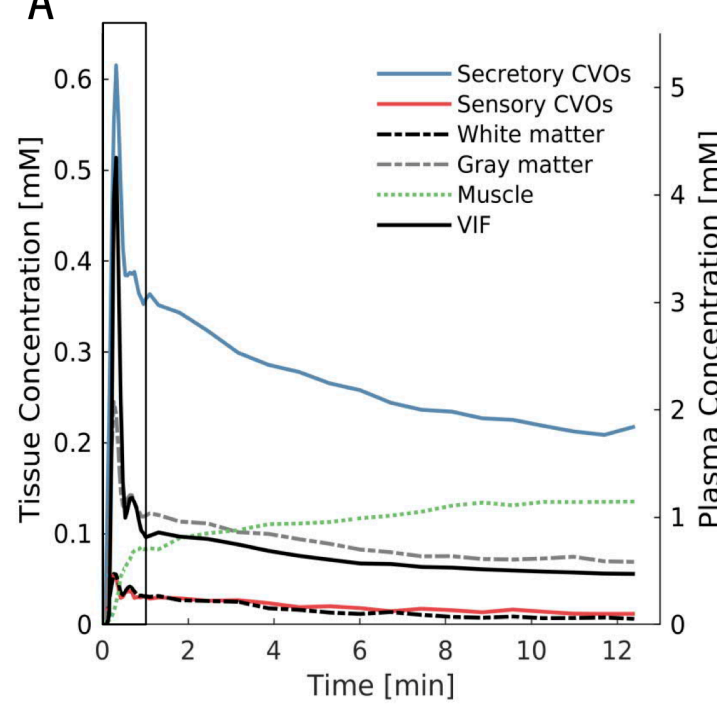

C

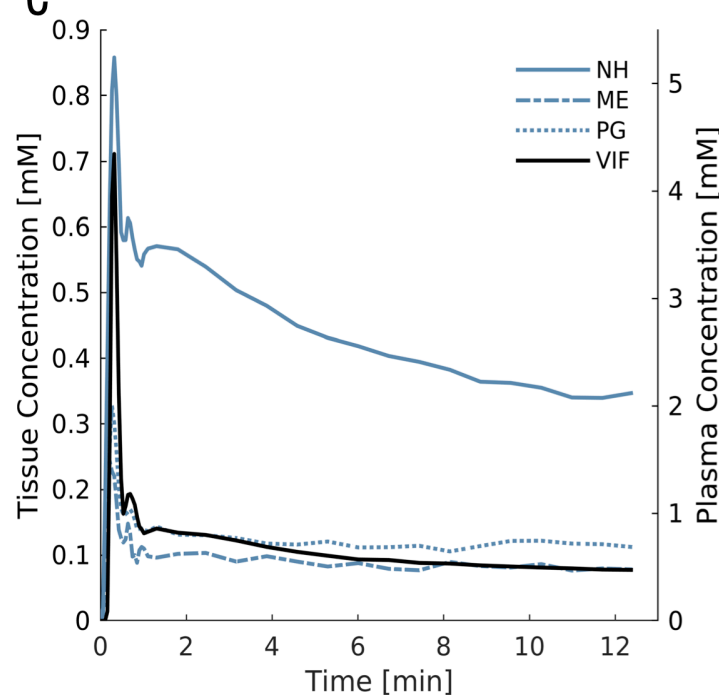

B

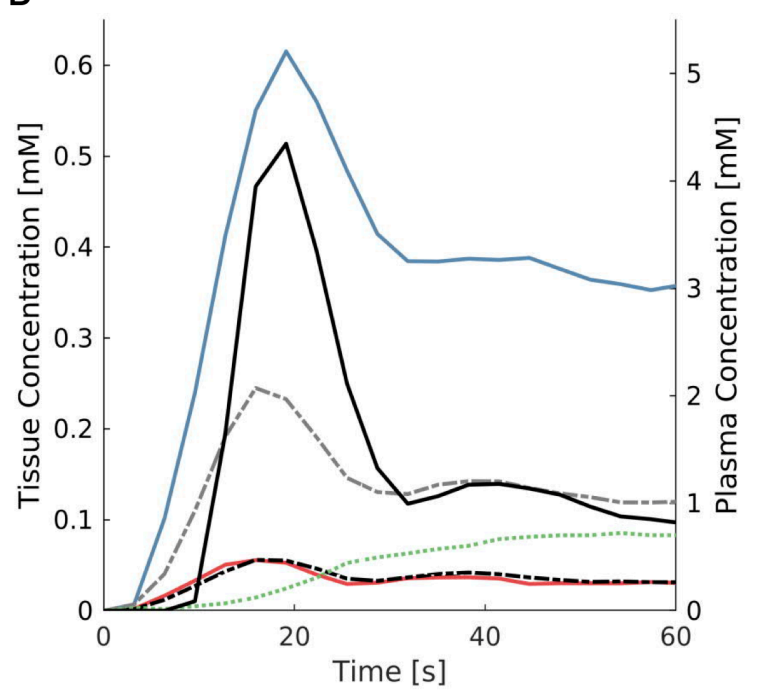

D

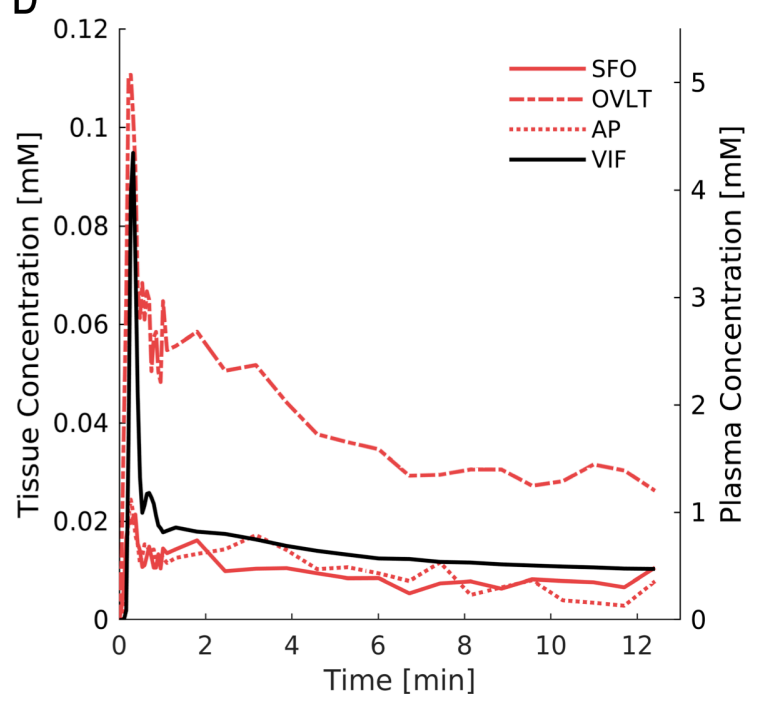

Figure 4.5. Time course of the gadolinium-based contrast agent concentration in different tissue types and the sagittal sinus (vascular input function (VIF)) averaged over all participants. $A$ and B: In the combined secretory and combined sensory circumventricular organs, white and grey matter and neck muscle since arrival to end of sequence (A) and detailed for the first minute (B); $C$ : In the secretory circumventricular organs neurohypophysis (NH), median eminence (ME) and pineal gland (PG); and D: In the sensory circumventricular organs subfornical organ (SFO), organum vasculosum of the lamina terminalis (OVLT), and area postrema (AP).

\subsubsection{Transfer constants of the CVOs}

For the secretory CVOs, significantly positive transfer constants ( $\left.\mathrm{K}^{\text {trans }}\right)$ were found $(\mathrm{p}<$ .001; Table 4.2). Post-hoc analyses revealed that this was the case for all secretory CVOs $(\mathrm{NH}, \mathrm{ME}$ and PG; all $\mathrm{p}$ values $\leq .001)$. 
Also in the sensory CVOs, significantly positive transfer constants were found $(\mathrm{p}=$ .007; Table 4.2). Post-hoc analyses revealed that this was the case for the OVLT and AP (all $p$ values $\leq .007)$, but not for the SFO ( $p=.208)$. The SFO was also the organ for which the least good fits were obtained $(n=8)$, with a lower permeability possibly making the data more susceptible to noise and therefore harder to estimate.

Repeating the analyses while also excluding the cases for which the goodness of fit could be doubted, did not change the results.

The values for the blood plasma fraction $\left(v_{p}\right)$ and the interstitial space fraction $\left(v_{e}\right)$ can be found in Supplement 2.

Table 4.2. The median and interquartile range $\left(25^{\text {th }}-75^{\text {th }}\right.$ percentile) of the transfer constant ( $\left.\mathrm{K}^{\text {trans }}\right)$ and the number of good fits measured in various regions-of-interest (ROIs)

\begin{tabular}{|c|c|c|c|}
\hline ROI & $\begin{array}{l}\text { ETM K } K^{\text {trans }} \\
{\left[\mathrm{min}^{-1}\right]}\end{array}$ & $25^{\text {th }}-75^{\text {th }}$ percentile & $\begin{array}{l}\text { Number of good fits } \\
\text { from a total of } 20\end{array}$ \\
\hline Secretory & $.22^{*}$ & $.17-.33$ & 19 \\
\hline NH & $.40^{*}$ & $.29-.63$ & 19 \\
\hline ME & $.048^{*}$ & $.032-.11$ & 19 \\
\hline PG & $.078^{*}$ & $.033-.12$ & 16 \\
\hline Sensory & $.026^{*}$ & $.021-.036$ & 10 \\
\hline SFO & $1.8 \cdot 10^{-3}$ & $-.69 \cdot 10^{-3}-25 \cdot 10^{-3}$ & 8 \\
\hline OVLT & $.019 *$ & $.0091-.034$ & 17 \\
\hline \multirow[t]{2}{*}{ AP } & $10 \cdot 10^{-3 *}$ & $.87 \cdot 10^{-3}-80 \cdot 10^{-3}$ & 10 \\
\hline & $\begin{array}{l}\text { Patlak Ktran } \\
{\left[\mathrm{min}^{-1}\right]}\end{array}$ & & \\
\hline White matter & $4.1 \cdot 10^{-7 *}$ & $.52 \cdot 10^{-7}-9.3 \cdot 10^{-7}$ & N.A. ${ }^{\dagger}$ \\
\hline Grey matter & $13 \cdot 10^{-7 *}$ & $5.1 \cdot 10^{-7}-17 \cdot 10^{-7}$ & N.A. ${ }^{\dagger}$ \\
\hline
\end{tabular}

Abbreviations: $\mathrm{NH}=$ neurohypophysis; $\mathrm{ME}=$ median eminence; $\mathrm{PG}=$ pineal gland; $\mathrm{SFO}=$ subfornical organ; OVLT = organum vasculosum of the lamina terminalis; $\mathrm{AP}=$ area postrema * $\mathrm{K}^{\text {trans }}$ significantly larger than $0(\mathrm{p}<.05)$

† Goodness of fit not applicable as a voxelwise Patlak method followed by noise correction using histogram approach was used 
Chapter 4

\subsubsection{Transfer constants in the secretory CVOs compared to the sensory CVOs}

The secretory CVOs had higher transfer constants than the sensory structures $(p<.001)$. Even comparing the secretory CVO with the lowest $\mathrm{K}^{\text {trans }}(\mathrm{ME})$ to the sensory CVO with the highest $\mathrm{K}^{\text {trans }}$ (OVLT), revealed that the ME still had a significantly higher transfer constant than the OVLT ( $\mathrm{p}=.014)$, so all secretory CVOs had significantly higher transfer constants than each sensory CVO.

\subsubsection{Transfer constants in the CVOs compared to the normal-appearing brain} matter

Comparing the CVOs to the white and grey matter gave results comparable to the main analyses, with the transfer constants being significantly larger in the CVOs than in the white matter and grey matter (all p values $\leq .004$ ), except for the SFO (both $p$ values = $.250)$.

The model-independent check with AUC values also found stronger contrast enhancement in the CVOs than in the white matter, but only for the secretory structures, which also had the highest permeability parameters when applying the pharmacokinetic analysis. The NH was the only structure that showed significantly stronger contrast enhancement than the grey matter.

Comparing the AUCs of the secretory and sensory CVOs gave the same results as comparing the transfer constants, with the secretory CVOs having significantly stronger contrast enhancement than the sensory CVOs.

These results can be found in Supplement 3.

\subsubsection{Age effect}

We found no significant differences in transfer constants between the older and middleaged group in the secretory CVOs and white matter (all p values $\geq .549$ ). The age effect did approach significance in the sensory CVOs (older: $n=6$, middle-aged: $n=4 ; p=.067$ ), with the older group tending towards a lower permeability, and grey matter (older: $\mathrm{n}=$ 10, middle-aged: $\mathrm{n}=10 ; \mathrm{p}=.051$ ), with the older group tending towards a higher permeability.

Repeating the analyses while also excluding any fits for which it could be doubted whether they were sufficiently good, gave a significant difference for the sensory CVOs 
(older: $\mathrm{n}=5$, middle-aged: $\mathrm{n}=4 ; \mathrm{p}=.032$ ), with the older group having significantly lower permeability than the middle-aged group.

\subsection{Discussion}

\subsubsection{Main findings}

In this study, we investigated the feasibility of applying dual-time resolution DCE MRI with a gadolinium-based contrast agent and pharmacokinetic modelling to assess permeability of the CVOs. It was possible to measure gadolinium-based contrast enhancement in these small structures and successfully modelled the temporal uptake curves and derived the influx rate in terms of the transfer constant. Our results demonstrated that positive transfer constants, which were significantly higher than those of the white and grey matter, could be measured in all CVOs, except for the SFO. Moreover, the transfer constants for the secretory CVOs were significantly higher than for the sensory CVOs. We could not demonstrate any clear significant pharmacokinetic differences in any of the CVOs between the age groups.

\subsubsection{Stronger contrast enhancement in the secretory CVOS}

Due to absence of the BBB in the highly fenestrated capillaries, all CVOs were expected to have significantly positive transfer constants, and our results confirmed this hypothesis. The secretory CVOs, and especially the NH as largest peptide-releasing structure, had significantly stronger contrast enhancement and higher transfer constants.

The secretory CVOs being more permeable than the sensory type is supported by previous neurobiological studies. A study in mice previously found higher vascular permeability values in the secretory CVOs relative to the sensory CVOs when using lowmolecular-weight contrast agents (fluorescein isothiocyanate (FITC), molecular weight = $0.39 \mathrm{kDa}$, and Evans Blue, molecular weight $=0.96 \mathrm{kDa}$ ), which the researchers attributed to large secretion of peptides into the blood circulation by secretory CVOs (24). Moreover, a follow-up mice study found evidence that astrocyte-tanycyte connections possibly form an alternative barrier in the sensory CVOs (38). In the human brain, post-contrast gadolinium studies were conducted to assess CVO visibility $(5,18)$. The presence of contrast enhancement in the CVOs on post-contrast T1-weighted 3 Tesla MRI was assessed, as the CVOs were speculated to be mistaken for pathology-related abnormal contrast enhancement (5). The ME, NH and PG were visible in 100\%, $96 \%$ and $84 \%$ of the 
Chapter 4

cases, respectively. The OVLT was visible in $34 \%$ of the cases, while the AP and SFO were hardly ever visualized (2\% and 1\%, respectively) (5). A similar study using post-contrast 3D T2-weighted FLAIR 3T MRI images had comparable outcomes (18). The results of the current study correspond to the results of these previous studies.

The stronger contrast enhancement in the secretory and weaker enhancement in the sensory CVOs found in our study could also have alternative explanations. Blood flow in the sensory CVOs is slower relative to the rest of the brain, for the blood plasma to have better access to the receptors (4). With slower blood flow, less contrast agent may reach the sensory CVOs.

Another confounding factor could be partial volume effects, due to the small size of the structures compared to the voxel sizes of MRI. The CVOs that seem to have the highest transfer constants, the NH and PG, are also the CVOs in close proximity to large vessels with strong contrast enhancement. Partial volume effects of these large vessels might contribute to the high transfer constants found in these CVOs. The presence of a large vessel in the ROI is reflected by obtaining a higher blood plasma volume (Supplement 2, Table 3.1). The ME, which does not seem to lie close to a large vessel, still has a higher transfer constant than the sensory CVOs. Moreover, an extra check in which the transfer constants were calculated for ROIs with a similar proximity to the large vessels, but not containing any CVO tissue, resulted in lower transfer constants compared to the values found for the secretory CVO ROIs. Therefore, while partial volume effects from the large vessels might influence the transfer constants, this demonstrates that these effects cannot fully account for the higher transfer rates found in the secretory CVOs. The sensory CVOs, being smaller than the NH and PG (Table 1 (2)), may experience stronger partial volume effects from the surrounding brain tissue. Due to these partial volume effects, measurements in the sensory CVOs could represent an underestimation. To really understand to what extent partial volume effects from large vessels or surrounding tissue influence the results, more in depth research is necessary. To address the influence of partial volume effects, optimizing the technique with a higher spatial resolution by using ultra-high-field MRI, or applying a more sophisticated pharmacokinetic model controlling for cerebral blood flow in each ROI (39), could be an interesting methodological improvement for future studies. 


\subsubsection{Age effect}

We expected that the CVOs would have less contrast enhancement in the older group, due to a decrease in blood flow. We did not find a clear significant difference in permeability when comparing the older and middle-aged group. A decrease in blood flow could possibly decrease contrast uptake, but it remains unknown whether blood flow is sufficiently reduced in the older subpopulation to display such an effect. Also, age-related vascular alterations might increase permeability (40) and compensate for the reduced blood flow effect.

The age effect did approach significance in the sensory CVOs $(\mathrm{p}=.067)$ and grey matter $(p=.051)$. In the older group, the sensory CVOs tended to be less permeable, suggesting that these structures might be more susceptible to age-related blood flow reduction decreasing permeability. In the grey matter, however, the older group showed a trend towards higher permeability, indicating that this tissue type is more susceptible to age-related vascular alterations increasing permeability, as previous studies have demonstrated $(41,42)$. However, it is important to note that the result for the sensory CVOs is based on very small group sizes (older: $n=6$, middle-aged: $n=4$ ), and therefore needs further confirmation.

The difficulty to detect a clear age effect could also be because the participants selected from the MAAS study might represent a relatively healthy subsample of the general population, i.e. long-lasting study participants representing 'survivors' in an above-average health state. As any age effect is expected to be subtle in such a subsample, it may well be that our study with 10 participants in each group had insufficient power to detect this effect. Especially in the sensory CVOs, exclusion of 'bad' fits lead to very small sample sizes, and based on the current study we cannot make any definite claims about the presence of an age effect.

\subsubsection{Additional considerations}

We used the ETM to model the CVO time-courses, as, from a theoretical perspective, this model seemed most suitable to the CVOs (blood compartment and interstitial compartment with bidirectional exchange between these compartments (35)), and gave the best fit to our data. However, the ETM can only be used in case of weak vascularization or high perfusion $(43,44)$. In tissues with either high vascularization or very slow or very 
fast exchange, the ETM can still give a good fit to the data, but the derived pharmacokinetic parameters can be unreliable (44). Therefore, without the certainty that the tissue has either low vascularization or high perfusion, the interpretation of the pharmacokinetic parameters can be misleading. Cerebral tissue is in general highly perfused (45). For the white and grey matter, for instance, the Patlak method has been established to be the preferred model (37), and this model is also intended for highly perfused tissues (43).The Patlak method is not suitable for the CVOs though, due to the added assumption of no reflux from the brain back to the blood, which is violated in the CVOs with their exchange function. Therefore, we feel that the application of the ETM to the CVO data was justified (44).

While we applied the ETM to each CVO ROI, an alternative approach would be voxelby-voxel model selection (46). According to this approach, when a higher-order model is applied (a model with three or four parameters), this model is routinely tested against lower-order models or plausible alternative models of the same order in each voxel (46, 47). The four commonly used models in DCE MRI are the Patlak model ( $K^{\text {trans }}$ and $\left.v_{p}\right)$, the classical Tofts model (TM; $\mathrm{K}^{\text {trans }}$ and $\mathrm{v}_{\mathrm{e}}$ ), the extended Tofts model (ETM; $\mathrm{K}^{\text {trans }}, \mathrm{v}_{\mathrm{p}}$ and $\mathrm{v}_{\mathrm{e}}$ ) and the two-compartmental exchange model (2CXM; $\mathrm{K}^{\text {trans }}, \mathrm{v}_{\mathrm{p}}, \mathrm{V}_{\mathrm{e}}$ and $\mathrm{F}_{\mathrm{p}}$ (blood plasma flow)) (43). As the Patlak model assumes no reflux and the TM is a one-compartment model with negligible blood volume (35), these models are not adequate as they contradict CVO functionality $(4,6)$. However, for future studies, it would be interesting to explore the use of voxel-by-voxel model selection and to compare the fits obtained with the ETM and 2CXM, and discern CVO structures from the surrounding tissue based on physiological measures.

We assessed permeability using the gadolinium-based contrast agent gadobutrol. Pathogens, such as prions and amyloid- $\beta$, can be found as soluble proteins in the blood $(48,49)$, but permeability characteristics for these pathogens are not necessarily similar to those of gadobutrol, as the molecular weight of prion proteins (27-30 kDa (50)) or amyloid- $\beta$ peptides ( $4 \mathrm{kDa}(51)$ ) is higher than the weight of gadobutrol (0.61 kDa (52)). Immunohistochemistry studies in mice have demonstrated that permeability in the CVOs is size-selective, and have found different results with low-molecular weight $(<10 \mathrm{kDa})$ and high-molecular-weight $(\geq 10 \mathrm{kDa})$ tracers $(38,53,54)$. Studies with high-molecular weight contrast agents should be conducted to confirm these findings in vivo in humans. 


\subsection{Conclusion}

In this study, dual-time resolution DCE MRI was introduced as a possible method to assess CVO permeability. With this method, significantly positive transfer constants could be measured in the CVOs, and the CVOs were shown to have stronger contrast enhancement relative to the normal-appearing brain matter. This observation indicates that current measurements confirm the often-held assumption of highly permeable CVOs, with the secretory CVOs being most permeable. The CVOs are often referred to as 'windows of the brain', and pathogens may enter through these windows and trigger an inflammatory response. More advanced ways to assess CVO permeability might eventually help blocking entrance of pathogens, determine factors that disturb homeostasis and possibly contribute to initiatives to overcome neurodegenerative disorders.

\subsection{Supplements}

Supplement 1: Participant characteristics

Supplement 2: Values for the blood plasma fraction $\left(\mathrm{v}_{\mathrm{p}}\right)$ and interstitial space fraction $\left(\mathrm{V}_{\mathrm{e}}\right)$ Supplement 3: Contrast enhancement in the CVOs and normal-appearing brain matter

\subsection{Acknowledgements}

The authors would like to acknowledge the participants for their contribution to scientific research and give special thanks to Jos M.G.M. Slenter and Jan H. Jungerius for ongoing software, hardware and network support.

\subsection{Declaration of interest}

The authors declare that they have no competing interests.

\subsection{Ethical approval and consent}

The study was approved beforehand by the medical ethics review committee of Maastricht University Medical Center (approval number: METC163050). All participants provided written informed consent beforehand regarding participation and publication of the data. 
Chapter 4

\subsection{Supplement 1: Participant characteristics}

Table 4.3. Participant characteristics of the two age groups

\begin{tabular}{lll}
\hline & Middle-aged & Old \\
\hline $\mathbf{N}$ & 10 & 10 \\
Age [years] & $53.5(50.3-55.3)$ & $73.5(71.8-78.0)$ \\
MMSEa $^{\mathbf{2}}$ & $29.5(29.0-30.0)$ & $29.0(27.8-30.0)$ \\
\%WMH Fazekas $^{\mathbf{b}}$ & & \\
$\mathbf{0 / 1 / 2 / 3}$ & $10 / 80 / 0 / 10$ & $0 / 70 / 30 / 0$ \\
\% Diabetes & 0 & 30 \\
\% Smoker & 20 & 20 \\
\hline
\end{tabular}

Notation: median $\left(25^{\text {th }}-75^{\text {th }}\right.$ percentile $) /$ percentage

a: Mini-Mental State Examination (MMSE (55)): maximum score $=30$, cognitively normal $\geq 25$

b: Fazekas scale with a visual rating score of white matter hyperintensity (WMH) load (56): $0=$ absent: none or a single punctuate WMH lesion; 1 = mild: multiple punctuate lesions; $2=$ moderate: beginning of confluency of lesions; 3 = severe: large confluent lesions 


\subsection{Supplement 3: Values for the blood plasma fraction $\left(v_{p}\right)$ and interstitial space fraction $\left(v_{e}\right)$}

Table 4.4. The median and interquartile range $\left(25^{\text {th }}-75^{\text {th }}\right.$ percentile $)$ of the blood plasma fraction $\left(v_{p}\right)$ and interstitial space fraction $\left(v_{e}\right)$ measured in various regions-of-interest (ROIs)

\begin{tabular}{|c|c|c|c|c|}
\hline ROI & $\begin{array}{l}\text { ETM vp } \\
{\left[\min ^{-1}\right]}\end{array}$ & $\begin{array}{l}25^{\text {th }}-75^{\text {th }} \\
\text { percentile }\end{array}$ & $\begin{array}{l}\text { ETM ve } \\
{\left[\mathrm{min}^{-1}\right]}\end{array}$ & $\begin{array}{l}25^{\text {th }}-75^{\text {th }} \\
\text { percentile }\end{array}$ \\
\hline Secretory & .11 & $.083-.25$ & .28 & $.20-.32$ \\
\hline $\mathrm{NH}$ & .17 & $.11-.33$ & .42 & $.30-.53$ \\
\hline ME & .058 & $.026-.094$ & .090 & $.076-.13$ \\
\hline$P G$ & .098 & $.056-.13$ & .14 & $.12-.19$ \\
\hline Sensory & .022 & $.015-.045$ & .029 & $.025-.045$ \\
\hline SFO & .018 & $.006-.056$ & .031 & $.019-1.0$ \\
\hline OVLT & .030 & $.015-.060$ & .037 & $.018-.071$ \\
\hline $\mathrm{AP}$ & .040 & $.008-.072$ & .050 & $.033-.13$ \\
\hline
\end{tabular}

Patlak vp

$\left[\min ^{-1}\right]$

White matter $\quad .010$

$.006-.012 \quad$ N.A. $*$

N.A. ${ }^{\dagger}$

Grey matter $\quad .025$

$.019-.029 \quad$ N.A. $*$

N.A. ${ }^{\dagger}$

Abbreviations: $\mathrm{NH}=$ neurohypophysis; $\mathrm{ME}=$ median eminence; $\mathrm{PG}=$ pineal gland; $\mathrm{SFO}=$ subfornical organ; OVLT = organum vasculosum of the lamina terminalis; $\mathrm{AP}=$ area postrema ₹ $\mathrm{V}_{\mathrm{e}}$ not available as this parameter is not obtained using the Patlak method

† Goodness of fit not applicable as a voxelwise Patlak method followed by noise correction using histrogram approach was used 
Chapter 4

\subsection{Supplement 4: Contrast enhancement in the CVOs and normal appearing brain matter}

Table 4.5. Median and interquartile range $\left(25^{\text {th }}-75^{\text {th }}\right.$ percentile $)$ of 1 -minute and 10 minute area under the curve (AUC1 and AUC10) measured in various regions-of-interest (ROIs)

\begin{tabular}{cllll}
\hline ROI & $\begin{array}{l}\text { AUC1 } \\
{[\boldsymbol{\mu M} \cdot \mathbf{m i n}]}\end{array}$ & $\mathbf{2 5}^{\text {th }}-\mathbf{7 5}^{\text {th }}$ percentile & $\begin{array}{l}\text { AUC10 } \\
{[\boldsymbol{\mu M} \cdot \mathbf{m i n}]}\end{array}$ & $\mathbf{2 5}^{\text {th }} \mathbf{\mathbf { 7 5 } ^ { \text { th } }}$ percentile \\
\hline Secretory & $418^{\dagger \ddagger}$ & $260-534$ & $2947^{\ddagger \ddagger}$ & $1991-3482$ \\
NH & $638^{\dagger \ddagger}$ & $438-802$ & $4589^{\ddagger \ddagger}$ & $2898-5394$ \\
ME & $148^{\dagger}$ & $82-230$ & $940^{\dagger}$ & $597-1304$ \\
PG & $192^{\dagger}$ & $111-270$ & $1224^{\dagger}$ & $763-1778$ \\
Sensory & $38^{\ddagger}$ & $4-55$ & $223^{\ddagger}$ & $46-302$ \\
SFO & $17^{\ddagger}$ & $2-50$ & $119^{\ddagger}$ & $34-160$ \\
OVLT & $69^{\ddagger}$ & $31-113$ & $410^{\ddagger}$ & $207-654$ \\
AP & $17^{\ddagger}$ & $0-63$ & $112^{\ddagger}$ & $9-370$ \\
White matter & 41 & $23-48$ & 176 & $91-234$ \\
Grey matter & 155 & $77-198$ & 937 & $469-1218$ \\
\hline
\end{tabular}

$\uparrow$ significantly $(\mathrm{p}<.05)$ different from white matter, $\ddagger$ significantly $(\mathrm{p}<.05)$ different from grey matter. Abbreviations: $\mathrm{NH}=$ neurohypophysis; $\mathrm{ME}=$ median eminence; $\mathrm{PG}=$ pineal gland; $\mathrm{SFO}=$ subfornical organ; OVLT = organum vasculosum of the lamina terminalis; $\mathrm{AP}=$ area postrema; AUC $=$ area under the curve.

For the secretory CVOs, the 1-minute and 10-minute AUCs were significantly larger than for the white and grey matter (Table 4.5, all p-values < .001). Post-hoc analyses demonstrated that all secretory CVOs had significantly higher values than the white matter (all p-values <.001), while the difference with the grey matter was only due to the $\mathrm{NH}$ (all p-values <.001).

For the sensory CVOs, the 1-minute and 10-minute AUCs were not significantly different compared to the white matter, and were actually smaller than for the grey matter (Table 4.1., all p-values <.001). 


\subsubsection{Age effect}

We found no significant differences between the older and middle-aged group in any of the brain regions (secretory CVOs; sensory CVOs, white matter; grey matter) for the 1minute or 10-minute AUC (all p-values $\geq .165$ ).

\subsection{References}

1. Benarroch EE. Circumventricular organs Receptive and homeostatic functions and clinical implications. Neurology. 2011;77(12):1198-204.

2. Duvernoy HM, Risold P-Y. The circumventricular organs: an atlas of comparative anatomy and vascularization. Brain research reviews. 2007;56(1):119-47.

3. Oldfield BJ, McKinley MJ. Circumventricular organs. The rat nervous system: Elsevier; 2015. p. 315-33.

4. Sisó S, Jeffrey M, González L. Sensory circumventricular organs in health and disease. Acta neuropathologica. 2010;120(6):689-705.

5. Horsburgh A, Massoud TF. The circumventricular organs of the brain: conspicuity on clinical 3T MRI and a review of functional anatomy. Surgical and Radiologic Anatomy. 2013;35(4):343-9.

6. Gross PM, Weindl A, Knigge KM. Peering through the windows of the brain. Journal of Cerebral Blood Flow \& Metabolism. 1987;7(6):663-72.

7. Langlet F, Mullier A, Bouret SG, Prevot V, Dehouck B. Tanycyte-like cells form a bloodcerebrospinal fluid barrier in the circumventricular organs of the mouse brain. Journal of Comparative Neurology. 2013;521(15):3389-405.

8. Cameron OG. Visceral brain-body information transfer. NeuroImage. 2009;47(3):787-94.

9. Cottrell GT, Ferguson AV. Sensory circumventricular organs: central roles in integrated autonomic regulation. Regulatory peptides. 2004;117(1):11-23.

10. McKinley MJ. The sensory circumventricular organs of the mammalian brain: subfornical organ, OVLT and area postrema: Springer Science \& Business Media; 2003.

11. Li N-C, Lee A, Whitmer RA, Kivipelto M, Lawler E, Kazis LE, et al. Use of angiotensin receptor blockers and risk of dementia in a predominantly male population: prospective cohort analysis. Bmj. 2010;340:b5465.

12. Kristensson K, Nygård M, Bertini G, Bentivoglio M. African trypanosome infections of the nervous system: parasite entry and effects on sleep and synaptic functions. Progress in neurobiology. 2010;91(2):152-71.

13. Steinman L. Elaborate interactions between the immune and nervous systems. Nature immunology. 2004;5(6):575.

14. Buller KM. Circumventricular Organs: Gateways to the Brain Role Of Circumventricular Organs In Pro-Inflammatory Cytokine-Induced Activation Of The Hypothalamic-Pituitary-Adrenal Axis. Clinical and Experimental Pharmacology and Physiology. 2001;28(7):581-9. 


\section{Chapter 4}

15. Swaab DF, Bao A-M, Lucassen PJ. The stress system in the human brain in depression and neurodegeneration. Ageing research reviews. 2005;4(2):141-94.

16. Sisó S, González L, Jeffrey M. Neuroinvasion in prion diseases: the roles of ascending neural infection and blood dissemination. Interdisciplinary perspectives on infectious diseases. 2010;2010.

17. Sisó S, Jeffrey M, González L. Neuroinvasion in sheep transmissible spongiform encephalopathies: the role of the haematogenous route. Neuropathology and applied neurobiology. 2009;35(3):232-46.

18. Azuma M, Hirai T, Kadota Y, Khant ZA, Hattori Y, Kitajima M, et al. Circumventricular organs of human brain visualized on post-contrast 3D fluid-attenuated inversion recovery imaging. Neuroradiology. 2018;60(6):583-90.

19. van de Haar HJ, Burgmans S, Jansen JF, van Osch MJ, van Buchem MA, Muller M, et al. Blood-brain barrier leakage in patients with early Alzheimer disease. Radiology. 2016;281(2):527-35.

20. Renkin EM. Effects of blood flow on diffusion kinetics in isolated, perfused hindlegs of cats: a double circulation hypothesis. American Journal of Physiology-Legacy Content. 1955;183(1):125-36.

21. Crone $\mathrm{C}$. The permeability of capillaries in various organs as determined by use of the indicator diffusion'method. Acta physiologica scandinavica. 1963;58(4):292-305.

22. Leenders K, Perani D, Lammertsma A, Heather J, Buckingham P, Jones T, et al. Cerebral blood flow, blood volume and oxygen utilization: normal values and effect of age. Brain. 1990;113(1):27-47.

23. Simmons GE, Suchnicki J, Rak K, Damiano T. MR imaging of the pituitary stalk: size, shape, and enhancement pattern. AJR American journal of roentgenology. 1992;159(2):375-7.

24. Morita S, Miyata S. Different vascular permeability between the sensory and secretory circumventricular organs of adult mouse brain. Cell and tissue research. 2012;349(2):589-603.

25. Jolles J, Houx P, Van Boxtel M, Ponds R. Maastricht aging study: Determinants of cognitive aging: Neuropsych Publishers Maastricht; 1995.

26. Fujisawa S, Tanaka J, Nomura M. Estrogen attenuates the drinking response induced by activation of angiotensinergic pathways from the lateral hypothalamic area to the subfornical organ in female rats. Behavioural brain research. 2001;122(1):33-41.

27. Limonta P, Maggi R, Martini L, Piva F. Role of the subcommissural organ in the control of gonadotrophin secretion in the female rat. Journal of Endocrinology. 1982;95(2):207-13.

28. Wenger T, Leonardelli J. Circadian and cyclic LHRH variations in the organum vasculosum of the lamina terminalis of female and male rats. Neuroendocrinology. 1980;31(5):331-7.

29. van de Haar HJ, Burgmans S, Jansen JF, van Osch MJ, van Buchem MA, Muller M, et al. Blood-Brain Barrier Leakage in Patients with Early Alzheimer Disease. Radiology. 2017;282(2):615.

30. Larsson HB, Courivaud F, Rostrup E, Hansen AE. Measurement of brain perfusion, blood volume, and blood-brain barrier permeability, using dynamic contrast-enhanced T(1)-weighted MRI at 3 tesla. Magn Reson Med. 2009;62(5):1270-81.

31. Fischl B. FreeSurfer. NeuroImage. 2012;62(2):774-81.

32. Jelescu IO, Leppert IR, Narayanan S, Araujo D, Arnold DL, Pike GB. Dual-temporal resolution dynamic contrast-enhanced MRI protocol for blood-brain barrier permeability measurement in enhancing multiple sclerosis lesions. J Magn Reson Imaging. 2011;33(6):1291-300. 
33. van de Haar HJ, Jansen JFA, Jeukens C, Burgmans S, van Buchem MA, Muller M, et al. Subtle bloodbrain barrier leakage rate and spatial extent: Considerations for dynamic contrast-enhanced MRI. Med Phys. 2017;44(8):4112-25.

34. van de Haar HJ, Jansen JFA, van Osch MJP, van Buchem MA, Muller M, Wong SM, et al. Neurovascular unit impairment in early Alzheimer's disease measured with magnetic resonance imaging. Neurobiol Aging. 2016;45:190-6.

35. Heye AK, Thrippleton MJ, Armitage PA, Valdés Hernández MdC, Makin SD, Glatz A, et al. Tracer kinetic modelling for DCE-MRI quantification of subtle blood-brain barrier permeability. NeuroImage. 2016;125:446-55.

36. Barnes SR, Ng TS, Santa-Maria N, Montagne A, Zlokovic BV, Jacobs RE. ROCKETSHIP: a flexible and modular software tool for the planning, processing and analysis of dynamic MRI studies. BMC medical imaging. 2015;15(1):19.

37. Cramer SP, Larsson HB. Accurate determination of blood-brain barrier permeability using dynamic contrast-enhanced T1-weighted MRI: a simulation and in vivo study on healthy subjects and multiple sclerosis patients. Journal of Cerebral Blood Flow \& Metabolism. 2014;34(10):1655-65.

38. Morita S, Furube E, Mannari T, Okuda H, Tatsumi K, Wanaka A, et al. Heterogeneous vascular permeability and alternative diffusion barrier in sensory circumventricular organs of adult mouse brain. Cell and tissue research. 2016;363(2):497-511.

39. Sourbron S, Ingrisch M, Siefert A, Reiser M, Herrmann K. Quantification of cerebral blood flow, cerebral blood volume, and blood-brain-barrier leakage with DCE-MRI. Magnetic Resonance in Medicine: An Official Journal of the International Society for Magnetic Resonance in Medicine. 2009;62(1):205-17.

40. Heiss C, Keymel S, Niesler U, Ziemann J, Kelm M, Kalka C. Impaired progenitor cell activity in agerelated endothelial dysfunction. Journal of the American College of Cardiology. 2005;45(9):1441-8.

41. Verheggen IC, de Jong JJ, van Boxtel MP, Gronenschild EH, Palm WM, Postma AA, et al. Increase in blood-brain barrier leakage in healthy, older adults. GeroScience. 2020:1-11.

42. Starr JM, Farrall AJ, Armitage P, McGurn B, Wardlaw J. Blood-brain barrier permeability in Alzheimer's disease: a case-control MRI study. Psychiatry Research: Neuroimaging. 2009;171(3):232-41.

43. Sourbron SP, Buckley DL. Classic models for dynamic contrast-enhanced MRI. NMR in Biomedicine. 2013;26(8):1004-27.

44. Sourbron SP, Buckley DL. On the scope and interpretation of the Tofts models for DCE-MRI. Magnetic resonance in medicine. 2011;66(3):735-45.

45. Detre JA, Zhang W, Roberts DA, Silva AC, Williams DS, Grandis DJ, et al. Tissue specific perfusion imaging using arterial spin labeling. NMR in Biomedicine. 1994;7(1-2):75-82.

46. Ewing JR, Bagher-Ebadian H. Model selection in measures of vascular parameters using dynamic contrast-enhanced MRI: experimental and clinical applications. NMR in Biomedicine. 2013;26(8):1028-41. 47. Donaldson SB, West CM, Davidson SE, Carrington BM, Hutchison G, Jones AP, et al. A comparison of tracer kinetic models for T1-weighted dynamic contrast-enhanced MRI: Application in carcinoma of the cervix. Magnetic Resonance in Medicine. 2010;63(3):691-700.

48. Edgeworth JA, Farmer M, Sicilia A, Tavares P, Beck J, Campbell T, et al. Detection of prion infection in variant Creutzfeldt-Jakob disease: a blood-based assay. The Lancet. 2011;377(9764):487-93. 


\section{Chapter 4}

49. Lue L-F, Guerra A, Walker DG. Amyloid Beta and Tau as Alzheimer's Disease Blood Biomarkers: Promise From New Technologies. Neurol Ther. 2017;6(Suppl 1):25-36.

50. Collinge J. Molecular neurology of prion disease. Journal of Neurology, Neurosurgery \& Psychiatry. 2005;76(7):906-19.

51. Chen G-f, Xu T-h, Yan Y, Zhou Y-r, Jiang Y, Melcher K, et al. Amyloid beta: structure, biology and structure-based therapeutic development. Acta Pharmacologica Sinica. 2017;38(9):1205.

52. Gadavist, CID=70678987 [Internet]. U.S. National Library of Medicine. 2019 [cited Nov. 13, 2019]. Available from: https://pubchem.ncbi.nlm.nih.gov/compound/Gadavist.

53. Miyata S. New aspects in fenestrated capillary and tissue dynamics in the sensory circumventricular organs of adult brains. Frontiers in Neuroscience. 2015;9(390).

54. Willis C, Garwood C, Ray D. A size selective vascular barrier in the rat area postrema formed by perivascular macrophages and the extracellular matrix. Neuroscience. 2007;150(2):498-509.

55. Folstein MF, Folstein SE, McHugh PR. "Mini-mental state": a practical method for grading the cognitive state of patients for the clinician. Journal of psychiatric research. 1975;12(3):189-98.

56. Fazekas F, Kleinert R, Offenbacher H, Schmidt R, Kleinert G, Payer F, et al. Pathologic correlates of incidental MRI white matter signal hyperintensities. Neurology. 1993;43(9):1683-. 


Chapter 5

\subsection{Abstract}

Neurovascular pathology concurs with protein accumulation, as the brain vasculature is important for waste clearance. Interstitial solutes, such as amyloid- $\beta$, were previously thought to be primarily cleared from the brain by blood-brain barrier transport. Recently, the glymphatic system was discovered, in which cerebrospinal fluid is exchanged with interstitial fluid, facilitated by the aquaporin- 4 water channels on the astroglial endfeet. Glymphatic flow can clear solutes from the interstitial space. Blood-brain barrier transport and glymphatic clearance likely serve complementary roles with partially overlapping mechanisms providing a well-conditioned neuronal environment. Disruption of these mechanisms can lead to protein accumulation and may initiate neurodegenerative disorders, for instance amyloid- $\beta$ accumulation and Alzheimer's disease. Although both mechanisms seem to have a similar purpose, their interaction has not been clearly discussed previously. This review focusses on this interaction in healthy and pathological conditions. Future health initiatives improving waste clearance might delay or even prevent onset of neurodegenerative disorders. Defining glymphatic flow kinetics using imaging may become an alternative way to identify those at risk of Alzheimer's disease.

\section{Keywords}

Blood-brain barrier, BBB, glymphatic system, clearance, amyloid- $\beta$, Alzheimer's disease, imaging

\subsection{Introduction}

Brain pathology and neurodegeneration can result from neurotoxins that enter the brain and from unremoved waste products. The blood-brain barrier (BBB) keeps out components that may disturb synaptic signalling or generate neurotoxins (1). In this way, the BBB protects the central nervous system against ion fluctuations that occur in the bloodstream and maintains the homeostasis. Interstitial solutes are transported across the local BBB by transport proteins and are then removed via the blood stream. However, with a large distance between interstitial solutes and the BBB, the water flow regulated by the glial cells must be utilized $(2,3)$. Cerebrospinal fluid (CSF) is exchanged with interstitial fluid (ISF), facilitated by the aquaporin-4 (AQP4) water channels on the astroglial endfeet. ISF is drained into the paravenous space, from which it eventually 
reaches the lymphatic nodes. This glymphatic flow can clear solutes from the interstitial space and therefore seems to have the same function as lymph outside the brain. This system was given the name glymphatic system, a merging of the words 'glial' and 'lymphatic' (4).

BBB breakdown is thought to be the underlying mechanism of small vessel disease, which refers to pathology in the small cerebral blood vessels and capillaries (5$7)$. BBB disruption also increases the amount of accumulated amyloid- $\beta$ protein $(A \beta)$ and $A \beta$ accumulation aggravates $B B B$ disruption (8). $A \beta$ is cleared from the brain through BBB transport, but recent studies have demonstrated that its clearance is also largely dependent on the glymphatic system $(3,4)$. The connections between BBB disruption, glymphatic dysfunction and $A \beta$ accumulation could provide a link between vascular pathology and early Alzheimer pathology.

\subsubsection{Blood-brain barrier}

The BBB in the cerebrovascular wall separates the blood circulation from the brain parenchyma. One component of the BBB are the endothelial cells connected by tight junctions that form a strong physical barrier (9). The endothelial cells have a luminal membrane at the side of the blood and an abluminal membrane at the side of the parenchyma (10). On the abluminal side, the endothelial cells are supported by astrocytes and pericytes (11). The endothelial cells, astrocytes and pericytes can express enzymes that can inactivate foreign substances that manage to pass through the physical barrier (12). Only small lipophilic molecules can pass the BBB by means of free diffusion. Furthermore, the BBB contains active transporter proteins and only molecules that match one of these transporters can be actively transferred across the endothelial cells.

The BBB regulates ion transport to keep the ISF optimal for neuronal functioning (13). The BBB protects the brain from ion fluctuations that occur in the bloodstream after a meal or exercise, which would otherwise disturb synaptic and axonal signalling (14). Moreover, blood components crossing the BBB can activate signalling pathways or trigger inflammatory responses that generate neurotoxins and compromise neuronal functioning $(1,15)$.

The astrocytes also have an important role in maintaining homeostasis. In normal brain activity, neurons obtain energy through glucose metabolism, which generates water (12). Water accumulation leads to tissue swelling and excess water must be 
Chapter 5

redistributed. Water passage between the endothelial cells is prevented by the tight junctions $(16,17)$. Transendothelial water transport is limited due to low expression of water channels on the endothelial membrane. The endfeet of the astrocytes, however, contain a high density of AQP4 water channels. Water flow through the AQP4 channels facilitates the redistribution of excess water via the perivascular space.

\subsubsection{Blood-brain barrier disruption}

Brain pathology often leads to loosening of the tight junctions, which compromises the BBB integrity (1). It has long been controversial whether BBB disruption is associated with older age, but recently a large-scale meta-analysis of 31 BBB permeability studies has indeed demonstrated that BBB disruption does occur during normal aging (6). Montagne et al. (2015) found a positive association between BBB leakage in the hippocampus and normal aging (18). They also demonstrated that BBB leakage in the hippocampus was associated with mild cognitive impairment and concluded that BBB disruption may contribute to dementia onset. A review of BBB permeability studies in dementia concluded that there is increased BBB leakage in individuals with dementia, especially in the presence of white matter hyperintensities and vascular pathology (19). Early Alzheimer pathology, such as $A \beta$ accumulation, can further disrupt the BBB (20).

$\mathrm{A} \beta$ is a peptide produced during normal cell metabolism and present in the blood plasma, brain ISF and CSF $(21,22)$. Influx and efflux transport mechanisms regulate the $A \beta$ concentration in the brain. Soluble, unbound $A \beta$ can move across the $B B B$ via receptor-mediated transport (23). Moreover, $A \beta$ carrier proteins can bind and sequester $A \beta$ in the plasma, ISF or $\operatorname{CSF}(23,24)$. Carrier proteins forming complexes with $A \beta$ facilitate receptor-mediated transport across the $\operatorname{BBB}(25,26)$. The blood plasma and extracellular fluid contain $A \beta$-degrading proteases (27). The $A \beta$ peptide occurs in different forms ranging from 39 to 43 amino acid. Especially unbound $A \beta_{42}$ has the tendency to form non-soluble fibrils(24). When the $A \beta$ concentration is high, fibrillary $A \beta$ aggregates, which can lead to accumulation of neurotoxic $A \beta$ plaques. Often in cases of elevated $A \beta$ concentration, changes in $A \beta$ efflux and influx transporters are observed while the amount of $A \beta$ production remains the same $(1,27)$. RAGE is an $A \beta$ influx transporter normally expressed at relatively low levels. LRP1 is the transporter normally involved in the clearance of the $A \beta$ protein across the BBB. The expression of RAGE and LRP1 changes during aging, so that $A \beta$ influx into the brain through RAGE increases, while 
$A \beta$ efflux from the brain through LRP1 decreases (28). Also members of the ATP-binding cassette $(\mathrm{ABC})$ transporter family are important contributors to the BBB and are involved in the clearance of $A \beta$ across the BBB (29). For instance, the P-glycoprotein (P-gp) encoded by the ABCB1 gene is highly expressed at the $\mathrm{BBB}$ and is involved in $\mathrm{A} \beta$ clearance $(30,31)$ (Figure 4.1b). P-gp-null mice had significantly higher levels of $A \beta$ in the brain and more $A \beta$ accumulation (30). In humans, studies have demonstrated that $A D$ patients have lower P-gp function relative to healthy controls (31). Moreover, older subjects demonstrated decreased P-gp function and expression relative to younger subjects (32, 33). Mouse models in which mice were genetically modified to lack specific $A B C$ transporters have demonstrated that more types of $A B C$ transporters are important for $A \beta$ clearance, such as ABCA1 (34) and ABCC1 (29).

Dementia research has long focused on the amyloid cascade hypothesis. This hypothesis states that $A \beta$ accumulation in the brain is the direct cause of $A D$ development (35). However, even though the current evidence demonstrates the involvement of $A \beta$ in $A D$, the available data is not conclusive that it is critical for the development of the disease. As data cannot confirm the amyloid cascade hypothesis, it has repeatedly been suggested that the hypothesis should be restated or even rejected (36-38) It may be that a third variable exists that could be the cause of $\mathrm{AD}$ development and explain the link between $\mathrm{AD}$ and $\mathrm{A} \beta$ accumulation. $\mathrm{A}$ possible candidate is a defective clearance system. Unremoved waste products can compromise BBB integrity and neuronal functioning, which could trigger brain pathology (1). $A \beta$ is among the waste products that accumulate in the brain due to defective clearance, so that $A \beta$ accumulation coincides with $A D$ development.

The brain vasculature plays an important role in clearance, as interstitial solutes are transported across the BBB and removed via the blood stream. Defective clearance as initiating factor of $\mathrm{AD}$ blurs the etiological distinction between $\mathrm{AD}$ and vascular dementia. Indeed, more researchers now claim that this distinction is not clear-cut $(39,40)$. AD is usually characterized by Alzheimer pathology, such as $A \beta$ accumulation and tau tangle formation (41). The most common vascular cause of dementia is small vessel disease, which refers to pathology in the small cerebral blood vessels and capillaries $(40,42)$. Markers of small vessel disease detected by neuroimaging are for instance white matter lesions, lacunar infarction and microbleeds (42). In reality, disorders lie on a continuum 
Chapter 5

between $\mathrm{AD}$ and vascular dementia and the expressed pathology is often a combination between Alzheimer pathology and markers of small vessel disease $(39,43)$.

BBB disruption is thought to be one of the underlying mechanism of small vessel disease $(5,6)$. Studies have now also demonstrated that BBB disruption interacts with A $\beta$ accumulation and these processes increase neurodegeneration (8). If BBB disruption has a major role in vascular causes of dementia and strengthens Alzheimer pathology, it may be a common trigger for age-related brain pathology (44). Previously, it was thought that about 75 percent of $\mathrm{A} \beta$ was cleared by $\mathrm{BBB}$ transport, and only 10 percent by the glymphatic system (45). However, two recent photon imaging studies in mice, using microscopy with fluorescent tracers, have suggested that the glymphatic system contributes to a larger part of $A \beta$ clearance than previously thought $(3,4)$. Glymphatic dysfunction may provide the link between vascular pathology and $A \beta$ accumulation and lead to new insights on the development of brain pathology.

\subsubsection{Glymphatic system}

The brain is in a state of constant high metabolic activity and needs an efficient system to remove its metabolites, also those at a larger distance from the BBB (46). This clearance system was named the glymphatic system, due to the involvement of glial cells and its functional resemblance to the lymphatic system (4).

The perivascular space is the fluid-filled cavity surrounding a penetrating vessel (40). The perivascular space surrounding a penetrating artery (referred to as the paraarterial space by Iliff et al. (2012) (4)) forms the entrance point of the glymphatic system (9). CSF runs through the perivascular space on the abluminal side of the endothelial membrane. Perivascular AQP4 channels on the astrocytic endfeet facilitate convective flow of CSF out of the perivascular space into the interstitial space $(4,9,47)$. Convective flow refers to bulk flow, or movement of a fluid where all components move together at the same speed, so the fluid itself and all that it contains (48). CSF containing waste products is exchanged with ISF. Convective flow leads ISF with waste products away from the arteries, through the parenchyma and toward the veins into the paravenous space (4, 9) (Figure $5.1(9))$. 
a

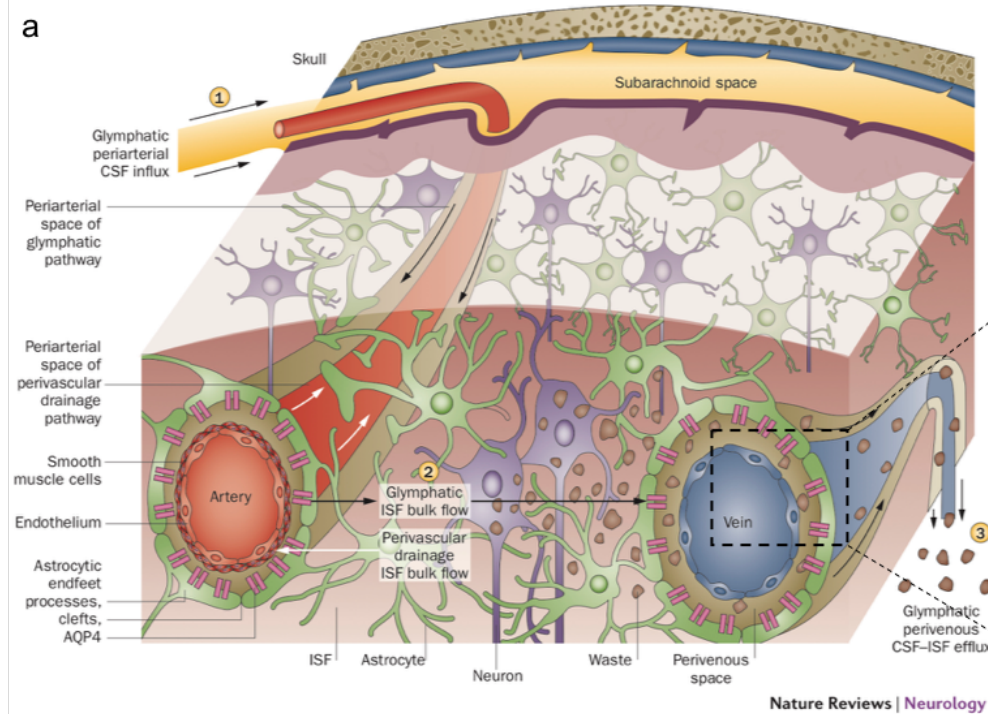

b

Perivascular space

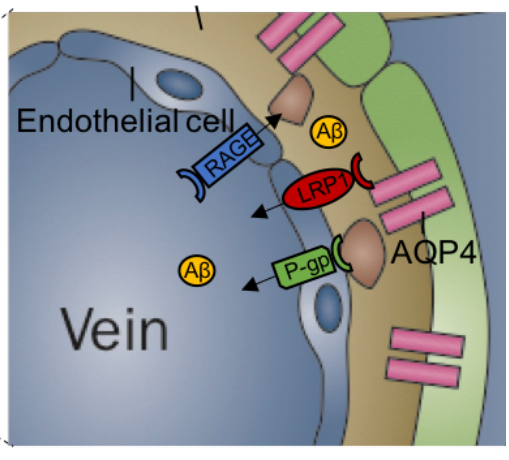

Figure 5.1. Overview of a) glymphatic clearance (9) and b) BBB transport

Adapted with permission from Springer Customer Service Center GmbH: Springer Nature, Nature Reviews Neurology, (Clearance systems in the brain - implications for Alzheimer Disease, TarasoffConway JM, Carare RO, Osorio RS, Glodzik L, Butler T, Fieremans E, et al., 2015).

Via the paravenous space, ISF with waste products eventually reaches the lymphatic nodes in the neck and enters the lymphatic circulation. Recently, the presence of functional lymphatic vessels in the brain, lining the dural sinuses, has been demonstrated (49). The researchers propose that the glymphatic system could be the first step of the brain drainage system, in which ISF is drained into the CSF, and as a second step ISF could then enter the newly identified lymphatic vessels in the dura. In mice, a tracer study has already demonstrated that these lymphatic vessels are the major outflow pathway of drainage from the brain (50). Another possibility is that CSF containing waste products circulates in the arachnoid space and flows through the arachnoid villi to the dural venous sinuses, thereby entering the blood circulation $(9,47)$. Either through the lymphatic or blood circulation, eventually solutes reach the liver where they are degraded.

\subsubsection{Glymphatic dysfunction}

Aging is associated with glymphatic dysfunction (3). Glymphatic dysfunction could be due to disregulation of water transport in the astrocytes (51). The AQP4 channels are responsible for water transport. Mice with AQP4 deletion had impaired CSF influx and lacked a pathway for CSF-to-ISF turnover (4). A $\beta$ clearance was for instance 55 percent 
less in these mice. Another study used mice with $A \beta$-overexpressing mutations (52). AQP4 deletion in these mutant mice lead to significantly higher $A \beta$ levels, while the levels of proteins involved in $A \beta$ formation or degradation remained the same, suggesting it was the clearance that was impaired. The polarization of AQP4 channels seems to be more important than the number of AQP4 channels (3). AQP4 polarization is a measure of relative AQP4 localization, with high polarization meaning high reactivity of the perivascular AQP4 channels relative to the parenchymal AQP4 channels. Depolarization is associated with glymphatic dysfunction in aged mice. Also in the post-mortem human brain, AQP4 polarization was found to be a predictor of $\mathrm{AD}, \mathrm{A} \beta$ level and Braak staging (53).

Glymphatic dysfunction could also be due to changes in CSF influx. CSF influx is dependent on arterial pulsatility $(51,54)$. In a mice study, dobutamine was used to increase the arterial pulsatility, which lead to increased CSF influx and CSF-to-ISF turnover (54). In another study, decreased vessel wall compliance, arterial pulsatility and CSF influx were demonstrated in aged mice (3). However, recent studies have demonstrated that not only inhibition of CSF influx can disrupt glymphatic clearance, but overstimulation of this influx could also be associated with glymphatic dysfunction (55, 56). Enlargement of the perivascular spaces is associated with glymphatic dysfunction (4). However, this enlargement decreases resistance to the perivascular flow and paradoxically increases CSF influx (56). Solute trapping in the perivascular space could be a possible explanation for this paradox (57). Increased CSF influx drives more solutes into the perivascular space. However, due to enlargement of perivascular spaces, AQP4 depolarization or changes in the composition of the basal lamina, these solutes cannot leave the perivascular space, combining increased CSF influx with glymphatic dysfunction.

An important consequence of glymphatic dysfunction is that $A \beta$ removal is attenuated. Synaptic activity regulates the amount of $A \beta$ in the interstitial space (58). $A \beta$ is removed again via BBB transport or glymphatic clearance. However, as glymphatic clearance decreases with age, this removal is no longer sufficient, leading to $A \beta$ deposition. $A \beta$ is deposited in the parenchyma, but also in the blood vessel walls as cerebral amyloid angiopathy (CAA) (59). CAA leads to narrowing of the perivascular space and blocks the glymphatic clearance route, which accelerates $A \beta$ accumulation (47, 
59). This vicious circle of glymphatic dysfunction, protein accumulation and perivascular narrowing may be characteristic for AD (51).

Glymphatic dysfunction could help us understand neurodegenerative disorders in which accumulation of toxic waste products contributes to disorder development (60). Glymphatic dysfunction in rats has already been associated with impaired cognitive functioning on behavioural tests $(55,61)$. MRI techniques to obtain signal from the perivascular space are being developed and could eventually image glymphatic influx and efflux and the clearance kinetics, which may make it possible to identify those at risk of neurodegenerative disorders such as AD (62).

BBB transport and glymphatic clearance seem to have a similar purpose in the removal of interstitial solutes, but still the interaction between these mechanisms has not been clearly discussed previously. Investigating their interplay and their role in brain pathology can add to our knowledge on neurodegenerative disorders and may generate new treatment options. For this narrative review, literature databases were searched for information on waste clearance from the brain, cooperation between the BBB and glymphatic system and the interaction between BBB disruption and glymphatic dysfunction.

\subsection{Methods}

To investigate how the BBB and glymphatic system interact, a literature search was conducted (63). The search terms blood-brain barrier (using MeSH terminology in PubMed and the synonyms blood-brain barriers, blood brain barrier(s), brain-blood barrier(s), brain blood barrier(s), hemato-encephalic barrier(s), hemato encephalic barrier(s) or BBB) and glymphatic were used. The search produced in total 27 results in Embase and 22 results in PubMed. After removing the duplicates, 32 papers remained. Cross-referencing was used.

All 32 papers were screened by reading the abstract and scanning through the main text. Papers were included when they met all of the following inclusion criteria: 1) discussing BBB function, 2) discussing the glymphatic system and 3) specifically focusing on their role in waste clearance. Articles were excluded when they were not available in English, German or Dutch. After screening, 18 papers remained (Figure 5.2). These papers were fully read. All information on waste clearance from the brain, cooperation 
Chapter 5

between the BBB and glymphatic system and the interaction between BBB disruption and glymphatic dysfunction was summarized.

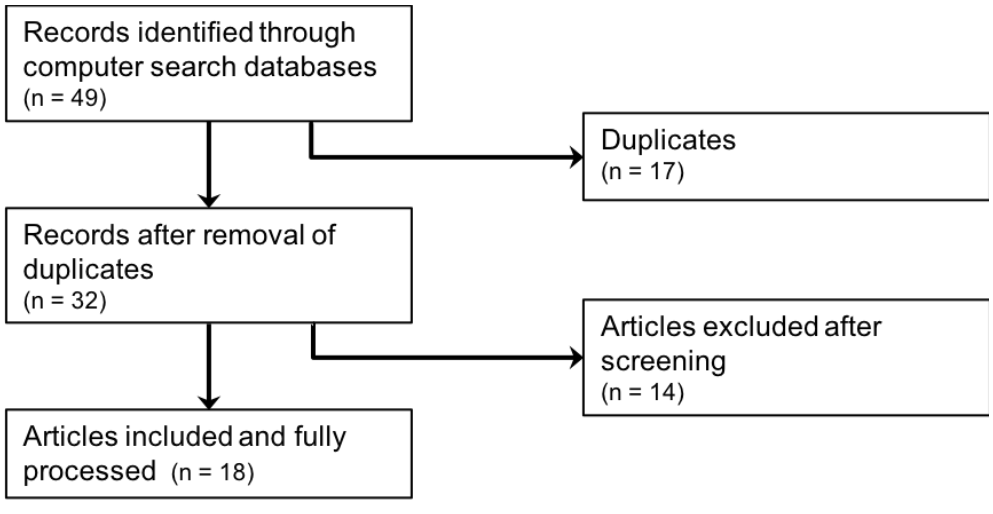

Figure 5.2. Flowchart literature search.

\subsection{Results}

5.4.1. Blood-brain barrier transport and glymphatic clearance as complementary mechanisms

BBB transport and glymphatic clearance have the same purpose in clearing interstitial solutes such as $A \beta$ from the brain and in that sense likely serve complementary roles and seem to be partially overlapping mechanisms (3). As mentioned before, $A \beta$ can be transported across the local BBB by specific efflux transporters such as LRP1 and P-gp $(31,64)$. However, the amount of $A \beta$ exceeds the efflux transporter capacity and often the distance to the $\mathrm{BBB}$ is too large, so that $\mathrm{A} \beta$ must also be cleared through the ISF flow in the glymphatic system (3). The glymphatic system can also stimulate BBB transport as the convective flow spreads $A \beta$ over a wider anatomical area and facilitates $B B B$ transport (Figure 5.3). 


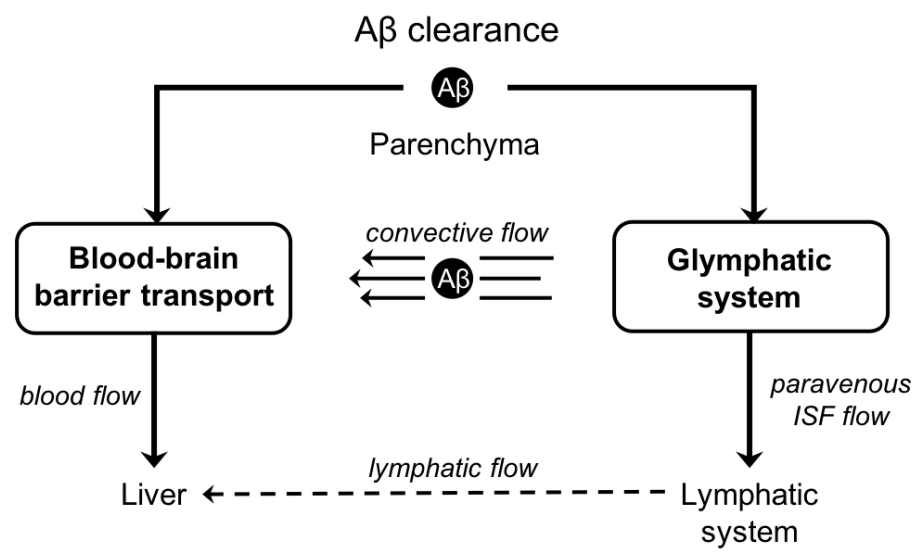

Figure 5.3. The two complementary mechanisms of A $\beta$ clearance through which $A \beta$ eventually leaves the brain. Blood-brain barrier transport of $A \beta$ is further facilitated by convective flow by the glymphatic system moving A $\beta$ toward the transporters.

Cooperation between BBB transport and the glymphatic system becomes evident when studying their mechanics during the sleep-wake cycle. During wakefulness, the interstitial volume is more contracted, which increases resistance to convective flow and CSF movement (65). During sleep, the interstitial space increases in volume, which facilitates convective flow, CSF-to-ISF turnover and thus glymphatic clearance. It is unclear what causes this increase, but the leading hypothesis is that this is due to sleepmediated shrinkage of astroglial cells (66). BBB transport is also partly responsible for the increase in $A \beta$ clearance during sleep $(9,67)$. Convective flow moves $A \beta$ more toward the $\mathrm{BBB}$, so that BBB transport indirectly increases (9). Clearance through BBB transport thus increases by mediation of the glymphatic system.

Defective clearance can already be detected before the onset of neuropathology, which suggests that clearance mechanisms have a causal role in neurodegenerative disorders (68). Optimal functioning of clearance mechanisms could thus delay or even prevent the onset of neurodegenerative disorders. $A \beta$ accumulation is for instance present before any symptoms of $\mathrm{AD}$ manifest themselves. 
Chapter 5

\subsubsection{Glymphatic dysfunction affecting the blood-brain barrier}

The choroid plexus is the organ that secretes CSF. CSF has many functions, like providing buoyancy, nourishment and removal of waste products (69). Formation of the glymphatic circulation is one aspect of the CSF dynamics. Choroid plexus functioning and arterial pulsatility determine the CSF dynamics and for instance regulate CSF influx through the perivascular space and are the driving forces of the glymphatic system $(48,54)$. Factors influencing arterial pulsatility, such as vessel stiffness and heart rate, affect the amount of waste clearance (70). Arterial pulsatility decreases due to processes such as metabolic syndrome, hypertension, hyperlipidemia, diabetes or aging. These processes indirectly affect glymphatic clearance and can possibly lead to neurodegeneration.

Kress et al. (2014) used in vivo and ex vivo fluorescent imaging with a radiotracer in mice to further investigate the relationship between glymphatic clearance, arterial pulsatility and aging. They demonstrated that $A \beta$ clearance through the glymphatic system decreased by 40 percent in the aged brain relative to a younger brain (3). This decrease was associated with a 27 percent decrease in arterial pulsatility. The cerebral arterial wall is enclosed in the pial sheath and the perivascular astrocytic endfeet sheath. Reactive astrogliosis, or changes in the astrocytes due to central nervous system damage, affects the compliance of these structures and impairs arterial pulsatility. Reactive astrogliosis is also associated with a decrease in perivascular AQP4 channel reactivity at the astrocytic endfeet (46). Both impaired arterial pulsatility and changes in perivascular AQP4 channel reactivity can disturb convective flow and lead to glymphatic dysfunction. As a consequence, $A \beta$ accumulation occurs which can further impair perivascular AQP4 channel reactivity (71). Decrease in perivascular AQP4 channel reactivity, glymphatic dysfunction and $A \beta$ accumulation seem to form a vicious feed-forward circle that promotes $A \beta$ accumulation and eventual neurodegeneration (3).

Kyrtsos and Baras (2015) created a computer model simulating how $A \beta$ levels change in the brain parenchyma and vasculature under the influence of glymphatic function and apolipoprotein E allele (70). In this model, glymphatic function was defined by heart rate and vessel stiffness. The model demonstrated that lower heart rate was associated with higher $A \beta$ levels in the brain parenchyma. Greater vessel stiffness also led to higher $A \beta$ levels. Moreover, $A \beta$ level did not only increase in the brain parenchyma, but also in the blood vessel walls as the previously mentioned CAA. Especially carriers of 
the apolipoprotein E4 allele, which is associated with a higher risk of AD (72), demonstrated CAA.

CAA leads to thickening of the basement membrane, luminal narrowing and narrowing of the perivascular space, which blocks the glymphatic clearance route $(47$, 59). CAA also leads to degeneration of smooth muscle cells and endothelial cells $(73,74)$. Endothelial cells contain the BBB transport channels and endothelial degeneration disrupts BBB transport. A study in mice has demonstrated that decreasing the amount of endothelial transporters lead to a 55 percent decrease in $A \beta$ clearance (45). Thus, lower heart rate and greater vessel stiffness affect glymphatic clearance and are associated with cortical $A \beta$ accumulation and CAA. CAA does not only further impair the glymphatic system by perivascular narrowing, but also decreases BBB transport by endothelial degeneration.

\subsubsection{Blood-brain barrier disruption affecting the glymphatic system}

BBB disruption and inflammation are connected in a vicious circle (28). Defensins are peptides that are released as part of the immune response to defend the brain against pathogens (75). Platelet-derived growth factor BB (PDGF-BB) regulates pericyte proliferation and recruitment to ensure microvascular stability. When defensins are expressed on the luminal side of the endothelial membrane, PDGF-BB and defensins compete for the same receptors, which can lead to BBB instability. Moreover, defensins on the luminal side promote the recruitment of mast cells. Mast cells are white blood cells that induce the release of inflammatory mediators leading to BBB disruption. Once in the inflammatory state, reactive oxygen species are released, which affect tight junction integrity and further compromise the BBB (28). Besides BBB disruption, inflammation can also contribute to glymphatic dysfunction. Inflammation slows down the convective flow, decreases CSF-to-ISF turnover and impairs glymphatic clearance (76). On the abluminal side, inadequate defensin expression can occur, which allows activated T-cells, viruses and inflammatory mediators to enter the brain and impair BBB integrity (75). At the same time, glymphatic flow is impeded. Inadequate defensin expression also leads to downregulation of LRP1 and decreased A $\beta$ clearance.

Sleep regulates the immune response and sleep disturbance and inflammation are causally related $(77,78)$. A mouse model of chronic sleep restriction demonstrated that sleep restriction downregulates the expression of tight junction proteins and increases 
Chapter 5

BBB permeability (79). Blood-born substances then freely enter the brain and may trigger excitotoxicity and neuronal death (77). When these pathological processes impair the AQP4 channels, convective flow is impaired and glymphatic dysfunction occurs (4, 11). AQP4 channels are part of the astrocytes, which are important for sleep regulation, as increase in astrocytic gene expression and activation of the astrocytes initiate sleep $(78,80,81)$. To sum up, inflammation often occurs together with sleep disturbance. Inflammation and sleep disturbance can lead to both BBB disruption and glymphatic dysfunction (82). BBB disruption can also lead to glymphatic dysfunction and aggravate sleep disturbance due to its detrimental effect on the astrocytes.

\subsection{Investigating glymphatic function using imaging techniques}

\subsubsection{Practical application of glymphatic function imaging}

For the rat brain, a complete map of glymphatic influx and efflux can be constructed using MRI measurements of tracer distribution over the brain. From this map, the clearance kinetics can be derived (62). According to Nedergaard (2013), it is promising to extend this research approach to humans. Defective clearance could be the initiating factor of AD and the clearance kinetics may be used to identify those at risk of AD or evaluate disease progression (47). Mapping the clearance kinetics could also be used to monitor how a person responds to treatment.

\subsection{2. $\quad$ Progress in glymphatic function imaging techniques}

The first techniques used to image glymphatic function were in vivo 2-photon microscopy and ex vivo fluorescence imaging of intracisternally infused fluorescent CSF tracers (54). However, these techniques are not suitable for clinical practice, due to the complications associated with intracisternal infusion in humans. Previous attempts at cisternal punctures in humans has resulted in acute neurological damage (83). Lumbar intrathecal infusion is a safer and therefore clinically useful infusion method. Yang et al. (2013) compared the clearance kinetics and tracer distribution in rat brain slices after lumbar intrathecal infusion of CSF tracers (FITC-conjugated dextran and Texas Red-conjugated dextran) with the values found after intracisternal infusion (84). The clearance pathway after lumbar intrathecal infusion is consistent with the characterization of the glymphatic system after intracisternal infusion. The researchers concluded that the safer option 
lumbar intrathecal infusion can be used to investigate activity in the glymphatic system instead of the risky intracisternal infusion.

Iliff et al. (2013) used dynamic contrast-enhanced MRI with intrathecal infusion of contrast agents (Magnevist and GadoSpin) in a vital rat brain (62). Contrast agent distribution was used to map the CSF-to-ISF turnover pathway over time and identify the influx nodes and clearance pathways. With this study, Iliff et al. (2013) laid the groundwork for imaging activity in the glymphatic system with a simple technique suited for clinical practice. They viewed their study as the basis for a new strategy to evaluate AD susceptibility and progression.

After Yang et al. (2013) and Iliff et al. (2013) mapped tracer distribution in rodent brains, Eide and Ringstad (2015) applied the same approach to the human brain (85). A woman underwent routine MRI after intrathecal infusion of gadolinium. Gadolinium was widely distributed over the brain and spinal cord, which meant free transport of gadolinium in the CSF and free CSF-to-ISF turnover. Their findings indicated the presence of the glymphatic system, this time in the human brain.

\subsubsection{The future of glymphatic function imaging techniques}

The glymphatic system starts with CSF flowing through the perivascular space. Future attempts to measure activity in the glymphatic system could focus on imaging the CSF flow within the perivascular space.

Recently, Naganawa et al. (2016) detected contrast agent in the human brain with an imaging technique (heavily $\mathrm{T}_{2}$-weighted FLAIR) that is highly sensitive to low gadolinium concentrations in the CSF (86). With this technique, researchers were for the first time able to detect signal enhancement in the perivascular space of the human brain, several hours after gadolinium administration (Figure 5.4 (86)). 

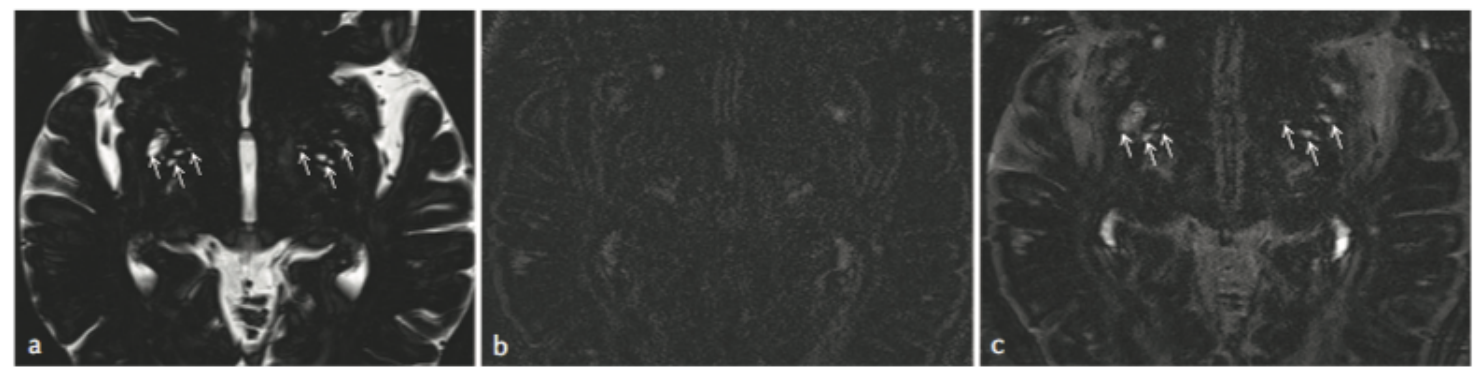

Figure 5.4. a) Magnetic resonance cisternography shows enlarged perivascular spaces in the bilateral basal ganglia (arrows). b) On the pre-contrast heavily $T_{2}$-weighted FLAIR image the perivascular spaces have low signal intensity. c) On the post-contrast heavily $T_{2}$-weighted FLAIR image the perivascular spaces have a high signal intensity. Figure obtained with permission from (86).

The perivascular space is an entrance point to the glymphatic system (70). This new imaging technique to visualize gadolinium in the CSF and perivascular space offers a new way to image the clearance pathway through the glymphatic system in humans (86).

The same research group also introduced a method to measure water diffusivity along the perivascular space in the human brain, called 'diffusion tensor image analysis along the perivascular space' (DTI-ALPS) (87). Lower diffusivity along the perivascular space indicates less activity in the glymphatic system and thus less CSF influx and glymphatic dysfunction. DTI-ALPS in $16 \mathrm{AD}$, nine mild cognitive impairment and six subjective cognitive impairment patients demonstrated a positive correlation between water diffusivity along the perivascular space and Mini-Mental State Examination (MMSE) score. With lower MMSE score and thus more cognitive decline, less activity in the glymphatic system was found. Imaging brain fluid dynamics may help in understanding the pathogenesis of glymphatic dysfunction and early detection of neurodegeneration.

\subsection{Conclusion}

Properly functioning waste clearance systems protect against the accumulation of toxic waste products such as $A \beta$, and may delay or even prevent the onset of neurodegenerative disorders such as AD. Keeping these clearance systems in good condition could be a new focus of future health initiatives. Many studies into waste clearance emphasize the importance of sleep. Sleep disturbance is linked to inflammation, BBB disruption and glymphatic dysfunction. Adhering to a healthy sleep 
pattern and daily physical exercise to stimulate cardiovascular health are relatively easy ways to keep the vasculature healthy and improve waste clearance. Voluntary exercise in aged mice increased waste clearance, decreased $A \beta$ level and promoted synaptic integrity (88). Since there was no association with BBB permeability, it seems likely that the glymphatic system was responsible for the increased clearance. Moreover, mice in the exercise group had better spatial memory, demonstrating that increasing glymphatic clearance by voluntary exercise can improve cognitive functioning.

Techniques to image the glymphatic system continue to be developed, leading to new ways of determining the clearance kinetics. New ways of determining the clearance kinetics may help to identify those at risk of AD or evaluating progression of AD (47). Moreover, if future health interventions are aimed at improving the clearance kinetics (89), imaging the glymphatic system can be a way to monitor response to this type of treatment. Recent advances are focused on imaging CSF flow or water diffusivity in the perivascular space, thereby attempting to visualize activity in the glymphatic system (86, 87). A defective clearance system may be the underlying mechanism of age-related brain pathology and new techniques to determine the efficacy of this clearance system may in the future help in identifying who may be vulnerable to age-related cognitive disorders.

\subsection{Declaration of interest}

The authors declare that they have no competing interests.

\subsection{References}

1. Zlokovic BV. The blood-brain barrier in health and chronic neurodegenerative disorders. Neuron. 2008;57(2):178-201.

2. Groothuis DR, Vavra MW, Schlageter KE, Kang EW, Itskovich AC, Hertzler S, et al. Efflux of drugs and solutes from brain: the interactive roles of diffusional transcapillary transport, bulk flow and capillary transporters. Journal of Cerebral Blood Flow \& Metabolism. 2007;27(1):43-56.

3. Kress BT, Iliff JJ, Xia M, Wang M, Wei HS, Zeppenfeld D, et al. Impairment of paravascular clearance pathways in the aging brain. Annals of neurology. 2014;76(6):845-61.

4. Iliff JJ, Wang M, Liao Y, Plogg BA, Peng W, Gundersen GA, et al. A paravascular pathway facilitates CSF flow through the brain parenchyma and the clearance of interstitial solutes, including amyloid $\beta$. Science translational medicine. 2012;4(147):147ra11-ra11.

5. Wardlaw J. Blood-brain barrier and cerebral small vessel disease. Journal of the neurological sciences. 2010;299(1):66-71. 


\section{Chapter 5}

6. Farrall AJ, Wardlaw JM. Blood-brain barrier: ageing and microvascular disease-systematic review and meta-analysis. Neurobiology of aging. 2009;30(3):337-52.

7. Kalaria RN. Vascular basis for brain degeneration: faltering controls and risk factors for dementia. Nutrition reviews. 2010;68(suppl 2):S74-S87.

8. Burgmans S, van de Haar HJ, Verhey FR, Backes WH. Amyloid- $\beta$ interacts with blood-brain barrier function in dementia: a systematic review. Journal of Alzheimer's Disease. 2013;35(4):859-73.

9. Tarasoff-Conway JM, Carare RO, Osorio RS, Glodzik L, Butler T, Fieremans E, et al. Clearance systems in the brain [mdash] implications for Alzheimer disease. Nature Reviews Neurology. 2015;11(8):457-70.

10. Hawkins RA, Peterson DR, Vina JR. The complementary membranes forming the blood-brain barrier. IUBMB life. 2002;54(3):101-7.

11. Iliff JJ, Chen MJ, Plog BA, Zeppenfeld DM, Soltero M, Yang L, et al. Impairment of Glymphatic Pathway Function Promotes Tau Pathology after Traumatic Brain Injury. The Journal of Neuroscience. 2014;34(49):16180-93.

12. Abbott NJ, Rönnbäck L, Hansson E. Astrocyte-endothelial interactions at the blood-brain barrier. Nature Reviews Neuroscience. 2006;7(1):41-53.

13. Popescu BO, Toescu EC, Popescu LM, Bajenaru O, Muresanu DF, Schultzberg M, et al. Blood-brain barrier alterations in ageing and dementia. Journal of the neurological sciences. 2009;283(1):99-106.

14. Wardlaw J, Sandercock P, Dennis M, Starr J. Is breakdown of the blood-brain barrier responsible for lacunar stroke, leukoaraiosis, and dementia? Stroke. 2003;34(3):806-12.

15. Hawkins BT, Davis TP. The blood-brain barrier/neurovascular unit in health and disease. Pharmacological reviews. 2005;57(2):173-85.

16. Amiry-Moghaddam M, Ottersen OP. The molecular basis of water transport in the brain. Nature reviews Neuroscience. 2003;4(12):991.

17. Nakada T, Kwee IL, Igarashi H, Suzuki Y. Aquaporin-4 Functionality and Virchow-Robin Space Water Dynamics: Physiological Model for Neurovascular Coupling and Glymphatic Flow. International Journal of Molecular Sciences. 2017;18(8):1798.

18. Montagne A, Barnes Samuel R, Sweeney Melanie D, Halliday Matthew R, Sagare Abhay P, Zhao Z, et al. Blood-Brain Barrier Breakdown in the Aging Human Hippocampus. Neuron.85(2):296-302.

19. van de Haar HJ, Burgmans S, Hofman PA, Verhey FR, Jansen JF, Backes WH. Blood-brain barrier impairment in dementia: current and future in vivo assessments. Neuroscience \& Biobehavioral Reviews. 2014.

20. Viggars AP, Wharton SB, Simpson JE, Matthews FE, Brayne C, Savva GM, et al. Alterations in the blood brain barrier in ageing cerebral cortex in relationship to Alzheimer-type pathology: a study in the MRC-CFAS population neuropathology cohort. Neuroscience letters. 2011;505(1):25-30.

21. Zlokovic BV. Clearing amyloid through the blood-brain barrier. Journal of neurochemistry. 2004;89(4):807-11.

22. Haass C, Schlossmacher MG, Hung AY, Vigo-Pelfrey C, Mellon A, Ostaszewski BL, et al. Amyloid $\beta$ peptide is produced by cultured cells during normal metabolism. Nature. 1992;359(6393):322-5. 
23. Deane R, Wu Z, Zlokovic BV. RAGE (Yin) versus LRP (Yang) balance regulates Alzheimer amyloid $\beta$-peptide clearance through transport across the blood-brain barrier. Stroke. 2004;35(11 suppl 1):262831.

24. Hansson SF, Andréasson U, Wall M, Skoog I, Andreasen N, Wallin A, et al. Reduced levels of amyloid$\beta$-binding proteins in cerebrospinal fluid from Alzheimer's disease patients. Journal of Alzheimer's Disease. 2009;16(2):389-97.

25. Zlokovic BV, Martel CL, Matsubara E, McComb JG, Zheng G, McCluskey RT, et al. Glycoprotein 330/megalin: probable role in receptor-mediated transport of apolipoprotein J alone and in a complex with Alzheimer disease amyloid beta at the blood-brain and blood-cerebrospinal fluid barriers. Proceedings of the National Academy of Sciences. 1996;93(9):4229-34.

26. Martel CL, Mackic JB, Matsubara E, Governale S, Miguel C, Miao W, et al. Isoform-Specific Effects of Apolipoproteins E2, E3, and E4 on Cerebral Capillary Sequestration and Blood-Brain Barrier Transport of Circulating Alzheimer's Amyloid $\beta$. Journal of neurochemistry. 1997;69(5):1995-2004.

27. Selkoe DJ. Clearing the brain's amyloid cobwebs. Neuron. 2001;32(2):177-80.

28. Shlosberg D, Benifla M, Kaufer D, Friedman A. Blood-brain barrier breakdown as a therapeutic target in traumatic brain injury. Nature Reviews Neurology. 2010;6(7):393-403.

29. Krohn M, Lange C, Hofrichter J, Scheffler K, Stenzel J, Steffen J, et al. Cerebral amyloid-beta proteostasis is regulated by the membrane transport protein ABCC1 in mice. J Clin Invest. 2011;121(10):3924-31.

30. Cirrito JR, Deane R, Fagan AM, Spinner ML, Parsadanian M, Finn MB, et al. P-glycoprotein deficiency at the blood-brain barrier increases amyloid- $\beta$ deposition in an Alzheimer disease mouse model. Journal of Clinical Investigation. 2005;115(11):3285-90.

31. van Assema DME, Lubberink M, Bauer M, van der Flier WM, Schuit RC, Windhorst AD, et al. Bloodbrain barrier P-glycoprotein function in Alzheimer's disease2012 2012-01-01 00:00:00. 181-9 p.

32. Bartels AL, Kortekaas R, Bart J, Willemsen ATM, de Klerk OL, de Vries JJ, et al. Blood-brain barrier P-glycoprotein function decreases in specific brain regions with aging: A possible role in progressive neurodegeneration. Neurobiology of Aging. 2009;30(11):1818-24.

33. Chiu C, Miller MC, Monahan R, Osgood DP, Stopa EG, Silverberg GD. P-glycoprotein expression and amyloid accumulation in human aging and Alzheimer's disease: preliminary observations. Neurobiology of Aging. 2015;36(9):2475-82.

34. Koldamova R, Fitz NF, Lefterov I. ATP-binding cassette transporter A1: From metabolism to neurodegeneration. Neurobiology of Disease. 2014;72(Part A):13-21.

35. Korczyn AD. The amyloid cascade hypothesis. Alzheimer's \& Dementia. 2008;4(3):176-8.

36. Herrup K. The case for rejecting the amyloid cascade hypothesis. Nature neuroscience. 2015:7949.

37. Kepp KP. Ten Challenges of the Amyloid Hypothesis of Alzheimer's Disease. Journal of Alzheimer's Disease. 2017;55(2):447-57.

38. Behl C. Amyloid in Alzheimer's disease: guilty beyond reasonable doubt? Trends in pharmacological sciences. 2017;38(10):849-51. 


\section{Chapter 5}

39. Echávarri C, Burgmans S, Caballero MC, García-Bragado F, Verhey FR, Uylings H. Co-occurrence of different pathologies in dementia: implications for dementia diagnosis. Journal of Alzheimer's Disease. 2012;30(4):909-17.

40. Wardlaw JM, Smith EE, Biessels GJ, Cordonnier C, Fazekas F, Frayne R, et al. Neuroimaging standards for research into small vessel disease and its contribution to ageing and neurodegeneration. The Lancet Neurology. 2013;12(8):822-38.

41. Jack CR, Knopman DS, Jagust WJ, Shaw LM, Aisen PS, Weiner MW, et al. Hypothetical model of dynamic biomarkers of the Alzheimer's pathological cascade. The Lancet Neurology. 2010;9(1):119-28.

42. Pantoni L. Cerebral small vessel disease: from pathogenesis and clinical characteristics to therapeutic challenges. The Lancet Neurology. 2010;9(7):689-701.

43. Schneider JA, Arvanitakis Z, Bang W, Bennett DA. Mixed brain pathologies account for most dementia cases in community-dwelling older persons. Neurology. 2007;69(24):2197-204.

44. Sironi L, Guerrini U, Tremoli E, Miller I, Gelosa P, Lascialfari A, et al. Analysis of pathological events at the onset of brain damage in stroke-prone rats: A proteomics and magnetic resonance imaging approach. Journal of neuroscience research. 2004;78(1):115-22.

45. Shibata M, Yamada S, Kumar SR, Calero M, Bading J, Frangione B, et al. Clearance of Alzheimer's amyloid- $\beta$ 1-40 peptide from brain by LDL receptor-related protein-1 at the blood-brain barrier. The Journal of clinical investigation. 2000;106(12):1489-99.

46. Iliff JJ, Nedergaard M. Is there a cerebral lymphatic system? Stroke. 2013;44(6 suppl 1):S93-S5.

47. Nedergaard M. Garbage truck of the brain. Science. 2013;340(6140):1529-30.

48. Hladky SB, Barrand MA. Mechanisms of fluid movement into, through and out of the brain: evaluation of the evidence. Fluids and Barriers of the CNS. 2014;11(1):1.

49. Louveau A, Smirnov I, Keyes TJ, Eccles JD, Rouhani SJ, Peske JD, et al. Structural and functional features of central nervous system lymphatic vessels. Nature. 2015;523(7560):337-41.

50. Ma Q, Ineichen BV, Detmar M, Proulx ST. Outflow of cerebrospinal fluid is predominantly through lymphatic vessels and is reduced in aged mice. Nature Communications. 2017;8(1):1434.

51. Jessen NA, Munk ASF, Lundgaard I, Nedergaard M. The glymphatic system: a beginner's guide. Neurochemical research. 2015;40(12):2583-99.

52. Xu Z, Xiao N, Chen Y, Huang H, Marshall C, Gao J, et al. Deletion of aquaporin-4 in APP/PS1 mice exacerbates brain $A \beta$ accumulation and memory deficits. Molecular neurodegeneration. 2015;10(1):58.

53. Zeppenfeld DM, Simon M, Haswell JD, D’abreo D, Murchison C, Quinn JF, et al. Association of perivascular localization of aquaporin-4 with cognition and Alzheimer disease in aging brains. JAMA neurology. 2017;74(1):91-9.

54. Iliff JJ, Wang M, Zeppenfeld DM, Venkataraman A, Plog BA, Liao Y, et al. Cerebral arterial pulsation drives paravascular CSF-interstitial fluid exchange in the murine brain. The Journal of Neuroscience. 2013;33(46):18190-9.

55. Jiang Q, Zhang L, Ding G, Davoodi-Bojd E, Li Q, Li L, et al. Impairment of the glymphatic system after diabetes. Journal of Cerebral Blood Flow \& Metabolism. 2016:0271678X16654702.

56. Mestre H, Kostrikov S, Mehta RI, Nedergaard M. Perivascular spaces, glymphatic dysfunction, and small vessel disease. Clinical Science. 2017;131(17):2257-74. 
57. Wang M, Ding F, Deng S, Guo X, Wang W, Iliff JJ, et al. Focal solute trapping and global glymphatic pathway impairment in a murine model of multiple microinfarcts. Journal of Neuroscience. 2017;37(11):2870-7.

58. Cirrito JR, Yamada KA, Finn MB, Sloviter RS, Bales KR, May PC, et al. Synaptic activity regulates interstitial fluid amyloid- $\beta$ levels in vivo. Neuron. 2005;48(6):913-22.

59. Roher AE, Kuo Y-M, Esh C, Knebel C, Weiss N, Kalback W, et al. Cortical and leptomeningeal cerebrovascular amyloid and white matter pathology in Alzheimer's disease. Molecular Medicine. 2003;9(3-4):112.

60. Wostyn P, Van Dam D, Audenaert K, Killer HE, De Deyn PP, De Groot V. A new glaucoma hypothesis: a role of glymphatic system dysfunction. Fluids and Barriers of the CNS. 2015;12(1):1.

61. Venkat P, Chopp M, Zacharek A, Cui C, Zhang L, Li Q, et al. White matter damage and glymphatic dysfunction in a model of vascular dementia in rats with no prior vascular pathologies. Neurobiology of aging. 2017;50:96-106.

62. Iliff JJ, Lee H, Yu M, Feng T, Logan J, Nedergaard M, et al. Brain-wide pathway for waste clearance captured by contrast-enhanced MRI. The Journal of clinical investigation. 2013;123(3):1299-309.

63. Grant MJ, Booth A. A typology of reviews: an analysis of 14 review types and associated methodologies. Health Information \& Libraries Journal. 2009;26(2):91-108.

64. Zuroff L, Daley D, Black KL, Koronyo-Hamaoui M. Clearance of cerebral Abeta in Alzheimer's disease: reassessing the role of microglia and monocytes. Cellular and molecular life sciences : CMLS. 2017. 65. Xie L, Kang H, Xu Q, Chen MJ, Liao Y, Thiyagarajan M, et al. Sleep drives metabolite clearance from the adult brain. science. 2013;342(6156):373-7.

66. Mendelsohn AR, Larrick JW. Sleep facilitates clearance of metabolites from the brain: glymphatic function in aging and neurodegenerative diseases. Rejuvenation research. 2013;16(6):518-23.

67. Hladky SB, Barrand MA. Metabolite Clearance During Wakefulness and Sleep. Handbook of experimental pharmacology. 2017.

68. Peng W, Achariyar TM, Li B, Liao Y, Mestre H, Hitomi E, et al. Suppression of glymphatic fluid transport in a mouse model of Alzheimer's disease. Neurobiology of disease. 2016;93:215-25.

69. Spector R, Robert Snodgrass S, Johanson CE. A balanced view of the cerebrospinal fluid composition and functions: Focus on adult humans. Experimental Neurology. 2015;273:57-68.

70. Kyrtsos CR, Baras JS. Modeling the Role of the Glymphatic Pathway and Cerebral Blood Vessel Properties in Alzheimer's Disease Pathogenesis. PloS one. 2015;10(10):e0139574.

71. Yang J, Lunde LK, Nuntagij P, Oguchi T, Camassa L, Nilsson LN, et al. Loss of astrocyte polarization in the tg-ArcSwe mouse model of Alzheimer's disease. Journal of Alzheimer's Disease. 2011;27(4):711-22. 72. Saunders AM, Strittmatter WJ, Schmechel D, George-Hyslop PS, Pericak-Vance M, Joo S, et al. Association of apolipoprotein E allele $\epsilon 4$ with late-onset familial and sporadic Alzheimer's disease. Neurology. 1993;43(8):1467-.

73. Saito S, Ihara M. Interaction between cerebrovascular disease and Alzheimer pathology. Current opinion in psychiatry. 2016;29(2):168-73. 


\section{Chapter 5}

74. Hawkes CA, Sullivan PM, Hands S, Weller RO, Nicoll JA, Carare RO. Disruption of arterial perivascular drainage of amyloid- $\beta$ from the brains of mice expressing the human APOE $\varepsilon 4$ allele. PloS one. 2012;7(7):e41636.

75. Kazakos EI, Kountouras J, Polyzos SA, Deretzi G. Novel aspects of defensins' involvement in virusinduced autoimmunity in the central nervous system. Medical Hypotheses. 2017;102(Supplement C):33-6. 76. Erickson MA, Hartvigson PE, Morofuji Y, Owen JB, Butterfield DA, Banks WA. Lipopolysaccharide impairs amyloid beta efflux from brain: altered vascular sequestration, cerebrospinal fluid reabsorption, peripheral clearance and transporter function at the blood-brain barrier. Journal of neuroinflammation. 2012;9(1):150.

77. He J, Hsuchou H, He Y, Kastin AJ, Wang Y, Pan W. Sleep restriction impairs blood-brain barrier function. The Journal of Neuroscience. 2014;34(44):14697-706.

78. Aguirre CC. Sleep deprivation: A mind-body approach. Current Opinion in Pulmonary Medicine. 2016;22(6):583-8.

79. Pan W, Kastin AJ. The Blood-Brain Barrier Regulatory Roles in Wakefulness and Sleep. The Neuroscientist. 2016:1073858416639005.

80. Bellesi M, de Vivo L, Tononi G, Cirelli C. Effects of sleep and wake on astrocytes: clues from molecular and ultrastructural studies. BMC biology. 2015;13(1):66.

81. Pelluru D, Konadhode RR, Bhat NR, Shiromani PJ. Optogenetic stimulation of astrocytes in the posterior hypothalamus increases sleep at night in C57BL/6J mice. European Journal of Neuroscience. 2015.

82. Harding. Can Better Management of Periodontal Disease Delay the Onset and Progression of Alzheimer's Disease? Journal of Alzheimer's Disease. 2017;58(2):337-48.

83. Keane JR. Cisternal puncture complications-treatment of coccidioidal meningitis with amphotericin B. California medicine. 1973;119(3):10.

84. Yang L, Kress BT, Weber HJ, Thiyagarajan M, Wang B, Deane R, et al. Evaluating glymphatic pathway function utilizing clinically relevant intrathecal infusion of CSF tracer. Journal of translational medicine. 2013;11(1):107.

85. Eide PK, Ringstad G. MRI with intrathecal MRI gadolinium contrast medium administration: a possible method to assess glymphatic function in human brain. Acta radiologica open. 2015;4(11):2058460115609635.

86. Naganawa S, Nakane T, Kawai H, Taoka T. Gd-based contrast enhancement of the perivascular spaces in the basal ganglia. Magnetic Resonance in Medical Sciences. 2017;16(1):61-5.

87. Taoka T, Masutani Y, Kawai H, Nakane T, Matsuoka K, Yasuno F, et al. Evaluation of glymphatic system activity with the diffusion MR technique: diffusion tensor image analysis along the perivascular space (DTI-ALPS) in Alzheimer's disease cases. Japanese journal of radiology. 2017;35(4):172-8.

88. He X-f, Liu D-x, Zhang Q, Liang F-y, Dai G-y, Zeng J-s, et al. Voluntary exercise promotes glymphatic clearance of amyloid beta and reduces the activation of astrocytes and microglia in aged mice. Frontiers in molecular neuroscience. 2017;10. 
Interaction between blood-brain barrier and glymphatic system in solute clearance

89. Ren H, Luo C, Feng Y, Yao X, Shi Z, Liang F, et al. Omega-3 polyunsaturated fatty acids promote amyloid- $\beta$ clearance from the brain through mediating the function of the glymphatic system. The FASEB Journal. 2017;31(1):282-93. 

Chapter 6

\subsection{Abstract}

Choroid plexus epithelial cells form a barrier that enables active, bidirectional exchange between the blood plasma and cerebrospinal fluid (CSF), known as the blood-CSF barrier (BCSFB). Through its involvement in CSF composition, the BCSFB maintains homeostasis in the central nervous system. While the relation between blood-brain barrier disruption, aging and neurodegeneration is extensively studied using contrast-enhanced MRI, applying this technique to investigate BCSFB disruption in age-related neurodegeneration has received little attention. This review provides an overview of the current status of contrast-enhanced MRI to assess BCSFB permeability. Post-contrast ventricular gadolinium enhancement has been used to indicate BCSFB permeability. Moreover, new techniques highly sensitive to low gadolinium concentrations in the CSF, for instance heavily T2-weighted imaging with cerebrospinal fluid suppression, seem promising. Also, attempts are made at using other contrast agents, such as manganese ions or very small superparamagnetic iron oxide particles, that seem to be cleared from the brain at the choroid plexus. Advancing and applying new developments such as these could progress the assessment of BCSFB integrity.

\section{Keywords}

Blood-cerebrospinal fluid barrier, choroid plexus, MRI, contrast-enhanced, aging

\subsection{Introduction}

Over the last years, there has been great interest in studies investigating the association between blood-brain barrier (BBB) disruption, aging and neurodegeneration (1-4). The most important function of the BBB involves maintaining homeostasis in the central nervous system (CNS) by the regulated exchange of valuable biomolecules, blocking the entrance of pathogens and clearing waste products from the internal milieu. This function is actually shared by the choroid plexus epithelial cells, that control the exchange between the blood and the cerebrospinal fluid (CSF), and thereby form the blood-CSF barrier (BCSFB) (5). The finding that age-associated damage to the barriers can disrupt balance in the internal milieu and contribute to the neurodegenerative cascade, applies to both the BBB and BCSFB. The role of the BCSFB in the production of CSF has been appreciated for a long time, while its function in maintaining homeostasis in the CNS has 
often been overlooked (6). Investigating BCSFB disruption, and its possible connection to aging and neurodegeneration, could shed more light on age-related decline.

Broadening the focus from only the BBB to also investigating BCSFB disruption raises the question of what methods can be used to accurately measure BCSFB permeability. For BBB disruption, measuring the spread of a contrast agent from the vasculature to the brain with MRI has produced new insights, for instance the detection of subtle BBB leakage in neurodegenerative disorders (7). Therefore, it would be interesting to investigate whether contrast enhancement on MRI could also be used to obtain information on BCSFB permeability, and if so, how this technique should be implemented. To address these points, we conducted a literature review to obtain an overview of what has already been investigated on BCSFB permeability using contrastenhanced MRI in both animal and human studies. From this overview, we attempted to outline what techniques hold most promise for the future of assessing BCSFB integrity.

\subsubsection{Normal function of the BCSFB}

The BCSFB is formed by choroid plexus epithelial cells. The choroid plexus is located at the inner ventricular surface of the lateral ventricles and at the roof of the third and fourth ventricle (Figure 1A) $(8,9)$. The blood vessels in the choroid plexus are fenestrated, and choroid plexus epithelial cells connected by tight junctions surround the fenestrated capillaries to form a barrier for the molecules that leak from these capillaries (Figure 1B) $(5,10)$. 


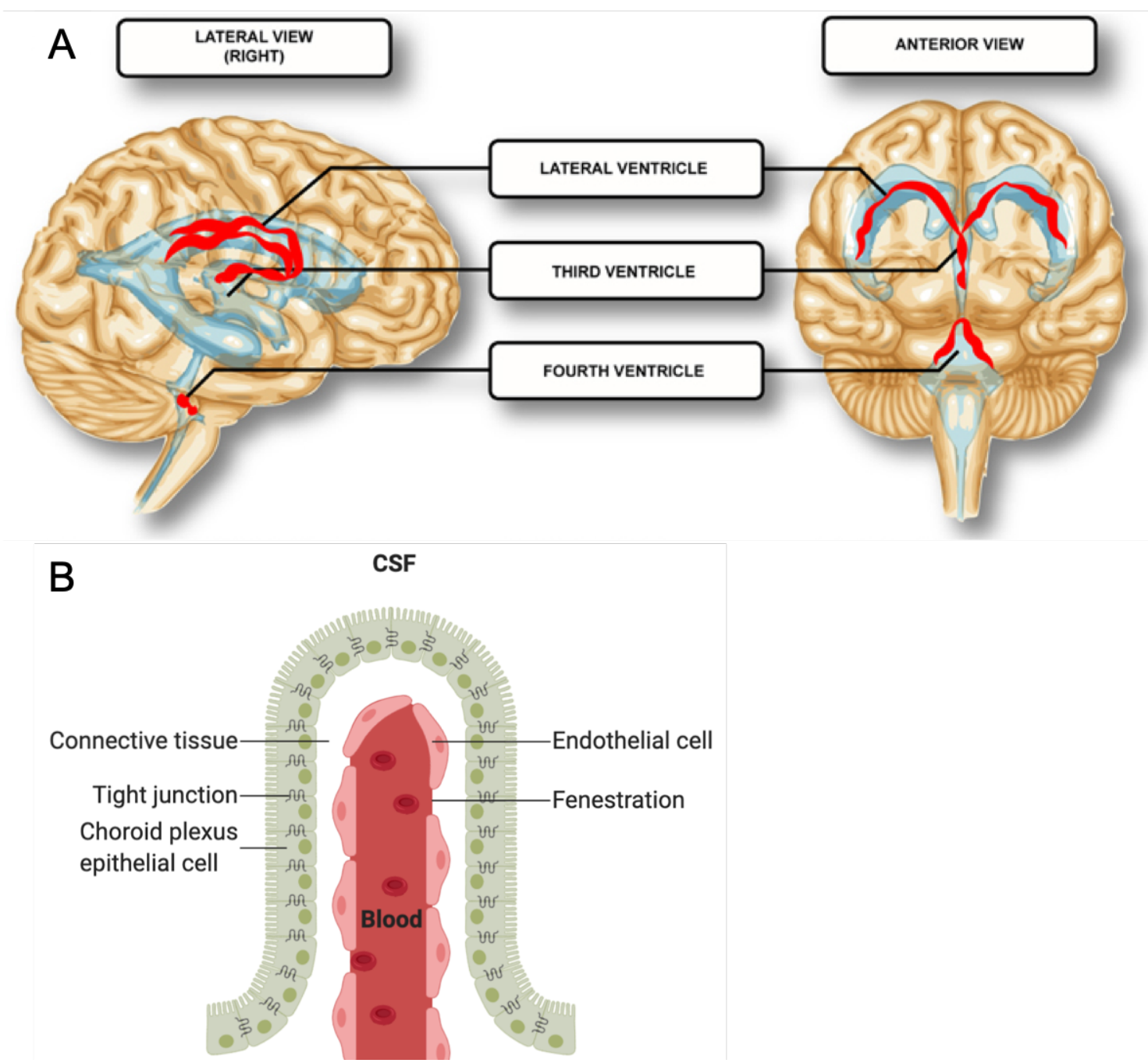

Figure 6.1. The choroid plexus. A: The location of the choroid plexus (red ribbons) in the lateral ventricles, and third and fourth ventricle. Figure obtained from (8), under the Creative Commons Attribution 4.0 License (CC BY 4.0). B: Schematic overview on the cellular level of the choroid plexus forming the blood-CSF barrier. Figure created with BioRender.com.

The choroid plexus is considered the most important structure in the production of CSF (11). Driven by the pressure gradient between blood and ventricles, blood plasma is filtered by the choroid plexus fenestrated capillaries and then transported across the choroid plexus epithelial cells to become part of the CSF (12). An alternative theory opposes the classical view that CSF production is located in a single organ, and instead states that the CSF is produced and absorbed throughout the whole circulatory system at the perivascular spaces $(13,14)$. However, this theory does not account for the ebbs and flows of CSF or the net flow $(11,15)$, and needs independent confirmation. Moreover, a number of reviews have since refuted this theory in favour of evidence of secretion by 
the choroid plexus (16-18), which is more generally accepted. Therefore, in this review, we will focus on fluid exchange at the level of the choroid plexus.

Besides functioning as a barrier, the choroid plexus epithelial cells enable active, bidirectional exchange between the blood and CSF (5). CSF has a different composition than blood plasma. The concentrations of $\mathrm{Cl}^{-}$and $\mathrm{Mg}^{2+}$ ions are higher in the CSF, and the concentrations of $\mathrm{Na}^{+}, \mathrm{K}^{+}$and $\mathrm{Ca}^{2+}$ ions are lower in the CSF compared to the blood plasma (19). Moreover, the concentrations of glucose and amino acids in the CSF are approximately half of that in the blood plasma. These differences indicate that CSF is not just the result of blood plasma filtration by the choroid plexus (20).

The BCSFB contains several transporters involved in the clearance of the neurotoxic amyloid- $\beta$ protein $(A \beta)$, known for its role in the pathogenesis of Alzheimer's disease (AD), such as lipoprotein receptor-related protein 1 and 2 (LRP-1 and LRP-2), receptors for advanced glycation end products (RAGE) and p-glycoprotein (p-gp). The BCSFB also secretes the protein transthyretin (TTR), which, just like the transporters, binds soluble $A \beta$ to facilitate its clearance from the brain, and prevents the formation of $\mathrm{A} \beta$ plaques $(10,21)$.

Other BCSFB transporters supply nutrients and neurotrophic factors to the CSF, and the CSF facilitates their distribution over the whole CNS. Neurotrophic factors are produced at the BCSFB, such as glial cell-derived neurotrophic factor (GDNF), brainderived neurotrophic factor (BDNF) and nerve growth factor (NGF) (22). Moreover, the BCSFB plays a role in the immune response, as the choroid plexus epithelial cells can trigger an inflammatory response by the production of chemokines and cytokines, and also as T-lymphocytes can enter the brain through the BCSFB (10).

\subsubsection{The blood-cerebrospinal fluid barrier in aging and neurodegeneration}

The BCSFB undergoes structural and functional changes during normal aging (10). The structural changes involve atrophy of the choroid plexus epithelial cells, evident by a decrease in height and thickening of the basement membrane (23). Also, neurotoxic proteins accumulate in the choroid plexus epithelial cells $(10,24)$, such as Biondi bodies and lipofuscin deposits, that alter cell function (25). A possible functional change is a decrease in CSF secretion, that has been well established in animal studies (26-28). However, in human studies, the results are less clear. One study found that the elderly had significantly lower CSF production compared to the young (29), but this study used 
a modified Masserman method, which has been criticized as being unusable with different CSF volumes (30). Moreover, another study did not find a significant difference in CSF production between an older and a younger group (31). CSF volume increases during aging due to cerebral atrophy (32), which could lead to decreased CSF turnover, especially when combined with decreased CSF production (24).

The structural and functional changes in the BCSFB during normal aging are exacerbated in $\operatorname{AD}(10,24)$. Choroid plexus epithelial cells, for instance, show more shrinkage and increased thickening of the basement membrane (33). Also, a stronger decrease in CSF production has been found in $\mathrm{AD}(34,35)$, but again the Masserman Technique has been used, which could give unreliable results with different CSF volumes (30). However, a large increase in CSF volume in AD (36), could still lead to a large decrease in CSF turnover, which promotes the glycation of proteins and increases the amount of advanced glycation end products (AGEs), that are prone to aggregation and trigger age-related pathology (37).

The increase of amyloid protein oligomers in the brain is due to increased production and decreased clearance of $A \beta$, combined with decreased CSF turnover and increased protein glycation $(24,38)$. The BCSFB contains transporters to take up A $\beta$ for degradation, but AD impairs the choroid plexus epithelial cell lysosomal function (10). Rather than being cleared, $A \beta$ accumulates in the choroid plexus epithelial cells, which can lead to oxidative stress and cell death and increase BCSFB disruption (39). Moreover, the BCSFB transporters that normally clear A $\beta$ from the CSF to the blood are compromised in $\mathrm{AD}$, which can lead to extracellular $\mathrm{A} \beta$ accumulation at the apical membrane, which can then further increase BCSFB disruption $(39,40)$. This can impair the ability of the BCSFB to secrete $A \beta$-binding proteins, that normally interact with $A \beta$ to facilitate its clearance $(40,41)$. One of these proteins is for instance TTR, which is synthesized and secreted by the choroid plexus, and normally binds and stabilizes soluble $A \beta$ to facilitate its clearance and prevent the formation of $A \beta$ plaques $(42,43)$. A decrease in TTR level, which has been found in $A D$, can therefore lead to more $A \beta$ accumulation in the brain $(21,44)$.

Transport of folate and vitamin B12 from the blood to the CSF through the choroid plexus is also decreased in $\operatorname{AD}(45,46)$. Folate and vitamin $\mathrm{B} 12$ catalyze methylation, which means adding a methyl group to a substrate, and this process is for instance important for the formation of neurotransmitters, myelin and the regulation of $A \beta$ levels 
(47). Lower levels in the CSF, in combination with a decrease in CSF turnover, can lead to less folate and vitamin B12 being distributed over and supplied to the brain (24). Decreased expression of tight junctions on the choroid plexus epithelial cells has also been found in $\mathrm{AD}$ (48). These changes are associated with increased epithelial leakage, which can in turn affect CNS homeostasis and aggravate neurodegenerative processes.

\subsubsection{The immune response in the choroid plexus during aging}

Immune cell trafficking into the CNS increases with age (49). An important component of the immune response are T-lymphocytes recognizing antigens in the CNS, which is important to maintain the integrity of the CNS, and support neurogenesis, neurotrophic factor production, and hippocampus-dependent learning and memory $(50,51)$. Tlymphocytes accumulate at the choroid plexus (52). As the choroid plexus is involved in the communication between the blood and CSF, T-lymphocytes at the choroid plexus can react to antigens in the $\mathrm{CSF}$, and subsequently release cytokines that influence the choroid plexus epithelial cells and the CNS (53). The choroid plexus was demonstrated to contain CNS-specific CD4+ memory cells, which contain receptors for T-lymphocytes that specifically react to antigens in the CNS, and mainly secrete the cytokine interleukin-4 (IL-4), which is involved in neuroprotection (54).

During aging, T-lymphocytes in the choroid plexus maintain their CNS specificity, but the response shifts towards the T-helper type 2 (Th2) cells (54). The Th2 response increases the amount of IL-4 that is released into the CSF (55). However, overexpression of IL-4 actually has a detrimental effect on CNS homeostasis, and consequently, brain functioning. Low levels of IL-4 would normally induce BDNF production, which is beneficial for CNS homeostasis, while high levels of IL-4 lead to BDNF dysregulation. Also, high levels of IL-4 induce the production of C-C motif chemokine 11 (CCL11) which suppresses IL-4 and interferes with IL-4 signaling (56). It has been demonstrated that the immune response mediated by T-lymphocytes is important for hippocampus-related cognitive functions $(50,57)$, and it was suggested that dysregulation of T-lymphocyte signaling at the choroid plexus epithelial cells could be a cause of age-related cognitive decline (54). 
Chapter 6

6.2.4. The relevance of the development of imaging techniques for the central nervous system barriers

Homeostasis in the CNS is dependent on the dynamics between the neurofluids, a collective name for all fluids inside the CNS, namely the blood, CSF and ISF (58). Several diseases have been associated with a disturbance in neurofluid dynamics, such as sleep disorders (59), AD (60), Parkinson's disease (61), traumatic brain injury (62) and stroke (63). A disturbance in neurofluid dynamics can be caused by BBB disruption, BCSFB disruption and/or glymphatic dysfunction. The glymphatic system is a waste clearance system using the microcirculation of the CSF. Some publications report that CSF is exchanged with ISF due to convective flow from the para-arterial to the paravenous spaces, to clear solutes from the interstitial space $(64,65)$. Recently, however, other researchers claim that the evidence for convective flow in the brain parenchyma is lacking $(66,67)$. It has been proposed that convective flow in the para-arterial spaces allows CSF to enter the brain parenchyma, but CSF moves through the interstitial space and exchanges with ISF through diffusion rather than convective flow (67). Diseases in which BBB disruption, BCSFB disruption and/or glymphatic dysfunction, and thus disturbance in neurofluid dynamics, are involved can be grouped under the term CNS interstitial fluidopathies (68). The development of dedicated imaging techniques to obtain specific information on the dynamics between neurofluids may help in understanding the underlying pathophysiology of CNS interstitial fluidopathies, and might even help in the development of novel common treatment options.

\subsection{5. $\quad$ Assessing barrier permeability}

Studies into BCSFB disruption mostly use the CSF/serum albumin ratio as an index of BCSFB integrity, in which the level of the blood-specific protein albumin is measured in the CSF by lumbar puncture and in the serum by venipuncture (69-72). For the in vivo assessment of BBB disruption, however, an increasingly used method is dynamic contrast-enhanced (DCE) MRI, in which a gadolinium-based contrast agent (GBCA) is intravenously injected during scanning. The transfer of the contrast from the blood plasma into the brain tissue is measured over time, after which pharmacokinetic modelling is used to separate the blood component from the tissue component and calculate the leakage rate (73). Unlike the CSF/serum albumin ratio, DCE MRI can 
measure very subtle leakage in a specific brain region, and can therefore separate BBB disruption from BCSFB disruption (74).

The detection of contrast enhancement on MRI to calculate BBB leakage has produced interesting results and provided new insights into the role of BBB disruption in aging and neurodegeneration $(74,75)$. This approach may also be useful for calculating BCSFB leakage, but this has been given less attention. Therefore, this paper explores how contrast enhancement on MRI can be used to assess BCSFB permeability. For this purpose, we conducted a literature review to make an inventory of methods using contrast enhancement on MRI for BCSFB permeability assessment, and to summarize the findings of the available studies.

\subsection{Methods}

To explore how contrast enhancement on MRI is being used to assess BCSFB permeability, a literature search was conducted in the PubMed database. The search terms blood-cerebrospinal fluid barrier (using MeSH terminology and the synonyms blood-cerebrospinal fluid, blood-CSF barrier, blood-CSF, BCSFB, BCB, or choroid plexus), contrast agent (using MeSH terminology and the synonyms contrast, contrast-enhanced, or gadolinium) and MRI (using MeSH terminology and the synonyms magnetic resonance imaging, imaging, or MR imaging) were used. Papers from the years 2000 up to and including 2020 written in English were included in the search. The search produced in total 254 results.

All 254 papers were screened using the title and abstract to check whether they actually applied contrast-enhanced MRI to assess BCSFB function. Papers were included when they met the following inclusion criteria: 1) reporting an original study. 2) combining MRI with administration of some type of contrast agent. 3) Investigating BCSFB permeability by assessing contrast enhancement dynamics at the choroid plexus and/or ventricular system.

After this screening, 20 papers remained. These papers were fully read and crossreferencing was used. From each study, we extracted the following information: sample, age of participants in human studies, MRI technique, contrast agent type and dosage, injection method, imaging delay time after contrast injection and indicator of BCSFB permeability. In the sections below, we describe the main findings of each study to give a 
Chapter 6

complete summary of what has already been done and what can already be concluded regarding the use of contrast enhancement on MRI to assess BCSFB permeability.

\section{4. $\quad$ Results \\ 6.4.1. Animal studies}

See Table 6.1 for a summary of the included animal studies.

\subsubsection{Gadolinium enhancement in the ventricles}

Nixon et al. (2008) used post-contrast T1-weighted MRI immediately after intravenous administration of a GBCA and assessed the hyperintense volume in the ventricles. Hyperintense areas were manually defined and the intensities of those pixels summed. The authors demonstrated that the GBCA was evenly distributed throughout the ventricles of healthy rats given ethanol and thiamine-depleted rats given glucose (76). They conclude that GBCA must have entered the ventricles through the choroid plexus, and impairment in the choroid plexus seems to be an early event in ethanol intoxication and alcoholism.

Batra et al. (2010) used fluid-attenuated inversion recovery (FLAIR) before and 10 minutes after intravenous administration of a GBCA in rats that underwent unilateral middle cerebral artery occlusion (MCAO) or sham surgery. Contrast injection and imaging were performed 1 hour, 24 hours or 48 hours after reperfusion. BCSFB permeability was measured using the change in signal intensity in the ventricles from the pre-contrast to post-contrast images, with normalization by pre-contrast values. Change in signal intensity in the ventricles of the MCAO rats was compared to the intensity change in the sham rats. The 1-hour, 24-hour and 48-hour groups had significantly more gadolinium enhancement than the sham group. The authors also investigated whether the matrix metalloproteinases 2 and 9 (MMP-2 and MMP-9), which are often found after a stroke (77), are associated with BBB and BCSFB disruption (78). Plasma MMP-9 levels were indeed significantly correlated with gadolinium enhancement in the ventricles in the 1-hour group, so plasma MMP-9 could be indicative of acute BCSFB disruption. The authors emphasize the research opportunities of early BCSFB disruption, and the possibility of combining imaging markers such as post-contrast FLAIR gadolinium enhancement with blood biomarkers. 
Saito et al. (2011) investigated whether changes in BBB and BCSFB integrity in prenatally irradiated rats could be detected using pre- and post-contrast T1-weighted MRI with intravenous administration of a GBCA and perfusion MRI (79). Signal intensity after GBCA administration was divided by signal intensity before GBCA administration to obtain the gadolinium enhancement ratio. No significant enhancement ratio was found in the ventricles after administration of GBCA, but perfusion MRI did demonstrate decreased cerebral blood flow, which the authors attributed to abnormal capillaries and arteries. These results were explained as the prenatally irradiated rats having a normally formed BBB and BCSFB, but immature capillary development.

Ichikawa et al. (2011) used T1-weighted MRI before and 5 minutes after intravenous administration of a GBCA, to investigate early changes in CNS barrier permeability in rats with induced meningitis (80). The gadolinium enhancement ratio was calculated by the change in signal intensity due to the GBCA relative to the native signal intensity (81). However, while meningitis is associated with BBB disruption (82), the authors did not observe a significant gadolinium enhancement ratio in the lateral ventricles indicative of BCSFB disruption. A significant enhancement ratio was found in the subarachnoid space, indicating that GBCAs could cross the arachnoid membrane (80).

Yang et al. (2019) investigated whether the multiple PDZ-domain (MPDZ) gene is important for the integrity of the BCSFB (83). The MPDZ protein is located close to the tight junctions of the choroid plexus epithelial cells and mutations in the MPDZ gene have been associated with hydrocephalus, which involves impairment in CSF circulation and causes CSF to accumulate in the ventricles (84). The authors used T1-weighted MRI immediately after intravenous administration of a GBCA (83) in normal mice and MPDZknockout mice (85). The gadolinium enhancement ratio was calculated as the total intensity divided by the difference between the maximum and minimum intensity. Only in the MPDZ-knockout mice, significant gadolinium enhancement was found in the ventricles, and this GBCA leakage was attributed to choroid plexus epithelial cell deficits, demonstrating that the MPDZ gene is indeed important for choroid plexus integrity and function.

\subsubsection{New techniques}

Jost et al. (2016) used gadolinium enhancement to investigate how substances can infiltrate the CSF in healthy rats, so in the absence of a disorder or genetic deficit 
Chapter 6

impairing BCSFB integrity. Therefore, a more sensitive method to measure more subtle leakage was needed (86). Heavily T2-weighted fluid-attenuated inversion recovery (hT2w-FLAIR) was applied before and 1 minute after intravenous administration of a GBCA, as this technique is especially sensitive to detect low concentrations of GBCA within the CSF $(87,88)$. The authors compared intravenous administration of three linear and two macrocyclic GBCAs (86). On T1-weighted images, no gadolinium enhancement could be detected, but with hT2w-FLAIR, qualitative evaluation demonstrated that signal intensity in the CSF spaces (arachnoid space, cerebral aqueduct and inner auditory canal) increased after GBCA administration for each type of GBCA.

Jost et al. (2016) noted that they may not have included the time point of maximal enhancement, and could not determine the precise location of infiltration from GBCAs into the brain (86). Therefore, in a subsequent study, Jost et al. (2017) elaborated on their previous study by investigating whether the blood-CSF pathway could be an alternative route for GBCAs to enter the brain (89). HT2w-FLAIR MRI was performed in healthy rats before and 1 minute after intravenous administration, with temporal evaluations at 9 and 25 minutes after administration, and again 4 hours after administration. Signal intensity in the CSF cavities (third and fourth ventricles, subarachnoid space and cerebral aqueduct) increased significantly from pre-contrast to post-contrast images. Signal intensity increased at the fastest rate in the ventricles and cerebral aqueduct, which supports the idea that GBCAs enter the CNS through the choroid plexus.

By modelling the time course of the spread of a GBCA with DCE MRI, BBB leakage in a single voxel can potentially be calculated, but this requires modelling assumptions (90). Nathoo et al. (2016) developed a novel, voxel-based analysis technique to visualize BBB leakage at the level of a single voxel, that does not require any modelling assumption (91). With this technique, the average signal intensity of a series of pre-contrast T1weighted images before intravenous administration of a GBCA and a series of postcontrast T1-weighted images was calculated, and statistically compared. This technique would prevent selection of an arbitrary ROI, which could be beneficial for investigating processes such as hypoxia that lead to nonspecific and diffuse BBB disruption (92). Though the aim was to assess BBB disruption, the application of this technique in rats with induced cortical injury, hypoxia or lipopolysaccharide-associated inflammation, demonstrated major BCSFB disruption in hypoxia and inflammation, with increased GBCA transfer into the ventricles and periventricular enhancement (91). 


\subsubsection{Other contrast agents}

In MRI studies, gadolinium compounds are most frequently used as contrast agent, but other contrast agents are available. Manganese ions, for instance, demonstrate T1shortening in tissues and appear hyperintense on post-contrast T1-weighted images (93). While gadolinium has a short window of enhancement, manganese enhancement persists beyond this window. Post-contrast manganese-enhanced imaging always takes place at least 30 minutes after contrast administration. Aoki et al. (2004) used T1weighted MRI before and at various time intervals after intravenous administration of a manganese chloride solution in healthy rats (94). The authors demonstrated increased signal intensity compared to the pre-contrast images in the choroid plexus 10 minutes after administration, followed by the ventricles and circumventricular organs (CVOs).

In the field of experimental biomedical imaging, attempts are being made to discover new contrast agents that have relevant T1 and T2 contrast properties and can be detected by both MRI and optical imaging. Millward et al. (2013) tried to detect inflammatory lesions at the BCSFB at an early stage in a mouse model of multiple sclerosis (95). Intravenous administration of very small superparamagnetic iron oxide particles (VSOPs) was used, as these particles can be phagocytosed (96) and efficiently extravasated, and highlight barrier disruptions (97). VSOPs accumulated in the choroid plexus of mice with T-cell transfer, but without clinical signs of overt inflammation, although this accumulation could only be detected with histology, and not with MRI (95). This accumulation could not be explained by normal diffusion, as it was absent in mice without T-cell transfer. These results indicate that the choroid plexus may be a site for early pathological changes in the immune response of the CNS. At peak disease, VSOP accumulation in the choroid plexus could be detected as hypointense lesions visible on T2*-weighted images 24 hours after intravenous administration, that were not present on pre-contrast images. In a later study, Millward et al. (2019) used europium-doped VSOPs (eu-VSOPs), which have fluorescent properties and can also be detected with fluorescence microscopy $(98,99) .24$ hours after intravenous administration, T2*weighted imaging demonstrated eu-VSOPS accumulation in the choroid plexus in the mouse model of multiple sclerosis. Moreover, the hypointense lesions indicating eu-VSOP accumulation were absent during the remission phase, while mice did show eu-VSOPs enhancement during relapse. 
Table 6.1. Overview of included animal studies

\begin{tabular}{|c|c|c|c|c|c|c|}
\hline Study & Sample & MRI technique & $\begin{array}{l}\text { Contrast agent } \\
\text { type and dosage }\end{array}$ & $\begin{array}{l}\text { Injection } \\
\text { method }\end{array}$ & $\begin{array}{l}\text { Imaging delay time } \\
\text { after contrast } \\
\text { injection }\end{array}$ & Indicator of BCSFB permeability \\
\hline $\begin{array}{l}\text { Nixon et al. } \\
(2008)\end{array}$ & $\begin{array}{l}141 \text { healthy, } \\
\text { female rats }\end{array}$ & $\begin{array}{l}\text { Post-contrast T1- } \\
\text { weighted }\end{array}$ & $\begin{array}{l}100 \mu \mathrm{l} \text { of } 20 \% \\
\text { solution Gd- } \\
\text { DTPA }\end{array}$ & Intravenous & Immediately after & Hyperintense volume in the ventricles \\
\hline $\begin{array}{l}\text { Batra et al. } \\
(2010)\end{array}$ & $\begin{array}{l}\text { Male, } \\
\text { spontaneously } \\
\text { hypertensive rats } \\
6 \text { naive } \\
6 \text { sham surgery } \\
6 \text { MCA0, } 1 \text { hour } \\
\text { since reperfusion } \\
6 \text { MCA0, } 24 \text { hours } \\
\text { since reperfusion } \\
6 \text { MCA0, } 48 \text { hours } \\
\text { since reperfusion }\end{array}$ & $\begin{array}{l}\text { Pre- and post- } \\
\text { contrast FLAIR }\end{array}$ & $0.2 \mathrm{ml}$ Gd-DTPA & Intravenous & 10 minutes & $\begin{array}{l}\text { Gadolinium enhancement ratio of the } \\
\text { ventricles: change in intensity from } \\
\text { the pre-contrast to post-contrast, with } \\
\text { normalization by pre-contrast values }\end{array}$ \\
\hline $\begin{array}{l}\text { Saito et al. } \\
\text { (2011) }\end{array}$ & $\begin{array}{l}20 \text { neonatal, male } \\
\text { rats }\end{array}$ & $\begin{array}{l}\text { Pre- and post- } \\
\text { contrast T1-weighted }\end{array}$ & $\begin{array}{l}0.004 \mathrm{ml} / \mathrm{g} \mathrm{Gd}- \\
\text { DTPA }\end{array}$ & Intravenous & During & $\begin{array}{l}\text { Gadolinium enhancement ratio of the } \\
\text { ventricles: intensity after contrast } \\
\text { divided by intensity before contrast }\end{array}$ \\
\hline $\begin{array}{l}\text { Ichikawa et al. } \\
\text { (2011) }\end{array}$ & $\begin{array}{l}45 \text { male rats with } \\
\text { induced } \\
\text { meningitis }\end{array}$ & $\begin{array}{l}\text { Pre- and post- } \\
\text { contrast T1-weigthed }\end{array}$ & $\begin{array}{l}0.2 \mathrm{mmol} / \mathrm{kg} \mathrm{Gd}- \\
\text { DTPA }\end{array}$ & Intravenous & 5 minutes & $\begin{array}{l}\text { Gadolinium enhancement ratio of the } \\
\text { lateral ventricles: change in intensity } \\
\text { due to contrast compared to the native } \\
\text { signal }\end{array}$ \\
\hline $\begin{array}{l}\text { Yang et al. } \\
(2019)\end{array}$ & $\begin{array}{l}112 \text { mice with } 10 \\
\text { MPDZ knockout }\end{array}$ & $\begin{array}{l}\text { Post-contrast T1- } \\
\text { weighted }\end{array}$ & $\begin{array}{l}0.1 \mathrm{mmol} / \mathrm{kg} \mathrm{Gd}- \\
\text { DTPA }\end{array}$ & Intravenous & Immediately after & $\begin{array}{l}\text { Gadolinium enhancement ratio of the } \\
\text { ventricles: total intensity divided by } \\
\text { the difference between maximum and } \\
\text { minimum intensity }\end{array}$ \\
\hline $\begin{array}{l}\text { Jost et al. } \\
\text { (2016) }\end{array}$ & $\begin{array}{l}18 \text { healthy, male } \\
\text { rats }\end{array}$ & $\begin{array}{l}\text { Pre- and post- } \\
\text { contrast hT2w-FLAIR }\end{array}$ & $\begin{array}{l}5 \text { GBCA groups } 1 \\
\mathrm{mmol} \mathrm{Gd} / \mathrm{kg}\end{array}$ & Intravenous & 1 minute & $\begin{array}{l}\text { Qualitative evaluation of intensity } \\
\text { increase in the CSF spaces }\end{array}$ \\
\hline $\begin{array}{l}\text { Jost et al. } \\
\text { (2017) }\end{array}$ & $\begin{array}{l}48 \text { healthy, male } \\
\text { rats }\end{array}$ & $\begin{array}{l}\text { Pre- and post- } \\
\text { contrast hT2w-FLAIR }\end{array}$ & $\begin{array}{l}7 \text { GBCA groups } \\
1.8 \mathrm{mmol} \mathrm{Gd} / \mathrm{kg}\end{array}$ & Intravenous & $\begin{array}{l}9 \text { and } 24 \text { minutes } \\
\text { and } 4 \text { hours }\end{array}$ & $\begin{array}{l}\text { Gadolinium enhancement ratio of the } \\
\text { CSF cavities: change in intensity from } \\
\text { pre-contrast to post-contrast }\end{array}$ \\
\hline
\end{tabular}


Continuation Table 6.1.

\begin{tabular}{|c|c|c|c|c|c|c|}
\hline Study & Sample & MRI technique & $\begin{array}{l}\text { Contrast agent } \\
\text { type and dosage }\end{array}$ & $\begin{array}{l}\text { Injection } \\
\text { method }\end{array}$ & $\begin{array}{l}\text { Imaging delay time } \\
\text { after contrast } \\
\text { injection }\end{array}$ & Indicator of BCSFB permeability \\
\hline $\begin{array}{l}\text { Nathoo et al. } \\
\text { (2016) }\end{array}$ & $\begin{array}{l}\text { Male rats } \\
2 \text { cold injury } \\
\text { model } \\
5 \text { LPS treated } \\
7 \text { hypoxia }\end{array}$ & $\begin{array}{l}\text { Pre- and post- } \\
\text { contrast T1-weighted }\end{array}$ & $\begin{array}{l}0.5 \mathrm{mmol} / \mathrm{kg} \\
\text { gadodiamide }\end{array}$ & Intravenous & Immediately after & $\begin{array}{l}\text { Average intensity in the ventricles and } \\
\text { periventricular structures of a series } \\
\text { of pre-contrast images and a series of } \\
\text { post-contrast images }\end{array}$ \\
\hline $\begin{array}{l}\text { Aoki et al. } \\
(2004)\end{array}$ & $\begin{array}{l}28 \text { male, healthy } \\
\text { rats }\end{array}$ & $\begin{array}{l}\text { Pre- and post- } \\
\text { contrast T1-weighted }\end{array}$ & $\begin{array}{l}884.3 \mu \mathrm{mol} / \mathrm{kg} \\
\mathrm{MnCl}_{2}\end{array}$ & Intravenous & $\begin{array}{l}0-2 \text { hours, } 1 \text { day, } 2 \\
\text { or } 4 \text { days or } 2 \text { weeks }\end{array}$ & $\begin{array}{l}\text { Change in intensity from the pre- } \\
\text { contrast to post-contrast images in the } \\
\text { choroid plexus, ventricles and CVOs }\end{array}$ \\
\hline $\begin{array}{l}\text { Millward et al. } \\
\text { (2013) }\end{array}$ & $\begin{array}{l}16 \text { female mice } \\
\text { from a MS mouse } \\
\text { model }\end{array}$ & $\begin{array}{l}\text { Pre- and post- } \\
\text { contrast T2*- } \\
\text { weighted }\end{array}$ & $\begin{array}{l}0.2 \mathrm{mmol} / \mathrm{kg} \\
\text { VSOPs }\end{array}$ & Intravenous & 24 hours & $\begin{array}{l}\text { Presence of hypointense lesions in the } \\
\text { choroid plexus compared to pre- } \\
\text { contrast }\end{array}$ \\
\hline $\begin{array}{l}\text { Millward et al. } \\
\text { (2019) }\end{array}$ & $\begin{array}{l}18 \text { female mice } \\
\text { from a MS mouse } \\
\text { model }\end{array}$ & $\begin{array}{l}\text { Pre- and post- } \\
\text { contrast T2*- } \\
\text { weighted }\end{array}$ & $\begin{array}{l}0.2 \mathrm{mmol} / \mathrm{kg} \mathrm{eu}- \\
\text { VSOPs }\end{array}$ & Intravenous & 24 hours & $\begin{array}{l}\text { Presence of hypointense lesions in the } \\
\text { choroid plexus compared to pre- } \\
\text { contrast }\end{array}$ \\
\hline
\end{tabular}

Abbreviations: Gd-DTPA = gadopentetate dimeglumine (gadolinium- diethylenetriamine pentaacetic acid); FLAIR = fluid-attenuated inversion

recovery; MCAO = middle cerebral artery occlusion; MPDZ = gene for the multiple PDZ-domain protein; GBCA = gadolinium-based contrast agent;

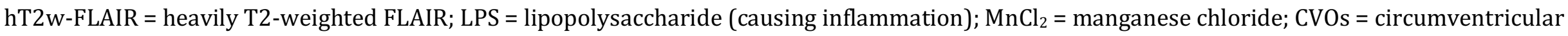

organs; MS = multiple sclerosis; VSOPs = very small superparamagnetic iron oxide particles 
Chapter 6

\subsubsection{Human studies}

See Table 6.2 for a summary of the included human studies.

Hsu et al. (2005) reported on two cases (a child with craniosynosthosis and hydrocephalus and a man with focal seizure) showing diffuse CSF enhancement on postcontrast T1-weighted MRI immediately after intravenous administration of a GBCA compared with the pre-contrast T1-weighted images (100). The authors considered CSF enhancement to be a rare phenomenon that implies BCSFB breakdown, but speculated that, due to developments such as imaging with a longer delay after contrast administration (101) and the use of FLAIR MRI which is more sensitive to low gadolinium concentrations $(102,103)$, CSF enhancement may become more commonly recognized than before.

\subsubsection{Gadolinium enhancement with contrast-enhanced perfusion imaging}

Kao et al. (2003) were the first to demonstrate gadolinium entering the CSF in the lateral ventricles through the choroid plexus after an intravenous GBCA bolus injection in healthy participants (104). Dynamic-susceptibility-contrast (DSC) MR perfusion imaging immediately after intravenous administration of a GBCA was used to measure the spatiotemporal contrast distribution (105). Independent component analysis (106) with thresholding and Bayesian estimation was then used to provide a segmentation of brain tissues. Post-contrast imaging was conducted for 70 seconds, and the results showed gadolinium enhancement in the lateral ventricles delayed by several seconds compared to enhancement in the arteries and white and grey matter, which the authors interpreted as GBCA passage through the BCSFB. Later, Wu et al. (2007) obtained similar results using DSC MR perfusion imaging immediately after intravenous administration of a GBCA in healthy participants and post-contrast imaging conducted for 70 seconds, combined with multivariate Gaussians (107) and the expectation maximization algorithm $(108,109)$.

Bouzerar et al. (2013) investigated choroid plexus functionality using DSC MR perfusion imaging after the first-passage of an intravenous GBCA bolus injection in patients with small cerebral lesions (110). These authors found no relation between age and cerebral blood volume or cerebral blood flow in the choroid plexus (110). However, choroidal capillary permeability significantly decreased with age, which is in accordance with CSF secretion significantly decreasing with age (29). Decrease in capillary 
permeability could be due to a decrease in the exchange area or thickening of the capillary basement membrane (23).

\subsubsection{Gadolinium enhancement with post-contrast T1-weighted and post-contrast} FLAIR imaging

Kim et al. (2020) aimed to investigate changes in choroid plexus permeability in multiple sclerosis (MS) and neuromyelitis optica spectrum disorder (NMOSD) (111), as these are immune-mediated CNS disorders and the choroid plexus is known to regulate the immune response (112). Using T1-weighted imaging immediately after intravenous administration of a GBCA, the ratio between signal intensity in the brightest part of the choroid plexus and the adjacent white matter was calculated. This enhancement ratio was indeed higher for patients with MS and NMSOD relative to healthy controls (111). Due to the absence of a BBB, the choroid plexus always shows enhancement after contrast administration, but the authors state that processes increasing permeability in the choroid plexus capillaries and the BCSFB potentiate contrast extravasation and increase gadolinium enhancement.

\subsubsection{3. $\quad$ Longer delay times}

As contrast agent distribution over the CSF takes time, delayed gadolinium imaging, beyond the usual time of approximately 10 minutes after contrast administration, could be implemented to obtain a better understanding of the CSF pathways (113).

The discovery of the glymphatic system has led to a renewed interest in waste clearance from the brain $(114,115)$. Eide et al. (2019) used pre- and post-contrast T1weighted MRI with a longer delay after intrathecal administration of a GBCA to investigate clearance through the choroid plexus (114). While previous studies focused on $\operatorname{GBCA}$ entering the $\operatorname{CSF}(89,100,104)$, this study focused on GBCA being removed from the CSF. Using pre- and post-contrast T1-weighted MRI, they demonstrated increased signal intensity in the choroid plexus and lateral ventricles at 6-9 hours and 24 hours after intrathecal contrast administration. This was said to indicate that the choroid plexus is involved in clearance from the brain. The enhancement was stronger in idiopathic normal pressure hydrocephalus (iNPH) patients, indicating a slower clearance rate. 
Chapter 6

\subsubsection{New techniques}

So far, studies have primarily been performed in patients with neurologic diseases, for instance in conditions such as acute stroke (116), MS (111), or iNPH (117). More sensitive imaging sequences are needed to investigate subtle changes in BCSFB permeability in healthy individuals.

Deike-Hofman et al. (2019) used hT2w-FLAIR, sensitive to subtle gadolinium enhancement in the CSF, before, and 3 and 24 hours after intravenous administration of a GBCA (118). GBCA distribution through the CNS was visualized and tracked in neurologically healthy patients (Figure 2) and patients with known BBB disruption.

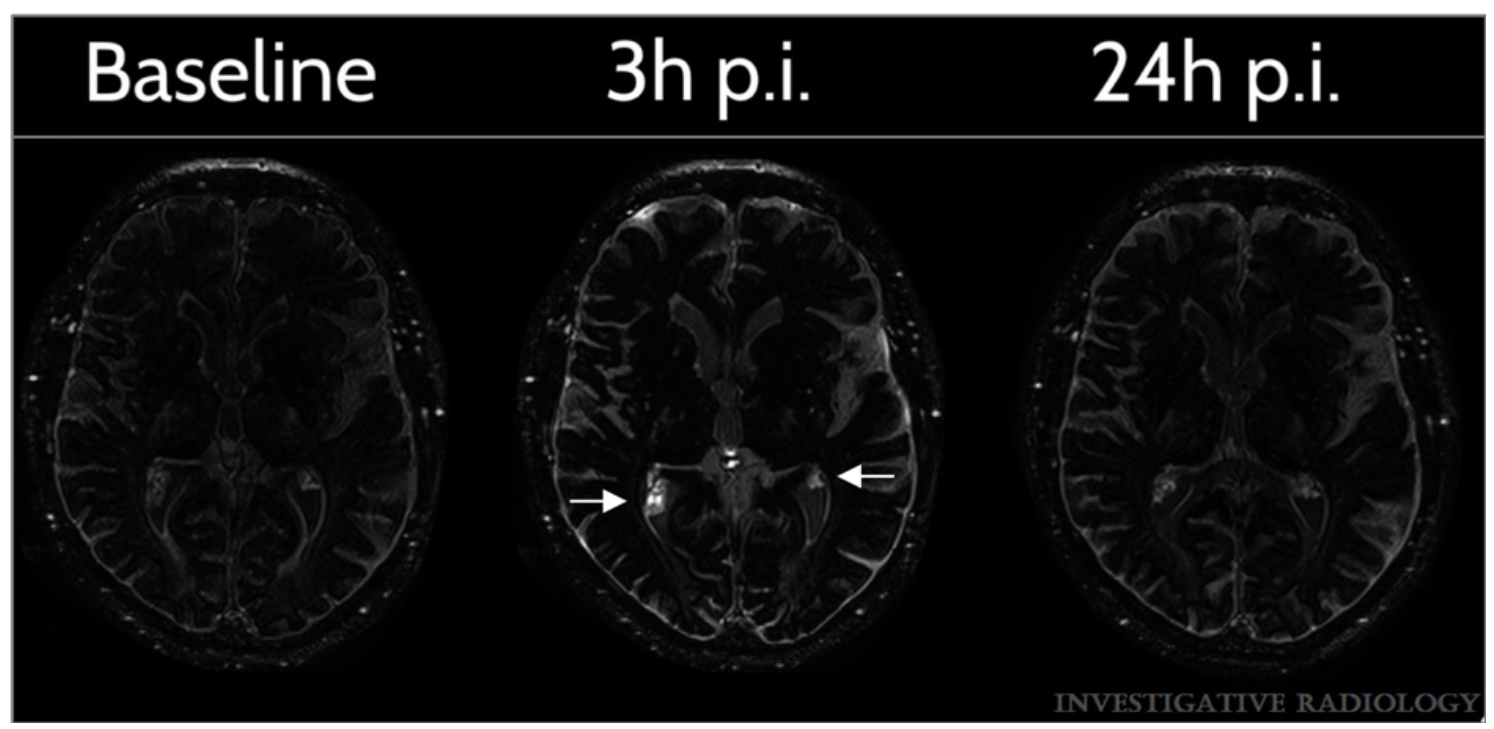

Figure 6.2. Gadolinium enhancement in the choroid plexus using heavily T2-weighted fluidattenuated inversion recovery. Images obtained from a 64-year-old male patient with bronchial carcinoma, without neurological disorder and with normal renal function. Gadolinium enhancement after intravenous administration of a single dose of gadolinium-based contrast agent is detected with heavily T2-weighted fluid-attenuated inversion recovery, with first pre-contrast baseline, then 3 hours post injection (3h p.i.) and lastly 24 hours post injection (24h p.i.). The choroid plexus demonstrates strong contrast enhancement after 3 hours (arrows) compared to baseline. Figure adapted from Deike-Hofman et al. (2019) (118) with permission.

Increase in signal intensity in the post-contrast images compared to the pre-contrast images was calculated. The results demonstrated that the choroid plexus is an important point of entry of GBCAs into the CSF, which implies that the notion of intravenous substances infiltrating the CNS only through BBB disruption needs to be revised. Prolonged presence of GBCAs in the CSF cavities and increased and prolonged 
enhancement of the perivascular spaces $(119,120)$ might be an indicator of glymphatic dysfunction. This GBCA enhancement being associated with the presence of white matter hyperintensities could imply that imaging features of the glymphatic system could have diagnostic value. The study follows the pathway of intact gadolinium complexes through the brain, which might become a diagnostic tool for glymphatic system assessment, and differs from permanent depositions of released gadolinium in the brain (121).

GBCAs can end up in the CSF (120) or brain parenchyma (122), even in healthy individuals. In a recent study, Ohashi et al. (2020) speculated that the pathway by which GBCAs end up in the CNS can be determined by measuring gadolinium enhancement in various CSF spaces (lateral ventricles, Sylvian fissure and cisterns) and in the vitreous, as enhancement is expected to be stronger at locations close to the leakage site (123). The choroid plexus surrounds the lateral ventricles, so if the choroid plexus is the main leakage site, the highest GBCA concentration would be expected in the lateral ventricles. The authors included patients with suspected endolymphatic hydrops, in which excessive endolymph fluid builds up in the inner ear, as this condition was already being evaluated using three-dimensional-real inversion recovery (3D-real IR) imaging. In 3D-real IR imaging, phase-sensitive reconstruction is used instead of magnitude reconstruction. With phase-sensitive reconstruction, signal intensity is also dependent on the polarity of the magnetization, with more negative values displayed darker, allowing visualization of positive and negative magnetization separately (124). Magnitude reconstruction only considers the magnitude of the magnetization, with values around the zero-point being darker. As CSF has a negative magnetization, low gadolinium concentrations can actually bring the value closer to the zero-point and paradoxically decrease signal intensity when using magnitude reconstruction. Phase-sensitive construction prevents this paradoxical signal loss and is better at distinguishing fluid compartments and tissue types $(123,125)$. 3D-real IR was used before, and 5 minutes and 4 hours after intravenous GBCA administration. After 4 hours, significant increase in signal intensity compared to precontrast was found in all CSF locations. However, the signal increase was higher in the cerebral cisternal CSF than in the lateral ventricular CSF. The authors speculate that the choroid plexus could just be one part of the CSF circulation, instead of the main pathway of leakage into the $\operatorname{CSF}(123,126)$, which contradicts previous studies $(89,118)$. 
Chapter 6

\subsubsection{Other contrast agents}

The aforementioned contrast agent manganese has been gaining attention, but in humans, manganese can potentially be neurotoxic (127). Mangafodipir is a chelate that undergoes dephosphorylation and transmetallation after intravenous injection, and slowly releases manganese ions (128). Magnafodipir has been approved and can be used for manganese-enhanced MRI (MEMRI) in humans.

Sudarshana et al. (2019) used MEMRI in healthy participants with T1-weighted MRI before and at various time points after intravenous administration of mangafodipir. (129) The authors demonstrated that structures surrounded by the BBB did not show any manganese enhancement when comparing post-contrast with pre-contrast images, while the choroid plexus did $(129,130)$. Signal intensity in the choroid plexus increased within the first hour after contrast administration, and reached maximum intensity after a time interval of 11 to 40 minutes. Signal intensity was returned to pre-contrast level around 5 to 7 days after administration. However, as opposed to the findings in rats, the CSF and surroundings of the brain parenchyma did not show any manganese enhancement, but the manganese dose derived from mangafodipir was also much lower than the dose derived from manganese chloride delivered to rats. Manganese in the blood can be transported over the BBB as well as the BCSFB, but as the blood levels increase, the manganese ions preferably accumulate for transport into the CSF at the choroid plexus $(131,132)$. Within the choroid plexus, the distribution of manganese is similar to that of gadolinium. Manganese enhancement is, however, thought to especially visualize the cellular components of tissue, and not the intravascular spaces, which may facilitate delineation of tissue borders (Figure 3) (129). 


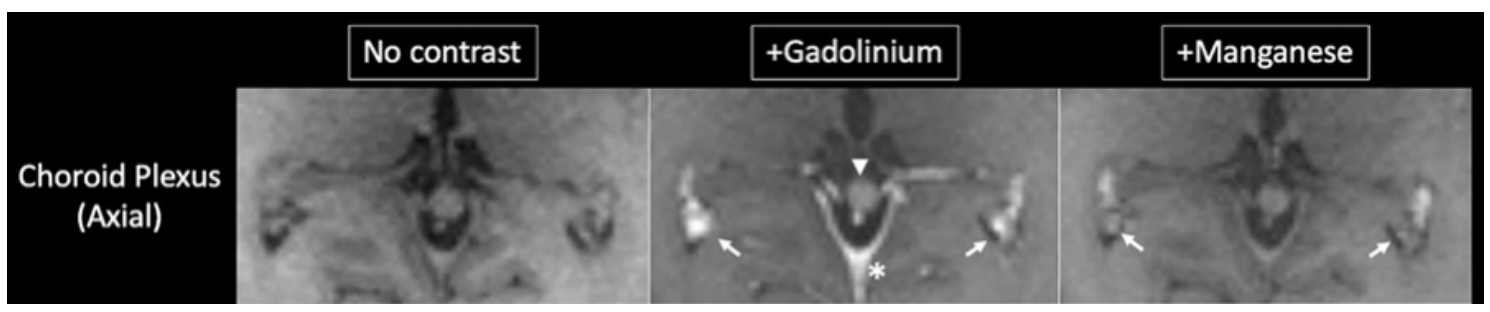

Figure 3. Gadolinium enhancement compared to manganese enhancement. Images obtained approximately 20 minutes after gadolinium administration and 1 hour after mangafodipir administration. After intravenous administration of a gadolinium-based contrast agent, enhancement can be seen in the choroid plexus (arrows), posterior falx cerebri (asterisk), and pineal gland (arrowhead). After intravenous administration of mangafodipir, however, the choroid glomus enhances in a heterogeneous manner. Note the enhancement of the vasculature (posterior falx cerebri (asterisk) where the posterior meningeal artery can be found) with gadolinium, but not with manganese. Figure adapted from Sudarshana et al. (2019) (129) with permission. 
Table 6.2. Overview of included human studies

\begin{tabular}{|c|c|c|c|c|c|c|c|}
\hline Study & Sample & Age in years & $\begin{array}{l}\text { MRI } \\
\text { technique }\end{array}$ & $\begin{array}{l}\text { Contrast agent } \\
\text { type and dosage }\end{array}$ & $\begin{array}{l}\text { Injection } \\
\text { method }\end{array}$ & $\begin{array}{l}\text { Imaging delay } \\
\text { time after } \\
\text { contrast injection }\end{array}$ & $\begin{array}{l}\text { Indicator of BCSFB } \\
\text { permeability }\end{array}$ \\
\hline $\begin{array}{l}\text { Hsu et al. } \\
\text { (2005) }\end{array}$ & $\begin{array}{l}\text { Child with } \\
\text { craniosynosthosis and } \\
\text { hydrocephalus } \\
\text { Man with focal } \\
\text { seizure }\end{array}$ & 5 and 52 & $\begin{array}{l}\text { Pre- and post- } \\
\text { contrast T1- } \\
\text { weighted }\end{array}$ & $\begin{array}{l}0.1 \mathrm{mmol} / \mathrm{kg} \\
\text { magnevist }\end{array}$ & Intravenous & Immediately after & $\begin{array}{l}\text { Presence of diffuse CSF } \\
\text { enhancement compared to } \\
\text { pre-contrast }\end{array}$ \\
\hline $\begin{array}{l}\text { Kao et al. } \\
(2003)\end{array}$ & 5 healthy participants & $\begin{array}{l}\text { Age range: } \\
18-47\end{array}$ & $\begin{array}{l}\text { DSC MR } \\
\text { perfusion }\end{array}$ & $\begin{array}{l}20 \mathrm{ml} 0.5 \\
\mathrm{mmol} / \mathrm{ml} \mathrm{Gd}- \\
\text { DTPA-BMA }\end{array}$ & Intravenous & Immediately after & $\begin{array}{l}\text { Hemodynamic parameter } \\
\text { calculation of the lateral } \\
\text { ventricles }\end{array}$ \\
\hline $\begin{array}{l}\text { Wu et al. } \\
(2007)\end{array}$ & 5 healthy participants & $\begin{array}{l}\text { Age range: } \\
18-47\end{array}$ & $\begin{array}{l}\text { DSC MR } \\
\text { perfusion }\end{array}$ & $\begin{array}{l}20 \mathrm{ml} 0.5 \\
\mathrm{mmol} / \mathrm{ml} \mathrm{Gd}- \\
\text { DTPA-BMA }\end{array}$ & Intravenous & Immediately after & $\begin{array}{l}\text { Hemodynamic parameter } \\
\text { calculation of the lateral } \\
\text { ventricles }\end{array}$ \\
\hline $\begin{array}{l}\text { Bouzerar et al. } \\
(2013)\end{array}$ & $\begin{array}{l}15 \text { patients with small } \\
\text { cerebral lesions }\end{array}$ & $\begin{array}{l}\text { Age range: } \\
21-68\end{array}$ & $\begin{array}{l}\text { DSC MR } \\
\text { perfusion }\end{array}$ & $\begin{array}{l}0.1 \mathrm{ml} / \mathrm{kg} \mathrm{Gd}- \\
\text { DOTA }\end{array}$ & Intravenous & Immediately after & $\begin{array}{l}\text { Hemodynamic parameter } \\
\text { calculation of the choroid } \\
\text { plexus }\end{array}$ \\
\hline $\begin{array}{l}\text { Kim et al. } \\
(2020)\end{array}$ & $\begin{array}{l}51 \text { MS patients } \\
32 \text { NMOSD patients } \\
28 \text { healthy controls }\end{array}$ & $\begin{array}{l}\text { Median age } \\
\text { MS: } 39 \\
\text { Median age } \\
\text { NMSOD: } 50 \\
\text { Median age } \\
\text { healthy } \\
\text { controls: } 48\end{array}$ & $\begin{array}{l}\text { Post-contrast } \\
\text { T1-weighted }\end{array}$ & $\begin{array}{l}\text { Gadolinium- } \\
\text { based }\end{array}$ & Intravenous & Immediately after & $\begin{array}{l}\text { Ratio between intensity in } \\
\text { the brightest part of the } \\
\text { choroid plexus and adjacent } \\
\text { white matter }\end{array}$ \\
\hline $\begin{array}{l}\text { Eide et al. } \\
(2019)\end{array}$ & $\begin{array}{l}8 \text { healthy controls } \\
9 \text { iNPH patients }\end{array}$ & $\begin{array}{l}\text { Mean age } \\
\text { healthy } \\
\text { controls: } 38 \\
\text { Mean age } \\
\text { iNPH } \\
\text { patients: } 68\end{array}$ & $\begin{array}{l}\text { Pre- and post- } \\
\text { contrast T1- } \\
\text { weighted }\end{array}$ & $\begin{array}{l}0.5 \mathrm{ml} 1.0 \\
\mathrm{mmol} / \mathrm{ml} \\
\text { gadobutrol }\end{array}$ & Intrathecal & $\begin{array}{l}1.5-2, \\
2-4 \\
4-6, \\
6-9, \\
24 \text { and } 48 \text { hours }\end{array}$ & $\begin{array}{l}\text { Larger intensity increase in } \\
\text { the choroid plexus and } \\
\text { lateral ventricles after a } \\
\text { longer delay time means } \\
\text { impaired clearance function } \\
\text { at the BCSFB }\end{array}$ \\
\hline
\end{tabular}


Continuation Table 6.2.

\begin{tabular}{|c|c|c|c|c|c|c|c|}
\hline Study & Sample & Age in years & $\begin{array}{l}\text { MRI } \\
\text { technique }\end{array}$ & $\begin{array}{l}\text { Contrast agent } \\
\text { type and dosage }\end{array}$ & $\begin{array}{l}\text { Injection } \\
\text { method }\end{array}$ & $\begin{array}{l}\text { Imaging delay } \\
\text { time after } \\
\text { contrast injection }\end{array}$ & $\begin{array}{l}\text { Indicator of BCSFB } \\
\text { permeability }\end{array}$ \\
\hline $\begin{array}{l}\text { Deike-Hofman } \\
\text { et al. } \\
\text { (2019) }\end{array}$ & $\begin{array}{l}7 \text { patients with } \\
\text { cerebral metastases } \\
33 \text { neurologically } \\
\text { healthy patients }\end{array}$ & $\begin{array}{l}\text { Mean age } \\
\text { cerebral } \\
\text { metastases } \\
\text { patients: } 66 \\
\text { Mean age } \\
\text { neurologically } \\
\text { healthy } \\
\text { patients: } 64\end{array}$ & $\begin{array}{l}\text { Pre- and post- } \\
\text { contrast hT2w- } \\
\text { FLAIR }\end{array}$ & $\begin{array}{l}0.1 \mathrm{ml} / \mathrm{kg} \\
\text { gadovist }\end{array}$ & Intravenous & 3 and 24 hours & $\begin{array}{l}\text { Intensity increase in the } \\
\text { choroid plexus and CSF } \\
\text { cavities }\end{array}$ \\
\hline $\begin{array}{l}\text { Ohashi et al. } \\
(2020)\end{array}$ & $\begin{array}{l}26 \text { patients with } \\
\text { suspected } \\
\text { endolymphatic } \\
\text { hydrops }\end{array}$ & $\begin{array}{l}\text { Age range: } \\
\text { 21-80 years }\end{array}$ & 3D-real-IR & $\begin{array}{l}0.1 \mathrm{mmol} / \mathrm{kg} \mathrm{Gd}- \\
\text { HP-D03A }\end{array}$ & Intravenous & $\begin{array}{l}5 \text { minutes and } 4 \\
\text { hours }\end{array}$ & $\begin{array}{l}\text { Intensity increase in the } \\
\text { lateral ventricle, Sylvian } \\
\text { fissure and cisterns }\end{array}$ \\
\hline $\begin{array}{l}\text { Sudarshana et } \\
\text { al. } \\
(2019)\end{array}$ & 8 healthy participants & $\begin{array}{l}\text { Age range: } \\
18-70 \text { years }\end{array}$ & $\begin{array}{l}\text { Pre- and post- } \\
\text { contrast } \\
\text { MEMRI }\end{array}$ & $\begin{array}{l}5 \mu \mathrm{mol} / \mathrm{kg} \\
\text { Mangafodipir } \\
\text { trisodium }\end{array}$ & Intravenous & $\begin{array}{l}0-2,4 \text { and/or } 6 \\
\text { hours } \\
1,2,3,4,5,6 \\
\text { and/or } 7 \text { days } \\
1,2 \text { and/or } 3 \\
\text { months }\end{array}$ & $\begin{array}{l}\text { Intensity increase in the } \\
\text { choroid plexus }\end{array}$ \\
\hline
\end{tabular}

Abbreviations: DSC = dynamic-susceptibility-contrast; Gd-DTPA-BMA = gadodiamide (gadolinium- diethylenetriamine pentaacetic acid

bismethylamide); Gd-DOTA = gadoterate meglumine (gadolinium- dodecane tetraacetic acid); SWI = susceptibility-weighted imaging; FLAIR = fluidattenuated inversion recovery; Gd-DTPA = gadopentetate dimeglumine (gadolinium- diethylenetriamine pentaacetic acid); MS = multiple sclerosis; NMSOD = neuromyelitis optica spectrum disorder; iNPH = idiopathic normal pressure hydrocephalus; hT2w-FLAIR = heavily T2-weighted FLAIR; 3D-real-IR = three-dimensional-real inversion recovery; Gd-HP-D03A = gadoteridol (gadolinium-1,4,7- tris (carboxymethyl)-10-(2' hydroxypropyl)1,4,7-10-tetraazacyclododecane); MEMRI = manganese-enhanced MRI 
Chapter 6

\subsection{Discussion}

The role of BBB disruption in pathological disorders such as AD and in processes such as aging and neurodegeneration has been gaining great interest over the last decade $(2,133$, 134). As the BBB is important for exchanging vital biomolecules, blocking pathogens and clearing waste products from the brain tissue, BBB disruption can lead to disruption of homeostasis in the CNS and disturb the chemical balance required for neuronal functioning (4). However, the composition of the CSF is also important for the balance in the CNS, and BCSFB function is essential in maintaining this composition (20). The BCSFB has already been demonstrated to deteriorate during aging, and this deterioration is exacerbated in $\operatorname{AD}(10,24)$. Moreover, the choroid plexus epithelial cells of the BCSFB are essential for T-lymphocyte signaling, which is involved in neuroprotection and hippocampus-dependent cognitive functions $(50,51)$. Aging has been associated with dysregulation of T-lymphocyte signaling in the choroid plexus, which can have consequences for CNS integrity and cognitive functioning (54). The role of BCSFB disruption in brain pathology and neurodegeneration should be further investigated, starting with a greater focus on imaging techniques to measure BCSFB permeability.

\subsubsection{Developments in contrast-enhanced MRI for blood-cerebrospinal fluid} barrier permeability assessment

Post-contrast T1-weighted and FLAIR imaging with intravenous administration of a GBCA have been used to detect gadolinium enhancement in the ventricles, and it was concluded that gadolinium can enter the CSF through the BCSFB. Most of these studies, both in animals and humans, were conducted under pathological conditions, such as genetic deficits (83), meningitis (80), acute stroke (116) or MS (111). In these conditions, signal intensity in the ventricles increased after contrast administration, which was thought to be indicative of BCSFB disruption.

The presence of gadolinium in the CSF has been confirmed in a study that used mass spectrometry of CSF samples obtained with lumbar puncture from patients who had previously undergone gadolinium-enhanced MRI (135). CSF samples were obtained at random time points after contrast-enhanced imaging, and gadolinium in the CSF could be detected already 1.1 hours after administration, and could still be detected up to 24 days after administration. Moreover, patients were said to have an intact BBB with a CSF total protein concentration lower than $35 \mathrm{mg} / \mathrm{dl}$, and even in these patients, gadolinium 
could be detected more than 10 days after administration. According to the authors, this immediate and persistent gadolinium enhancement in the CSF, even with an intact BBB, suggests that the choroid plexus, arachnoid granulations and glymphatic system all play a role in CSF and CNS gadolinium distribution.

In vivo imaging studies are also investigating longer imaging delay times after contrast administration. Gadolinium enhancement was demonstrated to peak several hours after contrast administration (136). To visualize the CSF pathways in the brain, imaging should be conducted over an extended time period. Although this will not give additional information on the initial blood-to-CSF transfer, investigating whether contrast accumulates near the choroid plexus after several hours can provide information on the involvement of the BCSFB in clearing compounds from the CSF.

Another promising new development is the use of hT2w-FLAIR imaging, which is more sensitive to subtle gadolinium leakage into the CSF compared to regular FLAIR scans (with shorter echo times), and has already been successfully applied in both rats and humans $(86,118)$. 3D-real IR imaging, which takes into account the negative magnetization of the CSF, is another promising technique (123). Both of these techniques can be used to detect subtle contrast enhancement in the CSF of healthy subjects.

Studies are also considering the use of other contrast agents than gadolinium. Manganese ions, for example, appear hyperintense on post-contrast T1-weighted images and have a longer enhancement window after administration compared to GBCAs (93). Manganese-enhanced (ME) MRI has demonstrated that manganese accumulates in the choroid plexus in both rats and humans $(94,129)$. While in the rat brain, manganese enhancement could also be detected in the CSF and periventricular structures (94), this has not been found in humans yet, where lower concentrations were used (129). Animal studies are also being conducted using VSOPs as contrast agent, as these particles can be used for cell labelling through phagocytosis and highlight barrier disruptions (95). Another recent development is the combination of MRI with optical imaging. In mice studies, eu-VSOPs have been administered, which have superparamagnetic as well as fluorescence properties, and can be used not only for contrast-enhanced MRI, but also for fluorescence microscopy and immunostaining with the advantage of being able to confirm the in vivo findings (98). 
It is important to note that, while this review exclusively focused on contrast-enhanced MRI, there could be contrast-free approaches to assess BCSFB integrity. A recent, important development is for instance the use of non-invasive, tracer-free BCSFB arterial spin labelling (ASL) (137). An ASL MRI sequence is used to label blood water in the feeding arteries and capture how this water crosses the BCSFB into the ventricular CSF. An ultra-long echo time is used, so that the signal from the blood and brain tissue will almost fully decay, while signal from the CSF is preserved due to the long T2 relaxation time of the CSF. Also, low spatial resolution is used, so that small signals can be detected (i.e., discerned from noise) due to the relatively large voxel size, as partial volume effects from the surrounding brain tissue cannot influence the signal due to the ultra-long echo time. Applying this technique, a significantly decreased BCSFB-mediated water delivery in aged mice relative to adult mice was found, and the authors state that these findings suggest that BCSFB function might play an important role in the initial stages of neurodegeneration and might meet the urgent need for early biomarkers of neurodegeneration. BCSFB ASL still needs to be investigated in the human brain, and it would be interesting if future studies could compare this promising method to the contrast-enhanced MRI methods described in this review.

\subsubsection{Limitations}

While the present review provides a comprehensive overview of the available studies that assessed BCSFB permeability with contrast-enhanced MRI, there are several limitations that complicate the interpretation of the reported findings. Firstly, the presence of contrast material in the CSF does not necessarily have to imply BCSFB breakdown, as there are alternative routes through which contrast agents can reach the CSF (136). For example, BBB breakdown could be another route, although contrast agents passing from the blood into the brain parenchyma and then being cleared into the CSF is generally thought to take longer (138). Other points through which contrast agents can enter the CSF, even in healthy conditions, are the anterior eye segment (139), the peripheral part of the cranial nerves (113) and the CVOs (140). Also, several recent studies have demonstrated that intravenously administered contrast agent can leak from the cortical veins into the surrounding CSF (141-143). The contrast agent can permeate the sparse pial sheath of the venous wall of the cortical veins into the subarachnoid space. With longer delay after contrast administration, the contrast agent can also be distributed 
over the CSF by the glymphatic system (144). Recently, the existence of meningeal lymphatic vessels along the superior sagittal sinus was discovered $(145,146)$. These meningeal lymphatic vessels are thought to drain CSF into the cervical lymph nodes, and are for instance involved in the clearance of $A \beta(147,148)$. CSF enhancement could thus be attributed to a mix of BBB disruption, BCSFB disruption and glymphatic dysfunction (136).

Secondly, CSF contrast enhancement is not only related to contrast agent entering the CSF, but probably also to the CSF secretion rate and turnover. Especially at longer delay times after contrast administration, any leaked contrast will remain in the CSF longer when the CSF clearance rate is lower (149).

Thirdly, the extent of CSF enhancement due to BCSFB disruption is probably dependent on the contrast agent dosage, the specific MR sequence parameters used, and the assessment method of CSF enhancement (i.e., visual inspection, contrast enhancement ratio, hemodynamic parameter calculation, etc.). For this reason, it is very difficult to directly compare the findings of the individual studies in this review.

Finally, the detection of contrast extravasation at the level of the choroid plexus does not necessarily have to indicate BCSFB disruption, as it is part of normal choroid plexus and BCSFB functioning to secrete filtrated blood plasma into the CSF (10). Also, several studies conducted in healthy animals and humans have demonstrated postcontrast changes at the level of the choroid plexus and ventricles $(89,118,123)$. When using contrast-enhanced MRI to assess BCSFB functioning, it remains unclear to what extent the findings can be attributed to normal functioning, or BCSFB disruption. Future studies should therefore include appropriate control samples for comparison.

\subsection{Conclusion}

The BCSFB is involved in the composition of the CSF and thus the maintenance of homeostasis in the CNS, but how BCSFB disruption relates to age-related neurodegeneration is underassessed. This review focused on how contrast enhancement on MRI has been used to assess BCSFB permeability. Post-contrast gadolinium enhancement in the ventricles has revealed BCSFB disruption and new techniques are being developed, for instance imaging sequences that are highly sensitive to the CSF (hT2w-FLAIR, 3D-real IR) or the use of contrast agents other than gadolinium-based 


\section{Chapter 6}

compounds (manganese, VSOPs). By further promoting these sorts of developments, we believe much progress can still be made in the assessment of BCSFB integrity.

\subsection{Declaration of interest}

The authors declare that they have no competing interests.

\subsection{References}

1. Erdő F, Denes L, de Lange E. Age-associated physiological and pathological changes at the bloodbrain barrier: a review. Journal of Cerebral Blood Flow \& Metabolism. 2017;37(1):4-24.

2. van de Haar HJ, Burgmans S, Jansen JF, van Osch MJ, van Buchem MA, Muller M, et al. Blood-brain barrier leakage in patients with early Alzheimer disease. Radiology. 2016;281(2):527-35.

3. Montagne A, Zhao Z, Zlokovic BV. Alzheimer's disease: A matter of blood-brain barrier dysfunction? Journal of Experimental Medicine. 2017;214(11):3151-69.

4. Zlokovic BV. The blood-brain barrier in health and chronic neurodegenerative disorders. Neuron. 2008;57(2):178-201.

5. Johanson CE. Chapter 11 - Choroid Plexus-Cerebrospinal Fluid Transport Dynamics: Support of Brain Health and a Role in Neurotherapeutics. In: Conn PM, editor. Conn's Translational Neuroscience. San Diego: Academic Press; 2017. p. 233-61.

6. Ghersi-Egea JF, Strazielle N. Brain drug delivery, drug metabolism, and multidrug resistance at the choroid plexus. Microscopy research and technique. 2001;52(1):83-8.

7. Starr JM, Farrall AJ, Armitage P, McGurn B, Wardlaw J. Blood-brain barrier permeability in Alzheimer's disease: a case-control MRI study. Psychiatry Research: Neuroimaging. 2009;171(3):232-41.

8. Liddelow SA. Development of the choroid plexus and blood-CSF barrier. Frontiers in Neuroscience. $2015 ; 9(32)$.

9. Wolburg H, Paulus W. Choroid plexus: biology and pathology. Acta neuropathologica. 2010;119(1):75-88.

10. Balusu S, Brkic M, Libert C, Vandenbroucke RE. The choroid plexus-cerebrospinal fluid interface in Alzheimer's disease: more than just a barrier. Neural regeneration research. 2016;11(4):534.

11. Khasawneh AH, Garling RJ, Harris CA. Cerebrospinal fluid circulation: What do we know and how do we know it? Brain circulation. 2018;4(1):14.

12. Brinker T, Stopa E, Morrison J, Klinge P. A new look at cerebrospinal fluid circulation. Fluids Barriers CNS. 2014;11:10.

13. Orešković D, Klarica M. The formation of cerebrospinal fluid: Nearly a hundred years of interpretations and misinterpretations. Brain Research Reviews. 2010;64(2):241-62.

14. Orešković D, Klarica M. A new look at cerebrospinal fluid movement. Fluids and Barriers of the CNS. 2014;11(1):16.

15. Spector R, Robert Snodgrass S, Johanson CE. A balanced view of the cerebrospinal fluid composition and functions: Focus on adult humans. Experimental Neurology. 2015;273:57-68. 
16. Bateman GA, Brown KM. The measurement of CSF flow through the aqueduct in normal and hydrocephalic children: from where does it come, to where does it go? Child's Nervous System. 2012;28(1):55-63.

17. Hladky SB, Barrand MA. Mechanisms of fluid movement into, through and out of the brain: evaluation of the evidence. Fluids and Barriers of the CNS. 2014;11(1):1.

18. Spector R, Keep RF, Robert Snodgrass S, Smith QR, Johanson CE. A balanced view of choroid plexus structure and function: Focus on adult humans. Experimental Neurology. 2015;267:78-86.

19. Davson H, Segal MB. Physiology of the CSF and blood-brain barriers: CRC press; 1996.

20. Segal MB. The choroid plexuses and the barriers between the blood and the cerebrospinal fluid. Cellular and molecular neurobiology. 2000;20(2):183-96.

21. Serot J, Christmann D, Dubost T, Couturier M. Cerebrospinal fluid transthyretin: aging and late onset Alzheimer's disease. Journal of Neurology, Neurosurgery \& Psychiatry. 1997;63(4):506-8.

22. Borlongan C, Skinner S, Geaney M, Vasconcellos A, Elliott R, Emerich D. CNS grafts of rat choroid plexus protect against cerebral ischemia in adult rats. Neuroreport. 2004;15(10):1543-7.

23. Serot JM, Foliguet B, Béné MC, Faure GC. Choroid plexus and ageing in rats: a morphometric and ultrastructural study. European Journal of Neuroscience. 2001;14(5):794-8.

24. Serot J-M, Béné M-C, Faure GC. Choroid plexus, aging of the brain, and Alzheimer's disease. Front Biosci. 2003;8(suppl):s515-21.

25. Wen GY, Wisniewski HM, Kascsak RJ. Biondi ring tangles in the choroid plexus of Alzheimer's disease and normal aging brains: a quantitative study. Brain research. 1999;832(1-2):40-6.

26. Preston J, editor Age-related reduction in rat choroid plexus chloride efflux and CSF secretion rate. Soc Neurosci; 1999.

27. Wilson M, Preston J, Thomas S, Segal M. Altered cerebrospinal fluid secretion rate and 125Ilabelled-amyloid transport in the aged sheep isolated perfused choroid plexus. J Physiol(London) P. 1999;515:7P.

28. Chen R, Kassem N, Redzic Z, Chen C, Segal M, Preston J. Age-related changes in choroid plexus and blood-cerebrospinal fluid barrier function in the sheep. Experimental gerontology. 2009;44(4):289-96.

29. May C, Kaye J, Atack JR, Schapiro M, Friedland R, Rapoport S. Cerebrospinal fluid production is reduced in healthy aging. Neurology. 1990;40(3 Part 1):500-.

30. Fishman RA. The cerebrospinal fluid production rate is reduced in dementia of the Alzheimer's type. Neurology. 2002;58(12):1866-.

31. Gideon P, Thomsen C, Ståhlberg F, Henriksen O. Cerebrospinal fluid production and dynamics in normal aging: a MRI phase-mapping study. Acta neurologica scandinavica. 1994;89(5):362-6.

32. Tanna NK, Kohn MI, Horwich DN, Jolles PR, Zimmerman RA, Alves WM, et al. Analysis of brain and cerebrospinal fluid volumes with MR imaging: impact on PET data correction for atrophy. Part II. Aging and Alzheimer dementia. Radiology. 1991;178(1):123-30.

33. Serot J-M, Béné M-C, Foliguet B, Faure GC. Morphological alterations of the choroid plexus in lateonset Alzheimer's disease. Acta neuropathologica. 2000;99(2):105-8.

34. Silverberg G, Heit G, Huhn S, Jaffe R, Chang S, Bronte-Stewart H, et al. The cerebrospinal fluid production rate is reduced in dementia of the Alzheimer's type. Neurology. 2001;57(10):1763-6. 


\section{Chapter 6}

35. Silverberg GD, Mayo M, Saul T, Rubenstein E, McGuire D. Alzheimer's disease, normal-pressure hydrocephalus, and senescent changes in CSF circulatory physiology: a hypothesis. The Lancet Neurology. 2003;2(8):506-11.

36. De Leon M, DeSanti S, Zinkowski R, Mehta P, Pratico D, Segal S, et al. MRI and CSF studies in the early diagnosis of Alzheimer's disease. Journal of internal medicine. 2004;256(3):205-23.

37. Vitek MP, Bhattacharya K, Glendening JM, Stopa E, Vlassara H, Bucala R, et al. Advanced glycation end products contribute to amyloidosis in Alzheimer disease. Proceedings of the National Academy of Sciences. 1994;91(11):4766-70.

38. Chen G-f, Xu T-h, Yan Y, Zhou Y-r, Jiang Y, Melcher K, et al. Amyloid beta: structure, biology and structure-based therapeutic development. Acta Pharmacologica Sinica. 2017;38(9):1205.

39. Vargas T, Ugalde C, Spuch C, Antequera D, Morán MJ, Martín MA, et al. A $\beta$ accumulation in choroid plexus is associated with mitochondrial-induced apoptosis. Neurobiology of aging. 2010;31(9):1569-81.

40. Mesquita SD, Ferreira AC, Sousa JC, Santos NC, Correia-Neves M, Sousa N, et al. Modulation of iron metabolism in aging and in Alzheimer's disease: relevance of the choroid plexus. Frontiers in cellular neuroscience. 2012;6:25.

41. Crossgrove JS, Li GJ, Zheng W. The choroid plexus removes $\beta$-amyloid from brain cerebrospinal fluid. Experimental Biology and Medicine. 2005;230(10):771-6.

42. Li MD, Kane JK, Matta SG, Blaner WS, Sharp BM. Nicotine enhances the biosynthesis and secretion of transthyretin from the choroid plexus in rats: Implications for $\beta$-amyloid formation. Journal of Neuroscience. 2000;20(4):1318-23.

43. Tang YP, Haslam SZ, Conrad SE, Sisk CL. Estrogen increases brain expression of the mRNA encoding transthyretin, an amyloid $\beta$ scavenger protein. Journal of Alzheimer's Disease. 2004;6(4):413-20.

44. Chen RL, Athauda SB, Kassem NA, Zhang Y, Segal MB, Preston JE. Decrease of transthyretin synthesis at the blood-cerebrospinal fluid barrier of old sheep. The Journals of Gerontology Series A: Biological Sciences and Medical Sciences. 2005;60(7):852-8.

45. Ikeda T, Furukawa Y, Mashimoto S, Takahashi K, Yamada M. Vitamin B12 levels in serum and cerebrospinal fluid of people with Alzheimer's disease. Acta Psychiatrica Scandinavica. 1990;82(4):327-9. 46. Serot J, Christmann D, Dubost T, Bene M, Faure G. CSF-folate levels are decreased in late-onset AD patients. Journal of neural transmission. 2001;108(1):93-9.

47. Selhub J, Troen A, Rosenberg IH. B vitamins and the aging brain. Nutrition Reviews. 2010;68(suppl_2):S112-S8.

48. Bergen AA, Kaing S, Jacoline B, Gorgels TG, Janssen SF. Gene expression and functional annotation of human choroid plexus epithelium failure in Alzheimer's disease. BMC genomics. 2015;16(1):956.

49. Baruch K, Kertser A, Porat Z, Schwartz M. Cerebral nitric oxide represses choroid plexus NF $\kappa B-$ dependent gateway activity for leukocyte trafficking. The EMBO journal. 2015;34(13):1816-28.

50. Ziv Y, Ron N, Butovsky O, Landa G, Sudai E, Greenberg N, et al. Immune cells contribute to the maintenance of neurogenesis and spatial learning abilities in adulthood. Nature neuroscience. 2006;9(2):268-75. 
51. Moalem G, Leibowitz-Amit R, Yoles E, Mor F, Cohen IR, Schwartz M. Autoimmune T cells protect neurons from secondary degeneration after central nervous system axotomy. Nature medicine. 1999;5(1):49-55.

52. Engelhardt B, Ransohoff RM. The ins and outs of T-lymphocyte trafficking to the CNS: anatomical sites and molecular mechanisms. Trends in immunology. 2005;26(9):485-95.

53. Emerich DF, Skinner SJ, Borlongan CV, Vasconcellos AV, Thanos CG. The choroid plexus in the rise, fall and repair of the brain. Bioessays. 2005;27(3):262-74.

54. Baruch K, Ron-Harel N, Gal H, Deczkowska A, Shifrut E, Ndifon W, et al. CNS-specific immunity at the choroid plexus shifts toward destructive Th2 inflammation in brain aging. Proceedings of the national academy of sciences. 2013;110(6):2264-9.

55. Shearer GM. Th1/Th2 changes in aging. Mech Ageing Dev. 1997;94(1-3):1-5.

56. Stevenson NJ, Addley MR, Ryan EJ, Boyd CR, Carroll HP, Paunovic V, et al. CCL11 blocks IL-4 and GM-CSF signaling in hematopoietic cells and hinders dendritic cell differentiation via suppressor of cytokine signaling expression. Journal of leukocyte biology. 2009;85(2):289-97.

57. Kesslak JP, So V, Choi J, Cotman CW, Gomez-Pinilla F. Learning upregulates brain-derived neurotrophic factor messenger ribonucleic acid: a mechanism to facilitate encoding and circuit maintenance? Behavioral neuroscience. 1998;112(4):1012.

58. Agarwal N, Contarino C, Toro EF. Neurofluids: A holistic approach to their physiology, interactive dynamics and clinical implications for neurological diseases. Veins and Lymphatics. 2019;8(3).

59. DiNuzzo M, Nedergaard M. Brain energetics during the sleep-wake cycle. Current opinion in neurobiology. 2017;47:65-72.

60. Schubert JJ, Veronese M, Marchitelli L, Bodini B, Tonietto M, Stankoff B, et al. Dynamic 11C-PiB PET shows cerebrospinal fluid flow alterations in Alzheimer disease and multiple sclerosis. Journal of Nuclear Medicine. 2019;60(10):1452-60.

61. Sundaram S, Hughes RL, Peterson E, Müller-Oehring EM, Bronte-Stewart HM, Poston KL, et al. Establishing a framework for neuropathological correlates and glymphatic system functioning in Parkinson's disease. Neuroscience \& Biobehavioral Reviews. 2019;103:305-15.

62. Christensen J, Wright DK, Yamakawa GR, Shultz SR, Mychasiuk R. Repetitive mild traumatic brain injury alters glymphatic clearance rates in limbic structures of adolescent female rats. Scientific reports. 2020;10(1):1-9.

63. Gaberel T, Gakuba C, Goulay R, De Lizarrondo SM, Hanouz J-L, Emery E, et al. Impaired Glymphatic Perfusion After Strokes Revealed by Contrast-Enhanced MRI. Stroke. 2014:STROKEAHA. 114.006617.

64. Iliff JJ, Wang M, Liao Y, Plogg BA, Peng W, Gundersen GA, et al. A paravascular pathway facilitates CSF flow through the brain parenchyma and the clearance of interstitial solutes, including amyloid $\beta$. Science translational medicine. 2012;4(147):147ra11-ra11.

65. Kress BT, Iliff JJ, Xia M, Wang M, Wei HS, Zeppenfeld D, et al. Impairment of paravascular clearance pathways in the aging brain. Annals of neurology. 2014;76(6):845-61.

66. Smith AJ, Verkman AS. CrossTalk opposing view: Going against the flow: interstitial solute transport in brain is diffusive and aquaporin-4 independent. The Journal of Physiology. 2019;597(17):4421-4. 


\section{Chapter 6}

67. Abbott NJ, Pizzo ME, Preston JE, Janigro D, Thorne RG. The role of brain barriers in fluid movement in the CNS: is there a 'glymphatic' system? Acta Neuropathologica. 2018;135(3):387-407.

68. Taoka T, Naganawa S. Imaging for central nervous system (CNS) interstitial fluidopathy: disorders with impaired interstitial fluid dynamics. Japanese Journal of Radiology. 2020.

69. Ott BR, Jones RN, Daiello LA, de la Monte SM, Stopa EG, Johanson CE, et al. Blood-Cerebrospinal Fluid Barrier Gradients in Mild Cognitive Impairment and Alzheimer's Disease: Relationship to Inflammatory Cytokines and Chemokines. Frontiers in Aging Neuroscience. 2018;10(245).

70. Chalbot S, Zetterberg H, Blennow K, Fladby T, Andreasen N, Grundke-Iqbal I, et al. Bloodcerebrospinal fluid barrier permeability in Alzheimer's disease. Journal of Alzheimer's Disease. 2011;25(3):505-15.

71. Goldim MP, Danielski LG, Rodrigues JF, Joaquim L, Garbossa L, de Oliveira Junior AN, et al. Oxidative stress in the choroid plexus contributes to blood-cerebrospinal fluid barrier disruption during sepsis development. Microvascular research. 2019;123:19-24.

72. Okamura T, Ishibashi N, Zurakowski D, Jonas RA. Cardiopulmonary bypass increases permeability of the blood-cerebrospinal fluid barrier. The Annals of thoracic surgery. 2010;89(1):187-94.

73. Raja R, Rosenberg GA, Caprihan A. MRI measurements of Blood-Brain Barrier function in dementia: A review of recent studies. Neuropharmacology. 2018;134:259-71.

74. van de Haar HJ, Burgmans S, Hofman PA, Verhey FR, Jansen JF, Backes WH. Blood-brain barrier impairment in dementia: current and future in vivo assessments. Neuroscience \& Biobehavioral Reviews. 2014.

75. Verheggen IC, de Jong JJ, van Boxtel MP, Gronenschild EH, Palm WM, Postma AA, et al. Increase in blood-brain barrier leakage in healthy, older adults. GeroScience. 2020:1-11.

76. Nixon PF, Jordan L, Zimitat C, Rose SE, Zelaya F. Choroid Plexus Dysfunction: The Initial Event in the Pathogenesis of Wernicke's Encephalopathy and Ethanol Intoxication. Alcoholism: Clinical and Experimental Research. 2008;32(8):1513-23.

77. Montaner J, Alvarez-Sabín J, Molina C, Anglés A, Abilleira S, Arenillas J, et al. Matrix metalloproteinase expression after human cardioembolic stroke: temporal profile and relation to neurological impairment. Stroke. 2001;32(8):1759-66.

78. Batra A, Latour LL, Ruetzler CA, Hallenbeck JM, Spatz M, Warach S, et al. Increased plasma and tissue MMP levels are associated with BCSFB and BBB disruption evident on post-contrast FLAIR after experimental stroke. Journal of Cerebral Blood Flow \& Metabolism. 2010;30(6):1188-99.

79. Saito S, Aoki I, Sawada K, Sun X-Z, Chuang K-H, Kershaw J, et al. Quantitative and noninvasive assessment of prenatal X-ray-induced CNS abnormalities using magnetic resonance imaging. Radiation research. 2011;175(1):1-9.

80. Ichikawa H, Itoh K. Blood-arachnoid barrier disruption in experimental rat meningitis detected using gadolinium-enhancement ratio imaging. Brain research. 2011;1390:142-9.

81. Ichikawa H, Ishikawa M, Fukunaga M, Ishikawa K, Ishiyama H. Quantitative evaluation of bloodcerebrospinal fluid barrier permeability in the rat with experimental meningitis using magnetic resonance imaging. Brain Research. 2010;1321:125-32. 
82. Pfister H-W, Fontana A, Täuber MG, Tomasz A, Scheld WM. Mechanisms of Brain Injury in Bacterial Meningitis: Workshop Summary. Clinical Infectious Diseases. 1994;19(3):463-79.

83. Yang J, Simonneau C, Kilker R, Oakley L, Byrne MD, Nichtova Z, et al. Murine MPDZ-linked hydrocephalus is caused by hyperpermeability of the choroid plexus. EMBO molecular medicine. 2019;11(1):e9540.

84. Feldner A, Adam MG, Tetzlaff F, Moll I, Komljenovic D, Sahm F, et al. Loss of Mpdz impairs ependymal cell integrity leading to perinatal-onset hydrocephalus in mice. EMBO molecular medicine. 2017;9(7):890-905.

85. Milner LC, Shirley RL, Kozell LB, Walter NA, Kruse LC, Komiyama NH, et al. Novel MPDZ/MUPP1 transgenic and knockdown models confirm Mpdz's role in ethanol withdrawal and support its role in voluntary ethanol consumption. Addiction biology. 2015;20(1):143-7.

86. Jost G, Lenhard DC, Sieber MA, Lohrke J, Frenzel T, Pietsch H. Signal increase on unenhanced T1weighted images in the rat brain after repeated, extended doses of gadolinium-based contrast agents: comparison of linear and macrocyclic agents. Investigative radiology. 2016;51(2):83.

87. Naganawa S, Kawai H, Sone M, Nakashima T. Increased sensitivity to low concentration gadolinium contrast by optimized heavily T2-weighted 3D-FLAIR to visualize endolymphatic space. Magnetic Resonance in Medical Sciences. 2010;9(2):73-80.

88. Freeze W, Ter Weele D, Palm W, van Hooren R, Hoff E, Jansen J, et al. Optimal detection of subtle gadolinium leakage in CSF with heavily T2-weighted fluid-attenuated inversion recovery imaging. American Journal of Neuroradiology. 2019;40(9):1481-3.

89. Jost G, Frenzel T, Lohrke J, Lenhard DC, Naganawa S, Pietsch H. Penetration and distribution of gadolinium-based contrast agents into the cerebrospinal fluid in healthy rats: a potential pathway of entry into the brain tissue. European radiology. 2017;27(7):2877-85.

90. Roberts TP, Mikulis D. Neuro MR: principles. Journal of Magnetic Resonance Imaging: An Official Journal of the International Society for Magnetic Resonance in Medicine. 2007;26(4):823-37.

91. Nathoo N, Jalal H, Natah SS, Zhang Q, Wu Y, Dunn JF. Hypoxia and Inflammation-Induced Disruptions of the Blood-Brain and Blood-Cerebrospinal Fluid Barriers Assessed Using a Novel T 1-Based MRI Method. Brain Edema XVI: Springer; 2016. p. 23-8.

92. Natah SS, Srinivasan S, Pittman Q, Zhao Z, Dunn JF. Effects of acute hypoxia and hyperthermia on the permeability of the blood-brain barrier in adult rats. Journal of Applied Physiology. 2009;107(4):134856.

93. Wesolowski JR, Kaiser A. Alternatives to GBCA: Are We There Yet? Topics in Magnetic Resonance Imaging. 2016;25(4):171-5.

94. Aoki I, Wu Y-JL, Silva AC, Lynch RM, Koretsky AP. In vivo detection of neuroarchitecture in the rodent brain using manganese-enhanced MRI. Neuroimage. 2004;22(3):1046-59.

95. Millward JM, Schnorr J, Taupitz M, Wagner S, Wuerfel JT, Infante-Duarte C. Iron oxide magnetic nanoparticles highlight early involvement of the choroid plexus in central nervous system inflammation. 2013. 


\section{Chapter 6}

96. Stroh A, Zimmer C, Werner N, Gertz K, Weir K, Kronenberg G, et al. Tracking of systemically administered mononuclear cells in the ischemic brain by high-field magnetic resonance imaging. Neuroimage. 2006;33(3):886-97.

97. Wuerfel J, Tysiak E, Prozorovski T, Smyth M, Mueller S, Schnorr J, et al. Mouse model mimics multiple sclerosis in the clinico-radiological paradox. European Journal of Neuroscience. 2007;26(1):1908.

98. Millward JM, Ariza de Schellenberger A, Berndt D, Hanke-Vela L, Schellenberger E, Waiczies S, et al. Application of Europium-Doped Very Small Iron Oxide Nanoparticles to Visualize Neuroinflammation with MRI and Fluorescence Microscopy. Neuroscience. 2019;403:136-44.

99. Kobayashi Y, Hauptmann R, Kratz H, Ebert M, Wagner S, Taupitz M. Europium doping of superparamagnetic iron oxide nanoparticles enables their detection by fluorescence microscopy and for quantitative analytics. Technology and Health Care. 2017;25(3):457-70.

100. Hsu HL, Chen CJ. Extensive cerebrospinal fluid enhancement following gadolinium chelate administration: possible pathogenesis. Acta Radiol. 2005;46(5):523-7.

101. KNUTZON RK, POIRIER VC, Gerscovich EO, BROCK JM, BUONOCORE M. The effect of intravenous gadolinium on the magnetic resonance appearance of cerebrospinal fluid. Investigative radiology. 1991;26(7):671-3.

102. Bozzao A, Floris R, Fasoli F, Fantozzi L, Colonnese C, Simonetti G. Cerebrospinal fluid changes after intravenous injection of gadolinium chelate: assessment by FLAIR MR imaging. European radiology. 2003;13(3):592-7.

103. Dechambre S, Duprez T, Grandin C, Lecouvet F, Peeters A, Cosnard G. High signal in cerebrospinal fluid mimicking subarachnoid haemorrhage on FLAIR following acute stroke and intravenous contrast medium. Neuroradiology. 2000;42(8):608-11.

104. Kao YH, Guo WY, Wu YT, Liu KC, Chai WY, Lin CY, et al. Hemodynamic segmentation of MR brain perfusion images using independent component analysis, thresholding, and Bayesian estimation. Magn Reson Med. 2003;49(5):885-94.

105. Rempp KA, Brix G, Wenz F, Becker CR, Gückel F, Lorenz WJ. Quantification of regional cerebral blood flow and volume with dynamic susceptibility contrast-enhanced MR imaging. Radiology. 1994;193(3):637-41.

106. Hyvärinen A, Oja E. Independent component analysis: algorithms and applications. Neural networks. 2000;13(4-5):411-30.

107. Bishop CM. Neural networks for pattern recognition: Oxford university press; 1995.

108. Dempster AP, Laird NM, Rubin DB. Maximum likelihood from incomplete data via the EM algorithm. Journal of the Royal Statistical Society: Series B (Methodological). 1977;39(1):1-22.

109. Wu YT, Chou YC, Guo WY, Yeh TC, Hsieh JC. Classification of spatiotemporal hemodynamics from brain perfusion MR images using expectation-maximization estimation with finite mixture of multivariate gaussian distributions. Magn Reson Med. 2007;57(1):181-91.

110. Bouzerar R, Chaarani B, Gondry-Jouet C, Zmudka J, Balédent O. Measurement of choroid plexus perfusion using dynamic susceptibility MR imaging: capillary permeability and age-related changes. Neuroradiology. 2013;55(12):1447-54. 
111. Kim H, Lim Y-M, Kim G, Lee E-J, Lee JH, Kim HW, et al. Choroid plexus changes on magnetic resonance imaging in multiple sclerosis and neuromyelitis optica spectrum disorder. Journal of the Neurological Sciences. 2020:116904.

112. Baecher-Allan C, Kaskow BJ, Weiner HL. Multiple sclerosis: mechanisms and immunotherapy. Neuron. 2018;97(4):742-68.

113. Naganawa S, Suzuki K, Yamazaki M, Sakurai Y. Serial scans in healthy volunteers following intravenous administration of gadoteridol: time course of contrast enhancement in various cranial fluid spaces. Magnetic Resonance in Medical Sciences. 2014;13(1):7-13.

114. Eide PK, Valnes LM, Pripp AH, Mardal K-A, Ringstad G. Delayed clearance of cerebrospinal fluid tracer from choroid plexus in idiopathic normal pressure hydrocephalus. Journal of Cerebral Blood Flow \& Metabolism. 2019:0271678X19874790.

115. Verheggen I, Van Boxtel M, Verhey F, Jansen J, Backes W. Interaction between blood-brain barrier and glymphatic system in solute clearance. Neuroscience \& Biobehavioral Reviews. 2018;90:26-33.

116. Renú A, Laredo C, Lopez-Rueda A, Llull L, Tudela R, San-Roman L, et al. Vessel Wall enhancement and blood-cerebrospinal fluid barrier disruption after mechanical thrombectomy in acute ischemic stroke. Stroke. 2017;48(3):651-7.

117. Eide PK, Ringstad G. Delayed clearance of cerebrospinal fluid tracer from entorhinal cortex in idiopathic normal pressure hydrocephalus: a glymphatic magnetic resonance imaging study. Journal of Cerebral Blood Flow \& Metabolism. 2019;39(7):1355-68.

118. Deike-Hofmann K, Reuter J, Haase R, Paech D, Gnirs R, Bickelhaupt S, et al. Glymphatic pathway of gadolinium-based contrast agents through the brain: overlooked and misinterpreted. Investigative radiology. 2019;54(4):229-37.

119. Freeze W, Schnerr R, Palm W, Jansen J, Jacobs H, Hoff E, et al. Pericortical enhancement on delayed postgadolinium fluid-attenuated inversion recovery images in normal aging, mild cognitive impairment, and Alzheimer disease. American Journal of Neuroradiology. 2017;38(9):1742-7.

120. Naganawa S, Nakane T, Kawai H, Taoka T. Gd-based contrast enhancement of the perivascular spaces in the basal ganglia. Magnetic Resonance in Medical Sciences. 2017;16(1):61-5.

121. Rasschaert M, Weller RO, Schroeder JA, Brochhausen C, Idée JM. Retention of Gadolinium in Brain Parenchyma: Pathways for Speciation, Access, and Distribution. A Critical Review. Journal of Magnetic Resonance Imaging. 2020.

122. Kanda T, Ishii K, Kawaguchi H, Kitajima K, Takenaka D. High signal intensity in the dentate nucleus and globus pallidus on unenhanced T1-weighted MR images: relationship with increasing cumulative dose of a gadolinium-based contrast material. Radiology. 2014;270(3):834-41.

123. Ohashi T, Naganawa S, Iwata S, Kuno K. Distribution of Gadolinium-based Contrast Agent after Leaking into the Cerebrospinal Fluid: Comparison between the Cerebral Cisterns and the Lateral Ventricles. Magnetic Resonance in Medical Sciences. 2020:mp. 2020-0016.

124. Park H, Cho M, Cho Z. Real-value representation in inversion-recovery NMR imaging by use of a phase-correction method. Magnetic resonance in medicine. 1986;3(1):15-23. 


\section{Chapter 6}

125. Naganawa S, Kawai H, Taoka T, Sone M. Improved 3D-real inversion recovery: a robust imaging technique for endolymphatic hydrops after intravenous administration of gadolinium. Magnetic Resonance in Medical Sciences. 2019;18(1):105.

126. Orešković D, Radoš M, Klarica M. Role of choroid plexus in cerebrospinal fluid hydrodynamics. Neuroscience. 2017;354:69-87.

127. Tuschl K, Mills PB, Clayton PT. Manganese and the brain. International review of neurobiology. 110: Elsevier; 2013. p. 277-312.

128. Runge VM. Safety of approved MR contrast media for intravenous injection. Journal of Magnetic Resonance Imaging: An Official Journal of the International Society for Magnetic Resonance in Medicine. 2000;12(2):205-13.

129. Sudarshana DM, Nair G, Dwyer JT, Dewey B, Steele SU, Suto DJ, et al. Manganese-enhanced MRI of the brain in healthy volunteers. American Journal of Neuroradiology. 2019;40(8):1309-16.

130. Wang C, Gordon P, Hustvedt S, Grant D, Sterud AT, Martinsen I, et al. MR imaging properties and pharmacokinetics of MnDPDP in healthy volunteers. Acta Radiologica. 1997;38(5):665-76.

131. Yokel RA. Manganese flux across the blood-brain barrier. Neuromolecular medicine. 2009;11(4):297-310.

132. Schmitt C, Strazielle N, Richaud P, Bouron A, Ghersi-Egea JF. Active transport at the blood-CSF barrier contributes to manganese influx into the brain. Journal of neurochemistry. 2011;117(4):747-56.

133. Farrall AJ, Wardlaw JM. Blood-brain barrier: ageing and microvascular disease-systematic review and meta-analysis. Neurobiology of aging. 2009;30(3):337-52.

134. Freeze WM, Jacobs HI, de Jong JJ, Verheggen IC, Gronenschild EH, Palm WM, et al. White matter hyperintensities mediate the association between blood-brain barrier leakage and information processing speed. Neurobiology of aging. 2020;85:113-22.

135. Nehra AK, McDonald RJ, Bluhm AM, Gunderson TM, Murray DL, Jannetto PJ, et al. Accumulation of gadolinium in human cerebrospinal fluid after gadobutrol-enhanced MR imaging: a prospective observational cohort study. Radiology. 2018;288(2):416-23.

136. Naganawa S, Ito R, Kato Y, Kawai H, Taoka T, Yoshida T, et al. Intracranial Distribution of Intravenously Administered Gadolinium-based Contrast Agent over a Period of 24 Hours: Evaluation with 3D-real IR Imaging and MR Fingerprinting. Magnetic Resonance in Medical Sciences. 2020:mp. 2020-0030. 137. Evans P, Sokolska M, Alves A, Harrison I, Ohene Y, Nahavandi P, et al. Non-Invasive MRI of BloodCerebrospinal Fluid Barrier Function. Nature communications. 2020;11(1):1-11.

138. Gaohua L, Neuhoff S, Johnson TN, Rostami-Hodjegan A, Jamei M. Development of a permeabilitylimited model of the human brain and cerebrospinal fluid (CSF) to integrate known physiological and biological knowledge: Estimating time varying CSF drug concentrations and their variability using in vitro data. Drug metabolism and pharmacokinetics. 2016;31(3):224-33.

139. Naganawa S, Yamazaki M, Kawai H, Sone M, Nakashima T. Contrast enhancement of the anterior eye segment and subarachnoid space: detection in the normal state by heavily T2-weighted 3D FLAIR. Magnetic Resonance in Medical Sciences. 2011;10(3):193-9. 
140. Verheggen IC, de Jong JJ, van Boxtel MP, Postma AA, Verhey FR, Jansen JF, et al. Permeability of the windows of the brain: feasibility of dynamic contrast-enhanced MRI of the circumventricular organs. Fluids and Barriers of the CNS. 2020;17(1):1-13.

141. Naganawa S, Nakane T, Kawai H, Taoka T. Age dependence of gadolinium leakage from the cortical veins into the cerebrospinal fluid assessed with whole brain 3D-real inversion recovery MR imaging. Magnetic Resonance in Medical Sciences. 2019;18(2):163-9.

142. Naganawa S, Ito R, Nakamichi R, Kawamura M, Kawai H, Taoka T, et al. Relationship between parasagittal perivenous cysts and leakage of gadolinium-based contrast agents into the subarachnoid space around the cortical veins after intravenous administration. Magnetic Resonance in Medical Sciences. 2020:mp. 2020-0062.

143. Naganawa S, Nakane T, Kawai H, Taoka T, Kawaguchi H, Maruyama K, et al. Detection of IVgadolinium leakage from the cortical veins into the CSF using MR fingerprinting. Magnetic Resonance in Medical Sciences. 2020;19(2):141.

144. Iliff JJ, Lee H, Yu M, Feng T, Logan J, Nedergaard M, et al. Brain-wide pathway for waste clearance captured by contrast-enhanced MRI. The Journal of clinical investigation. 2013;123(3):1299-309.

145. Aspelund A, Antila S, Proulx ST, Karlsen TV, Karaman S, Detmar M, et al. A dural lymphatic vascular system that drains brain interstitial fluid and macromolecules. Journal of Experimental Medicine. 2015;212(7):991-9.

146. Wu C-H, Lirng J-F, Ling Y-H, Wang Y-F, Wu H-M, Fuh J-L, et al. Noninvasive Characterization of Human Glymphatics and Meningeal Lymphatics in an in vivo Model of Blood-Brain Barrier Leakage. Annals of Neurology.n/a(n/a).

147. Da Mesquita S, Fu Z, Kipnis J. The meningeal lymphatic system: a new player in neurophysiology. Neuron. 2018;100(2):375-88.

148. Naganawa S, Taoka T. The glymphatic system: a review of the challenges in visualizing its structure and function with MR imaging. Magnetic Resonance in Medical Sciences. 2020:rev. 2020-0122.

149. Pul R, Yildiz Ö, Morbiducci F, Skripuletz T, Schwenkenbecher P, Stangel M, et al. CSF levels of angiopoietin-2 do not differ between patients with CSF fluid leakage syndrome and controls. Disease markers. 2015;2015. 



\section{Chapter 7}

\section{Discussion}

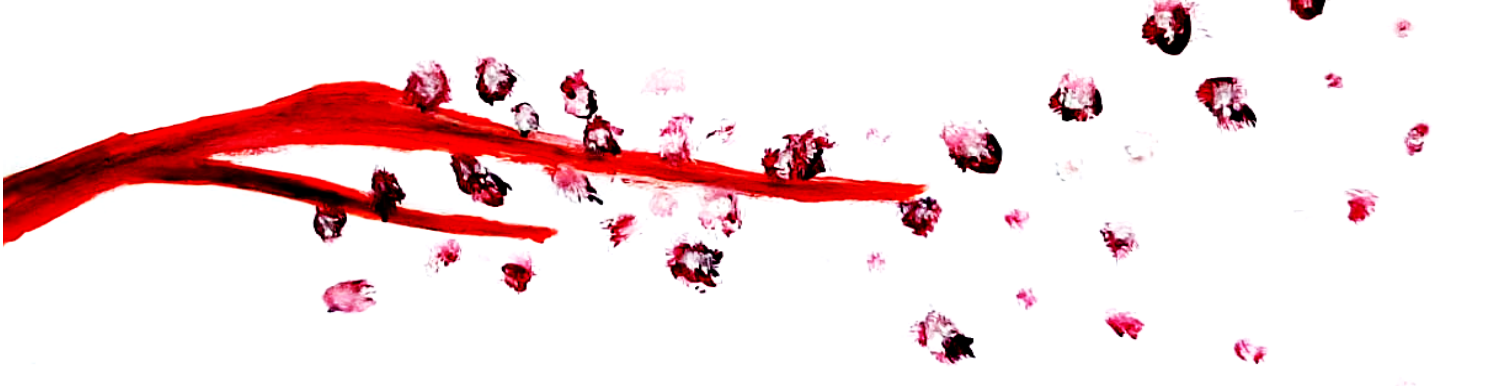




\subsection{Rationale}

Societies are faced with rapidly aging populations (1). Determining what processes are involved in age-related decline has become more important to eventually increase one's chances of successful aging. Neurovascular health seems to influence whether someone ages successfully (2). Neurovascular health depends, among others, on the integrity of the blood-brain barrier (BBB), which is notably affected by age-related changes (3-5). The BBB protects the chemical balance in the interstitial space by regulating the passage of nutrients and pathogens and clearing waste products, which is essential for synaptic and neuronal functioning (6).

Interest in BBB disruption has increased since it became more often associated with pathological conditions, such as Alzheimer's disease (AD) (7-10). BBB disruption can impair the clearance of the amyloid- $\beta$ protein $(A \beta)$, while the neurotoxic effects of $A \beta$ can then again impair BBB integrity (11). With its involvement in both vascular and Alzheimer pathology, BBB disruption might be a common trigger for age-related brain pathology (12). Such changes in brain structure and function are often associated with changes in cognition $(13,14)$, and BBB disruption has indeed been associated with decline in cognitive functioning $(10,15)$. The process of BBB breakdown could be an underlying mechanism of the decline that accompanies aging, even before overt agerelated brain pathology emerges. Recently, interest in BBB disruption has increased due to the development of new imaging techniques capable of measuring even subtle BBB leakage, as can for instance be found in case of dementia (16), with an increasingly used method being dynamic contrast-enhanced magnetic resonance imaging (DCE MRI) (17).

Despite the urgency of the topic and the technical developments, however, DCE MRI studies looking into BBB disruption and its relation to normal aging and age-related cognitive decline had not been conducted before now. We aimed to investigate whether BBB leakage was significantly associated with older age and decline in cognitive performance in a healthy sample. For this purpose, we conducted a study with participants selected from the Maastricht Aging Study (MAAS) (18), in which people were cognitively followed from 1992 until 2005. Middle-aged to older, normal aging individuals, considered healthy, were asked to participate. 'Healthy' was defined as no substantial decrease in global cognition, no cognitive impairment due to substance abuse and no great difficulties in performing activities of daily living, as well as no major neurological or psychiatric condition. We conducted neuropsychological assessment, 
from which we could calculate decline in cognitive performance (encoding, retrieval, processing speed, and inhibition) over a 12-year period using the last measurement of MAAS as baseline, and we performed dual-time resolution DCE MRI which was sensitive enough to detect even the subtle leakage values (white matter, grey matter, hippocampus) found in normal aging individuals.

\subsection{What we learned}

Our results demonstrated that, also in healthy individuals, BBB disruption was associated with age (Chapter 2). We found a higher BBB leakage rate in the total cerebral white and grey matter for older individuals. We also categorized certain brain regions. Primary regions are involved in sensory and motor functions and develop first and deteriorate last. Secondary regions are the association areas that develop later and deteriorate sooner relative to the primary regions. Tertiary regions are involved in higher order cognitive functions and develop last and deteriorate first $(19,20)$. The association between BBB leakage and age was significant in the tertiary regions, but not in the primary or secondary regions. These results show that BBB disruption increases with age especially in those regions that are known to be most vulnerable to normal age-related deterioration. As detrimental age-related processes are known to especially affect the tertiary regions $(21,22)$, this provides evidence that BBB breakdown is one of these processes and supports the notion that it could underlie normal age-related deterioration.

When investigating the relation between BBB disruption and normal age-related cognitive decline, we found a significant association between BBB leakage and 12-year decline in delayed recall score, which can be considered a measure of memory retrieval (Chapter 3). For the retrieval function, both hippocampal and cortical processing is required with activation of a hippocampal-cortical network $(23,24)$. Especially pathology in the white matter tracts can disrupt communication between the hippocampus and cortex and may impair network function. This corresponds to decline in retrieval being strongly related to BBB disruption throughout the cerebral white matter, rather than specifically in the hippocampus. Normal aging can lead to a disruption in communication between the medial temporal and frontal system (25). This could explain why retrieval could be most vulnerable to normal age-related decline and was the only cognitive 
domain significantly associated with BBB disruption, as a possible underlying factor of normal age-related decline.

As a way of further validating the technique, we used dual-time resolution DCE MRI to measure contrast transfer in the circumventricular organs (CVOs) (Chapter 4), known to lack a BBB (26). We indeed found significantly positive transfer constants in the CVOs, higher than in the normal-appearing brain matter and highest for the secretory CVOs. This introduces DCE MRI as a possible method to assess CVO permeability and how pathogens can potentially enter the brain via these structures (27).

Following these empirical studies, we conducted two review studies. In these studies, we broadened our scope from BBB function to other mechanisms involved in maintaining homeostasis. Waste products were previously thought to be primarily cleared from the brain by BBB transport (28), but then the glymphatic system was discovered as waste clearance mechanism. The glymphatic system is a waste clearance system using the microcirculation of the cerebrospinal fluid (CSF), in which the CSF is exchanged with interstitial fluid (ISF) to clear solutes from the interstitial space $(29,30)$. Therefore, we conducted a review focussing on waste clearance by BBB transport and glymphatic function, and how these two clearance systems interact (Chapter 5). BBB transport and glymphatic clearance likely serve complementary roles with partially overlapping mechanisms to provide a well-balanced neuronal environment (30). Properly functioning waste clearance systems protect against the accumulation of toxic waste products such as $A \beta$, and may delay or even prevent the onset of neurodegenerative disorders such as AD. Keeping these clearance systems in good condition could be a new focus for future health initiatives, and for instance emphasize the importance of sleep for brain health (31-33).

Finally, we were interested in the blood-CSF barrier (BCSFB), formed by the choroid plexus epithelial cells $(34,35)$. The BCSFB is involved in the composition of the CSF and thus the maintenance of homeostasis in the central nervous system (CNS) (34). While the relation between BBB leakage, aging and neurodegeneration is being extensively studied using contrast-enhanced MRI (17), the use of this type of studies to investigate how BCSFB leakage relates to age-related neurodegeneration has received little attention. Therefore, we conducted a review on how contrast enhancement on MRI has been used to assess BCSFB permeability (Chapter 6). Post-contrast gadolinium enhancement in the CSF has been used to indicate BCSFB disruption (36-38) and new 
techniques are being developed, for instance imaging sequences highly sensitive to the CSF (39-41) or the use of other contrast agents than gadolinium (42-45). By further promoting these sorts of developments, we believe much progress can still be made in the assessment of BCSFB integrity.

\subsection{A broader perspective}

A previous study also found an association between BBB leakage and age, and demonstrated that BBB leakage was significantly higher in individuals with mild cognitive impairment compared to normal controls (8). Remarkably, these relations were only found for BBB leakage in the hippocampus, but not for leakage in the white matter or cortical grey matter. The authors suggest that, in normal aging, BBB disruption possibly begins in the hippocampus and may subsequently contribute to age-related cognitive impairment. However, we could only find an association with normal agerelated cognitive decline for $\mathrm{BBB}$ disruption in the cerebral white matter, and not in the hippocampus. Our results therefore do not support the previous suggestion that early BBB leakage beginning in the hippocampus contributes to cognitive impairment in normal aging.

In a later study, the same group found higher BBB leakage in the hippocampus of individuals with early cognitive dysfunction compared to healthy controls (15), and they elaborated on this finding by demonstrating that cognitive impairment could especially be linked to BBB leakage in the hippocampus among carriers of the $\varepsilon 4$ allelle of the apolipoprotein E gene (APOE4) (46). The APOE4 allele greatly increases risk of a neurodegenerative disorder, especially AD (47). Thus, BBB disruption in the hippocampus might be more involved in pathological aging. This might explain why the current study, focusing on successful aging, obtained a different result. Another previous study not only investigated normal controls, but also individuals with mild cognitive impairment and $\mathrm{AD}$ (48). This study found no significant relation between BBB disruption in the hippocampus and cognitive performance, and the authors suggest that, at a later stage of neurodegeneration, the effect of microvascular damage on cognitive performance might be negligible compared to the effect of neurodegenerative processes. This study also did not find a significant relation between BBB disruption in the white or grey matter and memory performance. The discrepancy with our results could be due to 
the use of longitudinal cognitive data in our study and could indicate that the influence of BBB disruption on cognitive performance is best visible in the long-term.

\subsection{Next steps in barrier imaging}

To confirm our findings, longitudinal data should be collected to investigate whether BBB leakage increases as a person ages and how this increase relates to other types of brain pathology and whether it is paired with a change in cognitive function. Only a longitudinal design will also allow to comment on causality and uncover whether BBB disruption precedes these other changes. For instance, white matter pathology might damage the vessel walls and generate $\mathrm{BBB}$ disruption. However, not only white matter hyperintensities (WMHs), but also their nearby normal-appearing white matter has been demonstrated to be more permeable, which disputes this notion and could mean that BBB disruption comes first in the pathological cascade and triggers white matter pathology (49-51). With the BBB leakage rate only being obtained at the end of the 12year interval, one cannot make inferences about causal relations, as persons with stronger BBB disruption and cognitive decline may have similar underlying origins, for instance neuroinflammation or cSVD, which have both been implicated in neurodegeneration and cognitive impairment (52-55).

Using the dual-time resolution DCE MRI technique to detect substantial contrast transfer in the BBB-lacking CVOs (26), was not only a way to validate the technique, but can also give rise to subsequent research, as we for the first time introduced DCE MRI as a possible method to investigate CVO characteristics. The CVOs are often referred to as 'windows of the brain', and pathogens may enter through these windows and trigger an inflammatory response (27). More advanced ways to assess CVO permeability might eventually help blocking entrance of pathogens, determine factors that disturb homeostasis and possibly contribute to initiatives to overcome neurodegenerative disorders.

An important future study direction can be found in efforts to measure glymphatic clearance using imaging. Imaging brain fluid dynamics may help in understanding the pathogenesis of glymphatic dysfunction (56). A measure of glymphatic clearance might be used to identify those at risk of $\mathrm{AD}$ or evaluate disease progression, and mapping the clearance kinetics may serve to monitor how a person responds to treatment (57). Future attempts to measure activity in the glymphatic system could for instance focus on 
quantitative imaging of CSF composition and flow through the perivascular space, the starting point of the glymphatic system. The imaging technique heavily $\mathrm{T}_{2}$-weighted fluid attenuated inversion recovery (hT2w-FLAIR) is highly sensitive to low gadolinium concentrations in the CSF and with this technique, researchers were for the first time able to detect signal enhancement in the perivascular space of the human brain, several hours after gadolinium administration (58). Diffusion tensor image analysis along the perivascular space (DTI-ALPS) can be used to measure water diffusivity along the perivascular space in the human brain (59). Lower diffusivity along the perivascular space could indicate less activity in the glymphatic system.

Besides BBB permeability, future studies could focus on BCSFB permeability and its relation to age-related brain pathology and neurodegeneration, as also for the BCSFB, age-associated damage can disrupt balance in the internal milieu and contribute to the neurodegenerative cascade (34). A promising technique to detect CSF enhancement in healthy individuals is for instance the previously mentioned hT2w-FLAIR, more sensitive to the CSF and able to detect lower gadolinium concentrations. Three-dimensional-real inversion recovery (3D-real IR) could also be promising, which uses phase-sensitive reconstruction that takes into account the negative magnetisation of CSF and can better distinguish between fluid compartments and tissue types (39-41). Other contrast agents than gadolinium-based contrast agents are also being considered. Manganese ions, for example, can better visualize neural connectivity and cellular integrity and accumulate in the choroid plexus, the site of the BCSFB $(42,43)$. Also, in animal studies, very small superparamagnetic iron oxide particles (VSOPs) are used as contrast agent, as these particles can be used for cell labelling through phagocytosis and highlight barrier disruptions (44). Europium-doped VSOPs (eu-VSOPs) even have fluorescence in addition to superparamagnetic properties, and can be used not only for contrast-enhanced MRI, but also for fluorescence microscopy and immunostaining (45).

\subsection{Potential limitations}

Ideally, BBB leakage would have been measured at the moment of the first cognitive testing, so we would have been able to investigate whether baseline BBB disruption predicts future cognitive decline. However, we considered the current design the best alternative, as 12 years ago, imaging techniques were not yet sensitive enough for the subtle leakage values in normal aging. The DCE MRI technique still has its limitations even 
now. The accuracy of BBB permeability measurements depends on determination of the vascular input function (VIF). Due to high blood velocity, the depiction of the arteries is susceptible to flow artifacts, so that the VIF is generally obtained in the superior sagittal sinus instead. Contrast concentration in the superior sagittal sinus may not represent actual input, as this is the site where contrast agent leaves the brain. However, the location of the superior sagittal sinus is easily identifiable and its depiction is less prone to partial volume or inflow artefacts $(60,61)$. Another potential issue that comes with measuring BBB permeability per voxel, is that with subtle leakage and low permeability rates, noise can lead to negative numerical values of leakage. A histogram of the leakage rates for all voxels indeed contains negative values, and since BBB leakage cannot be negative, these values can be assumed to be due to noise. When measuring BBB permeability per voxel in our studies, the negative part of the histogram representing noise was mirrored onto the positive part and subsequently subtracted from the histogram, as a way of noise correction and dealing with negative leakage values (60). A final disadvantage of the DCE MRI technique is that results from different sites cannot easily be compared to one another due to differences in method, for instance MRI sequence, pharmacokinetic model selection or data analysis (17). For the future, it would be beneficial to design a method that can be uniformly applied across sites.

Our participants have participated in MAAS for 25 years (18). Participants who are willing and able to participate over such a long period of time are more likely to represent those with an above-average health condition, and might not be an accurate representation of the population. Still, we were able to find significantly higher BBB leakage with older age and significantly worse cognitive performance with higher leakage rates even within this functionally healthy subsample, and these effects will possibly be even stronger in a more diverse group. Therefore, we expect our findings to be relevant to the general aging population.

\subsection{Clinical implications}

Maintaining homeostasis in the CNS, and thereby preventing ion fluctuations, is important for synaptic, and thereby neuronal functioning. Homeostasis is dependent on the dynamics between the blood, CSF and ISF, which are collectively termed the neurofluids (62). Several disorders have been associated with a disturbance in neurofluid dynamics, such as sleep disorders (63), AD (64), Parkinson's disease (65), traumatic 
brain injury (66) and stroke (67), and these disorders can be grouped under the term CNS interstitial fluidopathies (68). A disturbance in neurofluid dynamics can have various consequences, such as accumulation of toxic waste products, impaired immune function or impaired signal transmission, so that CNS interstitial fluidopathies can have various clinical manifestations and symptoms. Although these disorders are not usually grouped together, classifying them as CNS interstitial fluidopathies recognises that they could share an underlying pathology, which could be helpful for treatment and prevention efforts $(68,69)$. A disturbance in neurofluid dynamics can be caused by BBB disruption, BCSFB disruption or potentially glymphatic dysfunction, and these processes likely play an important role in CNS interstitial fluidopathies (68). Since the introduction of the glymphatic system (29), efforts are being made to use MRI to assess glymphatic function, CSF/ISF dynamics or flow in the perivascular and interstitial spaces. Using imaging to obtain information on mechanisms related to neurofluid composition and dynamics could increase understanding of the underlying pathophysiology of CNS interstitial fluidopathies, and might even help in the development of novel common treatment options $(68,69)$.

As the integrity of the cerebral microvasculature seems to be an important factor in the development of a number of disorders, prevention strategies should focus on promoting neurovascular health. Studies are often aimed at the removal of neurotoxic proteins, but also identification of methods to maintain BBB integrity already at an early stage should be a future research focus. For instance, there has been promising work on restoring $\mathrm{NAD}^{+}$levels, which was demonstrated to protect the integrity of the cerebral microvasculature (70-74), and could even be relevant for the maintenance of cognitive functioning. It would also be interesting to investigate whether reducing vascular risk, by for instance life-style adaptations, mitigates age-related cognitive decline by reducing BBB disruption. Ventures such as these might eventually help increase chances of successful aging $(2,75)$.

Another factor that could influence the risk of BBB damage is infection with the severe acute respiratory syndrome coronavirus 2 (SARS-CoV-2), the virus which causes human coronavirus disease 2019 (COVID-19). Endothelial cells express the angiotensin converting enzyme 2 (ACE2), to which SARS-CoV-2 can bind (76), and the virus can gain access to the vascular and neuronal tissue by infecting the endothelial cells (77). 
Moreover, SARS-CoV-2 can infect T-lymphocytes, cause systemic inflammation and increase BBB permeability $(77,78)$, so that infected T-lymphocytes, cytokines and the virus itself can pass the BBB and enter the CNS (79). SARS-CoV-2 can then interact with vascular and neuronal ACE2 receptors, leading to a cycle of viral budding and neuronal damage $(80,81)$. Longitudinal neurological assessment of individuals who recovered from COVID-19 should be conducted to fully understand the effects of SARS-CoV-2 on the CNS (77). SARS-CoV-2 may not only increase the risk of neurovascular events such as haemorrhagic stroke, encephalopathy or encephalitis (82), but may also lead to subtle damage to the neurovasculature, such as widespread BBB breakdown $(77,80)$. Small neurovascular alterations such as these might over time lead to neurovascular dysfunction and trigger age-related brain pathology or possibly age-related cognitive decline. However, much is still unknown about COVID-19 and its effect on the CNS, and we can only speculate about the possible long-term neurovascular consequences. Nevertheless, we think it is worth considering, especially concerning the large scale on which people all over the world have been infected by SARS-CoV-2.

\section{7. $\quad$ Future goals}

We expect to be able to use BBB integrity as an indicator of risk of age-related neurological and cognitive impairment. BBB integrity was not only associated with agerelated disorders, but also normal aging and normal age-related cognitive decline, supporting the notion that it could initiate age-related brain pathology. If methods to measure BBB leakage do not only become more sensitive, but also easier to implement, we might be able to establish whether someone's BBB permeability is reason for concern at an early stage, before it might trigger the pathological cascade causing neurodegeneration. Ideally, methods to promote BBB integrity will be further developed, for instance restoring $\mathrm{NAD}^{+}$levels or life-style adaptations reducing vascular risk, so that we can timely intervene to counter BBB breakdown and perhaps prevent or further defer neuropathology.

However, before using BBB integrity as an indicator of risk of age-related impairment, the relation between BBB disruption and age-related brain pathology and cognitive decline must be further detailed. Also, to assess this risk at an early stage, the method to measure BBB leakage must be so far developed to allow for large scale implementation. Moreover, as methods to promote BBB integrity are still being 
investigated and have not been validated yet, the desirability of such early stage detection is questionable. Determining at an early phase whether someone is at risk of pathology later in life, without offering any means to counteract it, raises ethical concerns.

\subsection{Conclusion}

We have demonstrated that BBB disruption is already associated with age and cognitive decline in neurologically and cognitively healthy individuals, which supports the notion of BBB disruption as an early event and a common trigger of age-related brain pathology. Moreover, it suggests that BBB disruption is an underlying contributor to normal agerelated neurological and cognitive decline, and might in the future be a target to promote successful aging. Longitudinal studies are needed to establish the causal relations, but promoting BBB integrity seems a promising future study direction. Meanwhile the development of new MRI techniques to measure CVO characteristics, glymphatic function or BCSFB integrity could inspire new imaging initiatives to investigate how these processes are involved in maintaining balance in the CNS, and how this relates to aging, neurodegeneration and cognitive function.

\subsection{References}

1. He W, Goodkind D, Kowal PR. An aging world: 2015. 2016.

2. Deckers K, van Boxtel MP, Schiepers OJ, de Vugt M, Muñoz Sánchez JL, Anstey KJ, et al. Target risk factors for dementia prevention: a systematic review and Delphi consensus study on the evidence from observational studies. Int J Geriatr Psychiatry. 2015;30(3):234-46.

3. Choi JY, Morris JC, Hsu CY. Aging and cerebrovascular disease. Neurologic clinics. 1998;16(3):687711.

4. Mooradian AD. Potential mechanisms of the age-related changes in the blood-brain barrier. Neurobiology of Aging. 1994;15(6):751-5.

5. Farrall AJ, Wardlaw JM. Blood-brain barrier: ageing and microvascular disease-systematic review and meta-analysis. Neurobiology of aging. 2009;30(3):337-52.

6. Zlokovic BV. The blood-brain barrier in health and chronic neurodegenerative disorders. Neuron. 2008;57(2):178-201.

7. Iturria-Medina Y, Sotero RC, Toussaint PJ, Mateos-Pérez JM, Evans AC, The Alzheimer's Disease Neuroimaging I, et al. Early role of vascular dysregulation on late-onset Alzheimer's disease based on multifactorial data-driven analysis. Nature Communications. 2016;7:11934.

8. Montagne A, Barnes SR, Sweeney MD, Halliday MR, Sagare AP, Zhao Z, et al. Blood-brain barrier breakdown in the aging human hippocampus. Neuron. 2015;85(2):296-302. 


\section{Chapter 7}

9. Montagne A, Nation DA, Pa J, Sweeney MD, Toga AW, Zlokovic BV. Brain imaging of neurovascular dysfunction in Alzheimer's disease. Acta neuropathologica. 2016;131(5):687-707.

10. van de Haar HJ, Burgmans S, Jansen JF, van Osch MJ, van Buchem MA, Muller M, et al. Blood-brain barrier leakage in patients with early Alzheimer disease. Radiology. 2016;281(2):527-35.

11. Burgmans S, van de Haar HJ, Verhey FR, Backes WH. Amyloid- $\beta$ interacts with blood-brain barrier function in dementia: a systematic review. Journal of Alzheimer's Disease. 2013;35(4):859-73.

12. Sironi L, Guerrini U, Tremoli E, Miller I, Gelosa P, Lascialfari A, et al. Analysis of pathological events at the onset of brain damage in stroke-prone rats: A proteomics and magnetic resonance imaging approach. Journal of neuroscience research. 2004;78(1):115-22.

13. Glisky EL. Changes in cognitive function in human aging. Brain aging: models, methods, and mechanisms. 2007:3-20.

14. Rapp PR, Amaral DG. Individual differences in the cognitive and neurobiological consequences of normal aging. Trends in neurosciences. 1992;15(9):340-5.

15. Nation DA, Sweeney MD, Montagne A, Sagare AP, D’Orazio LM, Pachicano M, et al. Blood-brain barrier breakdown is an early biomarker of human cognitive dysfunction. Nature Medicine. 2019.

16. van de Haar HJ, Burgmans S, Hofman PA, Verhey FR, Jansen JF, Backes WH. Blood-brain barrier impairment in dementia: current and future in vivo assessments. Neuroscience \& Biobehavioral Reviews. 2014.

17. Raja R, Rosenberg GA, Caprihan A. MRI measurements of Blood-Brain Barrier function in dementia: A review of recent studies. Neuropharmacology. 2018;134:259-71.

18. Jolles J, Houx P, Van Boxtel M, Ponds R. Maastricht aging study: Determinants of cognitive aging: Neuropsych Publishers Maastricht; 1995.

19. Andrews-Hanna JR, Snyder AZ, Vincent JL, Lustig C, Head D, Raichle Marcus E, et al. Disruption of Large-Scale Brain Systems in Advanced Aging. Neuron. 2007;56(5):924-35.

20. Casey BJ, Tottenham N, Liston C, Durston S. Imaging the developing brain: what have we learned about cognitive development? Trends in Cognitive Sciences. 2005;9(3):104-10.

21. Martin AJ, Friston KJ, Colebatch JG, Frackowiak RS. Decreases in regional cerebral blood flow with normal aging. Journal of Cerebral Blood Flow \& Metabolism. 1991;11(4):684-9.

22. Fjell AM, McEvoy L, Holland D, Dale AM, Walhovd KB. Brain Changes in Older Adults at Very Low Risk for Alzheimer's Disease. The Journal of Neuroscience. 2013;33(19):8237-42.

23. Eichenbaum H. Hippocampus: cognitive processes and neural representations that underlie declarative memory. Neuron. 2004;44(1):109-20.

24. Eichenbaum H. A cortical-hippocampal system for declarative memory. Nature Reviews Neuroscience. 2000;1(1):41-50.

25. Daigneault S, Braun CM. Working memory and the self-ordered pointing task: Further evidence of early prefrontal decline in normal aging. Journal of clinical and Experimental Neuropsychology. 1993;15(6):881-95.

26. Duvernoy HM, Risold P-Y. The circumventricular organs: an atlas of comparative anatomy and vascularization. Brain research reviews. 2007;56(1):119-47. 
27. Sisó S, Jeffrey M, González L. Sensory circumventricular organs in health and disease. Acta neuropathologica. 2010;120(6):689-705.

28. Shibata M, Yamada S, Kumar SR, Calero M, Bading J, Frangione B, et al. Clearance of Alzheimer's amyloid- $\beta$ 1-40 peptide from brain by LDL receptor-related protein-1 at the blood-brain barrier. The Journal of clinical investigation. 2000;106(12):1489-99.

29. Iliff JJ, Wang M, Liao Y, Plogg BA, Peng W, Gundersen GA, et al. A paravascular pathway facilitates CSF flow through the brain parenchyma and the clearance of interstitial solutes, including amyloid $\beta$. Science translational medicine. 2012;4(147):147ra11-ra11.

30. Kress BT, Iliff JJ, Xia M, Wang M, Wei HS, Zeppenfeld D, et al. Impairment of paravascular clearance pathways in the aging brain. Annals of neurology. 2014;76(6):845-61.

31. Xie L, Kang H, Xu Q, Chen MJ, Liao Y, Thiyagarajan M, et al. Sleep drives metabolite clearance from the adult brain. science. 2013;342(6156):373-7.

32. Tarasoff-Conway JM, Carare RO, Osorio RS, Glodzik L, Butler T, Fieremans E, et al. Clearance systems in the brain [mdash] implications for Alzheimer disease. Nature Reviews Neurology. 2015;11(8):457-70.

33. Hladky SB, Barrand MA. Metabolite Clearance During Wakefulness and Sleep. Handbook of experimental pharmacology. 2017.

34. Johanson CE. Chapter 11 - Choroid Plexus-Cerebrospinal Fluid Transport Dynamics: Support of Brain Health and a Role in Neurotherapeutics. In: Conn PM, editor. Conn's Translational Neuroscience. San Diego: Academic Press; 2017. p. 233-61.

35. Balusu S, Brkic M, Libert C, Vandenbroucke RE. The choroid plexus-cerebrospinal fluid interface in Alzheimer's disease: more than just a barrier. Neural regeneration research. 2016;11(4):534.

36. Renú A, Laredo C, Lopez-Rueda A, Llull L, Tudela R, San-Roman L, et al. Vessel Wall enhancement and blood-cerebrospinal fluid barrier disruption after mechanical thrombectomy in acute ischemic stroke. Stroke. 2017;48(3):651-7.

37. Kim H, Lim Y-M, Kim G, Lee E-J, Lee JH, Kim HW, et al. Choroid plexus changes on magnetic resonance imaging in multiple sclerosis and neuromyelitis optica spectrum disorder. Journal of the Neurological Sciences. 2020:116904.

38. Eide PK, Valnes LM, Pripp AH, Mardal K-A, Ringstad G. Delayed clearance of cerebrospinal fluid tracer from choroid plexus in idiopathic normal pressure hydrocephalus. Journal of Cerebral Blood Flow \& Metabolism. 2019:0271678X19874790.

39. Jost G, Lenhard DC, Sieber MA, Lohrke J, Frenzel T, Pietsch H. Signal increase on unenhanced T1weighted images in the rat brain after repeated, extended doses of gadolinium-based contrast agents: comparison of linear and macrocyclic agents. Investigative radiology. 2016;51(2):83.

40. Deike-Hofmann K, Reuter J, Haase R, Paech D, Gnirs R, Bickelhaupt S, et al. Glymphatic pathway of gadolinium-based contrast agents through the brain: overlooked and misinterpreted. Investigative radiology. 2019;54(4):229-37.

41. Ohashi T, Naganawa S, Iwata S, Kuno K. Distribution of Gadolinium-based Contrast Agent after Leaking into the Cerebrospinal Fluid: Comparison between the Cerebral Cisterns and the Lateral Ventricles. Magnetic Resonance in Medical Sciences. 2020:mp. 2020-0016. 


\section{Chapter 7}

42. Sudarshana DM, Nair G, Dwyer JT, Dewey B, Steele SU, Suto DJ, et al. Manganese-enhanced MRI of the brain in healthy volunteers. American Journal of Neuroradiology. 2019;40(8):1309-16.

43. Aoki I, Wu Y-JL, Silva AC, Lynch RM, Koretsky AP. In vivo detection of neuroarchitecture in the rodent brain using manganese-enhanced MRI. Neuroimage. 2004;22(3):1046-59.

44. Millward JM, Schnorr J, Taupitz M, Wagner S, Wuerfel JT, Infante-Duarte C. Iron oxide magnetic nanoparticles highlight early involvement of the choroid plexus in central nervous system inflammation. 2013.

45. Millward JM, Ariza de Schellenberger A, Berndt D, Hanke-Vela L, Schellenberger E, Waiczies S, et al. Application of Europium-Doped Very Small Iron Oxide Nanoparticles to Visualize Neuroinflammation with MRI and Fluorescence Microscopy. Neuroscience. 2019;403:136-44.

46. Montagne A, Nation DA, Sagare AP, Barisano G, Sweeney MD, Chakhoyan A, et al. APOE4 leads to blood-brain barrier dysfunction predicting cognitive decline. Nature. 2020;581(7806):71-6.

47. Yamazaki Y, Zhao N, Caulfield TR, Liu C-C, Bu G. Apolipoprotein E and Alzheimer disease: pathobiology and targeting strategies. Nature Reviews Neurology. 2019;15(9):501-18.

48. Freeze WM, Jacobs HI, de Jong JJ, Verheggen IC, Gronenschild EH, Palm WM, et al. White matter hyperintensities mediate the association between blood-brain barrier leakage and information processing speed. Neurobiology of aging. 2020;85:113-22.

49. Riphagen JM, Gronenschild EHBM, Salat DH, Freeze WM, Ivanov D, Clerx L, et al. Shades of white: diffusion properties of T1- and FLAIR-defined white matter signal abnormalities differ in stages from cognitively normal to dementia. Neurobiology of Aging. 2018;68:48-58.

50. Wong SM, Jansen JF, Zhang CE, Hoff EI, Staals J, van Oostenbrugge RJ, et al. Blood-brain barrier impairment and hypoperfusion are linked in cerebral small vessel disease. Neurology. 2019;92(15):e1669e77.

51. Hainsworth A. White matter lesions in cerebral small vessel disease: Underperfusion or leaky vessels? Neurology. 2019;92:10.1212/WNL.0000000000007258.

52. Kazakos EI, Kountouras J, Polyzos SA, Deretzi G. Novel aspects of defensins' involvement in virusinduced autoimmunity in the central nervous system. Medical Hypotheses. 2017;102(Supplement C):33-6. 53. Li Y, Li M, Zuo L, Shi Q, Qin W, Yang L, et al. Compromised Blood-Brain Barrier Integrity Is Associated With Total Magnetic Resonance Imaging Burden of Cerebral Small Vessel Disease. Front Neurol. 2018;9:221.

54. Shlosberg D, Benifla M, Kaufer D, Friedman A. Blood-brain barrier breakdown as a therapeutic target in traumatic brain injury. Nature Reviews Neurology. 2010;6(7):393-403.

55. Debette S, Beiser A, DeCarli C, Au R, Himali JJ, Kelly-Hayes M, et al. Association of MRI markers of vascular brain injury with incident stroke, mild cognitive impairment, dementia, and mortality: the Framingham Offspring Study. Stroke. 2010;41(4):600-6.

56. Wostyn P, Van Dam D, Audenaert K, Killer HE, De Deyn PP, De Groot V. A new glaucoma hypothesis: a role of glymphatic system dysfunction. Fluids and Barriers of the CNS. 2015;12(1):1.

57. Nedergaard M. Garbage truck of the brain. Science. 2013;340(6140):1529-30.

58. Naganawa S, Nakane T, Kawai H, Taoka T. Gd-based contrast enhancement of the perivascular spaces in the basal ganglia. Magnetic Resonance in Medical Sciences. 2017;16(1):61-5. 
59. Taoka T, Masutani Y, Kawai H, Nakane T, Matsuoka K, Yasuno F, et al. Evaluation of glymphatic system activity with the diffusion MR technique: diffusion tensor image analysis along the perivascular space (DTI-ALPS) in Alzheimer's disease cases. Japanese journal of radiology. 2017;35(4):172-8.

60. van de Haar HJ, Jansen JFA, Jeukens CRLPN, Burgmans S, Buchem MA, Muller M, et al. Subtle bloodbrain barrier leakage rate and spatial extent: Considerations for dynamic contrast-enhanced MRI. Medical Physics. 2017;44(8):4112-25.

61. Lavini C, Verhoeff JJC. Reproducibility of the gadolinium concentration measurements and of the fitting parameters of the vascular input function in the superior sagittal sinus in a patient population. Magnetic Resonance Imaging. 2010;28(10):1420-30.

62. Agarwal N, Contarino C, Toro EF. Neurofluids: A holistic approach to their physiology, interactive dynamics and clinical implications for neurological diseases. Veins and Lymphatics. 2019;8(3).

63. DiNuzzo M, Nedergaard M. Brain energetics during the sleep-wake cycle. Current opinion in neurobiology. 2017;47:65-72.

64. Schubert JJ, Veronese M, Marchitelli L, Bodini B, Tonietto M, Stankoff B, et al. Dynamic 11C-PiB PET shows cerebrospinal fluid flow alterations in Alzheimer disease and multiple sclerosis. Journal of Nuclear Medicine. 2019;60(10):1452-60.

65. Sundaram S, Hughes RL, Peterson E, Müller-Oehring EM, Bronte-Stewart HM, Poston KL, et al. Establishing a framework for neuropathological correlates and glymphatic system functioning in Parkinson's disease. Neuroscience \& Biobehavioral Reviews. 2019;103:305-15.

66. Christensen J, Wright DK, Yamakawa GR, Shultz SR, Mychasiuk R. Repetitive mild traumatic brain injury alters glymphatic clearance rates in limbic structures of adolescent female rats. Scientific reports. 2020;10(1):1-9.

67. Gaberel T, Gakuba C, Goulay R, De Lizarrondo SM, Hanouz J-L, Emery E, et al. Impaired Glymphatic Perfusion After Strokes Revealed by Contrast-Enhanced MRI. Stroke. 2014:STROKEAHA. 114.006617.

68. Taoka T, Naganawa S. Imaging for central nervous system (CNS) interstitial fluidopathy: disorders with impaired interstitial fluid dynamics. Japanese Journal of Radiology. 2020.

69. Nakashima T, Sone M, Teranishi M, Yoshida T, Terasaki H, Kondo M, et al. A perspective from magnetic resonance imaging findings of the inner ear: relationships among cerebrospinal, ocular and inner ear fluids. Auris Nasus Larynx. 2012;39(4):345-55.

70. Csiszar A, Tarantini S, Yabluchanskiy A, Balasubramanian P, Kiss T, Farkas E, et al. Role of endothelial NAD+ deficiency in age-related vascular dysfunction. American Journal of Physiology-Heart and Circulatory Physiology. 2019;316(6):H1253-H66.

71. Kiss T, Balasubramanian P, Valcarcel-Ares MN, Tarantini S, Yabluchanskiy A, Csipo T, et al. Nicotinamide mononucleotide (NMN) treatment attenuates oxidative stress and rescues angiogenic capacity in aged cerebromicrovascular endothelial cells: a potential mechanism for the prevention of vascular cognitive impairment. GeroScience. 2019;41(5):619-30.

72. Tarantini S, Yabluchanskiy A, Csipo T, Fulop G, Kiss T, Balasubramanian P, et al. Treatment with the poly (ADP-ribose) polymerase inhibitor PJ-34 improves cerebromicrovascular endothelial function, neurovascular coupling responses and cognitive performance in aged mice, supporting the NAD+ depletion hypothesis of neurovascular aging. GeroScience. 2019;41(5):533-42. 


\section{Chapter 7}

73. Mills KF, Yoshida S, Stein LR, Grozio A, Kubota S, Sasaki Y, et al. Long-term administration of nicotinamide mononucleotide mitigates age-associated physiological decline in mice. Cell metabolism. 2016;24(6):795-806.

74. Zhang H, Ryu D, Wu Y, Gariani K, Wang X, Luan P, et al. NAD+ repletion improves mitochondrial and stem cell function and enhances life span in mice. Science. 2016;352(6292):1436-43.

75. Livingston G, Huntley J, Sommerlad A, Ames D, Ballard C, Banerjee S, et al. Dementia prevention, intervention, and care: 2020 report of the Lancet Commission. The Lancet. 2020;396(10248):413-46.

76. Hoffmann M, Kleine-Weber H, Schroeder S, Krüger N, Herrler T, Erichsen S, et al. SARS-CoV-2 Cell Entry Depends on ACE2 and TMPRSS2 and Is Blocked by a Clinically Proven Protease Inhibitor. Cell. 2020;181(2):271-80.e8.

77. Zubair AS, McAlpine LS, Gardin T, Farhadian S, Kuruvilla DE, Spudich S. Neuropathogenesis and Neurologic Manifestations of the Coronaviruses in the Age of Coronavirus Disease 2019: A Review. JAMA Neurology. 2020.

78. Desforges M, Le Coupanec A, Brison É, Meessen-Pinard M, Talbot PJ. Neuroinvasive and neurotropic human respiratory coronaviruses: potential neurovirulent agents in humans. Infectious Diseases and Nanomedicine I: Springer; 2014. p. 75-96.

79. Sankowski R, Mader S, Valdés-Ferrer SI. Systemic Inflammation and the Brain: Novel Roles of Genetic, Molecular, and Environmental Cues as Drivers of Neurodegeneration. Frontiers in Cellular Neuroscience. 2015;9(28).

80. Baig AM, Khaleeq A, Ali U, Syeda H. Evidence of the COVID-19 Virus Targeting the CNS: Tissue Distribution, Host-Virus Interaction, and Proposed Neurotropic Mechanisms. ACS Chemical Neuroscience. 2020;11(7):995-8.

81. Netland J, Meyerholz DK, Moore S, Cassell M, Perlman S. Severe Acute Respiratory Syndrome Coronavirus Infection Causes Neuronal Death in the Absence of Encephalitis in Mice Transgenic for Human ACE2. Journal of Virology. 2008;82(15):7264-75.

82. Wu Y, Xu X, Chen Z, Duan J, Hashimoto K, Yang L, et al. Nervous system involvement after infection with COVID-19 and other coronaviruses. Brain, behavior, and immunity. 2020. 




\section{Summary}

Neurovascular health is one of the factors that influences whether someone ages successfully and depends, among others, on the integrity of the blood-brain barrier (BBB). BBB disruption can disturb homeostasis in the brain tissue, which is detrimental to neuronal functioning. It may also impair the clearance of the neurotoxic biomolecules, for instance the amyloid- $\beta$ protein $(A \beta)$, which accumulates in Alzheimer's disease. BBB disruption might be a common trigger for age-related brain pathology and an underlying mechanism of the decline that accompanies aging, even before overt age-related brain pathology emerges. Interest in BBB disruption has increased due to the development of more sensitive in vivo imaging techniques such as dynamic contrast-enhanced magnetic resonance imaging (DCE MRI), which has for instance detected subtle BBB leakage in dementia. In DCE MRI, a contrast agent is intravenously injected during scanning and its biological distribution is imaged over time. In a healthy brain, the contrast agent remains in the blood stream. In brains with an impaired BBB, however, contrast material transfers from blood to brain tissue and the transfer constant then represents the amount of BBB leakage. However, DCE MRI studies looking into BBB disruption and its relation to normal aging and age-related cognitive decline have not been conducted. Therefore, this thesis aimed to investigate whether BBB leakage was significantly associated with older age and decline in cognitive performance in a healthy sample. Participants without major cognitive or neurological impairment were selected from the longitudinal Maastricht Aging Study (MAAS). We conducted neuropsychological assessment and used the MAAS data to calculate decline in cognitive performance over a 12-year period, and we performed dual-time resolution DCE MRI, which was sensitive enough to detect even the subtle leakage values found in normal aging individuals.

In Chapter 1, we provide an introduction and first discuss the importance of investigating what factors determine successful aging. We then discuss how neurovascular dysfunction and BBB disruption may contribute to age-related pathology and cognitive decline, by disrupting homeostasis in the brain tissue and triggering pathological processes, and impairing $A \beta$ clearance. Also, we discuss the DCE MRI technique and how this technique has enabled us to measure in vivo even subtle BBB leakage. 
Chapter 2 contains our DCE MRI study to investigate whether older age was associated with more BBB disruption, even in cognitively and neurologically healthy individuals, and whether this association was stronger for brain regions sensitive to agerelated impairment. We found a higher BBB leakage rate in the total cerebral white and grey matter for older individuals. Moreover, BBB disruption increases with age especially in those regions known to be most vulnerable to age-related deterioration, namely the brain regions involved in higher-order cognitive functions. Our results provide evidence that BBB breakdown may underlie normal age-related deterioration.

Chapter 3 contains our DCE MRI study to investigate whether larger BBB disruption was paired with stronger normal age-related cognitive decline. We found a significant association between BBB leakage in the white matter and 12-year decline in delayed recall score, which can be considered a measure of memory retrieval. Our results support the notion that white matter BBB disruption may be a contributing mechanism to cognitive aging, seemingly affecting memory retrieval first.

In Chapter 4, we discuss our feasibility study aiming to validate the DCE MRI technique by measuring contrast transfer in the circumventricular organs (CVOs). These structures regulate communication between the blood, cerebrospinal fluid (CSF) and brain and do not have a BBB. We indeed found significantly positive transfer constants in the CVOs, higher than in the normal-appearing brain matter and highest for the secretory CVOs. Our results introduce DCE MRI as a possible method to assess CVO permeability characteristics and how pathogens can potentially enter the brain via these structures.

In Chapter 5, a review study was conducted attempting to resolve how waste clearance from the brain through BBB transport relates to the recently discovered glymphatic system. The glymphatic system is a clearance mechanism using the microcirculation of the CSF, in which the CSF is exchanged with interstitial fluid to clear solutes from the interstitial space. BBB transport and glymphatic clearance likely serve complementary roles with partially overlapping mechanisms to provide a well-balanced neuronal environment. Properly functioning waste clearance systems protect against the accumulation of toxic waste products such as $A \beta$, and may delay or even prevent the onset of neurodegenerative disorders such as AD.

In Chapter 6, a review study was conducted on the use of contrast-enhancement on MRI to assess blood-CSF barrier (BCSFB) permeability. The BCSFB is involved in the composition of the CSF and thus the maintenance of homeostasis in the central nervous 
system. While the relation between BBB leakage, aging and neurodegeneration is being extensively studied using contrast-enhanced MRI, the use of this technique to investigate BCSFB leakage in relation to age-related neurodegeneration has received little attention. Post-contrast gadolinium enhancement in the CSF has been used to indicate BCSFB disruption and new techniques are being developed, for instance imaging sequences highly sensitive to the CSF or the use of other contrast agents than gadolinium compounds.

Finally, in Chapter 7, we provide a general discussion on the meaning and implications of our results. We believe they provide evidence that BBB disruption may be an underlying mechanism of normal age-related decline and contributes to cognitive aging. We suggest that BBB disruption may in the future become a target for efforts to promote successful aging, especially those aiming to promote vascular health. However, further research is necessary, for instance to establish causal relations. The current studies demonstrate the suitability of the DCE MRI technique to measure in vivo subtle BBB leakage. We also suggest new study directions and imaging developments, such as methods to measure glymphatic function or BCSFB integrity. 



\section{Impact paragraph}

\section{Main findings}

The main goal of this thesis was to see whether we could support the notion that bloodbrain barrier (BBB) disruption may be an underlying mechanism of normal age-related decline and a contributing factor to cognitive aging. Therefore, we investigated whether older individuals have more BBB leakage, even when healthy, and whether higher BBB leakage is then paired with more normal age-related cognitive decline. To detect the subtle leakage values that occur in normal aging, we used dynamic contrast-enhanced magnetic resonance imaging (DCE MRI). In this technique, a contrast agent is injected into the blood stream during scanning, so that the amount of contrast spreading from the blood to the brain can be measured

We found that BBB leakage in the white matter was significantly higher in older individuals. The association between BBB disruption and age was strongest in those brain regions known to be especially vulnerable to age-related deterioration, namely the brain regions involved in higher-order cognitive functions. Moreover, higher BBB leakage in the white matter was paired with significantly stronger decline in memory retrieval. These relations already being detected in people who age without overt cognitive or neurological impairment, supports the notion that BBB disruption may be an initiating factor of age-related pathology and one of the age-related brain changes contributing to cognitive impairment in older individuals.

To validate the technique, we applied DCE MRI to the circumventricular organs (CVOs). These small structures do not have a BBB, as their main function is to regulate the communication between the blood, cerebrospinal fluid (CSF) and brain. We could detect significantly positive contrast transfer in the CVOs, and introduced dynamic contrast-enhanced MRI as a promising method to investigate CVO permeability characteristics to better understand how pathogens could enter the brain through these structures.

To elaborate on other systems important for homeostasis in the central nervous system (CNS) and potentially involved in age-related impairment, namely the glymphatic system and the blood-CSF barrier (BCSFB), we conducted two review studies. The glymphatic system is a waste clearance system in which CSF is exchanged with interstitial 
Impact paragraph

fluid to clear solutes from the interstitial space. In the first review study, we concluded that BBB transport and glymphatic clearance are complementary clearance systems with partially overlapping mechanisms to provide a well-balanced neural environment. The second review study showed that contrast-enhanced MRI can also be used to measure BCSFB disruption, while this has received less attention than measuring BBB disruption. The BCSFB determines the composition of the CSF and also influences homeostasis in the CNS. New techniques to measure glymphatic clearance or BCSFB leakage are being developed, for instance techniques that can detect even very low contrast concentrations in the CSF.

\section{Relevance}

People do not only want to reach older ages, they want to reach these ages in a healthy manner. However, many people fear they will experience declining mental health, in particular cognitive decline, as they get older. This emphasizes the relevance of determining what factors influence how much cognitive decline someone will experience over time. We have now obtained evidence that there is indeed a role for the BBB, as people who experienced more cognitive decline over the years were shown to have more BBB leakage. BBB disruption seems to be present before the emergence of disease or disability or overt brain pathology, and could be a promising target for interventions aimed at improving vascular health and promoting successful aging.

For the time being, however, interventions that repair BBB leakage to prevent agerelated decline are under development, but not yet available. Still, indicating BBB disruption as contributor to cognitive aging has scientific relevance and can provide us with new study directions. We could for instance investigate BBB breakdown and cognitive changes over time, to obtain more information on the causal relations, or see whether health interventions that aim to promote successful brain aging are in part mediated by BBB disruption, and how this subsequently relates to cognitive decline. In the future, BBB leakage rate might even be used as a biological outcome measure to evaluate the effectiveness of such interventions. Moreover, our findings could also be an incentive to develop methods to prevent or reverse BBB damage.

BBB disruption is not only present in dementia, but already associated with cognitive decline in normal aging individuals, which implies that it may occur very early in the process of neurodegeneration. Alzheimer's disease and Alzheimer pathology used 
to be seen as separate from vascular dementia and vascular pathology. Lately, however, it has become understood that both forms of pathology are overlapping and additive or interacting processes and may have a common trigger. The BBB is part of the vasculature and BBB disruption is strongly involved in vascular pathology. Moreover, the BBB is important for the removal of the amyloid- $\beta$ protein from the brain, and accumulation of this protein is an important hallmark of Alzheimer's disease. Being an early event in neurodegeneration and being associated with both vascular and Alzheimer pathology, suggests that BBB disruption may possibly be a common trigger for age-related brain pathology.

Our findings have conceptual relevance and emphasize that knowledge on vascular mechanisms should be integrated with knowledge on Alzheimer mechanisms, as these mechanisms are not separate processes, but influence each other. Many intervention studies have primarily focused on removal of the amyloid- $\beta$ protein from the brain. Future studies, however, should also focus on barrier integrity and vascular health. The general public is mostly aware that vascular risk factors, such as high blood pressure, high fat diet, physical inactivity or high cholesterol, increase their risk of cardiovascular disease. It is much less known that, or how, these vascular risk factors can also influence our brain health. Although our specific findings should be further investigated and validated before being communicated to the general public, we have recently obtained a better general understanding of the link between vascular damage and brain pathology. The general public could be made more aware that risk factors for cardiovascular disease may also put them at risk of neurovascular disease and neurodegenerative disorders.

In a broader perspective, any disruption in barrier function or impairment in waste clearance system can disturb the chemical balance in the brain tissue, which can be very detrimental to neuronal functioning and lead to a variety of pathological processes, such as inflammation or hypoxia. Not only dementia is accompanied by such a disturbance in homeostasis, but conditions such as sleep disorders, traumatic brain injury and stroke are characterized by an imbalance in the brain fluid dynamics. Gaining more knowledge on barrier function during aging and neurodegeneration and finding new ways of measuring barrier function may be proven useful for a much wider range of conditions. 
Impact paragraph

\section{Target group}

First of all, our findings can be an incentive for further research and can therefore be important for other researchers, such as neuroscientists. The findings need to be further elucidated, for instance by establishing the causal relations between BBB disruption, other types of brain pathology and cognitive change. Furthermore, interventions to prevent or reverse $\mathrm{BBB}$ damage should be developed and investigated. These interventions can range from lifestyle changes to protect vascular integrity to biological interventions, such as restoring $\mathrm{NAD}^{+}$levels, which has been demonstrated to protect BBB integrity. Our findings can also be of interest to MR physicists. In particular, the BBB leakage measurement requires further standardization to design a method that can be uniformly applied across various research sites. The method also requires improvement of the detection sensitivity to very low concentrations of (extravasated) gadoliniumbased contrast agent. We have also mentioned the emergence of new techniques to image glymphatic clearance or BCSFB leakage. Further developing and implementing these techniques may offer exciting new research opportunities.

BBB disruption may become a valuable target for aging research. Not only can it lead to disturbance in homeostasis of the CNS and contribute to a wide range of disorders, it may also be an underlying mechanism of cognitive decline in normal aging individuals. Moreover, BBB disruption seems to occur before neurovascular pathology or other types of brain pathology become visible. While our findings specifically are more relevant to the scientific community than to the general aging population, the public could be made more aware that their vascular health, and lifestyle choices that affect their vascular health, may influence their chances of successful aging. In creating this kind of awareness lies an important task for the government, whose responsibility it is to advice and inform the general public through health campaigns.

\section{Dissemination activities}

Our findings have been spread throughout the scientific community by publications in scientific journals and presentations at scientific conferences (Cerebral Vascular Biology conference 2019, International Society for Magnetic Resonance Imaging conference 2020). They fit in a growing research interest into the role of vascular factors in neurodegeneration and support the notion that, besides accumulation of the amyloid- $\beta$ protein, studies into Alzheimer's disease should focus on neurovascular dysfunction. 
Recently, the Alzheimer Center Limburg has executed an awareness campaign to inform the general public that healthy behaviors, for instance related to diet and physical exercise, can improve their brain health (WeZijnZelfhetMedicijn.nl). These types of behaviors target vascular risk factors, and our findings emphasize the vascular contribution to brain health. Our findings therefore support the necessity of such a campaign, which can in turn help to communicate the general message supported by our findings to the general public, for instance through media or community participation. 



\section{Inge Verheggen}

Date of birth: 23 $3^{\text {rd }}$ of September 1991

Place of birth: Weert

Work experience

2015-2020 | PhD candidate

School for Mental Health and Neuroscience (MHeNS), Department of Psychiatry and Neuropsychology, Alzheimer Center Limburg, Maastricht University

Topic: Imaging blood-brain barrier function in aging

Education

2013-2015 |

Research Master in Cognitive and Clinical Neuroscience, specialization Neuropsychology

Faculty of Psychology and Neuroscience, Maastricht University GPA 8.43

2010-2013 | Bachelor Psychology and Neuroscience

Faculty of Psychology and Neuroscience, Maastricht University GPA 8.88 (Cum Laude)

2012-2013। Exchange program/Maastricht Research Based Learning (MaRBLe) program abroad

Stellenbosch University (South Africa)/Maastricht University

2011-2012 I Honors Program

Faculty of Psychology and Neuroscience, Maastricht University GPA 8.28 
Curriculum Vitae

2004-2010 | Pre-university secondary education, Philips van Horne Scholengemeenschap (Weert), Cum Laude

Academic appraisals

$2020 \mid$

Magna Cum Laude Merit Award Conference International Society for Magnetic Resonance in Medicine (ISMRM) 2020

Oral presentation: Permeability of the windows of the brain: Feasibility of dynamic contrast-enhanced MRI of the circumventricular organs

2014-2015 |

\section{Successful NWO Research Talent grant application}

Nederlandse Organisatie voor Wetenschappelijk Onderzoek (NWO)

[Personal Grant I.C.M.V. number 406-15-031]

Funding for a 4-year PhD trajectory

Project: Imaging the role of blood-brain barrier function in aging

Publications

Verheggen, I. C.M., de Jong, J. J., van Boxtel, M. P., Postma, A. A., Verhey, F. R., Jansen, J. F., \& Backes, W. H. (2020). Permeability of the windows of the brain: feasibility of dynamic contrast-enhanced MRI of the circumventricular organs. Fluids and Barriers of the CNS, 17(1), 1-13.

Verheggen, I. C.M., de Jong, J. J., van Boxtel, M. P., Postma, A. A., Jansen, J. F., Verhey, F. R., \& Backes, W. H. (2020). Imaging the role of blood-brain barrier disruption in normal cognitive ageing. GeroScience, 1-14.

Verheggen, I. C.M., de Jong, J. J., van Boxtel, M. P., Gronenschild, E. H., Palm, W. M., Postma, A. A., ... \& Backes, W. H. (2020). Increase in blood-brain barrier leakage in healthy, older adults. GeroScience, 42(4), 1183-1193.

Freeze, W. M., Jacobs, H. I., de Jong, J. J., Verheggen, I. C.M., Gronenschild, E. H., Palm, W. M., ... \& Backes, W. H. (2020). White matter hyperintensities mediate the 


\section{Curriculum Vitae}

association between blood-brain barrier leakage and information processing speed. Neurobiology of Aging, 85, 113-122.

Verheggen, I. C.M., Van Boxtel, M. P. J., Verhey, F. R. J., Jansen, J. F. A., \& Backes, W. H. (2018). Interaction between blood-brain barrier and glymphatic system in solute clearance. Neuroscience \& Biobehavioral Reviews, 90, 26-33. 



\section{Dankwoord}

Alle mensen die mij hebben geholpen en gesteund tijdens dit hele traject ben ik erg dankbaar, en zonder deze mensen zou het me nooit gelukt zijn tot dit punt te komen.

Allereerst ben ik de leden van de leescommissie dankbaar dat ze de tijd en moeite hebben genomen om mijn proefschrift te lezen en te beoordelen, en zo deze laatste stap mogelijk te maken.

Dit proefschrift was er nooit geweest zonder alle mensen die bereid waren deel te nemen aan het onderzoek. Ik ben al deze proefpersonen ontzettend dankbaar voor hun tijd en inzet, en hun onmisbare bijdrage aan het tot stand komen van de data.

Natuurlijk wil ik graag mijn promotieteam bedanken. Walter, zonder jouw kennis en ideeën had dit project er nooit kunnen zijn. Je kwam altijd snel met uitgebreide en nuttige feedback op al mijn stukken, ongeacht hoe druk je het had, en je hebt me regelmatig de goede richting op gestuurd. Martin, als mijn dagelijkse begeleider heb je me veel geholpen, bijvoorbeeld met de MAAS-data, maar daarnaast heb je ook altijd oog gehad voor hoe het met me ging en het was altijd fijn met je te praten. Frans, je wist telkens weer met goede vragen en verfrissende inzichten te komen.

Ook wil ik Joost de Jong bedanken. Jouw hulp met de neuroimaging data is absoluut onmisbaar geweest. Ik ben erg dankbaar voor je geduld en je harde werk bij het verwerken van de MRI-data en het programmeren en uitvoeren van de analyses van de MRI-data. Ik wil Jaap Jansen bedanken. Je bent veel bij het project betrokken geweest, en kwam regelmatig met goede ideeën en feedback. Ik wil Linda Jacobi bedanken. Ik heb vaak gebruik mogen maken van jouw aanzienlijke expertise als neuroradioloog. Of het nu om het lokaliseren van de CVOs of het inkleuren van de choroid plexus ging, je nam de tijd om het me geduldig te leren. Ik wil Roland Kersemakers bedanken voor de hulp bij het inplannen van de MRI-scans, en natuurlijk wil ik ook alle laboranten die voor mij proefpersonen hebben gescand bedanken voor jullie expertise, jullie hulp en jullie gezelschap tijdens al deze uren. Verder wil ik Joachim Wildberger als hoofd van de 
Dankwoord

afdeling Beeldvorming bedanken, voor de mogelijkheid om mijn PhD project samen met deze afdeling uit te voeren.

Graag wil ik ook al mijn collega-onderzoekers van de afdeling Beeldvorming bedanken. May, Gerald, Laura, Lisanne, Marieke, Merel, Jennifer, Elles, Paulien, jullie zijn niet alleen hele slimme mensen die altijd bereid zijn te helpen, maar ook hele sociale mensen, die altijd in zijn voor een gezellige avond en, ook al was mijn werkplek ergens anders, die me altijd onderdeel van de afdeling hebben laten voelen.

Jos Slenter en Jan Jungerius van de afdeling Beeldvorming en Nico Rozendaal, Ron Mengelers en Joost Tan van het Alzheimer Centrum wil ik bedanken voor alle software en hardware ondersteuning, en het altijd oplossen van mijn computerproblemen.

Ook mijn collega's en ex-collega's van het Alzheimer Centrum wil ik graag bedanken:

Saartje, eerst was je bereid mij een stageplek te geven, en daarna was je bereid om samen met mij de beursaanvraag in te dienen, waardoor mijn PhD project uiteindelijk mogelijk werd. Bedankt dat je me deze kans hebt gegeven.

Ed, ik heb de samenwerking met jou altijd heel fijn gevonden. Je bent behulpzaam en betrokken. Als ik een vraag had, kwam jij direct met een uitgebreid en nuttig antwoord, en als ik een probleem tegenkwam, kwam jij direct met een oplossing. Zelfs na je pensioen bleef je bereid me te helpen.

Astrid, heel erg bedankt voor je hulp met de inclusie. Als een echte kenner van de MAASstudie heb jij het eerste contact met de proefpersonen gelegd. Het zal mij niet verbazen als ik veel van mijn deelnemers aan jou te danken heb.

Whitney, terwijl je zelf nog maar net in Maastricht was en net aan je PhD was begonnen, was je toch bereid mij te begeleiden als je stagiaire. Jouw project was de voorloper van mijn project, en ik had me geen betere voorloper kunnen wensen. Je was altijd bereid me te helpen, en ik bewonder de manier waarop je iedereen moed in weet te praten.

Linda, met jou als kantoorgenootje waren de werkdagen heel gezellig! Natuurlijk waren er de nodige frustraties, maar hoe fijn is het dan als je die meteen bij iemand kwijt kan. We hebben (soms te) lange gesprekken gevoerd over het doen van een PhD, de onderzoekswereld, de politiek en slechte reality tv. Hopelijk kunnen we dit in de toekomst blijven doen, en in ieder geval onze filmavondjes in stand houden, want er zijn nog een heleboel slechte films om af te kraken. 
Nikos, you were a great co-worker, always willing to help, but also with a great sense of humor and always in for a laugh. Thanks again for letting us stay at your place for the Vierdaagse, and for finally making me Spanakopita!

Whitney, Linda, Nikos, Lisa, Roy, jullie waren geweldige kantoorgenootjes! Ik heb genoten van onze gezellige gesprekken, tripjes naar Bandito's, koffiepauzes en lunchpauzes, en ons empirisch onderzoek naar het vaststellen van het verband tussen de kleur van de verpakking en de smaak van het chocolade eitje.

Ik wil ook al mijn andere collega's van het Alzheimer Centrum bedanken. Jullie zijn een geweldige groep mensen die fantastisch werk doen. Ik heb het Alzheimer Centrum altijd een hele fijne werkplek gevonden en ik zal het zeker gaan missen.

Natuurlijk wil ik ook mijn ouders bedanken. Jullie hebben alle mijlpalen met mij mee gevierd en me altijd laten weten dat jullie trots op me zijn, maar ook tijdens de mindere momenten hebben jullie me altijd gesteund. Ik waardeer jullie steun en aanmoedigingen ontzettend, dus heel erg bedankt.

Martin en Elze, jullie ook bedankt voor jullie interesse, steun en aanmoedigingen! Jullie huwelijk en de geboorte van mijn nichtje Amee heeft voor veel mooie momenten gezorgd de afgelopen jaren.

Daarnaast wil ik mijn studievriendinnen Melloney, Iris en Vivian bedanken. Melloney, jij was net als ik bezig met een $\mathrm{PhD}$, en daardoor konden we goed onze ervaringen bij elkaar kwijt. Onze avondjes samen betekenen veel voor mij, of het nu gezellig samen lachen is of serieuze gesprekken over problemen of twijfels. Iris, jij kan goed luisteren en bent begripvol en meelevend, dat maakt je een fijne vriendin om mee te praten. En natuurlijk ben ik ook je creativiteit dankbaar, aangezien ik daar mijn mooie cover aan te danken heb. Vivian, door jouw humor en relativeringsvermogen ging ik me altijd weer beter voelen. Er is veel gebeurd de afgelopen jaren. Jullie hebben bewezen dat jullie er niet alleen in de goede, maar ook in de mindere tijden zijn. Jullie zijn er altijd voor me geweest en hebben altijd geprobeerd me de goede richting in te helpen. Ik ben jullie veel verschuldigd, en daar kan ik jullie niet genoeg voor bedanken.

Emmy, ook al konden we elkaar meestal alleen via de telefoon spreken, je bent toch altijd een belangrijk persoon voor me gebleven de afgelopen jaren, bij wie ik alles kwijt kan. Als er iets gebeurt, ben je nog steeds een van de eerste die ik het laat weten! 
Dankwoord

Saskia, Jelleke, Astrid en Nadia, bedankt voor de afleiding die de gezellige momenten met jullie hebben geboden, en de goede gesprekken!

Perkins, bedankt voor alle knuffels en het kroelen, en je onmisbare gezelschap.

Het was een hele weg, en niet altijd gemakkelijk, maar uiteindelijk zijn we er gekomen! 